\title{
Modelling plant trait variability in changing arid environments
}

\author{
Dissertation \\ to attain the doctoral degree (Dr. rer. nat)
}

of the Faculty of Forest Sciences and Forest Ecology

Georg-August-Universität Göttingen

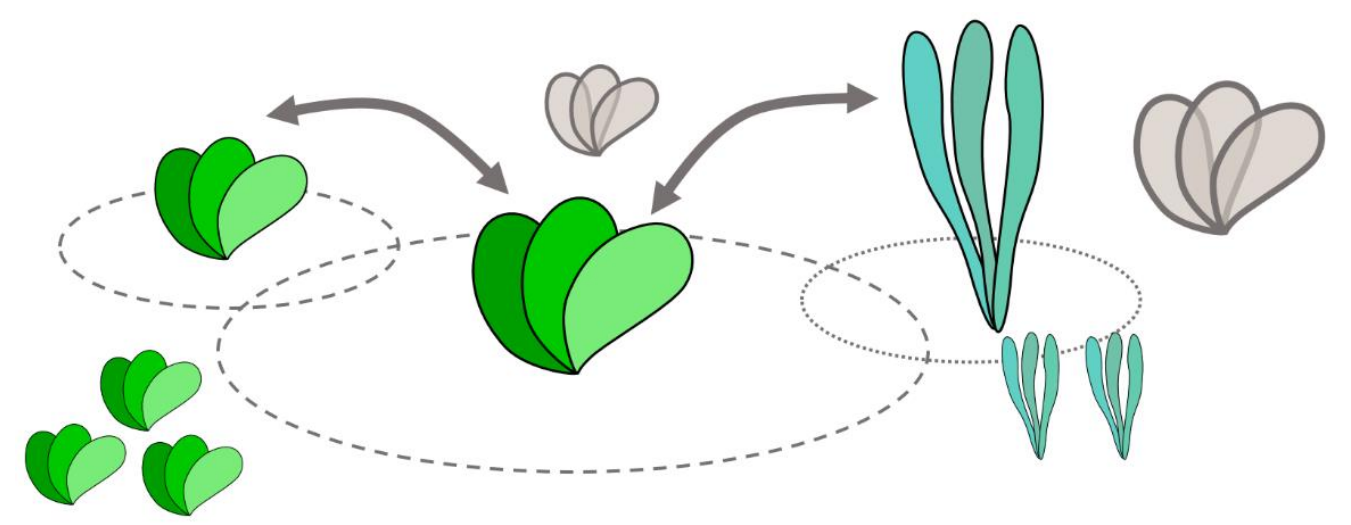

Submitted by

Liubov Zakharova

born on the 04.01.1985 in Woroschilowgrad, Ukraine

Göttingen, June 2020 
Daily supervisor: Dr. Katrin M. Meyer

1. Referee: Prof. Dr. Kerstin Wiegand

2. Referee: Prof. Dr. Holger Kreft

Date of oral examination: 14.09.2020 


\section{Table of content}

Summary

Zusammenfassung

General introduction

Chapter 1. Trait-based modelling in ecology: a review of two decades of research

Abstract

1.1. Introduction

1.2. Methods

1.3. Glossary

$\begin{array}{ll}\text { 1.4.1. Trait-based modelling of plants in terrestrial ecosystems } & 27\end{array}$

1.4.2. Trait-based modelling of animals in terrestrial ecosystems $\quad 38$

$\begin{array}{ll}\text { 1.4.3. Trait-based modelling of aquatic ecosystems } & 41\end{array}$

1.4.4. Trait-based models on microorganisms and soil decomposers $\quad 48$

1.4.5. General insights on trait-based modelling $\quad 49$

$\begin{array}{lr}\text { 1.5. Discussion } & 50\end{array}$

Acknowledgements $\quad 54$

Chapter 2. Combining trait- and individual-based modelling to understand desert plant community dy- 55 namics

$\begin{array}{ll}\text { Abstract } & 55\end{array}$

$\begin{array}{lr}\text { 2.1. Introduction } & 56\end{array}$

2.2. Methods $r$

2.2.1. Research site $\quad 59$

2.2.2. Developing a trait- and individual-based model $\quad 59$

\begin{tabular}{ll} 
2.2.3. & Model analysis \\
\hline
\end{tabular}

$\begin{array}{ll}\text { 2.3. Results } & 74\end{array}$

$\begin{array}{lll}\text { 2.3.1. } & \text { Model development and validation } & 74\end{array}$

\begin{tabular}{ll} 
2.3.2. & Results of sensitivity analysis \\
\hline
\end{tabular}

$\begin{array}{lr}\text { 2.4. Discussion } & 81\end{array}$

Acknowledgements $\quad 85$

Chapter 3. The success of plant survival strategies under spatial and temporal heterogeneity in warm de- 87 serts: a simulation experiment
Abstract

$\begin{array}{ll}\text { 3.1. Introduction } & 88\end{array}$

3.2. Methods 91

\begin{tabular}{ll} 
3.2.1. & Study area \\
\hline
\end{tabular}

3.2.2. Model parameterization and calibration $\quad 92$

$\begin{array}{ll}\text { 3.2.3. Scenarios } & 93\end{array}$

$\begin{array}{lr}\text { 3.3. Results } & 94\end{array}$

$\begin{array}{llr}\text { 3.4. Discussion } & 98\end{array}$

$\begin{array}{lr}\text { Acknowledgements } & 100\end{array}$ 
4.1. Introduction 102

4.2. Methods 105

4.2.1. Research site and materials $\quad 105$

4.2.2. Experimental design 106

$\begin{array}{lll}\text { 4.2.3. Statistical analysis } & 108\end{array}$

$\begin{array}{ll}\text { 4.2.3.1. Generalized linear models to assess average trait responses } & 108\end{array}$

4.2.3.2. n-hypervolumes of functional trait space $\quad 109$

$\begin{array}{lll}\text { 4.3. } & \text { Results } & 111\end{array}$

4.3.1. Generalized linear models to assess average trait responses $\quad 111$

$\begin{array}{ll}\text { 4.3.2. n-hypervolumes of functional trait space } & 114\end{array}$

$\begin{array}{lr}\text { 4.4. Discussion } & 118\end{array}$

$\begin{array}{ll}\text { Acknowledgements } & 122\end{array}$

Chapter 5. Metamodels for evaluating, calibrating and applying agent-based models: A review 123

$\begin{array}{ll}\text { Abstract } & 123\end{array}$

$\begin{array}{lr}\text { 5.1. Introduction } & 124\end{array}$

$\begin{array}{ll}\text { 5.2. Methods } & 125\end{array}$

$\begin{array}{llr}\text { 5.2.1. } & \text { Searching procedure } & 125\end{array}$

5.2.2. Categorization of MMs and purpose of application $\quad 125$

$\begin{array}{ll}\text { 5.2.3. Assessment of MM quality and implementation effort } & 127\end{array}$

$\begin{array}{ll}\text { 5.3. Results and Discussion } & 130\end{array}$

$\begin{array}{ll}\text { 5.4. Conclusions } & 137\end{array}$

$\begin{array}{ll}\text { Acknowledgements } & 138\end{array}$

$\begin{array}{lr}\text { General discussion and conclusions } & 139\end{array}$

$\begin{array}{ll}\text { References } & 146\end{array}$

$\begin{array}{ll}\text { Appendix } & 167\end{array}$

$\begin{array}{lr}\text { Chapter } 1 & 168\end{array}$

$\begin{array}{lr}\text { Chapter } 2 & 184\end{array}$

$\begin{array}{lr}\text { Chapter } 3 & 195\end{array}$

$\begin{array}{lr}\text { Chapter } 4 & 205\end{array}$

$\begin{array}{lr}\text { Acknowledgements } & 207\end{array}$

$\begin{array}{lr}\text { Curriculum vitae } & 209\end{array}$ 


\section{Index of figures}

Figure 1. Research sites across the Negev desert with annual plant communities dominated by Anastatica hiero-

chuntica

Figure 1.1. Conceptual overview of trait-based models compared to species-based models

Figure 1.2. Papers on trait-based models included in the final list of reviewed models

Figure 1.3. Overview of the models of plants in terrestrial ecosystems based on their main purposes and research

Figure 1.4. Trait-based modelling differs from individual-based modelling in the main entities of the models and in the way interactions are represented

Figure 2.1. Conceptual scheme of the ATID-model

Figure 2.2. Causal diagram of the processes at the core of the ATID-model

Figure 2.3. Schematic representation of the simulated topographical objects and their influence on the processes

of plant growth and seed germination

Figure 2.4. 10-year simulation of the ATID model

Figure 2.5. Results of the sensitivity analysis for number of adult plants as output metric and for the scenario with

ZOI-calculation after Lin et al., 2012 and dew present in the model

Figure 2.6. Results of the sensitivity analysis for number of seeds as output metric and for the scenario with ZOIcalculation after Lin et al., 2012 and dew present in the model

Figure 2.7. Results of the sensitivity analysis for number of adult plants for 'Anastatica' as output metric and for the scenario with ZOI-calculation after Lin et al., 2012 and dew present in the model

Figure 2.8. Results of the sensitivity analysis for biomass as output metric and for the scenario with ZOIcalculation after Weiner \& Damgaard, 2006 and dew absent in the model

Figure 2.9. Results of the sensitivity analysis for biomass as output metric and for the scenario with ZOIcalculation after Lin et al., 2012 and dew absent in the model

Figure 2.10. Results of the sensitivity analysis for number of adult plants for 'Anastatica' as output metric and dew absent in the model

Figure 3.1. Number of plant individuals of the species with the protective-competition strategy and with the escape-colonization strategy over simulated time in four scenarios

Figure 3.2. Number of seeds produced by the species with the protective-competition strategy and with the escape-colonization strategy over simulated time in four scenarios

Figure 3.3. Mean biomass of the species with the protective-competition strategy and with the escapecolonization strategy over simulated time in four scenarios

Figure 3.4. Proportion of the mean biomass of the species with the protective-competition strategy and with the escape-colonization strategy over simulated time in four scenarios

Figure 4.1. Sites of origin of the mother plants of the seeds in the experiment across the Negev Desert, Israel

Figure 4.2. Volumes of hypervolumes as proxies of trait spaces constructed for the populations under the control salinity treatment for all the sites of origin based on three, four and five traits

Figure 4.3. Volumes of hypervolumes as proxies of trait spaces constructed from three traits for the populations under the control salinity treatment vs. high salinity treatment

Figure 4.4. Volumes of hypervolumes as proxies of trait spaces constructed from four traits for the populations under the control salinity treatment vs. high salinity treatment

Figure 4.5. Volumes of hypervolumes as proxies of trait spaces constructed from five traits for the populations under the control salinity treatment vs. high salinity treatment

Figure 5.1. Number of reviewed papers and categorized MM families according to the publication year 
Figure 5.2. Results of the MM quality and implementation effort assessment for the application aim of sensitivity analysis

Figure 5.3. Results of the MM quality and implementation effort assessment for the application aim of calibration

Figure 5.4. Results of the MM quality and implementation effort assessment for the application aim of prediction

Figure 5.5. Results of the MM quality and implementation effort assessment for the application aim of upscaling

Figure 6. A conceptual scheme showing the flow of theoretical concepts and data from one chapter to another and links between all of them in order to achieve the overarching aim of the thesis

Figure A2.1. Flow-chart of the ATID-model

Figure A2.2. Results of the sensitivity analysis for six output metrics (three for each of two generic species) and for eight scenario combining ZOI-calculation, presence or absence of dew in the model and seed dispersal strategies

Figure A3.1. Results of simulation runs for the species with the protective-competition strategy and with the escape-colonization strategy over simulated time for the neutral scenario corresponding to the 'Meishar' site

Figure A3.2. Results of simulation runs for the species with the protective-competition strategy and with the escape-colonization strategy over simulated time in four scenarios 


\section{Index of tables}

Table 2.1. Potential output values of the model with the units of measurement and level of observation, which can be obtained in the model simulations

Table 3.1. The parameter values of the four simulated scenarios with respect to height difference and standard deviation of the soil surface and interannual difference in the number of days with rain

Table 4.1. Study sites and their characteristics used for the analysis

Table 4.2. Analysis of deviance table with the significance of each predictor for dry weight as a response variable

Table 4.3. Analysis of deviance table with the significance of each predictor for number of leaves as a response variable

Table 4.4. Analysis of deviance table with the significance of each predictor for seed germination probability as a response variable

Table 4.5. Analysis of deviance table with the significance of each predictor for osmolyte concentration as a response variable

Table 4.6. Analysis of deviance table with the significance of each predictor for stomatal conductance $g_{s}$ as a response variable

Table 4.7. Analysis of deviance table with the significance of each predictor for photosynthetic rate $A_{N}$ as a response variable

Table 4.8. The similarity between the trait spaces of populations from the same site of origin that were exposed to the high salinity treatment or the control treatment

Table 5.1. Complete list of all databases used for the review presented

Table 5.2. MM classification derived from the accepted papers with MM applications in an ABM context

Table 5.3. Common purposes of emulating ABMs by means of MMs

Table 5.4. Criteria applied for assessing the MM quality for the given purpose of emulating the ABM

Table 5.5. Criteria applied for assessing the MM implementation effort for the given application aims

Table 5.6. The overall MM quality and implementation effort was calculated for each application according to the mean ratings of each of the three criteria for quality (CU, NE and $\mathrm{SuA}$ ) and effort (AG, OA and RC)

Table 5.7. Calculated inter-rater reliability for the rating criteria with evaluation following Cicchetti (1994)

Table A1.1. Complete list of models included in this review

Table A1.2. Search terms and history of the literature search

Table A2.1. Model parameters for sensitivity analysis

Table A2.2. Model variables

Table A3.1. Model parameters for simulation of Meishar community

Table A4.1. Output of the summary()-function in R applied to the GLMs as described in the main text for six plant

functional traits as response variables: dry weight, number of leaves, seed germination probability, osmolyte concentration, stomatal conductance, and photo-synthetic rate

Table A4.2. The similarity between the trait spaces of the populations of Anastatica hierochuntica from different sites of origin compared pairwise 


\section{List of abbreviations}

$\mathrm{ABC}$

$\mathrm{ABM}$

AG

AI

$\mathrm{A}_{\mathrm{N}}$

ATID-model

CATs

CODE

CSR triangle

$\mathrm{CU}$

Df

DGVM

EC

g

GLM

$\mathrm{g}_{\mathrm{s}}$

HS

IBM

IPCC

$\mathrm{m}$

MM

$\mathrm{NE}$

$\mathrm{OA}$

ODD

ODE

PDE

$\mathrm{RC}$

sd

SR

$\mathrm{SuA}$

ZOI
Approximate Bayesian Computation

Agent-based model

Availability of open access guiding sources

Aridity index

Photosynthetic rate

Anastatica Trait-based and Individual-based Desert model

Community assembly via trait selection

Compartment ordinary Differential Equation

Competitors, Stress tolerators, Ruderals triangle

Consideration of uncertainty

Degrees of freedom

Dynamic Global Vegetation Models

Soil electrical conductivity

Gram

Generalized linear model

Stomatal conductance

Treatment with adding salt solution

Individual-based model

Intergovernmental Panel on Climate Change

Meter

Metamodel

Number of evaluation criteria

Out-of-the-Box Applicability

Overview, Design concepts, Details

Ordinary Differential Equation

Partial Differential Equation

$\mathrm{R}$ coverage

Standard deviation

Species richness

Suitability Assessment

Zone of influence 


\section{Summary}

Communities in arid environments are especially vulnerable to global change because they experience highly unpredictable environmental conditions. The fate of communities in an uncertain future may be elucidated by understanding the drivers of these communities. The interplay between community drivers may be unravelled by using approaches based on functional traits because traits describe plant strategies and the responses of communities to environmental changes. Furthermore, inter- and intraspecific trait variability provides the necessary cues to identify survival strategies of desert plants under fluctuating environmental conditions. However, studying desert plant communities is challenging due to the spatial and temporal heterogeneity of arid environments. Modelling approaches support and complement empirical trait-based approaches in exploring desert plant communities and their drivers and dynamics in changing arid environments.

The overarching aim of this thesis was to explore intra- and inter-specific variability of functional traits in arid environments and to investigate how this variability affects the ability of plants to tolerate aridity stress and succeed in competition with their neighbours. To address this aim, I developed, implemented and analysed a spatially explicit individual- and trait-based simulation model, conducted a simulation experiment, analysed data from model simulations and empirical experiments and synthesized the literature on trait-based models and metamodelling approaches. My research was focused on annual plant communities dominated by the True Rose of Jericho (Anastatica hierochuntica L.) in the Negev desert in Israel.

According to the review in chapter 1, trait-based models are a suitable method to predict changes in community patterns under global change and to understand the underlying mechanisms of community assembly and dynamics. Combining modelling and trait-based approaches overcomes technical challenges, scaling problems, and data scarcity. Specifically, a combination of trait-based approaches and individual-based modelling was recommended to simplify the parameterization of models and to capture plant-plant interactions at the individual level, and to explain community dynamics. 
In chapter 2 , in line with the major claim of chapter 1 , the spatially explicit trait- and individual-based ATID-model was developed, implemented and analysed to explore how community dynamics arise from plant traits and the interactions among plants and with their environment. The sensitivity analysis of the model highlighted plant functional traits as key drivers of community dynamics and indicated that environmental factors were less important in the model. The outlined traits included both those traits that are involved in plant-plant interactions, such as relative growth rate and maximum biomass, and those that promote tolerance to abiotic stress, such as dormancy and germination probability. Among the environmental factors, the most influential factors were soil water availability and precipitation. The special role of functional traits in the community dynamics of desert annual plants indicates the importance of trait-based strategies as an adaptation to the stressful arid environment.

Chapter 3 addresses the results from a simulation experiment that was conducted in the ATIDmodel. This experiment explored the influence of functional traits involved in two survival strategies defined in the study as 'protective-competition' and 'escape-colonization' strategies on community dynamics. These strategies differed not only in seed size and the number of seeds, but also in the plant functional traits related to competition and survival, which were highlighted in the sensitivity analysis of the model from chapter 2. Merging the colonization-competition trade-off with escape in time and space into one strategy set provided a more realistic representation of species because the merged strategies related to the entire plant life cycle.

To gain more understanding on empirical trait distributions, in chapter 4 data on intraspecific trait variability and trait spaces of the desert annual plant $A$. hierochutica from a nethouse experiment were analysed. High salinity had significant effects on the average values of plant functional traits. Additionally, salinity stress affected the intraspecific trait spaces differentially with respect to the environmental conditions of the site of origin. Trait spaces of the populations originating from the same site but exposed to different salt stress levels became more dissimilar with increasing environmental aridity. Thus, intraspecific trait variability and salinity effects turned out to be essential in revealing population- and community-level processes in deserts and should be considered in future versions of the ATID-model. 
In support of the future development of the ATID-model developed in chapter 2, common metamodel types and the purposes of their usage for individual-based models were reviewed and evaluated in chapter 5 . The review considered 40 metamodels applied for sensitivity analysis, calibration, prediction and scaling-up of individual-based models and can be used as a guide for the implementation and validation of metamodels.

Overall, this thesis, and particularly the ATID-model analyses, highlights how trait-based modelling approaches can contribute to understanding the interplay between key drivers of desert plant communities in arid environments. The accompanying analysis of the nethouse experiment and critical literature reviews outline future extensions of the model and the ways to overcome the technical challenges and data scarcity identified in this thesis. Moreover, this thesis advocates for more intensive studies of the strategies of desert annual plants to survive in temporally and spatially heterogeneous environments with a focus on plant functional traits. Thus, the modelling framework presented in this thesis provides the basis for future research on the fate of communities in arid environments under global change. 


\section{Zusammenfassung}

\section{Modellierung der Variabilität von Pflanzen-Traits auf Populations- und Lebensgemeinschafts- ebene in ariden Gebieten mit Umweltveränderungen}

Lebensgemeinschaften in ariden Gebieten sind angesichts globaler Umweltveränderungen besonders anfällig, da sie höchst unvorhersagbaren Umweltbedingungen ausgesetzt sind. Das Schicksal von Gemeinschaften in einer ungewissen Zukunft kann durch das Verständnis der Triebkräfte dieser Gemeinschaften aufgeklärt werden. Das Zusammenspiel der Triebkräfte der Gemeinschaften kann mit Hilfe von Ansätzen entschlüsselt werden, die auf funktionalen Merkmalen (Traits) basieren, weil sie Pflanzenstrategien und die Reaktionen der Gemeinschaften auf Umweltveränderungen beschreiben können. Darüber hinaus liefert die inter- und intraspezifische Variabilität der Traits die notwendigen Anhaltspunkte für die Identifizierung von Überlebensstrategien von Wüstenpflanzen unter wechselhaften Umweltbedingungen. Die Erforschung von Wüstenpflanzengemeinschaften könnte jedoch aufgrund der räumlichen und zeitlichen Heterogenität der ariden Umweltbedingungen eine Herausforderung darstellen. Modellierungsansätze unterstützen und ergänzen empirische, trait-basierte Ansätze bei der Erforschung von Wüstenpflanzengemeinschaften und ihrer Triebkräfte und Dynamik in sich verändernden ariden Gebieten.

Das Gesamtziel dieser Arbeit war es, die intra- und interspezifische Variabilität der funktionalen Traits in ariden Umgebungen zu erforschen und zu untersuchen, wie sich diese Variabilität auf die Fähigkeit von Pflanzen auswirkt, Trockenstress zu tolerieren und in der Konkurrenz mit ihren Nachbarn erfolgreich zu sein. Um dieses Ziel zu erreichen, habe ich ein räumlich-explizites individuen- und trait-basiertes Simulationsmodell entwickelt, implementiert und analysiert, ein Simulationsexperiment durchgeführt, Daten aus empirischen Experimenten analysiert und einen Überblick der Literatur zu trait-basierten Modellen und Metamodellierungsansätzen zusammengestellt. Meine Forschung basiert auf Daten zu annuellen Pflanzengemeinschaften in der Wüste Negev in Israel, die von der Echte Rose von Jericho (Anastatica hierochuntica) dominiert werden. 
Die Literaturzusammenschau in Kapitel 1 offenbart, dass trait-basierte Modelle eine geeignete Methode sind, um Veränderungen in den Mustern von Gemeinschaften unter globalen Veränderungen vorherzusagen und die zugrunde liegenden Mechanismen der Zusammensetzung und Dynamik von Lebensgemeinschaften zu verstehen. Durch die Kombination von Modellierung und trait-basierten Ansätzen lassen sich technische Herausforderungen, Skalierungsprobleme und Datenknappheit überwinden. Insbesondere wurde eine Kombination aus trait-basierten Ansätzen und individuenbasierter Modellierung empfohlen, um die Parametrisierung der Modelle zu vereinfachen, Interaktionen zwischen Pflanzen auf individueller Ebene zu erfassen und die Gemeinschaftsdynamik zu erklären.

Eine Forderung aus Kapitel 1 umsetzend wurde in Kapitel 2 das räumlich-explizite, trait- und individuenbasierte ATID-Modell entwickelt, implementiert und analysiert, um zu untersuchen, wie Gemeinschaftsdynamiken aus Pflanzentraits und Interaktionen von Pflanzen untereinander und mit ihrer Umwelt entstehen. Die Sensitivitätsanalyse des Modells hob die funktionalen Traits von Pflanzen als Schlüsselfaktoren der Gemeinschaftsdynamik hervor, wobei den Umweltfaktoren im Modell eine relativ geringere Bedeutung zugewiesen wurde. Die sensitivitätverursachenden Traits umfassten sowohl solche Traits, die an den Pflanze-Pflanze-Interaktionen beteiligt waren, wie zum Beispiel die relative Wachstumsrate und maximale Biomasse, als auch solche, die die Toleranz gegenüber abiotischem Stress fördern, wie die Keimruhe und Keimungswahrscheinlichkeit. Unter den Umweltfaktoren waren die Verfügbarkeit von Bodenwasser und Niederschlag die einflussreichsten Faktoren. Die besondere Rolle von funktionalen Traits in der Gemeinschaftsdynamik einjähriger Wüstenpflanzen zeigt die Bedeutung trait-basierter Strategien als Anpassung an die harschen Bedingungen in ariden Gebieten.

Kapitel 3 befasst sich mit den Ergebnissen eines Simulationsexperiments, das mit dem ATIDModell durchgeführt wurde. Dieses Experiment untersuchte den Einfluss funktionaler Traits auf die Gemeinschaftsdynamik, die bei zwei Überlebensstrategien eine Rolle spielen, die in der Studie in einem neuen Strategiekonzept als "Schutz-Konkurrenz"- und "Flucht-Kolonisierungs"-Strategien definiert wurden. Diese Strategien unterschieden sich nicht nur in der Samengröße und der Anzahl der Samen, sondern auch in bestimmten Pflanzentraits, die mit Konkurrenz und Überleben zusammenhän- 
gen und die in der Sensitivitätsanalyse des Modells aus Kapitel 2 hervorgehoben worden waren. Die Integration der Konzepte des Kolonisierung-Konkurrenz-Trade-offs und des Entkommens in Zeit und Raum in einem neuen Strategiekonzept ergab eine realistischere Darstellung der Arten, da die integrierten Strategien den gesamten Lebenszyklus der Pflanze berücksichtigen.

Um ein besseres Verständnis empirischer Trait-Verteilungen zu erlangen, wurden in Kapitel 4 Daten zur intraspezifischen Traitvariabilität und zu Trait-Räumen der annuellen Wüstenpflanze $A$. hierochutica aus einem Gewächshausversuch analysiert. Hohe Salzkonzentrationen hatten signifikante Auswirkungen auf die Durchschnittswerte der funktionalen Traits der Pflanzen. Zusätzlich beeinflusste Salzstress die intraspezifischen Trait-Räume unterschiedlich in Bezug auf die Umweltbedingungen des Ursprungsortes der Pflanzen. Die Trait-Räume der Populationen, die vom gleichen Standort stammten, aber unterschiedlichen Salzstress-Niveaus ausgesetzt waren, wurden mit zunehmender Aridität unähnlicher. Daher erwiesen sich die intraspezifische Trait-Variabilität und die Salzeffekte als wesentlich für die Aufdeckung von Prozessen auf Populations- und Lebensgemeinschaftsebene in Wüsten und sollten in zukünftigen Versionen des ATID-Modells berücksichtigt werden.

Zur Unterstützung der zukünftigen Entwicklung des in Kapitel 2 entwickelten ATID-Modells wurden in Kapitel 5 Metamodelltypen und ihre Anwendungsbereiche in der individuenbasierten Modellierung überprüft und bewertet. Die Überprüfung berücksichtigte 40 Metamodelle, die für die Sensitivitätsanalyse, Kalibrierung, Vorhersage und Skalierung von individuenbasierten Modellen eingesetzt werden können und als Leitfaden für die Implementierung und Validierung von Metamodellen dienen können.

Insgesamt beleuchtet diese Arbeit und insbesondere die Analysen des ATID-Modells, wie trait-basierte Modellierungsansätze zum Verständnis des Zusammenspiels der Schlüsseltriebkräfte von Wüstenpflanzengemeinschaften in ariden Umgebungen beitragen können. Die begleitende Analyse des Gewächshausexperiments und die kritischen Literaturübersichten dienen als Grundlage für zukünftige Erweiterungen des Modells und die in dieser Arbeit identifizierten Wege zur Überwindung technischer Herausforderungen und Datenknappheit. Darüber hinaus empfiehlt diese Dissertation eine intensivere Untersuchung der Strategien annueller Wüstenpflanzen für das Überleben unter zeitlich 
und räumlich heterogenen Umweltbedingungen mit besonderem Schwerpunkt auf funktionalen Pflanzen-Traits. Somit bietet das in dieser Arbeit vorgestellte Grundmodell die Basis für zukünftige Forschungen über das Schicksal von Lebensgemeinschaften in ariden Gebieten unter dem Einfluss globaler Umweltveränderungen. 


\section{General introduction}

Arid environments experience changes at different levels. Besides the global climate changes that affect most of the environments and their inhabitants across the globe (IPCC, 2018), the organisms in arid environments are exposed to spatiotemporal heterogeneity in limiting environmental factors (Chesson et al., 2004; Loik et al., 2004). This heterogeneous character of the environment largely determines the existence of arid communities, causing the development of a variety of strategies by living organisms to cope with the unpredictable environmental conditions (Chesson, 2000). Plants are sessile organisms and thus highly dependent on their environment, as the adults cannot easily escape an environment that has suddenly become unfavourable (Venable and Lawlor, 1980). Desert plant strategies have contributed to the survival of arid communities in the past and, thus, might play an important role in their survival in face of global changes (Salguero-Gómez et al., 2012).

One of the ways to describe plant strategies and their functions in a community is through trait-based approaches (Westoby et al., 2002; Wright et al., 2004; McGill et al., 2006; Violle et al., 2007). In this case, each strategy would be translated to a set of functional traits that can be involved in trade-offs (Messier et al., 2017). Trait-based approaches link organismal, morphological and physiological characteristics to functions such as growth, reproduction and survival (Violle et al., 2007). Thus, by observing, measuring and analysing functional traits, conclusions can be drawn on what kind of strategy an organism applies to succeed in a given environment (Reich et al., 2003; Clark et al., 2012). However, the majority of research in functional ecology is focused on species-specific functional traits (McGill et al., 2006; Suding and Goldstein, 2008; Albert et al., 2010) or grouping species into functional types (McIntyre et al., 1995; Lavorel et al., 1997; Pausas, 1999) based on shared sets of traits. Hence, intraspecific trait variability has long been largely ignored (Bolnick et al., 2011; Violle et al., 2012) although it underlies the plasticity of species (Turcotte and Levine, 2016) and might explain their ability to adapt to changing environments (Lepš et al., 2011; Jung et al., 2014) (see also chapter 4). In some cases, for example, in harsh environments, the importance and amplitude of intraspecific trait variability can be even larger than of interspecific trait variability (Read et al., 2017). 
Thus, trait variability at different levels might be a key to understanding the survival and prospering of plant communities in changing arid environments (Albert et al., 2017; Des Roches et al., 2018).

The mechanisms underlying the survival of plants in arid environments remain understudied. One of the reasons for this gap in knowledge lies in the challenge to capture long-term community processes under heterogeneous environmental conditions in the framework of short-term empirical studies (Herben and Wildová, 2012; Chamberlain et al., 2014). Accompanying empirical studies with modelling approaches allows researchers to overcome spatial and temporal constraints and investigate the fate of simulated communities over decades. Moreover, modelling in ecology can extend and deepen empirical studies by contributing to the understanding of the underlying community mechanisms and to the development of new ecological theory (van der Putten et al., 2009; Meyer et al., 2009). Additionally, modelling tools promise lower risk of damage to natural plant communities and often lower resource requirements. Thus, supporting empirical studies of arid communities with modelling approaches appears to be a promising avenue for current and future investigation. Although a number of models have been created that are devoted to different aspects of arid environments (Howes and Abrahams, 2003; Reynolds et al., 2004; Li et al., 2018), surprisingly, only few models explicitly consider arid vegetation (Venable and Lawlor, 1980; King and Roughgarden, 1982; Chen and Reynolds, 1997; James et al., 2005; Gerlein-Safdi et al., 2018; Wang et al., 2018) or specifically plant communities (McAuliffe, 1988). Plant community models mainly exist as a special case of global models (Reick et al., 2013). The simulation model ATID developed in the course of this thesis contributes to filling this gap (see also chapters 2 and 3).

Combining trait-based approaches with tools of ecological modelling (Garrard et al., 2013; Weiss et al., 2014) seems to be an appropriate way to study plant communities in arid environments (see also chapters 1, 2 and 3). Trait-based approaches describe interactions between organisms in terms of traits, and the inherent link of traits to organismal functions provides an insight into mechanisms of population and community dynamics (Shipley et al., 2006; Lamarque et al., 2014). Traits can represent both a response to environmental changes and an effect on community properties (Lavorel et al., 1997b; Lavorel and Garnier, 2002). This is reflected in the separation into response traits and ef- 
fect traits. Incorporated into a modelling framework, effect traits serve as an input to the model and provide insights how different combinations of traits form plant strategies and community properties. Furthermore, response traits can be an output of a model reflecting how environmental changes shape the community (chapter 1). The integration of plant functional traits with modelling approaches is intuitive in the form of individual-based models (Grimm and Railsback, 2005), because individuals are the carriers of traits. Individual-based models capture ecological processes via individual interactions with each other and the environment, which leads to the emergence of population- and communitylevel dynamics (DeAngelis and Grimm, 2014). Each individual in an individual-based model is associated with a set of traits, some of which can be linked to an organismal function, i.e. be functional traits (Violle et al., 2007). Moreover, individual-based models are usually well equipped to simulate spatial and temporal heterogeneity of environments. A few studies have successfully incorporated this combined approach to pursue goals such as exploring plant growth and population dynamics (Bown et al., 2007; Taubert et al., 2012), plant-plant interactions and invasions (Radny and Meyer, 2018) as well as community assembly (Pachepsky et al., 2007) and dynamics (May et al., 2009; Seifan et al., 2012; Weiss et al., 2014) (see also chapter 1).

However, elaborated individual- and trait-based models also experience some limitations. The main limitations are requirements of high computing power for calculations and simulations and empirical data for their parameterization and calibration. These limitations of ecological modelling can be tackled with the support of adjacent disciplines. The demand for computing power of procedures such as sensitivity analysis, calibration and scaling-up can be mitigated with the implementation of metamodels (Kleijnen and Sargent, 2000; Mertens et al., 2017) (see also chapter 5). Lack of empirical data can be overcome by coupling modelling efforts with corresponding data collections and empirical experiments (van der Putten et al., 2009; Herben and Wildová, 2012). In this way, more precise information on the links between functional traits and their environment (McGill et al., 2006; Webb et al., 2010) can be obtained as well as on the characteristic trait distributions and the size of trait spaces (Mason et al., 2005) of the modelled species or communities (see also chapter 4). 
The overarching aim of my thesis was two-fold: 1) to explore intra- and inter-specific variability in functional traits in arid environments and 2) to investigate how trait variability affects the response of plants to competitive pressure and abiotic stress. To tackle this complex aim, I combined different methods such as a literature review on trait-based modelling (chapter 1) and one on metamodeling approaches (chapter 5), the development and analysis of a spatially explicit individual- and trait-based simulation model (chapters 2 and 3), and the analysis of data from empirical experiments (chapter 4). My thesis contributes to the empirical-modelling cooperation project "Population- and community-level plant trait variability in changing arid environments".

For purposes of strategic modelling and model parameterization and calibration, I used data on functional traits and environmental factors from the Negev desert in Israel. This warm desert is characterised by high aridity with a mean annual precipitation below $80 \mathrm{~mm}$ at the study sites (Berkowicz et al., 1995). In spite of these unfavourable conditions, the annual species Anastatica hierochuntica L. (Brassicaceae), the True Rose of Jericho, is widely spread in the Negev desert and dominant in some of its plant communities (Gutterman, 1994). The success of the species may partly be due to its 'protective' strategy of seed dispersal that keeps most of the seeds on the plant over a long time and disperses them in small portions when the conditions are favourable (Friedman and Stein, 1980). Field observations conducted in different parts of the Negev desert (Fig. 1) demonstrate that A. hierochunti$c a$ establishes its populations under environmental conditions that greatly differ in their precipitation, aridity, salinity and topography (Hegazy and Kabiel, 2007; Eshel et al., 2017). Moreover, A. hierochuntica grows in species-rich communities and mono-specific communities, demonstrating at the same time high plasticity in size and phenology. Therefore, communities dominated by A. hierochuntica were chosen as a target for this research. 

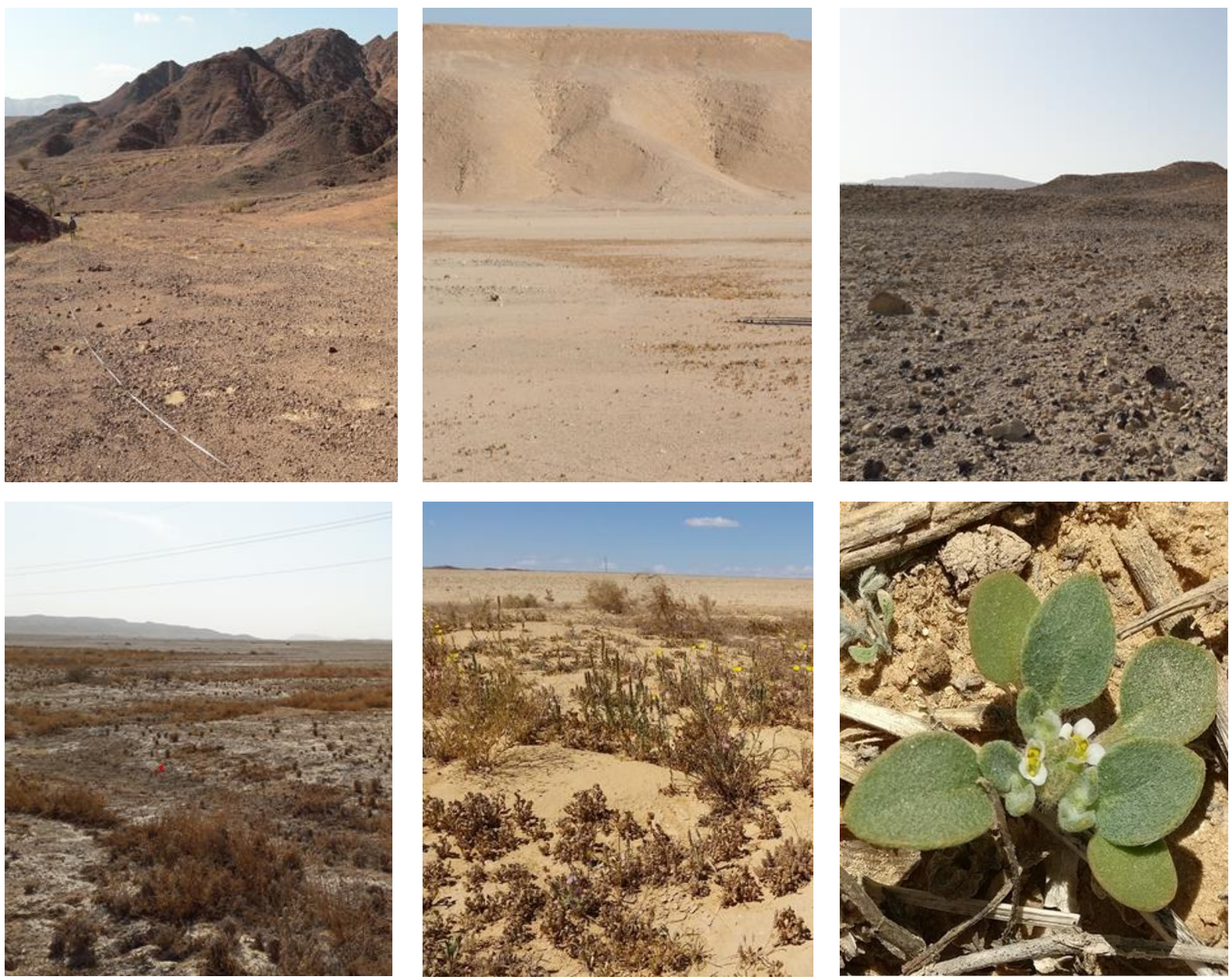

Figure 1. Research sites (from top left to bottom centre: Timna, Shaharut, Shefech Zohar, Meishar and Uvda) across the Negev desert with annual plant communities dominated by Anastatica hierochuntica (bottom right). Pictures were provided by the research group of Prof. Merav Seifan.

To achieve the overarching aim of my thesis I conducted five interconnected research projects with my co-authors. The results of these projects were prepared, submitted or already published as manuscripts and represent the five main chapters of my thesis.

\section{Chapter 1. Trait-based modelling in ecology: a review of two decades of research.}

This chapter is published in the journal "Ecological Modelling" and presents applications of trait-based models in ecology in the form of a systematic review. Special attention was paid to definitions and terminology of trait-based approaches and opportunities for cross-discipline exchange of the corresponding methods. This chapter also highlighted the advantages of combining the trait- and individual-based approach for plant community studies. This led to the development of the trait- and individual-based simulation model ATID, which is described in chapter 2. 
Chapter 2. Combining trait- and individual-based modelling to understand desert plant community dynamics.

This chapter is published in the journal "Ecological Modelling". The main goal of this research was to develop a spatially explicit two-species trait- and individual-based simulation model. The aim of the model was to identify the plant traits and environmental factors that drive the dynamics of a community of desert annuals. The chapter contains a detailed model description, a proof-ofprinciple simulation, and a comprehensive sensitivity analysis. The proof-of-principle simulation was extended into a systematic design and analysis of simulation experiments in chapter 3.

Chapter 3. The success of plant survival strategies under spatial and temporal heterogeneity in warm deserts: a simulation experiment

This chapter presents scenario analyses of the model developed in chapter 2 to explore and compare two survival strategies of annual plants in deserts under four simulated environmental scenarios. Model scenarios differed in precipitation regimes and topographical characteristics, yielding four scenarios with all combinations of high and low spatial and temporal heterogeneity. The scenario analysis provided insights into the success of plant survival strategies in a two-species community. These strategies were defined by combining the competition-colonization trade-off (Levins and Culver, 1971; Tilman, 1994) and escape-in-time versus escape-in-space seed strategies (Venable and Lawlor, 1980).

\section{Chapter 4. Intra-specific trait variability in desert annual plant communities}

In this chapter, intraspecific trait variability, trait spaces and their responses to changes in salinity in dependence on the environment of origin were investigated based on data obtained from a nethouse experiment with $A$. hierochuntica. The nethouse experiment was mainly conducted by $\mathrm{M}$. F.Arroyave Martinez at Ben-Gurion University of the Negev. This data analysis contributes to future extensions of the model developed in chapter 2 by filling the information gaps on the distribution of functional traits values at the population level and effects of high salinity on average trait values and trait spaces.

Chapter 5. Metamodels for evaluating, calibrating and applying agent-based models: A review 
This chapter covers the results of a literature review and expert assessment on metamodelling approaches. It was compiled in cooperation with a group of young scientists applying their expertise on ecological modelling. The review was led by B. Pietzsch and published in the "Journal of Artificial Societies and Social Simulation". As stressed in chapter 1 and chapter 2, individual-based models often have to compromise between the level of detail and computing power limitations. Metamodels can assist in operating individual-based models to achieve such goals as prediction, calibration, sensitivity analysis and scaling-up with relatively small computing power requirements. This review facilitates the choice of a suitable metamodel depending on the specific modelling task and thereby contributes to improving future ecological modelling. 


\title{
Chapter 1. Trait-based modelling in ecology: a review of two decades of research
}

This chapter was published as: Zakharova, L., Meyer, K. M., \& Seifan, M. (2019). Trait-based modelling in ecology: A review of two decades of research. Ecological Modelling, 407, 108703. https://doi.org/10.1016/j.ecolmodel.2019.05.008.

Authors' contribution: K. M. M., M. S. and L.Z. conceived of the idea presented. L. Z. led the writing of the manuscript with extensive input from K. M. M. and M. S..

\begin{abstract}
Trait-based approaches are an alternative to species-based approaches for functionally linking individual organisms with community structure and dynamics. In the trait-based approach, rather than focusing on the species identity of the organism, the focus is on the organism traits, which represent their physiological, morphological, or life-history characteristics. Although used in ecological research for several decades, this approach only emerged in ecological modelling about twenty years ago. We review this rise of trait-based models and trace the occasional transfer of trait-based modelling concepts between terrestrial plant ecology, animal and microbial ecology, and aquatic ecology, discuss terminology of trait-based approaches and evaluate future implementation of trait-based models, including cross-discipline exchange. Trait-based models have a variety of purposes, such as predicting changes in community patterns under climate and land-use change, understand underlying mechanisms for community assemblies, planning and assessing conservation management, or studying invasion processes. In modelling, trait-based approaches can reduce technical challenges such as computational limitations, scaling problems, and data scarcity. However, we note inconsistencies in the current usage of terms in trait-based approaches and these inconsistencies must be resolved if traitbased concepts are to be easily exchanged between disciplines. Specifically, future trait-based models may further benefit from incorporating intraspecific trait variability and addressing more complex
\end{abstract}


species interactions. We also recommend expanding the combination of trait-based approaches with individual-based modelling to simplify the parameterization of models, to capture plant-plant interactions at the individual level, and to explain community dynamics under global change.

\subsection{Introduction}

Understanding community structure and dynamics is a key element of modern ecology, especially in the light of global change (Harte and Shaw, 1995; Knapp, 2002). This understanding was traditionally mediated by species-based approaches. More recently, such approaches were complemented by approaches based on traits. Trait-based approaches are popular, because they allow the direct connection of organism performance to its functions and to the functions of higher levels of organization such as populations, communities and ecosystems. While trait-based approaches have been introduced some decades ago (Grime, 1977) and are now firmly established in empirical research (e.g. Violle et al., 2007; Suding and Goldstein, 2008), they were only introduced to modelling about twenty years ago. Given that modelling is important for understanding community structure and dynamics, trait-based modelling can reduce some of the challenges faced by species-based modelling. For example, species-based models are usually complex, difficult to parameterize and often produce outcomes that cannot be generalized to other species. Trait-based models often require less parameterization effort than species-based models, facilitate scaling-up, and produce more generalizable results that can be projected to other systems and be used to fill gaps in species knowledge. Trait-based modelling reinforces simplification, which is at the core of all modelling, because it focuses on simplified community structure, based on the organismic functions. The drawback of such simplification is that the results of trait-based models may not always be very well comparable with corresponding species-based modelling results. Here, we review the rise of traitbased models over the past twenty years, highlighting their main fields of application and pointing out avenues for future trait-based modelling. 
Traits arose from the concept of plant functional groups and these groups were the first published classification of organisms according to function (based on morphology and physiology) instead of taxonomy (Raunkiaer, 1934; Grime, 1974). The next wave of interest into functional groups was led by the desire to predict community and ecosystem responses to environmental change (Diaz and Cabido, 1997; Lavorel et al., 1997; Chapin et al., 2000). Grime's (1977) CSR triangle was the first globally accepted concept propagating continuous functional traits in contrast to discrete functional groups such as herbs, shrubs and trees. However, the focus of functional ecology shifted only much later from functional groups to functional traits and thus from species grouped because they use similar strategies to the similar characteristics underlying those strategies (Yang et al., 2015b). Distinct aspects of strategies were reflected in sets of correlated traits that were defined as trait dimensions (Westoby et al., 2002). This shift from a species-based approach to a trait-based approach is described as the 'Holy Grail of Ecology' (Lavorel and Garnier, 2002). This approach involves the use of plant functional traits, rather than species identities, to generalize complex community dynamics and to predict the effects of environmental changes (Suding and Goldstein, 2008).

Functional traits not only help derive individual strategies (Westoby, 1998; Wright et al., 2004), but also to connect them to functions at organizational levels higher than those of the species such as the community or ecosystem level. There are four requirements for a trait (Lavorel et al. 2007): It should be connected with a function; It should be relatively easy to observe and quantify; It should be possible to measure it in a standardized way across a wide range of species and environmental settings; And it should have a range of values that is comparable among individuals, species and habitats. Trait-based ecology is further based on the assumption that trade-offs and constraints have shaped phenotypic variation in different trait dimensions (Grime, 1977; Westoby, 1998).

Sets of plant traits that reliably represent the processes of growth, survival, and reproduction (Violle et al., 2007) make it possible to facilitate and generalize empirical and modelling studies. Therefore, researchers attempted to define a universal set of traits. Pachepsky et al. (2001) identified twelve critical traits that affected resource uptake, the area over which resource is captured, the internal allocation of resources between structure, storage and reproduction, time of reproduction, number of progeny produced, dispersal of progeny, and survival. Other researchers used smaller 
numbers of traits. The leaf economics spectrum, for example, contains only six traits (Wright et al., 2004). Díaz et al. (2015) also used six traits but not those of the leaf economics spectrum, and several researchers even used a set with as few as three traits (Westoby, 1998; Westoby et al., 2002; Wright et al., 2004; Chave et al., 2009; Garnier and Navas, 2012). Thus, rather than applying a universal trait set, modern use of the concept implies a selection of a small set of critical functional traits specific to the needs of a specific study and dependent on the specific organisms for which strategies are being described.

Using trait-based approaches overcomes some of the well-known problems of species-based approaches. In trait-based approaches, for example, it is possible to directly connect community functions such as production to environmental changes via functional traits. Moreover, the trait-based approach is an intuitive approach for addressing evolutionary processes because evolution selects organisms in a community according to their function and not their taxonomy. Trait-based approaches are, furthermore, more suitable than species-based approaches for generalizations across species as they are not tied to taxonomy. In addition, trait-based approaches benefit from the rapid expansion of trait databases more than species-based approaches, because trait-based approaches are not dependent on species-specific trait information; particularly trait-based models can either fill information gaps with trait data from species related to a target species or not use species at all and only work with trait value distributions. Trait databases are especially well developed for plants (Kleyer et al., 2008; Kattge et al., 2011).

Although current trait-based approaches have several benefits, they also have some shortcomings not present in species-based approaches. One of these is the choice of appropriate functional traits and their trade-offs with other traits given that a great diversity of traits are available (Funk et al., 2017). Furthermore, traits differ intraspecifically but these differences are often neglected (Violle et al., 2012; Bolnick et al., 2011). Existing trait databases are usually of limited use when it comes to species interactions, intraspecific trait variation and variable environmental settings (Funk et al., 2017). In addition, the theoretical assumptions of trait-based studies are not always supported by experimental data (Suding and Goldstein, 2008). These shortcomings can be overcome by closer 
cooperation between empirical and theoretical researchers and by the development of standards for trait data collection (e.g. Garnier and Shipley, 2001; Pérez-Harguindeguy et al., 2013).

In the most recent 20 years trait-based approaches have entered ecological modelling. The main advantage of modelling over empirical approaches is that it allows the comparison of several scenarios with different sets of assumptions, so conducting virtual experiments. This makes possible the systematic exploration of the outcomes under each set of assumptions and the elucidation of the mechanisms underlying the patterns observed. Using models therefore avoids the costs and risks of real-world experiments. Trait-based models may contain species as carriers of traits, but they also work without explicitly modelling species. In species-based models, interactions occur at the level of species (potentially depending on species traits), whereas in trait-based models, it is usually the traits that are subject to effects and responses (potentially depending on trade-offs; Fig. 1.1). Importantly for this distinction, models based on discrete functional types or functional groups are not part of our definition of trait-based models, which requires continuous trait values (although we mention some examples in sections 1.4.1.5.Trait-based dynamic global vegetation models (DGVMs), 1.4.2.Traitbased modelling of animals in terrestrial ecosystems and 1.4.4.Trait-based models on microorganisms and soil decomposers). In principal, trait-based models consist of combinations of functional traits that respond to environmental changes (response traits) and affect community and ecosystem properties (effect traits) (Fig. 1.1). Trait-based models should also account for the shape of the distribution of these traits, which often has to be derived from empirical observations (e.g. (Gaedke and Klauschies, 2017). Implementing trait-based approaches for modelling may also help overcome the high data demand of species-based models (Garrard et al., 2013; Weiss et al., 2014), simply due to the fact that traits usually represent more than one species. For the same reason, trait-based modelling may also reduce computing times. Moreover, using traits in modelling can facilitate scaling of physiological processes to global scales (Shipley, Vile, \& Garnier, 2006; Lamarque et al., 2014), because traits can function as a common currency across scales in these models. 


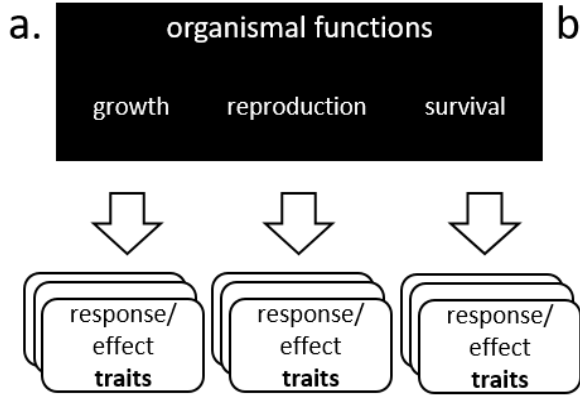

Functional traits as performance indicators

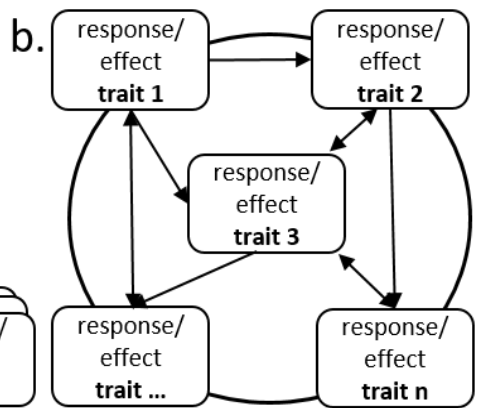

Community assembly in trait-based models

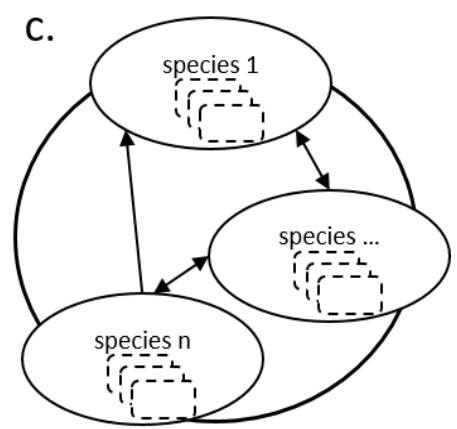

Community assembly in species-based models

d.
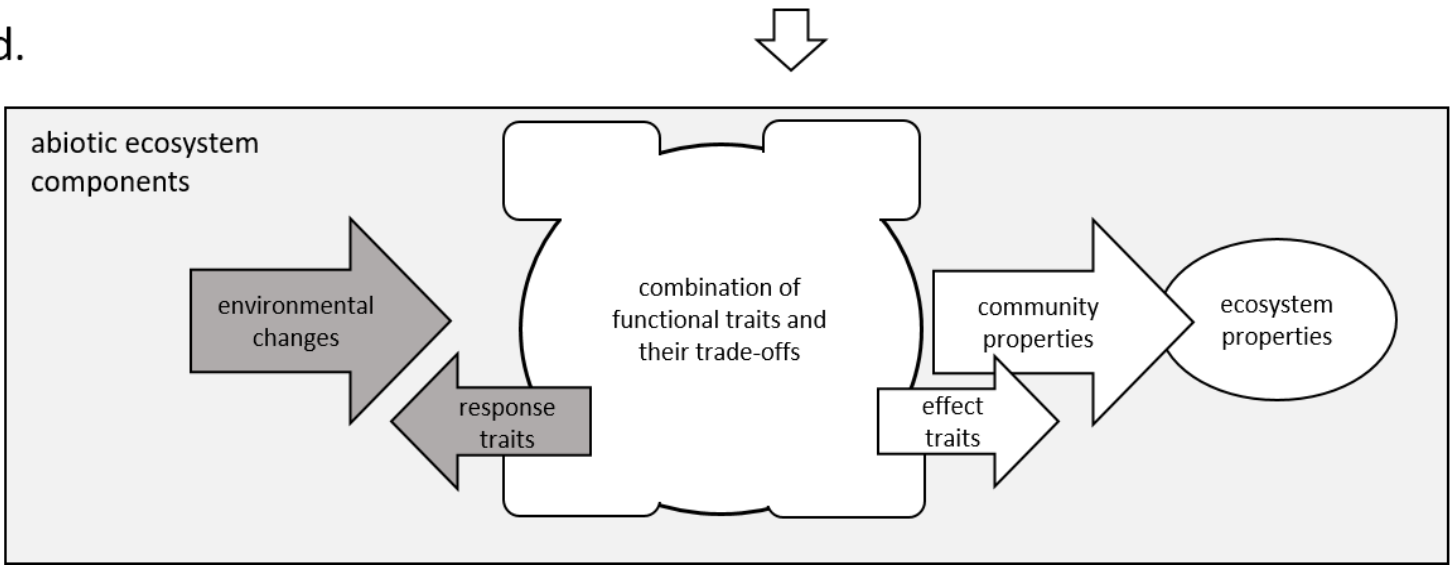

Figure 1.1. Conceptual overview of trait-based models compared to species-based models. Functional response and effect traits (rounded rectangles) are performance indicators that are related to organismal functions (a). Trait-based models represent community (circle) assembly by interacting functional response and effect traits, which may be connected via tradeoffs (b). Species-based models represent community assembly by interacting species that may implicitly contain traits (c). Trait-based models can be divided into models that use functional traits only as static inputs (white elements in d) that affect community and ecosystem properties and models that use functional traits both as inputs and dynamic outputs (white and grey elements in d). Response traits change dynamically depending on changing environmental conditions.

Given the advantages of trait-based approaches, it is still surprising that their incorporation into the tool-kit of ecological modelling has been slow and that they are applied in proportionally fewer cases of modelling than of empirical work. In this paper, our aim is to systematically review applications of trait-based models in ecology. More specifically, we 1) discuss definitions and terminology of trait-based approaches, 2) evaluate how trait-based models are used in different disciplines, and 3) identify avenues for the future implementation of trait-based models, including cross-discipline exchange. The trait-based modelling applications in this review contribute to identifying principles that underlie spatiotemporal community dynamics, exploring species distributions, investigating species interactions, scaling ecosystem processes from individual traits to 
ecosystem functioning, explaining the consequences of climate and land-use changes for community dynamics, and also supporting conservation and invasion studies.

\subsection{Methods}

This paper is based on a systematic literature review. We searched for papers using a topic search on the "Web of Science Core collection". We first used the search term "trait-base*" AND model* and in a second search "traitbase*" AND model*. The first search yielded 772 papers all of which turned out to postdate 1978. The second search added 4 papers, which were from the period 2010-2018. We excluded all papers from obviously irrelevant fields, such as psychology, medicine, engineering, business, management, history, industrial relations, linguistics, education, nutrition, and biotechnology (Appendix. Tab. A1.2). After this filtering of both searches, we retained 623 papers that focused on ecology and related biological sciences. These ecological and biological publications were the most recent among all the papers we found. In addition to the publications found during this systematic literature search, we also included papers discovered by the snowball principle, i.e. papers cited in papers already selected. We also included additional publications recommended by experts in the field. We finalized our research by selecting only those papers from our compilation that directly addressed concrete trait-based models. We excluded pure genetics, toxicology, climate and evolution studies, because we wanted to focus on ecological studies. We did not consider studies that focused on statistical analysis of empirical data, but we did include statistical models if the focus was on the model such as in species distribution modelling. Our focus was on primary modelling papers, so that we only referred to secondary modelling papers that discuss, use, extend or review previously published models when they introduce a new trait-based perspective. We did not consider editorial material or technical software descriptions. This procedure yielded 188 papers (Appendix. Tab. A1.1, Fig. 1.2). 


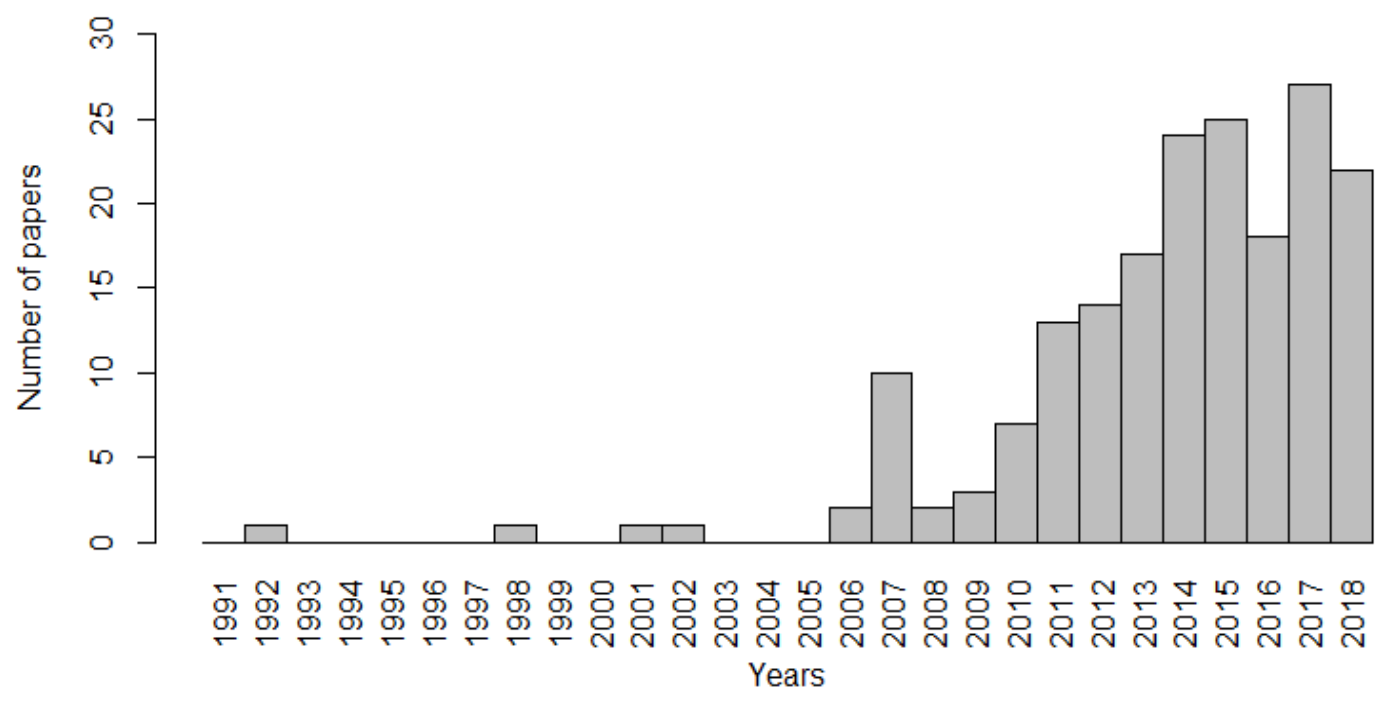

Figure 1.2. Papers on trait-based models included in the final list of reviewed models (Appendix. Tab. A1.1).

Types and scales of trait-based models

We classified the papers discovered in our systematic search according to model type and target scale. For model type, we distinguished among conceptual models, statistical models, equation-based models, individual-based models, and their combinations (see Glossary and Appendix. Tab. A1.1). Statistical models occurred in $26 \%$ and equation-based models in $61 \%$ of the reviewed papers. Together they were the most common types in trait-based modelling. Conceptual models are probably more common than was reflected in the papers we examined (5\% of the reviewed papers) because they often precede a mathematical or code-based model formulation. Individual-based models represented $16 \%$ of the reviewed papers.

Trait-based models address questions at local to landscape and global scales (Appendix. Tab. A1.1) and at the organizational level of individuals, species, populations, communities, and ecosystems. Where the models targeted the ecosystem level, they were implemented as equation-based models. This is probably due to the fact that ecosystem-level models focus on matter or energy fluxes and individual-based models are usually not the first choice for modelling fluxes, because this would require one flux equation per individual. However, models at the species, population or community level do not usually consider fluxes but use organisms as their inputs. This is typical of individual-based models but all other model types are also used at species, population, and community levels. Models 
of processes at the level of individual organisms or their organs were either implemented as statistical models of plant growth or, when emphasizing physiological mechanisms, as equation-based models.

Overall, different model types benefit in different ways from the integration of traits depending on the target scale of the question addressed by the model. In the following sections, we present studies that illustrate the potential benefits of using trait-based modelling for various scales and model types to study plants and animals in terrestrial and aquatic ecosystems, microbial organisms, and soil decomposers.

\subsection{Glossary}

Functional traits are well-defined morpho-physio-phenological characteristics of individual organisms that relate to the patterns of growth, reproduction, and survival of the species (McGill et al., 2006; Violle et al., 2007), and that evolved in response to abiotic environmental conditions and interactions with other species (Reich et al., 2003; Clark et al., 2012). As proxies of organismal strategies functional traits are differently distributed across environmental gradients. This variation in distribution may be also shaped by trade-offs among traits (Reich et al., 2003).

Hard traits are directly related to important physiological processes that define the growth, reproduction and survival of an organism. Hard traits are usually hard to measure, and therefore in practice they are identified and measured on the basis of surrogate soft traits (Hodgson et al., 1999) that are correlated with hard traits but are more easily or cheaply measured.

Response traits determine how a species reacts to a disturbance or a change in abiotic or biotic processes in its environment (Lavorel et al., 1997; Lavorel and Garnier, 2002).

Effect traits determine how a species influences ecosystem properties (Lavorel et al., 1997; Lavorel and Garnier, 2002). Effect traits alter abiotic and biotic processes corresponding to a wide range of ecosystem functions (Eviner and Chapin III, 2003).

Plant functional types (PFT) are groups of species with presumably similar roles in ecosystem functioning (Lavorel et al., 1997). They are considered as an important ecological framework for describing the mechanisms underlying vegetation responses (McIntyre et al., 1995; Pausas, 1999). 
Community-weighted mean (CWM) traits provide a quantification and use of aggregated trait attributes of the community as a measure of diversity that does not take species into account. To calculate a community aggregated trait value, relative abundances of species and their trait values are used (Violle et al., 2007; Funk et al., 2016).

Intraspecific trait variability (variation) is the difference in the values of functional traits within one species that results from the development and adaptation of species to environmental change (Albert et al., 2011; Schirpke et al., 2017). There are two sources for this variation. One is heritable differences between individuals and the other phenotypic plasticity in trait values across different environmental conditions (Moran et al., 2016).

Conceptual models are not implemented in equations or programming code. In practice, they are usually a graphical representation of causal relationships (or flows) between factors or processes.

Statistical models are descriptive mathematical models of relationships between variables based on assumptions about the data sampled. They represent a set of probability distributions on the sample space (Cox et al., 1979).

Equation-based models are mathematical models that are formulated as a set of ordinary differential equations, partial differential equations, or integro-differential equations. They can be solved analytically or numerically. These models are sometimes also called mechanistic models, physiological models or process-based models, although each of these terms is also used for nonequation-based models. For instance, process-based models are based on a theoretical understanding of the relevant ecological processes. They are built on explicit assumptions about how a system works, and these models are especially well-designed to predict the effects of global change (Cuddington et al., 2013). Dynamic Global Vegetation Models (DGVMs) and Earth System Models (ESM) also fall in this category.

Individual-based models (or agent-based models) explicitly consider individual organisms as objects with characteristics (traits) that influence interactions with other individuals and the environment (Grimm and Railsback, 2005). They adopt a bottom-up approach where population-level behaviour emerges from these individual interactions (DeAngelis and Grimm, 2014). Individual-based models are usually not based on equations, but on rules implemented in programming code. 
Individual-based models are highly suitable for spatially explicit implementations (Grimm et al., 2005), often combined with a grid-based modelling approach. Individual-based models are inherently linked to trait-based approaches, because interactions are mediated by traits in individual-based models (Fig. $1.4)$.

Trait-based models consist of combinations of functional traits that respond to environmental changes (response traits) and affect community and ecosystem properties (effect traits). Models based on discrete functional types or functional groups are not part of our definition of trait-based models, which consider continuous trait values.

\subsubsection{Trait-based modelling of plants in terrestrial ecosystems}

Trait-based approaches were originally developed and discussed for plants in terrestrial ecosystems. This focus on plant sciences was mirrored in the trait-based modelling studies. Fifty percent of all studies in this review addressed terrestrial vegetation (note that we discuss the two studies on fungi in our review as part of this section). The aims of trait-based vegetation models were diverse. They covered investigations of plant growth and interactions, species distributions, plant invasiveness, community assembly and dynamics, biodiversity hypotheses, ecosystem services, and global vegetation patterns and dynamics (Fig. 1.3).

\subsubsection{Trait-based models on plant growth, population dynamics, and interactions}

Plant growth, population dynamics, and interactions were modelled with a range of model types, including statistical, equation-based and individual-based models (Appendix. Tab. A1.1). The influence of traits on the growth of individual plants or plant organs was most commonly addressed using statistical models. These statistical models were either non-linear regression models (Chavana-Bryant et al., 2017), Bayesian approaches, or both (Hérault et al., 2011; Aubry-Kientz et al., 2015; Thomas and Vesk, 2017a; Thomas and Vesk, 2017b). Equation-based approaches focused on mechanisms such as carbon and biomass fluxes within and across plants (Enquist et al., 2007; Sterck and Schieving, 2011), water uptake (Fort et al., 2017) or on the physiological processes producing salt tolerance (Paleari et al., 2017). 
Both models on plant population dynamics in our review were equation-based models. One study investigated the influence of considering whole life cycles in fitness assessments (Adler et al., 2014), and the other one studied the population dynamics and viability of a primrose (and a lizard) population (Jaffré and Le Galliard, 2016). Comparing the results from the equation-based integral projection model with those from an analogous individual-based model, Jaffré and Le Galliard (2016) highlighted the importance of constructing individual-based models when very small populations are investigated. Our review confirms the conclusion of Salguero-Gómez et al. (2018) that trait-based approaches are still underrepresented in studies of population dynamics. However, note that such combinations of trait-based and demographic approaches are more common for aquatic organisms (Vindenes et al., 2014; O’Farrell et al., 2015) or terrestrial mammals (Santini et al., 2016; Jaffré and Le Galliard, 2016; van Benthem et al., 2017) than for plants.

Interactions such as competition have mainly been studied with equation- and individualbased models or their combination. Using dynamic process-based models, Ali et al. (2013) and Ali et al. (2015) contrasted two alternative competition theories and demonstrated how elevated carbon dioxide concentration influences plant competition and, consequently, community composition in an ecosystem. In a combined equation- and individual-based approach, Fyllas et al. (2014) simulated ecosystem fluxes based on two axes: the leaf economics spectrum (Wright et al., 2004) and tree architecture

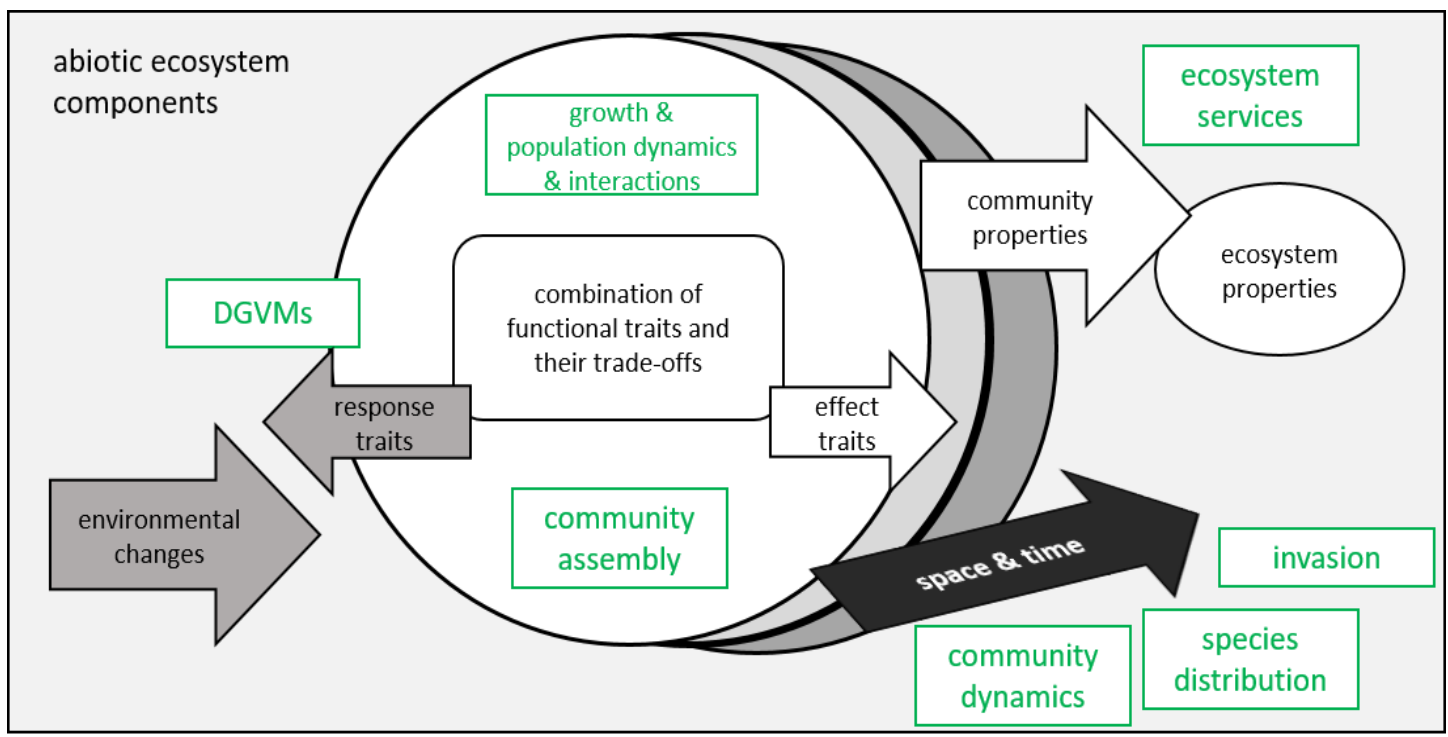

Figure 1.3. Overview of the models of plants in terrestrial ecosystems based on their main purposes and research questions (rectangles with green edges). Large circles represent community development in space and time and the rounded rectangle represents all traits and their interactions in the model (see Fig. 1.1 for further explanation). 
spectrums (Chave et al., 2005; Mori et al., 2010). Individual-based models are particularly useful for representing plant interactions because it is the individual level at which interactions are initiated. For example, Taubert et al. (2012) used them to investigate biofuel production in grasslands of temperate regions. In this model, above- and below-ground plant functional traits were used to characterize how successful plants were in taking up resources and competing with neighbours.

Conclusion: Based on these examples and the nature of the models, we suggest that different kinds of models have different efficiencies in the sense of producing good results without requiring large amounts of data. Statistical models are most efficient for describing the relationship between traits and plant growth but equation-based models are most efficient for describing mechanisms, simple interactions, or ecosystem fluxes. Individual-based models are the best choice, however, for complex trait-based interactions and for very small populations (see also Jaffré and Le Galliard, 2016). This is because individuals are the nexus of trait-based interactions.

\subsubsection{Trait-based models on species distributions}

Although trait-based modelling was often applied as an alternative to species-based approaches, a number of trait-based modelling papers were devoted to questions related to species distribution. For example, a combination of species distribution models and trait-based approaches was explicitly recommended to estimate the vulnerability of species to climate change with respect to selected species traits (Willis et al., 2015).

The great majority of the studies on species distribution modelling combined a trait-based approach with statistical modelling, often by using a Bayesian approach (Appendix. Tab. A1.1). For example, Powney et al. (2014) showed that predictions of trait-based species distribution models were best for broad-scale changes in regions with similar land-cover composition. Here, the trait-based approach was implemented by identifying traits that correlated with changes in species ranges and using these trait correlations to predict change in other regions. In another example, a new application of time-to-detection modelling was able to detect multiple species as a function of plant morphological 
and phenological traits (Garrard et al., 2013). The model by Rosenfield and Müller (2017) estimated the relative abundances of species that meet the values of functional traits found in a target ecosystem.

Trait-based models provide some advantages when predicting local community assembly, especially where environmental filtering and niche differentiation shape communities. Among the algorithms used in the papers on trait-based models, MaxEnt (e.g. Shipley et al., 2011; Sonnier et al., 2010) and the Traitspace model (e.g. Laughlin et al., 2012; Laughlin et al., 2015; Laughlin and Joshi, 2015) were used for trait-based environmental filtering. These algorithms predict low probabilities for any species whose trait distribution fails to pass through an environmental filter (Laughlin and Laughlin, 2013). The MaxEnt model and the Traitspace model differ in their ability to predict the relative abundance of species from a regional species pool (Laughlin and Laughlin, 2013). MaxEnt predictions are degraded when high intraspecific variability is included (Merow et al., 2011). The importance of intraspecific variation in functional traits was underlined by Violle et al. (2012) and by Read et al. (2017) who found that intraspecific variability compensated for the effects of interspecific variation along a climatic gradient. Therefore, future models should consider to address intraspecific variability - at least where the respective data are available. A statistical approach uniting trait-based and species distribution models was also applied to model the trait-based response and distribution of woodinhabiting fungi with respect to environmental change (Abrego et al., 2017). Finally, the only equation-based model in this section was a model that incorporated plant physiology to predict tree distributions along resource gradients (Sterck et al., 2014).

Conclusion: Thus, trait-based approaches are useful for modelling species distributions, especially where environmental filtering and niche differentiation are the predominating mechanisms. Of course, trait-based approaches would be even more suitable to model trait distributions. However, conservation managers are still more interested in species than in traits, so that a focus on trait distribution modelling is still less applicable. Based on the available examples, we locate the cutting edge of traitbased species distribution modelling in moving from mean trait values to intraspecific trait variability, e.g. by implementing a Bayesian framework (Laughlin et al., 2012). 


\subsubsection{Trait-based models of community assembly}

Community assembly results from species sorting by environmental filters and biotic interactions. Until now, trait-based models of community assembly used mainly statistical modelling and equationbased approaches to capture this process (Appendix. Tab. A1.1). We further found one individualbased model [(Pachepsky et al., 2007) based on a model by Bown et al. (2007) mentioned in section 1.1. Trait-based models on plant growth, population dynamics, and interactions], and three conceptual models (Bhaskar et al., 2014; Crowther et al., 2014; Losapio and Schöb, 2017), including one on fungal community assembly (Crowther et al., 2014). The group of models of community assembly partially overlapped with models of species distribution and was thus already partly discussed in section 1.2.Trait-based models on species distributions. Three groups of studies emerged: First, a large group of publications where the intention was to identify traits that affect community assembly; second, a group of four papers studying intraspecific trait variability (Pachepsky et al., 2007; Laughlin et al., 2012; Yang et al., 2015a; Schliep et al., 2018); and, third, another group of three papers where traits were used as response traits to distinguish between biotic and abiotic filtering (Bhaskar et al., 2014; Chauvet et al., 2017) and to assess effects of environmental change (Losapio and Schöb, 2017).

The majority of the models reviewed in the current section were in the first group that aimed to distinguish traits that influence species abundance, richness and functional diversity. One case study, for instance, indicated that seed production and dispersion traits are important for regional species abundance (Marteinsdóttir, 2014). In another case study, a trait-based model incorporated both neutral theory and niche theory to identify whether, and which, plant traits determine community assembly and biodiversity patterns, including plant species richness and abundance, across environments (Shipley et al., 2006). To unify classic coexistence theory and evolutionary biology with recent traitbased approaches, Laughlin et al. (2012) incorporated intraspecific trait variation into a set of traitbased community assembly models. These models generate species abundances to test theories about which traits, which trait values, and which species assemblages are most effective for achieving a specified functional diversity. Larson and Funk (2016) advocated for including regeneration traits in a model of community assembly. 
A few statistical trait-based models in the first group aimed at quantifying the relationship between environmental gradients and individual-level traits or community-weighted mean traits to describe environmental filters (Laughlin et al. 2015). The common assumption of such models was that traits are unimodally distributed and centred on an optimal trait value in any given environment. In contrast, an extended Traitspace model (Laughlin et al., 2015) adopting a hierarchical Bayesian approach (Laughlin et al., 2012) captured multimodal trait distributions. Improving the Traitspace model in this way increases the power of trait-based predictions of species abundances. The power increase arises because the prediction of species abundance distributions then reflects the true functional diversity of a community. These community assembly models were also used to test the mass ratio hypothesis (Laughlin, 2011; Laughlin, 2014) and to refine restoration objectives, either by manipulating abundances of species already existing in the system or by adding species from warmer climates to the local species pool (Laughlin et al., 2017). Similar to models on species distribution, the MaxEnt algorithm is also used for models of community assembly to predict the effect of trait-based environmental filtering on the species pool, for example, in forest community assembly (Laughlin et al., 2011) or using the community assembly via trait selection approach (CATS) (Laliberté et al., 2012; FrenetteDussault et al., 2013).

Equation-based models in the first group tended more towards theoretical questions: For example, they investigated the multidimensional nature of species coexistence based on traits (Kraft et al., 2015), they implemented biophysical principles to test niche vs neutral processes (Sterck et al., 2011); or they showed that self-limitation promotes rarity (Yenni et al., 2012).

Conclusion: The examples in this section show that trait-based modelling is a versatile tool to investigate mechanisms and effects of community assembly due to the availability of response and effect traits, the focus on function, and the possibility to study mechanistic detail by including intraspecific trait variability. The diversity of examples also emphasizes the suitability of trait-based community assembly models for tackling questions of fundamental and applied ecology. 


\subsubsection{Trait-based models of community dynamics}

Trait-based modelling can be helpful for explaining not only static community assembly but also the temporal and spatial dynamics of communities. We found twice as many equation-based models of community dynamics as individual-based models (Appendix. Tab. A1.1). The equation-based approaches included basic and applied research. Among the basic research, two studies investigated vegetation dynamics at the landscape level (Falster et al., 2011; Quétier et al., 2011), one study quantified environmental filtering and immigration rates of new species (Jabot, 2010), and one study assessed plant community stability considering litter decomposition (Miki and Kondoh, 2002). Among the more applied research, three studies included the effect of environmental change: Moor (2017) studied the relationship between dispersal and species diversity along a climate warming gradient; Savage et al. (2007) investigated overyielding and other responses to environmental change; and Tanaka (2012) advanced Savage et al.'s (2007) study by considering interspecific competition and trait covariance structure. One further equation-based model with an applied question was developed to compare the effect of different cropping systems on weed traits (Colbach et al., 2014).

The individual-based models in this section targeted effects of regional processes on grasslands (Weiss et al., 2014), as well as the processes of grazing and disturbances. The effects of grazing on a grassland community were investigated in two individual- and trait-based models (May et al., 2009; Weiss and Jeltsch, 2015). The results of these models indicated that trait size symmetry of competition is central for community dynamics. This indication arose from the model only generating the patterns predicted by the grazing reversal hypothesis under specific conditions. These conditions were the explicit inclusion in the model of shoot and root competition, and the assumptions that plants with larger aboveground parts were superior competitors and belowground competition was consistently symmetrical. A similar functional group scheme based on four key traits representing typical species responses to disturbance was used in an individual-based model that led to the conclusion that the competition-colonization trade-off is insufficient to predict community dynamics (Seifan et al., 2012; Seifan et al., 2013). 
Conclusion: The reviewed studies in this section show that the potential application areas are more fully covered by equation- than individual-based approaches. These studies also show the great ability of trait-based models to capture the mechanisms that drive plant interactions and their impact on community dynamics. Beyond the current focus on grassland communities of individual- traitbased models, we suggest that desert, savanna and forest community dynamics should be explored in future studies. Furthermore, although temporal changes in a community are often accompanied by spatial changes and spatial interactions are often mediated by traits, our review shows that there is still some unused potential in modelling spatial community dynamics based on traits.

\subsubsection{Trait-based dynamic global vegetation models (DGVMs)}

Beyond the community and ecosystem levels mentioned in previous sections, global vegetation classification is possible with DGVMs. More specifically, DGVMS advance understanding of the distribution of plant functional types across spatial scales (Prentice et al., 2004). DGVMs are used as precursors of, or parts of, earth system models in which they represent energy, carbon and water fluxes (Scheiter et al., 2013; Drewniak and Gonzalez-Meler, 2017). Dynamic vegetation classification is enabled by calculating separately ecosystem fluxes and plant functional type occurrences both of which can be based on traits. DGVMs are mainly or entirely equation-based models because this form allows them to adequately represent ecophysiological processes (Appendix. Tab. A1.1). More recent DGVMs have added individual-based components to account for individual variation (Scheiter et al., 2013).

Most DGVMs were used to investigate vegetation responses to current climate and climate change (e.g. Verheijen et al., 2013; Sakschewski et al., 2015). Walker et al. (2017) applied the Sheffield DGVM (Woodward and Lomas 2004) to compare the predictive power of four trait-scaling hypotheses on the distribution of global maximum rate of carboxylation. The four hypotheses used were those on plant functional type, nutrient limitation, environmental filtering, and plant plasticity. The result of this comparison showed that nutrient limitation was the most probable driver of global maximum rate of carboxylation distributions. DGVMs are criticized for being insufficient realistic. This insufficiency arises because they use plant functional types with constant attributes and do not repre- 
sent competitive interactions (Scheiter et al., 2013). This criticism led to a stronger focus on traits and to the addition of individual-based modules (Harper et al., 2016). The Jena Diversity-DGVM incorporates 15 traits with several functional trade-offs that define plant growth strategies (Pavlick et al., 2012). These functional properties of the vegetation were derived, unlike in standard DGVMs, from mechanistic trait filtering via environmental selection. The Jena Diversity-DGVM also demonstrated its advantages over bioclimatic approaches (Reu et al., 2010; Reu et al., 2011). Instead of plant functional types, the adaptive DGVM of Scheiter and Higgins (2009) and Scheiter et al. (2013) was based on traits. The novelty of this adaptive DGVM lay in the process-based and adaptive modules for phenology, carbon allocation and fire within an individual-based framework. This allowed the vegetation component in the model to adapt to changing environmental conditions and disturbances. Such adaption is not possible in models based on static functional types.

Conclusion: As our review demonstrates, DGVMs provide a good example of the shift from plant functional types towards functional traits (Yang et al., 2015b) (e.g. compare Smith et al. (2001) and Holzwarth et al. (2015)). The reviewed studies give examples of how adaptive, flexible and realistic trait-based models can be, emphasizing their strengths in these attributes. Moreover, individualbased modelling is increasingly applied to represent individual interactions and foster the dynamic nature of DGVMs.

\subsubsection{Trait-based models of plant invasions}

Trait-based modelling was frequently used to study invasion (Appendix. Tab. A1.1). The frequency of this use probably arises because it is a common goal of invasion biology to identify traits that can be used to predict future invaders. This goal was particularly common among statistical models (Otfinowski et al., 2007; Herron et al., 2007; Küster et al., 2008). The individual-based models of plant invasion focused on understanding the invasion process incorporating, for instance, disturbance (Higgins and Richardson, 1998), or herbivory (Radny and Meyer, 2018). These models have different regional and taxonomic foci including pine trees in the southern hemisphere (Higgins and Richardson, 
1998), exotic plants in North America (Otfinowski et al., 2007; Herron et al., 2007), invasion success in Germany (Küster et al., 2008), and establishment success as the combined effect of functional traits and biotic pressures (Radny and Meyer, 2018).

Conclusion: While usage of trait-based modelling in the study of invasions is growing, these models have yet to yield a universal set of traits that characterize potentially invasive species. Future trait-based invasion models should address all the processes and interactions relevant to the system being studied. This might be facilitated by individual-based modelling approaches, as the broad range of individual-based models in this section demonstrates. The models of plant invasions may also benefit from the advantages discussed in Section 1.4.1.4. Trait-based models of community dynamics.

\subsubsection{Trait-based models of ecosystem services}

Ecosystem service models are usually built with a management goal. Thus, they benefit from including plant functional traits because functional traits are aggregate measures that can more easily be targeted by ecosystem management than species. Most models in this section were statistical models (Appendix. Tab. A1.1), often in the form of generalized linear models (Diaz et al., 2007; Lavorel et al., 2011), whereas three models were equation-based. According to Lavorel et al. (2011), ecosystem properties were better captured by models including spatial variation in environmental variables and plant traits than by land-use models. Variation across the landscape in the community-weighted mean of four traits and their functional divergence were modelled with generalized linear models (Lavorel et al., 2011). Compared to remote sensing, this trait-based statistical modelling approach better reflected the process of land use that underlay ecosystem properties (Homolova et al., 2014). Another model investigated the influence of plant and microbial functional traits on grassland ecosystem services (Grigulis et al., 2013). Based on Lavorel et al. (2011) and Grigulis et al. (2013), future ecosystem services were estimated for three socio-economic scenarios (Schirpke et al., 2017). The approach demonstrated that ecosystem services were potentially highly resilient. In two other semimechanistic models, 
functional traits facilitated the scaling-up of well-understood functional trade-offs from the organismal to the ecosystem level (Lamarque et al., 2014).

Equation-based models of ecosystem services ranged from assessing the sensitivity of ecosystem services to land-use change (Quétier et al., 2007), determining the vulnerability of pollination services (Astegiano et al., 2015), and evaluating the management of mown subalpine grasslands (Lochon et al., 2018).

Conclusion: Based on the examples in this section, trait-based models demonstrate great potential for solving applied questions in ecosystem studies as well as for those involving scaling. Traitbased models are particularly advantageous to explore ecosystem services because of the fact that traits help identify underlying mechanisms such as land-use change.

\subsubsection{Trait-based models on interactions between plants and other organisms}

A few trait-based models did not focus purely on vegetation and interactions among plants but also included the interactions of plants with other groups of organisms. These models were partly conceptual and partly equation-based (Appendix. Tab. A1.1). For example, the effects of biodiversity on multispecies interactions and cross-trophic functions were described in a trait-based bottom-up framework (Lavorel et al., 2013). This conceptual model was linked to a statistical structural equation model, which demonstrated that high functional and interaction diversity of animal mutualists promoted the provisioning and stability of ecosystem functions. In another case study, avian body size was identified as an important response trait related to the susceptibility of avian seed dispersers to disturbance by humans (Schleuning et al., 2015). A conceptual model (Pöyry et al. 2017) reconstructed how the effects of soil eutrophication cascade to higher trophic levels across a range of plant-herbivore interactions. The model was evaluated based on butterfly and moth data. The authors suggested that a major future trend will be the increased dominance of insect species that are large, dispersive dietary generalists over those preferring oligotrophic environments. These conceptual models await further testing by 
being converted into equation- or code-based models and the empirical testing of the predictions of these models.

There were also four equation-based models in this section addressing nutrient competition in an earth system model (Zhu et al., 2016), trade-offs of defensive plant traits in plant-herbivore interactions (Mortensen et al., 2018), plant-soil feedback mediated by litter and microorganisms (Ke et al., 2015), and three-way interactions between a plant, a herbivore and a beneficial microbe in the context of biological invasions (Jack et al., 2017).

Conclusion: Due to the fact that interactions are mediated by traits, trait-based models are ideal to capture a variety of conceptual interactions, inclduing the ecologically significant cases of interactions between plants and other organisms.

\subsubsection{Trait-based modelling of animals in terrestrial ecosystems}

Animals are underrepresented among papers on the trait-based modelling of terrestrial ecosystems, whereas trait-based models of marine ecosystems which included animals abounded. There are about three times as many marine as terrestrial papers including animals in our review (see section 1.4.3. Trait-based modelling in aquatic ecosystems). The reason for the imbalance may lie in there being many different behaviours, feeding strategies and morphologies among terrestrial animals (Scherer et al., 2016), making it hard to define common functional traits. Nevertheless, 23 papers covered traitbased modelling of animals in terrestrial ecosystems. There were twice as many equation-based models as individual-based models and almost as many statistical models as equation-based ones (Appendix. Tab. A1.1). As the following studies show, these models cover a broad range of topics, including population dynamics and survival analysis, predator-prey and host-pathogen interactions, species distributions, and community assembly.

Population dynamics was more frequently investigated in trait-based modelling studies of animals than plants in terrestrial ecosystems. We included here pure population dynamics studies, but also other studies at the population level, namely time-to-detection studies, survival analysis, vulnerability analysis, home range determination, and a study of eco-evolutionary dynamics. For example, the effect of functional traits on the population dynamics of mites was studied with equation- 
based integral projection models (Smallegange and Ens, 2018), drawing on the dynamic energy budget theory better known from aquatic studies (see section 1.4.3. Trait-based modelling in aquatic ecosystems). According to an individual-based model, the population dynamics of meerkats depend on intraspecific variation in body mass (Ozgul et al., 2014). In a similar but equation-based model traitdemography relationships were studied to identify the mechanism underlying population fluctuations (van Benthem et al., 2017). Trait-based models of population dynamics investigated the responses of populations to environmental changes (Santini et al., 2016) and to perturbations (Ozgul et al., 2012). Using an approach similar to that of the time-to-detection studies mentioned in section 1.4.1.2. Traitbased models of species distributions, Schlossberg et al. (2018) modelled detectability for ten mammal species. This model was based on species traits such as body mass, mean herd size and colour and employed a statistical approach based on conditional likelihoods. An example of a trait-based survival model was the prediction of bat survival based on reproductive, feeding, and demographic traits such as age, sex, and type of foraging (Lentini et al., 2015). A trait-based vulnerability index was applied to subarctic and arctic breeding birds in a statistical model constructed around MaxEnt and CATs (Hof et al., 2017). We found three further individual-based models: Scherer et al. (2016) explored the response of bird functional types to climate and land-use change; Buchmann et al. (2011) used the methodology to predict the home range and the spatial body mass distribution of species in terrestrial mammal communities in fragmented landscapes; And, for a theoretical study of eco-evolutionary dynamics, Pontarp and Wiens (2017) simulated the evolutionary radiation of a clade across several habitats with differing environmental conditions.

Predator-prey interactions were considered from a functional perspective relatively early on in the history of trait-based approaches, i.e. when generalist and specialist functional types were introduced into modelling (Hanski et al., 1991). Functional traits are a much more recent characteristic of predator-prey modelling studies, e.g. in a general additive model of beetle predation with eight predator traits and four prey traits (Brousseau et al., 2018). The novelty of these models is that the combination of functional traits and phylogeny overcome the limitations of purely descriptive approaches. Where predator-prey interactions are combined into a food web model, body size is often the central trait. This was the case in an allometric trophic network model that explicitly featured intra- and inter- 
specific interference including predator-prey interactions in beetles and spiders (Laubmeier et al., 2018). Pathogen-host interactions resemble predator-prey interactions in many ways as demonstrated by the interactions of amphibian species and their fungal pathogens. In this case, the interactions were modelled with a statistical approach investigating the predictive power of traits related to phylogenetic history, habitat use, and life history traits (Gervasi et al., 2017). Individual- and trait-based movement models are very powerful when it comes to scaling-up across several levels of organization. This feature was exploited in an individual-based model that scaled up from individual movement and behaviour to metacommunity structure (Hirt et al., 2018).

Species distributions and species niches were modelled for the cane toad with a statistical approach (Kearney et al., 2008; Kolbe et al., 2010) and for endotherms (Porter and Kearney, 2009) and ants (Diamond et al., 2012) with an equation-based approach. For the endotherms and the ants, biophysical principles were used to link variation in functional traits with environmental data to predict thermal niches (Porter and Kearney, 2009; Diamond et al., 2012).

Community assembly and dynamics were studied with equation-based models which, for example, accounted for spatial variation in community structure with a multi-region multi-species occupancy model (Tenan et al., 2017), investigated irreversible changes in community structure in a consumer-resource model (Haney and Siepielski, 2018), and used trait-mediated interactions to analyse invasiveness and invasibility of ecological networks (Hui et al., 2016). The need to include such process-based components in community assembly models was emphasized by Pontarp and Petchey (2016).

Conclusion: The models in this section show a great diversity of applications for the traitbased modelling of animals in terrestrial ecosystems, such as studying the influence of intraspecific variation in body mass on population dynamics, investigating the mechanisms underlying population fluctuations, exploring the response of populations to environmental change, simulating evolutionary radiation and scaling up metacommunity structure from individual behaviour. However, each topic is represented by one or very few studies. This indicates that there is scope for more applications in these and related fields, regardless of model type. 


\subsubsection{Trait-based modelling of aquatic ecosystems}

According to Litchman and Klausmeier (2008), the trait-based approach was first used for modelling aquatic ecosystems in a model of a phytoplankton community by Ramon Margalef (Margalef, 1978). Nevertheless, Follows and Dutkiewicz (2011), in their analysis of the state of the art of marine ecosystems, concluded that trait-based approaches were just then (i.e. in 2011) starting to be used in marine ecosystem models. The conflict between these two statements demonstrates different understandings of what a trait-based model is.

In addition to the research papers, we also found that reviews on trait-based approaches for studying aquatic ecosystems are not uncommon. Therefore, we shortly summarize the most important reviews here. Litchman et al. (2010) reviewed trait-based approaches applied to phytoplankton and revealed a new trend - to look at a trait and the phylogenetic structure of communities simultaneously. This trend, in combination with adaptive trait models, makes it possible to predict trait evolution. In another review on trait-based approaches for studying phytoplankton, Bonachela et al. (2016) showed that it is also possible to successfully use trait-based models to identify and compare possible survival strategies described by a set of functional traits. These models typically include trade-offs between traits such as cell-size and resource allocation.

In the following sections, we first review aquatic trait-based models including those for fish and then those focusing on plankton. There are few trait-based modelling studies of other aquatic realms, which are briefly covered in this paragraph, e.g. studies of bivalve species distribution models (Montalto et al., 2015), inland freshwater communities (Gardner et al., 2014), coral reefs (Edmunds et al., 2014; Madin et al., 2014), a pelagic microbial mixotrophic food web (Castellani et al., 2013), marine benthic communities (Alexandridis et al., 2017), diatoms in peatlands (Hagerthey et al., 2012), and trace metal concentrations in invertebrates (Hug Peter et al., 2018). Traits were usually the inputs for models but in one case were outputs (Rinaldi et al. 2014). In that paper, mechanistic functional trait models were used to predict life history traits such as body size and fecundity of shellfish in lagoons. The fact that traits were used both as inputs and outputs for models emphasizes the difference between 
effect traits and response traits (Fig. 1.1), two concepts introduced earlier to terrestrial ecological theory (Lavorel and Garnier, 2002).

\subsubsection{Trait-based models including fish}

Trait-based modelling is widely applied to model fish communities. Of those models, the overwhelming majority were equation-based models (Appendix. Tab. A1.1). There were only four individualbased models (Brochier et al., 2013; Houle et al., 2013; O'Farrell et al., 2015; Huebert et al., 2018), one statistical model (Howeth et al., 2016) and one statistical and conceptual model (Bennett et al., 2016) that generalised the trilateral life history model by Winemiller and Rose (1992). This prevalence of equation-based models may be due to the fact that aquatic ecosystems are more homogeneous than terrestrial ecosystems and therefore lend themselves more naturally to the continuous character of most equation-based models.

Size appears to be the main structuring trait in aquatic ecosystems because size influences the most important organism processes, such as foraging, growth, and reproduction. For example, fish fall into different trophic levels when young than when old. Because size usually correlates with age, trophic level in fish is linked to body size. Size structure prevails up to the community level in marine ecosystems. This fact prompted the formulation of the community size spectrum (Guiet et al., 2016b). The regularity of the community size spectrum is expressed in the constancy of total ecosystem biomass within "logarithmically equal body size intervals" (Guiet et al., 2016b). Thus, community sizespectrum models represent the ecosystem using two parameters - the slope and the intercept of the community size spectrum. This type of model mechanistically addresses the role of species diversity via the introduction of the trait size (Hartvig et al., 2011; Maury and Poggiale, 2013; Guiet et al., 2016a). In these models, community dynamics emerge from individual interactions. Trait-based sizespectrum models were developed with a range of goals, including to study the benefit to fish of the reproductive strategy of producing many small eggs or to analyse coexistence between species and link it to maturation sizes and predator-prey size ratios (Hartvig and Andersen, 2013). Trait-based size-spectrum models also demonstrate the impact of fishing on species composition (Shephard et al., 
2012), i.e. that fishing out larger individuals shifts the size spectrum towards the dominance of smaller species.

Originally, Andersen and Beyer (2006) introduced a size- and trait-based model to estimate fishing effects at the ecosystem level. In this model, every individual was characterized by two features: body size and asymptotic body size. This model was later expanded (Houle et al., 2013; Zhang et al., 2013; Jacobsen et al., 2014; Jennings and Collingridge, 2015). The asymptotic body size was defined as a main trait because it is the basis for applying life history theory to estimate size at maturity and reproductive output (Jennings and Collingridge, 2015). The indirect influence of fishing on community structure was revealed by an extended version of the initial model considering entire life histories and individual energy budgets (Kolding et al., 2016). Another size- and trait-based model included individual interactions in the form of competition and predation and individual processes such as encounters, growth, mortality and reproduction (Jacobsen et al., 2014). A similar model was developed by Andersen and Pedersen (2010) and Andersen and Rice (2010). In this model, all basic processes at the community level emerged directly or indirectly from individual-level processes. To answer the question how to maximize fishing yield under a certain conservation constraint, Andersen et al. (2015) suggested a conceptual size- and trait-based model. An adaptation and a dynamic version of the model of a theoretical fish community (Pope et al., 2006), based on classical multi-species fishery models and community size spectrum models, was reconsidered by Andersen and Pedersen (2010) and Andersen et al. (2015) in the framework of a trait-based approach. A similar model to describe population structure based on the size of the organisms was presented by Hartvig et al. (2011) and Hartvig and Andersen (2013). This model was a product of the synthesis between traditional unstructured food webs, allometric body size scaling, trait-based modelling, and physiologically structured modelling (Hartvig et al., 2011). These approaches were further developed into more complex food web models that showed that climate change effects are highly unpredictable (Zhang et al., 2014; Zhang et al., 2017). Using Approximate Bayesian Computation in their food web model, Melián et al. (2014) highlighted the importance of accounting for intraspecific variability when investigating species coexistence. Such combinations of traditional approaches with novel modelling techniques provides a promising new approach to the study of size-structured food webs. 
Other examples, which we briefly describe in this section, covered the topics of marine biodiversity exploitation, marine community modelling including seal species (Houle et al., 2016), adaptive behavioural responses, fish-mesozooplankton interactions, fish-jellyfish interactions and freshwater fish modelling. Marine biodiversity exploitation was studied with an object-oriented individual-based model (Brochier et al., 2013). This model incorporated four main categories of life history depending on which part of the life cycle fish spent in the estuary studied. To reduce the computing power needed, Brochier et al. (2013) used a super-individual approach (Scheffer et al., 1995) with one individual representing a fish school. They also created 15 groups of ecologically similar model species, each representing a group of real species (Ecoutin et al., 2010). Each group contained one or more superindividuals with similar trophic position and ecological traits. Persistent spatial interactions and cascading behavioural interactions were revealed in a marine ecosystem model with detailed size structure and life cycles of mesozooplankton and fish (Castellani et al., 2013). This model became a step towards a mechanistic and adaptive representation of the upper trophic levels in ecosystem models. In this model, the main trait was size at maturation. Based on a traditional ocean ecosystem model including chemistry, phytoplankton, micro- and mesozooplankton (Schrum et al., 2006), a new model version replaced the compound group of mesozooplankton by a developmental stage- and speciesspecific matrix and introduced fish feeding on mesozooplankton (Castellani et al., 2013). A general mechanistic food web model of fish-jellyfish competitive interactions was based on the feeding traits of fish and jellyfish populations (Schnedler-Meyer et al., 2016). The model also incorporated, in addition to feeding traits, elemental composition, allometric scaling of vital rates, locomotion, and lifehistory traits. The model predicted fish dominance at low primary production and a shift towards jellyfish with increasing productivity, turbidity and fishing.

A few freshwater studies included one on the simulation and screening of freshwater fish invasion which were tackled with the help of trait-based statistical models that used classification trees (Howeth et al. 2016). Another example investigated temperature-dependent colonization and extinction rates of darter fish in a body size-centred dynamic occupancy model (Shea et al., 2015). Stochastic integral projection models were not only used for plants and terrestrial animals, but also in a traitbased modelling study of pike in a freshwater ecosystem (Vindenes et al., 2014). 
Conclusion: Based on these publications, we believe there is no question that the long and successful history of trait-based modelling including fish centred on size spectrums will continue. Future applications are likely to further improve model predictions by following the increasing number of examples where traits other than size are also included in the models.

\subsubsection{Trait-based models focusing on plankton}

As with fish, it is also possible to explicitly model plankton in a trait-based way (Follows et al., 2007; Litchman et al., 2007; Bruggeman and Kooijman, 2007; Kiørboe, 2011). The models used are predominantly equation-based models, as they were for those including fish discussed in the previous section (Appendix. Tab. A1.1). Similarly, the models considered size as the main functional trait. Only two models were supplemented with individual-based modules (Clark et al., 2013; Pastor et al., 2018), two adopted a statistical approach (Litchman et al., 2007; Terseleer et al., 2014), and one a conceptual approach (Glibert, 2016). The great majority of these models targeted the ecosystem level. This focus on ecosystems is possibly also one of the reasons for the domination of equation-based approaches because such approaches are particularly well suited to capture ecosystem fluxes.

The marine ecosystem model by Follows et al. (2007) became a starting point for the development of a number of trait-based models in microbial ecology and plankton research. This is probably because it successfully reproduced the observed global distributions and community structure of the phytoplankton. The model included a diverse phytoplankton community that was described by a set of physiological traits defined by field and laboratory data with related trade-offs.

Phytoplankton cell size, and especially the drivers of small cell size, were addressed with a trait-based model of cellular resource allocation (Clark et al., 2013). This model considered a threeway trade-off between cell size, nutrient and light affinity, and growth rate. It was developed as a combination of a classic nutrient-phytoplankton-zooplankton model and 'cost-benefit' models. The trait-based approach was supported by individual-based modelling such that individual life histories gave rise to the evolutionary dynamics of the whole system. This bottom-up approach allowed missing ecosystem processes to be derived from model data. Where many individuals are similar, as in the 
general case of plankton and the specific case of this model (Clark et al., 2013), super-individuals can be created that represent groups of individuals with similar traits. As in other super-individual applications (e.g. Brochier et al., 2013 in section 1.4.3.1. Trait-based models including fish), this approach reduces computing power requirements. Plankton cell size and the mechanisms underlying observed biogeographical difference in cell size were also studied by Acevedo-Trejos et al. (2015) and Acevedo-Trejos et al. (2018). Their models considered trade-offs between cell size and nutrient uptake, zooplankton grazing, and phytoplankton sinking. Macroscopic system properties such as total biomass, mean trait values, and trait variance were studied with a continuous trait-based phytoplankton model (Chen and Smith, 2018). This model was developed as a sub-module of a larger model the goal of which was to simulate ocean dynamics. The model produced realistic patterns of phytoplankton mean size and size diversity. Co-evolution of traits with respect to chromatic and temperature adaptation was studied with a trait-based ecosystem model (Hickman et al., 2010). Trait-based models with adaptive traits were compared to trait-group resolving models in a study of phytoplankton communities in partially mixed water columns (Peeters and Straile, 2018).

Disease transmission in multi-host communities was the focus of a multi-generational plankton-based model that considered epidemiological traits such as foraging or exposure rate, conversion efficiency, susceptibility, virulence and spore yield (Strauss et al., 2015). This model succeeded in improving the mechanistic and predictive clarity of the dilution effect by connecting a reduction in diluter species with the increase in disease risk. The dilution effect probably explains links between host communities and transmission. In their model of virus infection of plankton based on life-history traits, Beckett and Weitz (2018) found that lysis rates were driven by the strains with the fastest replication and not those with the greatest abundance.

Compared to models with better resolved species-specific representations of physiological processes, improved representation of biodiversity was suggested in a biodiversity-based marine ecosystem model (Bruggeman and Kooijman, 2007). The model was based on a system of infinite diversity in which species were defined by continuous trait values for light-harvesting investment and nutrient-harvesting investment. The traits chosen affected all parts of the metabolism forming a trade-off 
between harvesting and net growth. Based on this model and a model by Bruggeman (2009), a traitbased model was developed to include mixotrophy, succession and evolution of unicellular planktonic organisms and to predict optimum trophic strategies of species under changing environmental conditions (Berge et al., 2017). This model contained three key resource-harvesting traits: photosynthesis, phagotrophy and inorganic nutrient uptake. To distinguish two different mixotrophic strategies, Chakraborty et al. (2017) extended the model by Berge et al. (2017) by explicitly incorporating cell size and introducing a pure heterotrophic strategy.

Different aspects of plankton ecosystems were recently scrutinized at greater detail, including trait-based ecosystem function predictions for a global lake data set (Zwart et al., 2015), biological interactions, species extinctions, nutrient uptake kinetics, and some theoretical properties as well as more applied implications of plankton models. Interactions were investigated in the form of temperature dependence of competition of phytoplankton species (Bestion et al., 2018) and of host-pathogen interactions between zooplankton and a fungal pathogen, which seem to be mediated by host foraging under climate warming (Shocket et al., 2018). Species extinctions strengthen the relationship between biodiversity and resource use efficiency (Smeti et al., 2018) based on a model studying phytoplankton succession (Roelke and Spatharis, 2015a) and assemblage characteristics (Roelke and Spatharis, 2015b). Based on insights from a size-based model on nutrient uptake kinetics of phytoplankton, Smith et al. (2014) emphasized that plankton ecology benefits from mechanistic trait-based models that account for physiological trade-offs. In a theoretical exercise, Gaedke and Klauschies (2017) showed that the knowledge of the shape of observed trait distributions is beneficial for the elegant analysis of aggregate plankton models, because it allows for data-based moment closure. With a new scale-invariant size-spectrum plankton model, Cuesta et al. (2018) explored the constancy of the relationship between biomass density and logarithmic body mass across scales. Finally, there were examples of trait-based plankton models that explicitly addressed applied questions such as the management of harmful algal blooms (Glibert, 2016; Follett et al., 2018).

Conclusion: The vibrant field of trait-based plankton models is a good example of how traitbased approaches can inspire ecosystem modelling. The relatively homogeneous conditions in aquatic 
environments lead to the dominance of one trait - size - over any other trait or any taxonomic category in explanations of community and ecosystem processes and patterns. Although size as a main trait promotes simplification, which is the core aim of models, size is not always the ideal trait to describe all processes relevant for aquatic organisms. Thus, inclusion of further traits such as light and nutrient affinity (Bruggeman and Kooijman, 2007; Clark et al., 2013; Acevedo-Trejos et al., 2015; Berge et al., 2017) is required to improve models that aim to address such processes. Due to the central role of body size and other traits for processes in aquatic realms, trait-based approaches are more suitable than species-based approaches to model aquatic communities and ecosystems.

\subsubsection{Trait-based models on microorganisms and soil decomposers}

Microorganisms and soil decomposers are relatively new subjects of trait-based modelling and are still often represented as functional groups or functional types rather than traits. Therefore, the following seven examples also included classifications into functional groups. As in models of aquatic ecosystems, the models of microorganisms and soil decomposers were built around the key trait body size and were predominantly equation-based (Appendix. Tab. A1.1) with the exception of one statistical model (Van Bellen et al., 2017). In terms of scale, all but one model in this section operated at the community level. This one exception targeted continental to global scales (Wieder et al., 2015). Such scales are surprisingly large for a model including microbial processes.

Nitrification by ammonia-oxidizing bacteria, ammonia-oxidizing archaea and nitrite-oxidizing bacteria was considered in a mechanistic trait-based model (Bouskill et al., 2012). It was based on traits connected to the enzyme kinetics of nitrite. Another version of this model simulated the influence of global change on ecological niches of soil nitrite-oxidizing bacteria types (Le Roux et al., 2016). This trait-based model grouped nitrite-oxidizing bacteria into a few functional groups. The authors demonstrate that this approach was successful because three main bacterial functional types expressed contrasting responses to environmental changes.

Using functional types can be inferior to using functional traits. This was demonstrated by another microbial model that addressed time lags in the enzymatic response of denitrifying microorgan- 
isms to changes in substrate concentration, including the interactive dynamics between enzymes and nutrients (Song et al., 2017). This model linked community traits with functional enzymes, not species or functional guilds as in previous studies (Taffs et al., 2009; Bouskill et al., 2012). With organisms whose multiple functions overlapped with one another, the guild-based (functional type) approach failed to properly represent these organisms. Enzyme- and thus trait-based implementations provide tools for scaling up biogeochemical functions to the community level without involving the dynamics of individual species or their guilds.

A physiological trade-off between the traits of drought tolerance and carbon use efficiency was at the core of two modelling studies on soil decomposition (Allison, 2012; Allison and Goulden, 2017). In these models, the decomposition submodel of enzymatic traits was derived from the phytoplankton model by Follows et al. (2007) to predict litter decomposition rates in soil. The sensitivity of microbial traits, community dynamics, and litter decomposition to variation in drought tolerance costs was quantified in an updated model (Allison and Goulden, 2017). The model implied that, for the Mediterranean climate system, seasonal drought was a more important environmental filter than reduced precipitation during the wet season. These models were examples of successful exchange between disciplines.

Conclusion: Trait-based models are not yet so common for microorganisms and soil decomposers, but the few examples show their great potential for future applications. One example (Song et al., 2017) also illustrated nicely how important it can be to use functional traits instead of functional types.

\subsubsection{General insights on trait-based modelling}

There were few general trait-based models that are applicable to terrestrial and marine ecosystems alike. One of the rare cases, Harfoot et al. (2014), was a general ecosystem model based on eight traits thought to be the most important for determining rates of ecological processes. These traits were realm, nutrition source, mobility, leaf strategy, feeding mode, reproductive strategy, thermoregulation mode, and body mass. This model benefited from a coupled individual- and equation-based approach. The equation-based approach was applied to autotrophs and the individual-based one to all other or- 
ganisms. In this way, ecosystem structure and function emerged from interactions at the individual level. However, the individuals in this model were in fact groups or cohorts of organisms (the superindividual approach, Scheffer et al., 1995). This general ecosystem model showed that highly complex models require the combination of different modelling approaches including simplification tools such as the super-individual approach.

All in all, the variety of models in all sections demonstrates that trait-based modelling approaches are useful tools that are able to facilitate modelling and improve the predictive power of model outcomes across taxa and disciplines (Suding and Goldstein, 2008; Litchman et al., 2010; Powney et al., 2014; Laughlin et al., 2015; Song et al., 2017). In this variety of models, we nevertheless see a number of trends that allows comparison and incorporation of results across taxa and disciplines. One of these is a shift from functional types to functional traits. There is also a search for generalizations across organisms with similar functions and a few examples that scale-up processes from the local to the global level. Techniques that appear to be useful, especially in individual-based models, are the super-individual approach and the incorporation of intraspecific trait-variability. Nevertheless, it seems clear from both empirical and modelling studies that the development of trait-based models did not produce a universal set of traits. Therefore, researchers should instead select traits according to the research question and strategies of the organisms under investigation.

\subsection{Discussion and Conclusions}

Based on our systematic review, it is clear that trait-based approaches are as valuable in modelling studies as they were earlier in empirical studies. They facilitate parameterization and scaling-up of models as well as the generalization of their results. Despite some inconsistencies in the terminology of trait-based studies, trait-based models have been implemented widely for different groups of organisms and ecosystems, in different model types, and for achieving a broad range of aims (See Appendix. Tab. A1.1). We observed productive exchange of trait-based modelling concepts and 
techniques, especially between vegetation ecology and other disciplines, and argue that this should be intensified and extended to more disciplines in the future.

Inconsistencies in terminology within trait-based approaches mainly originate from the unclear differentiation between functional types and functional traits as categories for grouping organisms. For example, Jeltsch et al. (2008) suggested three strategies for applying plant functional type approaches in modelling, where the "functional trait" strategy was one of them - together with "functional group" and "functional species" strategies. Jeltsch et al. (2008) also pointed to the fact that it was not possible to easily separate these strategies from each other. In any case, none of the modelling papers explicitly use the classification by Jeltsch et al. (2008). If researchers did so, it would certainly clarify terminology.

In our review, we distinguish between models applying functional types and functional traits, following the shift in the theoretical literature from describing vegetation types to describing vegetation function (Moore and Noble, 1990; Webb et al., 2010) reflected in the development of DGVMs (Van Bodegom et al., 2012). The inconsistent use of terminology biases systematic reviews that use key words in search engines. For example, some papers state that they implement a novel trait-based modelling approach although in practice they use functional types. Other papers clearly consider well-developed trait-based models but are not found using that key word (e.g. Seifan et al., 2012). Some studies develop crucial theoretical frameworks or methods that are probably useful for future model development and validation but do not themselves use any model. They advocate, for example, incorporating other organisms in plant trait-based models (Treseder, 2016), including community trait distributions to overcome the challenge of estimating single traits (Edwards, 2016), considering intraspecific variability (Burton et al., 2017), or using remotely sensed data to parameterize trait-based models (McDowell and $\mathrm{Xu}, 2017)$. Nevertheless, not all facets of trait-based modelling seem to be sufficiently well known in all fields of ecology to warrant correct attribution of a study to this method. Thus, unambiguous terminology requires more attention in the future. Adhering to a consistent terminology will also simplify the exchange of trait-based concepts between different disciplines. 
Exchange of ideas on implementing trait-based models occurred between the fields of vegetation ecology, marine ecology, limnology, animal ecology and microbial ecology. This exchange already started with the first trait-based approaches from plant functional ecology (Lavorel \& Garnier, 2002; Wright et al., 2004) being adopted by animal studies of bats (Lentini et al., 2015) and birds (Scherer et al., 2016). In ecological modelling, ideas were transferred from phytoplankton research (Follows et al., 2007) to a litter decomposition model (Allison, 2012). We encourage the expansion of such exchange of trait-based modelling approaches between disciplines. These exchanges are likely to be most promising for cases where different organisms have similar functions in their communities.

Trait-based models have been implemented for answering a number of ecological research questions from basic and applied ecology. Basic ecological questions that were addressed with traitbased models included goals such as identifying which mechanisms drive plant growth, how populations develop over time and space, how communities assemble and biodiversity can be explained, as well as which factors influence community dynamics. Applied trait-based modelling studies investigated biological invasion conditions and consequences, responses of ecosystems to climate and land-use change, conservation and management planning, as well as the evaluation of ecosystem services. We see potential for the reinforcement of trait-based modelling approaches in areas such as the assessment of ecosystem services, invasion prediction and prevention, biodiversity studies, connection to demographic approaches (Salguero-Gómez, Violle, Gimenez, \& Childs, 2018) and, especially, the prediction of community and ecosystem responses under climate and land-use changes.

Many model types were employed to implement trait-based approaches. The greatest proportion was equation-based models. The next greatest proportion was that of statistical models that describe patterns and demonstrate correlations between, for example, functional traits and environmental filters. The combination of trait-based approaches with process-based modelling, as one subcategory of equation-based modelling, is particularly interesting because the detailed representation of physiological processes in process-based models may not at first be compatible with the aggregated approach of trait-based models. However, once united in a model, it is possibly just these different perspectives on a study system that, by complementing each other, will overcome the 
limitations from which the constituent approaches suffer when used in isolation (Scheiter and Higgins, 2009; Ali et al., 2015; Holzwarth et al., 2015). Combined process- and trait-based models are also able to capture a broader range of scales than each approach alone. Trait-based models are challenging to implement at extreme scales because the trait concept aggregates information too much for very finescale models and too little for very broad-scale models. However, traits are successfully integrated into process-based models that implement plant physiology at fine scales and into DGVMs and earth system models at global scales.

Individual-based approaches (Grimm and Railsback, 2005; DeAngelis and Mooij, 2005) are well suited to implement trait-based models, because they can capture variation of trait values at the individual level (May et al., 2009; Scheiter et al., 2013; Weiss et al., 2014; Pontarp and Wiens, 2017). Despite of the apparent similarity between trait-based modelling and individual-based modelling, when considered in isolation, there are differences between them in the main entities of the models (traits versus individuals) and in the way interactions are represented (Fig. 1.4). Nevertheless, traitbased models can easily accommodate individual-level variation, and in individual-based models interactions are usually mediated by traits (Fig. 1.4). Thus, it is straightforward to combine these two approaches, yielding several advantages: It is possible to link traits directly to environmental conditions, so that combined individual- and trait-based models are considered to be an adequate tool for investigating community responses to environmental gradients (McGill et al., 2006; Webb et al., 2010). Moreover, combined individual- and trait-based models are able to offer sufficient flexibility to simplify the description of individuals, to capture plant-plant interactions at the individual level and thereby explain local community-level phenomena (Jeltsch et al., 2008), and to facilitate model parameterization based on trait data that are becoming increasingly available through databases (Weiss et al., 2014; Grimm and Berger, 2016). Trait databases will become an even richer source for traitbased modelling once they expand their current focus on plants to other organisms and start collecting and offering information on abiotic and biotic interactions as well as intraspecific trait variation (Funk et al., 2016). 
A. Trait-based modelling:

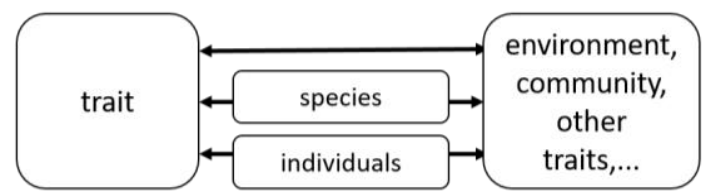

B. Individual-based modelling:

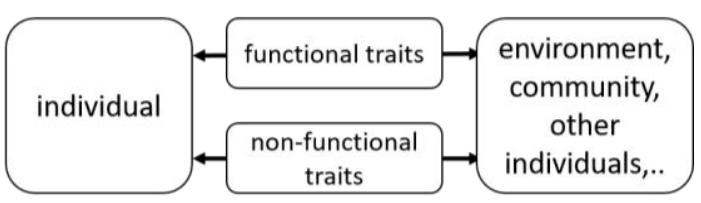

Figure 1.4. Trait-based modelling differs from individual-based modelling in the main entities of the models (traits or individuals, respectively) and in the way interactions are represented (arrows). A. In trait-based modelling, interactions between traits and other traits, populations, communities or the environment can be of three types: 1. direct, e.g. biomass influences population growth rate; 2 . mediated by species, if interspecific trait variability is considered; or 3. mediated by individuals, if intraspecific trait variability is considered. B. In individual-based modelling, interactions between individuals and other individuals, populations, communities or the environment are always mediated by traits. Functional traits are linked to organismal functions such as growth, reproduction and survival. Non-functional traits are not directly linked to such functions and include, for example, $\mathrm{x}$-y-coordinates of individuals. Note that in both cases (A. and B.), arrows represent interactions and any process that is related to the respective interaction, such as individual survival, growth, or reproduction.

We conclude that although trait-based modelling approaches have rapidly increased in ecology over the past twenty years, the potential advantages of the method have not yet been fully exploited. Key terms should be uniquely defined and the main concepts of the theoretical framework should be unambiguously clarified. We recommend developing and applying trait-based models to study community structure and dynamics and to attempt predicting the direction and intensity of community changes under global climate and land-use change. The complexity of such community-level studies is outweighed by the usually lower parameterization effort and more general model outcomes of traitbased modelling approaches. We recommend combining individual-based with trait-based approaches more frequently to benefit from the enhanced flexibility. Moreover, trait-based modelling enables the capturing of the feedback from communities to the environment, as long as the model includes the effects as well as the responses of ecosystems and traits. Trait-based modelling is therefore able to become an important contributor to a comprehensive understanding of community structure and dynamics under global change.

\section{Acknowledgements}

This research was funded by "Niedersächsisches Vorab" (MWK ZN3155) and Volkswagen Foundation. 


\title{
Chapter 2. Combining trait- and individual-based modelling to understand desert plant com- munity dynamics
}

This chapter was published as: Zakharova, L., Meyer, K. M., \& Seifan, M. (2020). Combining traitand individual-based modelling to understand desert plant community dynamics. Ecological Modelling, 434, 109260. https://doi.org/10.1016/j.ecolmodel.2020.109260.

Authors' contribution: K.M.M. and L.Z. conceived of the presented idea. L.Z., K.M.M and M.S. designed the method. L.Z. developed the model and performed the analysis with a large input from K.M.M. 's side. L.Z. led the writing of the manuscript with extensive input from K.M.M. and M.S. Model code is available at: https://github.com/lazakharova/PhDThesis

\begin{abstract}
Understanding the mechanisms driving community dynamics helps us to make reliable predictions about communities' response to environmental change. Studying desert plant communities is particularly challenging because of strong intra- and interannual fluctuations in precipitation. Models rise to this challenge by providing an arena for systematic evaluation of the parameter space in virtual experiments. We applied a trait- and individual-based model to explore how community dynamics arise from the plant traits and interactions of plants among themselves and with their environment. The model is based on data from annual plant communities in the Negev Desert dominated by the True Rose of Jericho (Anastatica hierochuntica). We showed that functional traits that are involved in plant-plant interactions are equally important for community dynamics as traits promoting tolerance to abiotic stress. The sensitivity analysis of the model highlights relative growth rate, maximum biomass, the amount of time in dormancy and germination probability as the most important traits for community dynamics. The model reflects the particular importance of environmental factors such as precipitation and soil water availability based on topography for community dynamics. Our model benefits from the ability of individual-based models to capture plant-plant interactions and derive community properties from individual characteristics and from the feature of trait-based approaches to link traits
\end{abstract}


to organismal functions. Our study demonstrates the advantages of the combined use of trait- and individual-based models for investigating community drivers in changing extreme environments.

\subsection{Introduction}

The fate of desert plant communities is unclear in the face of climate change (IPCC, 2018). An extensive understanding the mechanisms driving plant community dynamics is important to predict community responses to climate change. However, the drivers of community dynamics under arid environmental conditions are not well known. This uncertainty is partly due to strong intra- and interannual fluctuations in water supply (Chesson et al., 2004), which are difficult to capture with relatively short-term empirical investigations. According to future climate-change predictions, interannual variability in precipitation will only increase, which may generate extreme weather conditions (IPCC, 2018).These alterations in precipitation patterns may cause loss of desert vegetation, including annual plants, which are a major component of the vegetation community in these regions (Gutterman, 2000). Besides water availability (Loik et al., 2004) and its pulsed nature (Chesson et al., 2004), plant growth and survival are affected by extreme temperatures, temperature fluctuation (Gutterman, 2000) and soil salinity (Gutterman, 1997). Deserts are usually considered to be particularly vulnerable to climate change as their inhabitants may already grow at their physiological limits (Ward, 2009; Vale and Brito, 2015). However, some studies claim that the communities of annual plants might be not as vulnerable as is commonly assumed (e.g. Salguero-Gómez et al., 2012). To support the empirical investigation of the possible vulnerability of annual plant communities, we present a simulation model that uses a trait- and individual-based modelling approach. This approach provides the necessary tools to explore how community dynamics arise from individual plant traits and plant interactions in an everfluctuating environment.

Ecological modelling is highly suitable to explain the mechanisms underlying plant community dynamics in deserts. An obvious advantage of modelling tools over empirical approaches is the opportunity to test different hypotheses without an intrusion into real ecosystems (Meyer et al., 2009). For instance, ecohydrological conditions in deserts are studied in a variety of models addressing run- 
off and run-on processes (Howes and Abrahams, 2003), dew effects (Gerlein-Safdi et al., 2018), basic flow processes (Dijkema et al., 2018), effect of fog on soil moisture dynamics (Li et al., 2018), extraction of groundwater through roots (Wang et al., 2018) and groundwater flow in two dimensions (Sun et al., 2018). Ecosystem processes including water, carbon and nitrogen fluxes are also represented in the desert modelling literature (Chen and Reynolds, 1997; Reynolds et al., 2004). Considering the vulnerability of desert vegetation to climatic and anthropogenic change, it is surprising that most of the influential models on the topic are more than ten years old. Examples include models considering dispersal and germination strategies of annuals (Venable and Lawlor, 1980), calculating optimal biomass allocation (King and Roughgarden, 1982) or modelling shrubs (McAuliffe, 1988; James et al., 2005). Although global vegetation models target desert vegetation as a special case (Reick et al., 2013), we are not aware of any models that address community structure and dynamics of desert annuals.

Trait-based approaches provide a holistic understanding of the processes driving desert plant communities. These approaches link individual characteristics of organisms with their influence on organism performance and community functions (Violle et al., 2007). Desert plant strategies to cope with abiotic and biotic stress are linked to a set of plant functional traits. Such a trait set connects individual performance with the ability of an organism to tolerate changes in its environment and endure competition from its neighbours. Based on this, functional traits might be divided into two groups representing traits that mediate plant-plant interactions versus traits that help coping with harsh abiotic conditions. These two groups of traits represent the opposing sides of the growth-survival trade-off (Grime, 1977). These interactions with the environment and with neighbours give rise to community structure and dynamics at the local scale (Lortie et al., 2004). Thus, due to the link between traits and interactions, trait-based approaches can help to explain the processes underlying community structure and dynamics. Such mechanistic explanations are the basis of predictions of the fate of desert plant communities (Webb et al., 2010).

Combining modelling and trait-based approaches provides a better understanding of the processes that drive desert plant communities and define their response to environmental changes (May et al., 2009; Weiss et al., 2014). Trait-based models may assist in revealing the mutual interaction between interspecific and intraspecific plant trait variability and changes in the environment and in the 
community (Auger and Shipley, 2013; Kazakou et al., 2014; Grassein et al., 2014; Jeffers et al., 2015). Individual-based modelling is a particularly promising method in combination with trait-based approaches (Zakharova et al., 2019). Individual-based modelling follows a bottom-up approach, where population- or community-level dynamics are derived from interactions between individual organisms and their environment (Grimm and Railsback, 2005) and capturing plant-plant interactions at the individual level to explain local community phenomena (Jeltsch et al., 2008). Trait-based approaches can bridge the gap between the specific phenotypic characteristics of individuals, species performance in particular habitats, population dynamics, and community dynamics including species interactions (Salguero-Gómez et al., 2018; Zakharova et al., 2019). Thus, combining trait-based modelling with individual-based modelling may assist in overcoming limitations of computing power and lack of data as well as enrich models by including functional links between individual organisms and higher levels of organization.

We aimed at identifying the plant traits and environmental factors that drive the dynamics of a community of desert annuals, particularly concerning traits that mediate plant-plant interactions versus traits that help plants to cope with harsh abiotic conditions. Traits that mediate plant-plant interactions include, for example, maximum biomass and relative growth rate. Traits related to coping with harsh conditions include species-specific dispersal parameters, the species-specific number of seeds, and the probabilities to survive and germinate. To tackle our research question, we developed a trait- and individual-based simulation model of plant communities of annuals dominated by Anastatica hierochunti$c a$ in the Negev desert in Israel. Each species in this model was represented as a set of plant functional traits. For now, we focused on two-species interactions to represent two contrasting dispersal strategies. We considered 'escape' and 'protection' (Gutterman, 2000) seed dispersal strategies as important functional traits of desert annual plants ensuring their survival in the desert environment. Species with an 'escape' strategy produce large numbers of small seeds, which are dispersed unconditionally. Species with a 'protection' strategy have developed long-term seed banks with seeds that are kept on the mother plants and are only dispersed in portions by rain (Gutterman, 2000). 


\subsection{Methods}

\subsubsection{Research site}

Model structure, parameterization, and validation are based on an annual plant community in the southern part of the Negev Desert, Israel. This part of the desert is characterized by mean annual precipitation of less than $80 \mathrm{~mm}$, allocated from September to May ("The Meteorologic Service Database, Israel Government Portal," 2020). Soils in this region are reg soil and coarse desert alluvium, which have properties of loess soils regarding wilting point and field capacity (Dan et al., 1976). As a proofof-principle that the model simulates long-terms patterns similar to those observed in nature, we parameterized the model with environmental parameters and plant functional traits from a field site in the Negev. For this, we focused on a two-species community based on two of the most dominant annual plant species at the Meishar site $\left(30^{\circ} 24^{\prime} 48.3^{\prime \prime} \mathrm{N} 34^{\circ} 56^{\prime} 37.9^{\prime \prime E}\right)$. For this, we considered the plant functional traits of Anastatica hierochuntica L. (Brassicaceae) and Malva parviflora L. (Malvaceae) (Appendix. Tab. A3.1).

\subsubsection{Developing a trait- and individual-based model}

We developed the individual-based ATID-model (ㅁnastatica Trait-Based and Individual-based Desert model; atid - a Hebrew word meaning future) to investigate, which environmental factors and plant

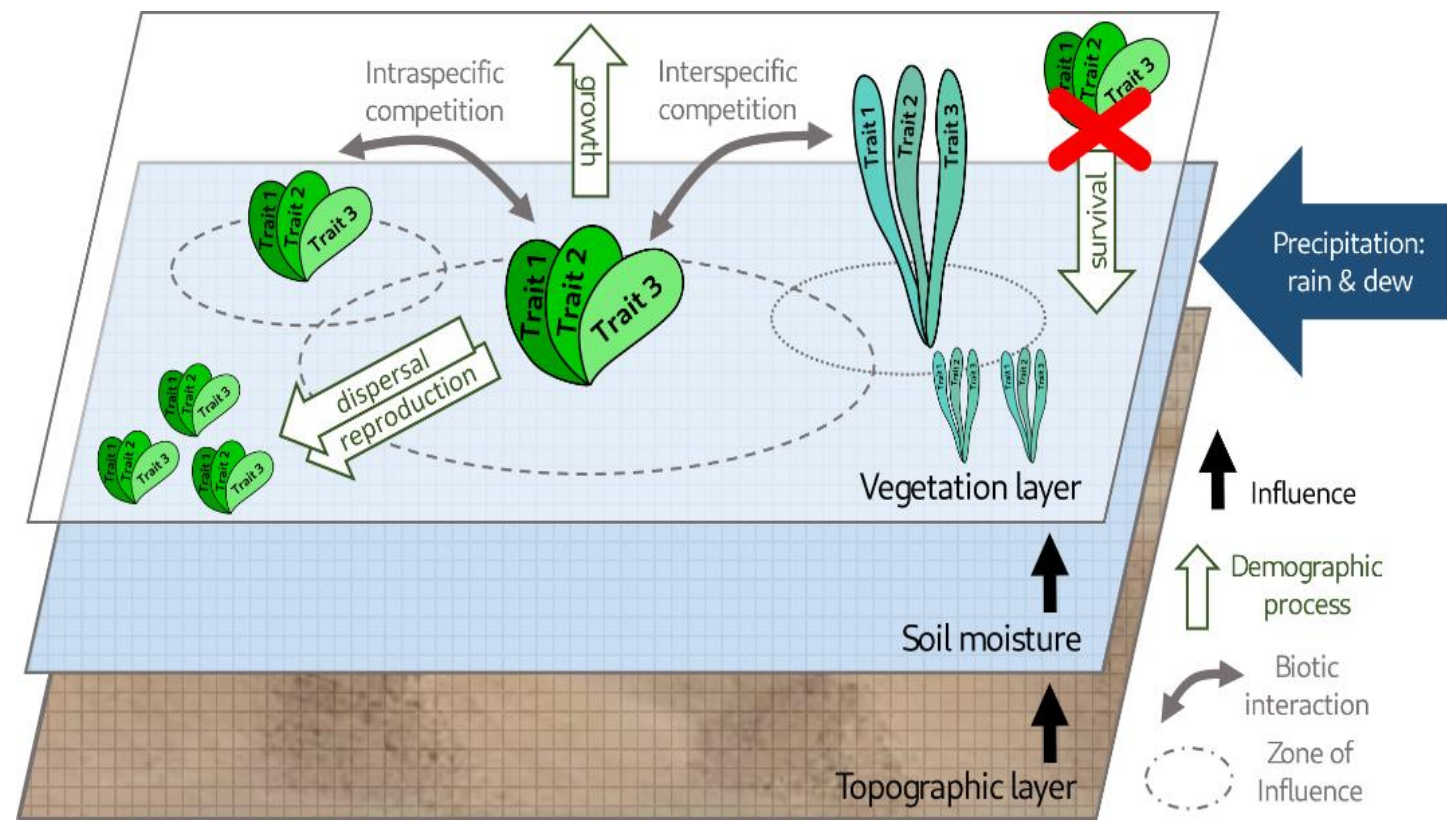

Figure 2.1. Conceptual scheme of the ATID-model. 
functional traits are important for plant performance and community dynamics in deserts (Fig. 2.1).

We implemented the model in NetLogo (Wilensky, 1999), version 6.0.2 (Appendix. Model code: https://github.com/lazakharova/PhDThesis). We combined trait-based (Violle et al., 2007) and individual-based modelling approaches (Grimm and Railsback, 2005). In the following, we describe our model according to the Overview, Design concepts, Details (ODD) protocol for describing individual- and agent-based models (Grimm et al., 2006).

\section{Overview, Design concepts, and Details}

\section{Purpose}

The aim of the ATID-model is to assess the relative importance of environmental factors and traits driving biotic versus abiotic interactions, and how these factors and traits affect long-term community dynamics.

2. Entities, state variables, and scales

Agents/individuals.

Agents are adult individual plants and seeds of two generic species that are inspired by A. hierochuntica and M. parviflora (generic species 'Anastatica' and 'Malva'). These two desert annuals are characterized by two contrasting dispersal strategies: 'protection' and 'escape' strategies (Gutterman, 2000). The model has an in-built opportunity to be extended by an unlimited number of other species with their species-specific functional trait combination, depending on the plant community that the model represents. Each species in this model is represented as a set of plant functional traits related to plantplant interactions and to coping with abiotic stress (Appendix. Tab. A2.1, Tab. A2.2).

Spatial units.

The simulated surface of the model is made up of cells representing arid sites with different topography and soil water availability with a grain of $1 \mathrm{~m}$ by $1 \mathrm{~m}$. 


\section{Environment.}

The environment is simulated as a daily updated soil water availability that depends on two types of precipitation, rainfall and dew input, during the vegetation growth season. The model has embedded rainfall and topography generators.

Temporal scale.

The time step of the model during the simulated vegetation growth season is one day. This time step is small enough to consider daily variation in precipitation and, simultaneously, the main physiological processes involved in growth, survival and reproduction of plants. Outside of the vegetation growth season, there are no daily updates of the model variables. This reflects environmental conditions in the Negev desert, where annual plants grow exclusively during winter, the only wet season in the region. The vegetation growth season is restricted by precipitation availability. Time extent of a model simulation can be set depending on the research aims, but we recommend at least ten years. A ten-year period allows observing relevant fluctuations in environmental factors that may affect the performance of annual plants. The time extent can be prolonged if required.

Spatial scale.

The size of one grid cell is $1 \mathrm{~m}$ by $1 \mathrm{~m}$, corresponding to the scale of field measurements of the environmental factors and vegetation parameters. The spatial extent covers an area of 100 by $50 \mathrm{~m}$ to capture the most important processes at the level of an annual plant community. This extent also reflects the size of the experimental field at the Meishar site used for proof-of-principle-simulations.

\section{Process overview and scheduling}

During each time step, the two main parts of the model, Vegetation and Environment, are executed (Appendix. Fig. A2.1). The simulation loop continues until the specified time extent is reached or no adult plant or seed agents exist anymore.

The Vegetation part consists of several submodels involving seed and adult plant agents. 
Processes at the level of seeds:

Seed germination (Germination submodel) transforms seeds into adult plants if there is enough soil water availability. Seed dispersal (Dispersal submodel) distributes seeds. Seed mortality represents the loss of seeds caused by processes such as disease, granivory, desiccation or other causes of natural mortality. It is incorporated in the Germination submodel as an integrative part of germination probability.

Processes at the level of adult plants:

Adult plants experience natural mortality, inter- and intraspecific competition, growth, ageing and reproduction (Fig. 2.2). Natural mortality is the probability of dying because of any natural mortality factors other than direct competition, e.g. disturbance by wild animal activity (Adult mortality submodel). Competition occurs via the zone of influence (Competition submodel). Growth is represented by an increase in biomass (Growth submodel), based on the share of resources that each adult plant gets in the Competition submodel. Ageing is applied for both seeds and adult plants (Ageing submodel)

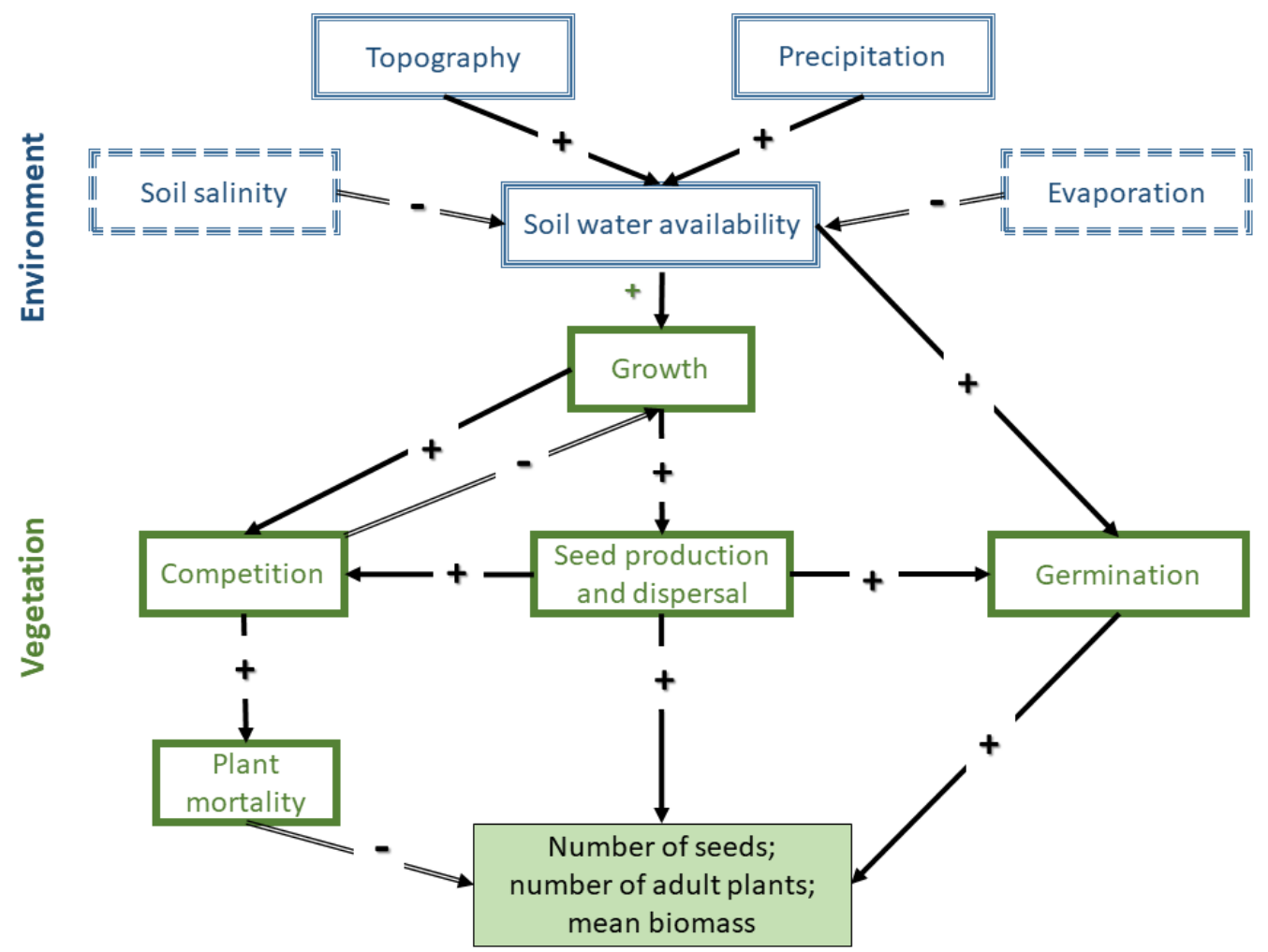

Figure 2.2. Causal diagram of the processes at the core of the ATID-model. The blue boxes with the triple frame show environmental processes; the green boxes with the simple frame show vegetation processes. The boxes with dashed border: salinity and evaporation are not explicitly included in the current model version, but should be explored at greater detail in future model versions. The simple arrows with "+" show the positive influence of the process at the beginning of the arrow on the process at the end of it. The double arrows with "-” reflect, respectively, negative influences. The filled box is the model output. 
because age is relevant for some submodels such as Reproduction and Germination. Reproduction leads to the production of seeds under certain conditions (Reproduction submodel).

Within the Environment part, during each time step that is part of the vegetation growth season, soil water availability is updated for each cell depending on its topography and precipitation (Wateravailability submodel). The model considers topography as the most important environmental factor (Fig. 2.2). This assumption is made based on the nature of the soil in the region that causes a large fraction of the rainfall to move at the local scale from the highest sites to the lowest. All other environmental factors in the model depend on topography. Refined soil water availability and salinity will be incorporated in future model extensions reflecting the progress of ongoing data collections.

\section{Design concepts}

The model adopts a trait-based approach, which provides a link between individual functional traits and properties of populations and communities (Violle et al., 2007). Plant functional traits also reflect strategies adopted by individual plants in response to biotic and abiotic stress. The competition mechanism implemented in the model is based on zones-of-influence, which are circular biomass-depended zones around each plant (Berger et al., 2008).

\section{Emergence.}

Heterogeneity of environmental settings together with the variability of plant functional traits determine emerging plant-plant interactions as well as the patterns of the spatial distribution of adult plants and seeds, population sizes of different species and response trait distributions, e.g. size distributions, at various temporal and spatial scales.

\section{Adaptation.}

In the current version of the model, no adaptive processes are implemented.

Sensing.

Plants and seeds sense soil water. They indirectly sense other plants in the cells within their zone-ofinfluence through shared water resources. 


\section{Interaction.}

Plants interact with their environment by taking up water depending on soil water availability. Plants interact indirectly with other plants during the competition for resources via zones-of-influence.

\section{Stochasticity.}

Stochasticity is directly embedded in most model processes, namely in the construction of the landscape topography, in the temporal distribution of the rain, in seed dispersal, in germination, in growth and in natural mortality. Landscape topography is created by drawing differences in heights of neighbouring cells from a normal distribution. Each year, the number of rain days within the vegetation growth season is drawn from a uniform distribution. Seed dispersal includes two stochastic components, one each regarding direction and distance. The direction is uniformly distributed, while dispersal distances are drawn at random from a Weibull-shaped distribution with species-specific parameters. Both seed germination and plant growth are modelled as probabilities that seeds get water to germinate and plants get water to grow, respectively. This location-specific probability is a function of rainfall, dew, and topography. Natural mortality is modelled as a constant, species-specific probability. In addition to these stochastic processes, depending on the chosen model scenario, the initial distribution of adult plant individuals and seeds in space may also be random

\section{Observation.}

Table 2.1. Potential output values of the model with the units of measurement and level of observation, which can be obtained in the model simulations. These outputs are aggregated data based on individual variables, such as biomass and spatial coordinates, as well as the number of individuals. (The individual level is addressed in the model (e.g. as a carrier of traits), but is not considered as output level in this model version).

\begin{tabular}{ll}
\hline Output (unit) & Level of observation \\
\hline Abundance of species & Population \\
Population density $\left(\right.$ Plant $\left./ \mathrm{m}^{2}\right)$ & Population \\
Population mean of produced seeds & Population \\
Population mean biomass $(\mathrm{g})$ & Population \\
Species composition & Community \\
Spatial pattern of species & Community \\
Spatial pattern of traits & Community \\
\hline
\end{tabular}

The following outputs of the model can be potentially observed at different levels such as individual, population and community (Tab. 2.1). The basis for these compound measures is the number of indi- 
viduals, both adult plants and seeds, their spatial coordinates and biomass of adult plants. In this paper, we used all of these measures except for spatial coordinates as reference values for sensitivity analysis.

\section{Initialization}

Model initialization involves setting up a landscape with the cell heights (parameter height) gradually distributed between the lowest and the highest heights above sea level. The procedure starts with assigning the lowest cell (min-height). The height value of its neighbours is a sum of the height of this lowest cell and an increment calculated as a difference between the highest and lowest height divided by the width of the modelled landscape. A standard deviation (sd-height) is added to this calculated height. Based on this initialization, the following topographic objects can be identified: local maxima, local minima, and slopes (Fig. 2.3). Local maxima are 1 by $1 \mathrm{~m}$ cells surrounded by eight cells (8-cellneighbourhood) lying below them in height. Local minima are cells lying below their eight neighbours in height (these microhabitats correspond with "patches" in Hegazy and Kabiel, 2007). Slopes have at least one neighbour lying above them and at least one neighbour lying below them. These topographic objects differ in their properties in terms of water and seed retention.

The soil seed bank is initialized by creating and randomly distributing seeds throughout the landscape.

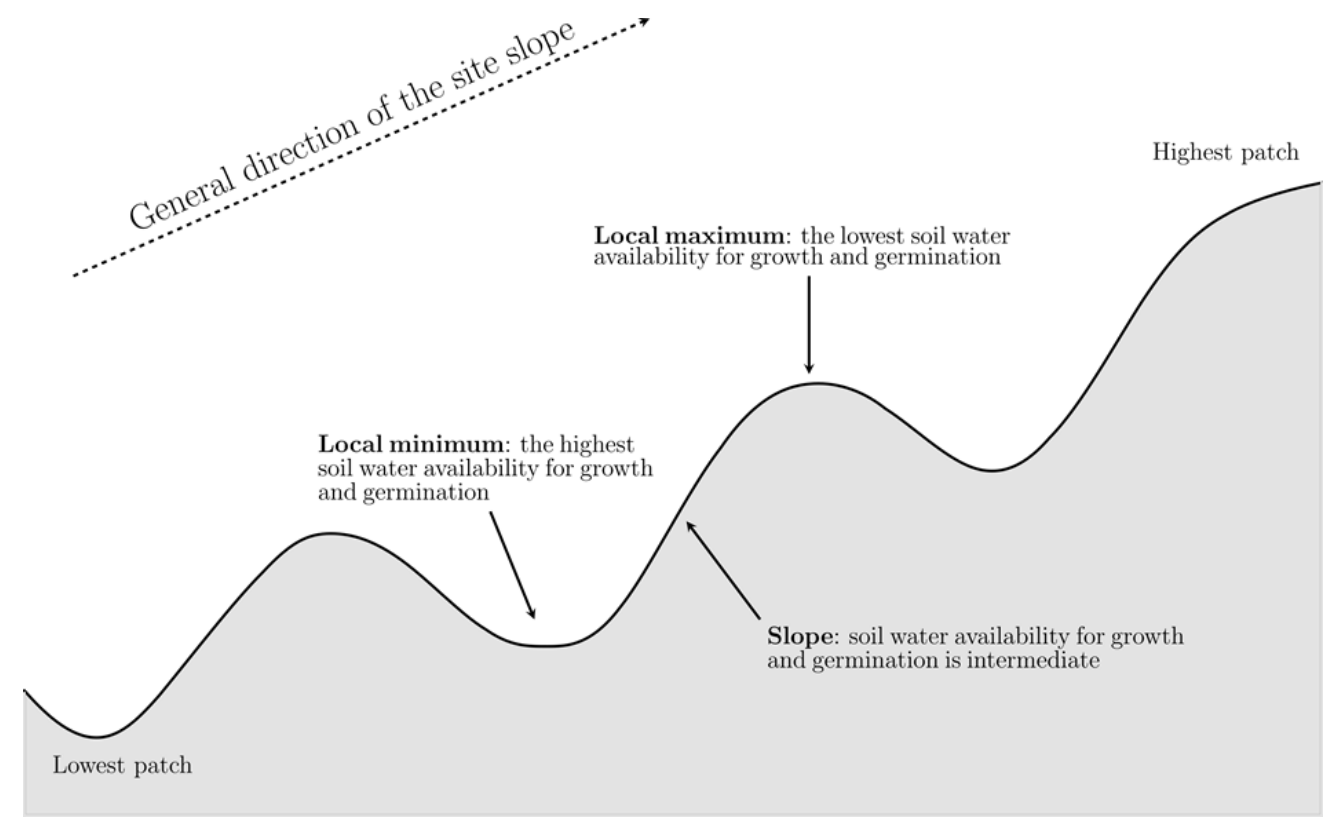

Figure 2.3. Schematic representation of the simulated topographical objects and their influence on the processes of plant growth and seed germination. Note that this figure overemphasizes the steepness of the site slope for visualization reasons, in reality and in the model the overall site slope is relatively shallow (e.g. for the simulation as a proof-of-principle the height difference is $5 \mathrm{~m}$ for the area of 100 by $50 \mathrm{~m}$ in size). 
Seeds are assigned a set of traits. A model simulation starts with the beginning of the first vegetation growth season. Thus, only a specific number of seeds representing each species are created. This approach provides us with as neutral starting conditions as possible, where the influence of the initial conditions on model outputs is minimized.

Depending on topography, the suitability of each cell for seed germination and plant growth is determined (Water-availability submodel, Growth and Germination submodels).

\section{Input data}

Topography and rainfall can be initialized in this model via algorithms (this study) or via input data (not implemented in the analyses presented here). The rainfall data must include the number of rainy days in a year, their sequential order and the overall length of the vegetation growth season. The incorporated rain algorithm is rather simplified due to the absence of suitable rainfall generators for the region generating typical, but not historical, rainfall time series. The topography data must include distribution of heights above sea level (e.g. from digital elevation models). The simulations presented in this paper are based on a topography generator (Initialization) and a rainfall generator (Rain submodel).

\section{Submodels}

Here we explicitly describe the submodels that make up the Environment part and the Vegetation part. We explain theories related to plant physiological processes underlying these submodels and give references to the respective variables and parameter values (Appendix. Tab. A2.1, Tab. A2.2).

\section{Environment part.}

Rain submodel 
Rainy days stimulate plant growth and seed germination through the increase of soil water availability (Water-availability submodel) and initiate dispersal according to the 'protection' strategy (Appendix. Fig. A2.1, Dispersal submodel).

This submodel generates a list of rainy days annually, where the number of the list items is the number of rainy days in a certain year, and the list items themselves are the sequential numbers of the rainy days in the vegetation growth season. The potential number of rainy days in the season is predetermined as well as the approximate duration of the season (Appendix. Tab. A2.1). The distance between the first and the last simulated rain events determines the actual duration of the vegetation growth season in the simulated year. Each simulated year starts with the vegetation growth season. The generated list of the rainy days serves as an input to the model. On rainy days, soil water availability in the cells is updated in accordance with their topographic characteristics (Initialization).

\section{Water-availability submodel}

The Water-availability submodel checks if there is any precipitation, either in the form of rain (Rain submodel) or in the form of dew, updating soil water availability daily during the vegetation growth season. Soil water availability ( $p$-water-availability) is a cell property in the model and depends only on the presence of precipitation and the topographical properties of the cell. Soil water availability is expressed as a probability for plants on this cell to take up enough water for growth (or germination in the case of seeds), and it does not depend on the species identity or age of the plant. There are three soil water availability values: a local minimum, a slope, and a local maximum. These values are assigned to the topographical objects so that a local minimum has the greatest value and a local maximum has the smallest value. This dependency of soil water availability on topography in the model represents the effects of local water run-off from a higher site to lower neighbouring sites and higher evaporation at higher sites (Daws et al., 2002; Hegazy and Kabiel, 2007). In reality, the amplitude of differences in soil water availability between topographical objects depends on soil properties, such as soil texture, which define water holding capacity and infiltration rate (Kramer, 2019). Thus, soil water availability in the model is a derivative of the soil properties, although these soil properties are not modelled explicitly in this model version. 
Dew, as an additional source of water, is particularly important under water constraints in the desert (Hill et al., 2015). Its impact is increasing with the elevation gradient in the Negev (Kidron, 1999). To study possible dew effects on plant growth and survival, we included a mechanism in the model to consider the possible input of dew throughout the vegetation growth season (with additional days before and after this season - dew-days-before and dew-days-after, respectively) and accordingly update soil water availability beyond the rain events within the vegetation growth season. Dew presence equally affects the soil water availability of all the topographic objects. Possible elevation effects on dew are negligible within our research area.

\section{Vegetation part.}

\section{Dispersal submodel}

In this submodel, seeds created in the Reproduction submodel are dispersed depending on a speciesspecific dispersal strategy: 'escape' strategy or 'protection' strategy (Appendix. Fig. A2.1), which, in the simulated plant community of this model version, correspond to the dispersal strategies of M. parviflora and A. hierochuntica, respectively (Gutterman and Ginott, 1994). In general, seeds are only dispersed if they reach the dispersal age (s-disp-age), i.e. if they are mature enough to leave the parental plant.

The 'escape' strategy represents an unspecific mechanism of seed dispersal. The direction of seed dispersal is drawn at random between 0 and 360 degrees, and distance calculation is based on a Weibullshaped dispersal kernel. Parameters $\beta$ (s-disp-shape) and $\delta$ (s-disp-scale) (Appendix. Tab. A2.1) are species-specific (Paradis et al., 2002) and are involved in the calculation of the distance (s-dispdistance) over which seeds are dispersed from a maternal plant, following the cumulative density function $f$ :

$$
f=1-\exp \left[-(d / \delta)^{\beta}\right](\text { Eq. 1), }
$$

after rearrangement of (Eq 1):

$d=-\delta \cdot \ln (1-f)^{1 / \beta}($ Eq. 2$)$,

where $\mathrm{d}$ is a distance (Paradis et al., 2002). 
The 'protection' strategy differs from the 'escape' strategy in an additional protective mechanism. Seeds with the 'protection' strategy are dispersed to other cells only during rain events (Rain submod$e l)$. Such a mechanism increases the probability that the seeds get enough water to germinate. All the following steps, such as setting a direction and distance for dispersal, are equal to the 'escape' strategy. Species-specific parameters $\beta$ and $\delta$ as well as dispersal strategies are considered plant functional traits related to survival in arid environments.

\section{Germination submodel}

The submodel runs only during the simulated vegetation growth season. In the main part of this submodel, seeds, which were already dispersed, germinate and become adult plants (without explicit modelling of the seedling stage). Germination occurs only under the following conditions: soil water availability ( $p$-water-availability) is sufficient for germination, the species-specific probability to germinate is met (s-pr-germ), and seeds have spent the species-specific amount of time in dormancy (sdays-dorm).

An adult plant inherits the functional trait characteristics of the seed from which it emerged. Age of germinated adult plants is set to one. The stage of seedlings is not explicitly modelled, because the seedling stage of annuals lasts only a few days. However, seedling mortality is included by giving adult plants a lower probability of survival until they reach a certain biomass (Adult mortality submodel). This submodel represents the ability of plants to survive in a desert environment. We focus on the amount of time in dormancy and probability to germinate as plant functional traits involved in coping with the harsh environment.

\section{Competition submodel}

This submodel reflects competition of adult plants for space and resources, which are represented in this model version by soil water availability. This submodel determines how space and resources are shared; the actual resource uptake expressed in biomass increase is implemented in the Growth submodel. The submodel runs only during the simulated vegetation growth season. Plants acquire water resources from the neighbouring cells based on their total biomass and asymmetry of competition. The competition mechanism occurs through the zone-of-influence (ZOI) approach (Berger et al., 2008). In 
this submodel, a set of measurements related to the concept of ZOI is calculated, such as the radius of the ZOI and the effective area for resource uptake.

The ZOI of a plant is calculated as a circular area with a radius reflecting its potential resource use. This area can cover more than one cell. The model offers two calculation options for the allometric relationship between the radius of the area of the ZOI and the biomass of a plant:

$$
\text { radius }=\frac{B(i, t)^{3 / 8}}{\sqrt{\pi}}(\text { Eq. } 3) \text {, }
$$

or

$$
\text { radius }=\sqrt{\frac{B(i, t)^{2 / 3}}{\pi}}(\text { Eq. } 4) \text {, }
$$

where $\mathrm{B}$ is plant biomass, $i$ defines an individual in the centre of a given ZOI, and $t$ is a time step, after Lin et al., 2012 (Eq. 3) or after Weiner and Damgaard, 2006 (Eq. 4).

The model offers two alternative implementations of the ZOI approach, Eq. 3, Eq. 6 (Lin et al., 2012; Radny and Meyer, 2018) and Eq. 4, Eq. 7 (Weiner et al., 2001; Weiner and Damgaard, 2006), because it was not possible to choose one over the other purely based on the literature. We suggest that always both implementations are tested to choose the more suitable one for the study at hand based on sensitivity analysis, model calibration, or pattern-oriented modelling (Grimm, 2005).

If a plant has neighbours, i.e. their zones of influence have an overlap, an effective area of these plants is calculated. The effective area is the difference between the area that the plant covers and the area lost to competition with its neighbours (Weiner and Damgaard, 2006). In the calculation of the effective area, the degree of asymmetry (ad-comp-asymmetry or $\theta$ ) of competition is incorporated. This degree of asymmetry is a measure of competition intensity. It reflects how the competing plants share their resources, depending on their biomass relative to the other competitors. The effective area is:

$$
A_{e f f}(i, t)=\Sigma \frac{B i(t)^{\theta}}{\Sigma\left(B j(t)^{\theta}\right)} \cdot R(\text { Eq. 5), }
$$

where $\theta$ is the degree of asymmetry, $B i$ and $B j$ are the biomasses of the interacting plants $i$ and $j, \mathrm{R}$ is the amount of resources shared between individuals (Schwinning and Weiner, 1998). 
If the degree of asymmetry equals zero, resources are shared equally, regardless of plant biomass. If the degree of asymmetry equals one, resources are distributed proportionally to the biomass (perfect size-symmetry) (Schwinning and Weiner, 1998; Weiner and Damgaard, 2006; Lin et al., 2012).

This submodel and the following one reflect the plant-plant interactions in the modelled system. We consider here maximum biomass and relative growth rate as traits involved in these interactions.

\section{Growth submodel}

In the Growth submodel, plants increase their biomass based on the available resources. The submodel runs only during the simulated vegetation growth season and follows the Competition submodel. If the conditions for the Competition submodel are not fulfilled, the Growth submodel is not executed, either. The Growth submodel runs only if the zone-of-influence of an individual is larger than zero and soil water availability ( $p$-water-availability) is larger than a random number.

We implemented two alternatives for the calculation of biomass gain $\frac{d \mathrm{~B}}{d \mathrm{t}}$ as a function of the effective area $\left(A_{\text {eff }}\right)$ described in the Competition submodel:

$$
\begin{aligned}
& \frac{d \mathrm{~B}}{d \mathrm{t}}=r g r \cdot\left(A_{e f f}-\left(\frac{B^{2}}{B_{\max } x^{\frac{4}{3}}}\right)(\text { Eq. 6), }\right. \\
& \text { or } \\
& \frac{d \mathrm{~B}}{d \mathrm{t}}=r g r \cdot A_{e f f} \cdot\left(1-\left(\frac{B}{B_{\max }}\right)^{1 / 4}\right)(\text { Eq. } 7),
\end{aligned}
$$

where $B_{\max }$ is the maximum asymptotic biomass and $\mathrm{rgr}$ is a species-specific relative growth rate after (Weiner and Damgaard, 2006; Radny and Meyer, 2018) (Eq. 6) or after Lin et al., 2012 (Eq. 7). The relative growth rate is constant throughout all stages of the plant development in our model. The model does not consider the distribution of biomass between plant organs such as leaves, stem, and roots. Biomass is equal to all the biomass gained in the process of growth. Plants do not lose biomass because of maintenance and metabolism processes. The biomass gained by a plant during a year is positively correlated with the number of seeds it produces.

The model assumes that plants use all of the water available in the soil during one day according to their share in the competition process. The change of soil water availability due to uptake by plants is 
not explicitly modelled to keep the model simple. Any water left after the uptake is considered lost to evaporation.

Ageing submodel

In the Ageing submodel, the age of both seeds and adult plants is increased daily. The age update is relevant for the submodels Reproduction, Dispersal and Germination. Adult plants cannot reproduce until they reach the age of reproduction (ad-age-repr). In the Dispersal submodel, the dispersal is possible only if seeds reach a specific age (s-disp-age). Seeds also cannot germinate before they have spent a certain period of their life in dormancy (Germination submodel).

\section{Reproduction submodel}

This submodel allows adult plants to produce seeds. If an adult plant has reached the age of reproduction (ad-age-repr) and the biomass of reproduction (ad-biomass-repr), it produces several seeds dependent on the plant biomass (s-number-actual) with a certain seed weight (s-mass). This actual number of seeds is calculated based on a biomass of reproduction (ad-biomass-repr), which is the minimum biomass needed for the production of at least one seed.

Based on the assessed reproduction effort, the number of seeds, which this specific plant can produce at a certain time step, is calculated:

s-number-actual $=$ int (ad-biomass / ad-biomass-repr),

where ad-biomass is the actual biomass of this plant and int is a function, which returns the integer part of the argument.

These seeds receive all functional trait characteristics of their parental adult plant and are dispersed (Dispersal submodel). In the model, an adult plant loses biomass equal to the multiplication result of seed weight and produced seed number:

$$
\text { ad-biomass }=\text { ad-biomass }-s \text {-mass } * \text { s-number-actual }
$$

A plant can reproduce more than once during the vegetation growth season if it again reaches the minimum biomass necessary for the production of at least one seed (ad-biomass-repr). The counter of the 
seeds (count-s-number) controls that the plant does not produce more seeds than a species-specific number.

The reproduction process is a strategy for annuals to survive under harsh environmental conditions, and seed weight, reproduction age and biomass are plant functional traits involved in this process.

\section{Adult mortality submodel}

Adult plants die randomly with a species-specific probability of survival during a year (ad-prob-surv), which reflects the natural mortality caused by processes that are not explicitly modelled (such as damage caused by wild animals or herbivores). If plants have not reached a certain fraction of the maximum biomass (juv-biomass), they are exposed to a lower survival probability (juv-surv). Seed mortality is included in the germination probability. The survival probability of plants is considered as a plant functional trait that provides information about the survival strategies in a desert environment.

\subsubsection{Model analysis}

To assess the relative effects of the model parameters on model output, we conducted a sensitivity analysis (Appendix. Tab. A2.1) with R (R Core Team, 2018; version 3.5.1) using the package "nlrx" (Salecker and Sciaini, 2019). The nlrx package provides efficient tools to set up, run, and analyse NetLogo model simulations in R (Salecker et al., 2019).

The parameter space of the entire model was analysed in a global sensitivity analysis. To efficiently sample the parameter space, we applied the Morris screening procedure (Morris, 1991), where only one parameter out of all model parameters is changed at each step. We used two output indices of the Morris screening procedure to assess the relative influence of parameters: $\mu^{*}$ and $\sigma$. The index $\mu^{*}$ is the mean of the absolute values of the elementary effects and $\sigma$ is the standard deviation of the elementary effects (Iooss and Lemaître, 2015).

For each model parameter, we provided a minimum and a maximum value to the Morrisscreening algorithm according to expert knowledge (Appendix. Tab. A2.1). As the output reference values of the analysis ("metrics" in the nlrx package), we chose mean biomass of adult plants, number 
of adult plants and number of seeds of two generic species with 'escape' ('Malva') and 'protection' ('Anastatica') seed dispersal strategies over the vegetation growth season in the first model year. We chose to run the analysis for one simulated year to keep the processing time short and capture output reference values for those parameter combinations that lead to the death of all seeds and adult plants in the first year. We checked the model output sensitivity to many plant functional traits and environmental parameters (Appendix. Tab. A2.1). The sensitivity analysis was performed under eight scenarios combining the absence or presence of dew with one of two calculations of ZOI and plant growth (Weiner \& Damgaard, 2006 and Lin et al., 2012) and different combinations of two seed dispersal strategies.

The initial number of adult plants and the initial number of seeds were not varied during Morris screening, simulating the beginning of the vegetation growth season.

To address the large numbers of parameters and to minimize processing time, we kept the following parameters fixed: asymmetry of competition, the lowest height in the topography generator and the shape parameter of the Weibull distribution. The asymmetry of competition parameter was set to one as the model considers belowground competition for water as the main limiting resource in deserts (Fonteyn and Mahall, 1978) and belowground competition is considered symmetric (Weiner et al., 1997). We set the lowest height to one, guided by common sense that the minimum height defines the starting point from which all other heights and differences are calculated. We set the shape parameter of the Weibull distribution to one, which corresponds to an exponential-like distribution function (García and Borda-de-Água, 2017). In total, we tested the influence on the model output of 37 parameters (Appendix. Tab. A2.1).

\subsection{Results}

\subsubsection{Model development and validation}

We developed a running model that successfully passed visual plausibility checks. Visual diagnostics (Mayer and Butler, 1993) demonstrated that the model output is similar to informal empirical observa- 
tions with respect to annual vegetation growth peaks, long-term species co-occurrence of two species and interannual variation in mean biomass of a population (Fig. 2.4).
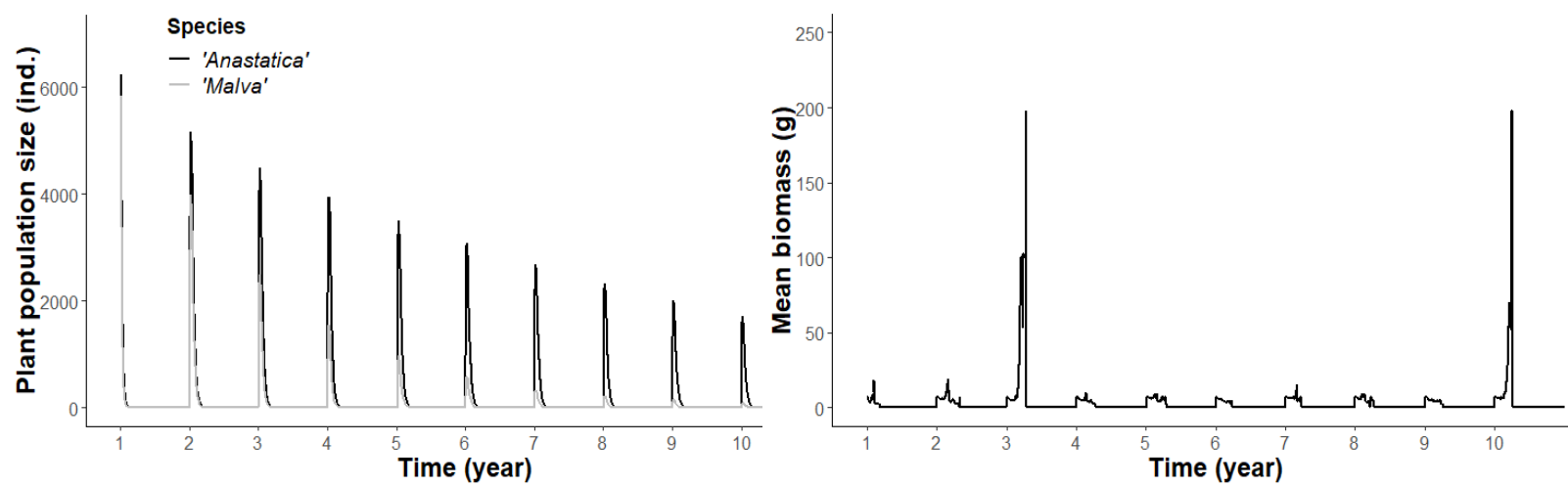

Figure 2.4. 10-year simulation of the ATID model. Left panel: seasonal fluctuations in plant numbers and co-occurrence of two species over time. Right panel: interannual fluctuations in mean biomass of one of the model plants (generic species 'Anastatica'). The source of most plant functional traits are the traits of the species A. hierochuntica ('Anastatica') and $M$. parviflora ('Malva'). Vegetation growth seasons are indicated by non-zero population sizes.

\subsubsection{Results of sensitivity analysis}

We analysed the results of Morris screening for eight scenarios based on the values of $\mu^{*}$ and $\sigma$. In the
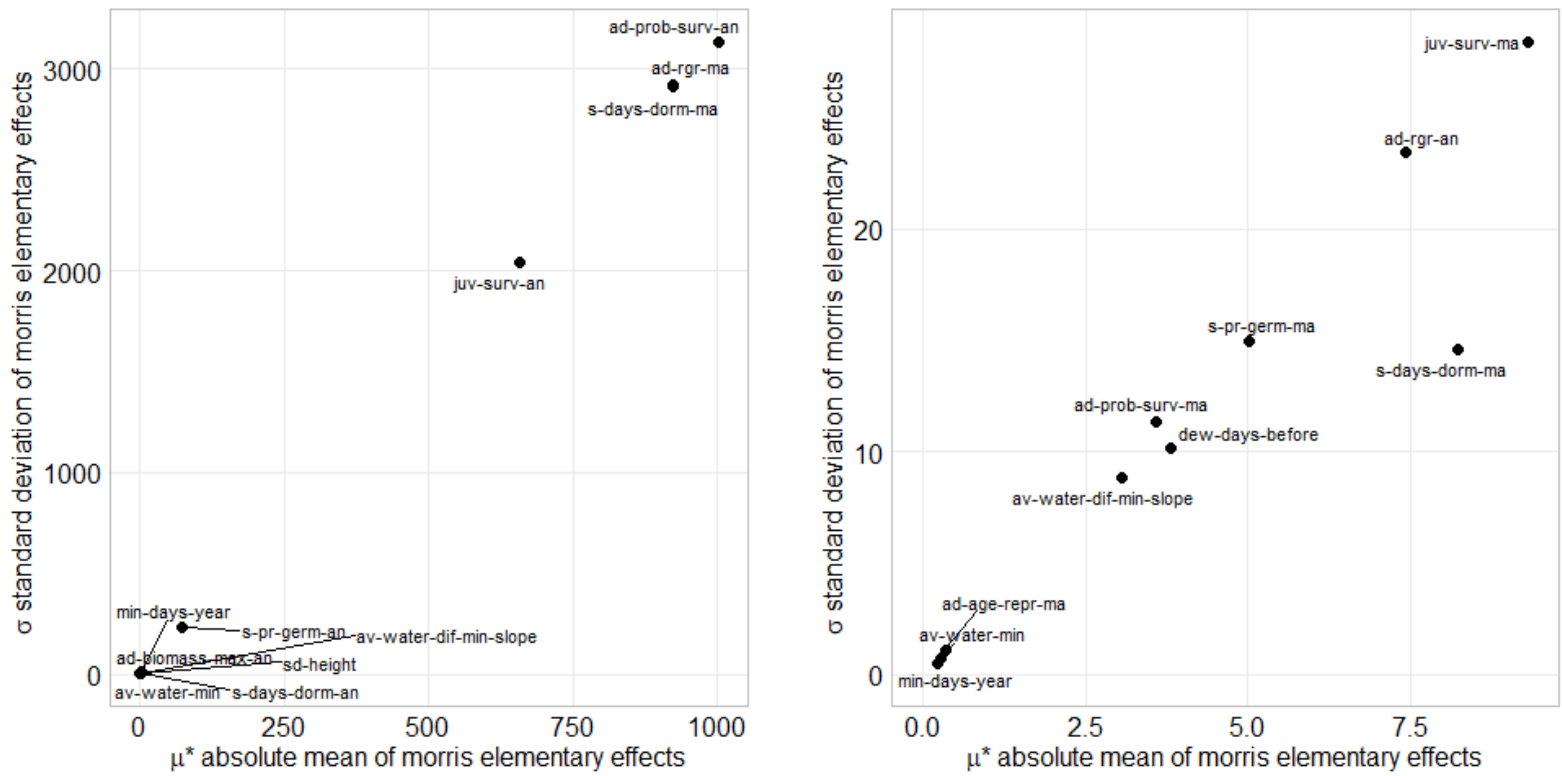

Figure 2.5. Results of the sensitivity analysis for number of adult plants as output metric and for the scenario with ZOIcalculation after Lin et al., 2012 and dew present in the model. Left panel: 'Anastatica', right panel: 'Malva', differing mainly in dispersal strategy. The graphs show the relationship between the absolute mean of the Morris elementary effects $\mu^{*}$ and the corresponding standard deviation of the Morris elementary effects $\sigma$. Larger $\mu^{*}$ means that the corresponding parameter has a larger effect on the output. Larger $\sigma$ means either that the corresponding parameter has a non-linear effect on the output or that this parameter is involved in interactions with other parameters. For clarity of representation, only the parameters that are among either the largest ten values of $\mu^{*}$ or among the largest ten values of $\sigma$ are shown. The parameter suffixes $-a n$ and $-m a$ correspond to 'Anastatica' and 'Malva'. 
following, we consider the influence of the parameters and scenarios on the model output following the order of the corresponding model parts and submodels (Appendix. Fig. A2.1).

\section{Environment part.}

Topographical parameters from model initialization did not have a large influence in any scenario. The difference between the highest and the lowest site affected only mean biomass out of all possible output reference values, and the standard deviation of the smooth slope only influenced the number of adults. The standard deviation of the smooth slope also had a high $\sigma$ value implying either a non-linear effect or interactions with other parameters (Appendix. Fig. A2.2).

Among the environmental parameters of the Rain submodel, the minimal number of rainy days per year and the overall length of a rainy season were highly influential parameters. Both of these parameters influenced largely the number of adult plants and number of seeds (Fig. 2.5; Fig. 2.6). For the scenarios considering the dew effect, the number of days before the first rain, during which dew contributes to water availability in the model, showed a great influence on all output reference values, except for the number of adult plants of the generic species with 'protection' dispersal strategy ('Anastatica').
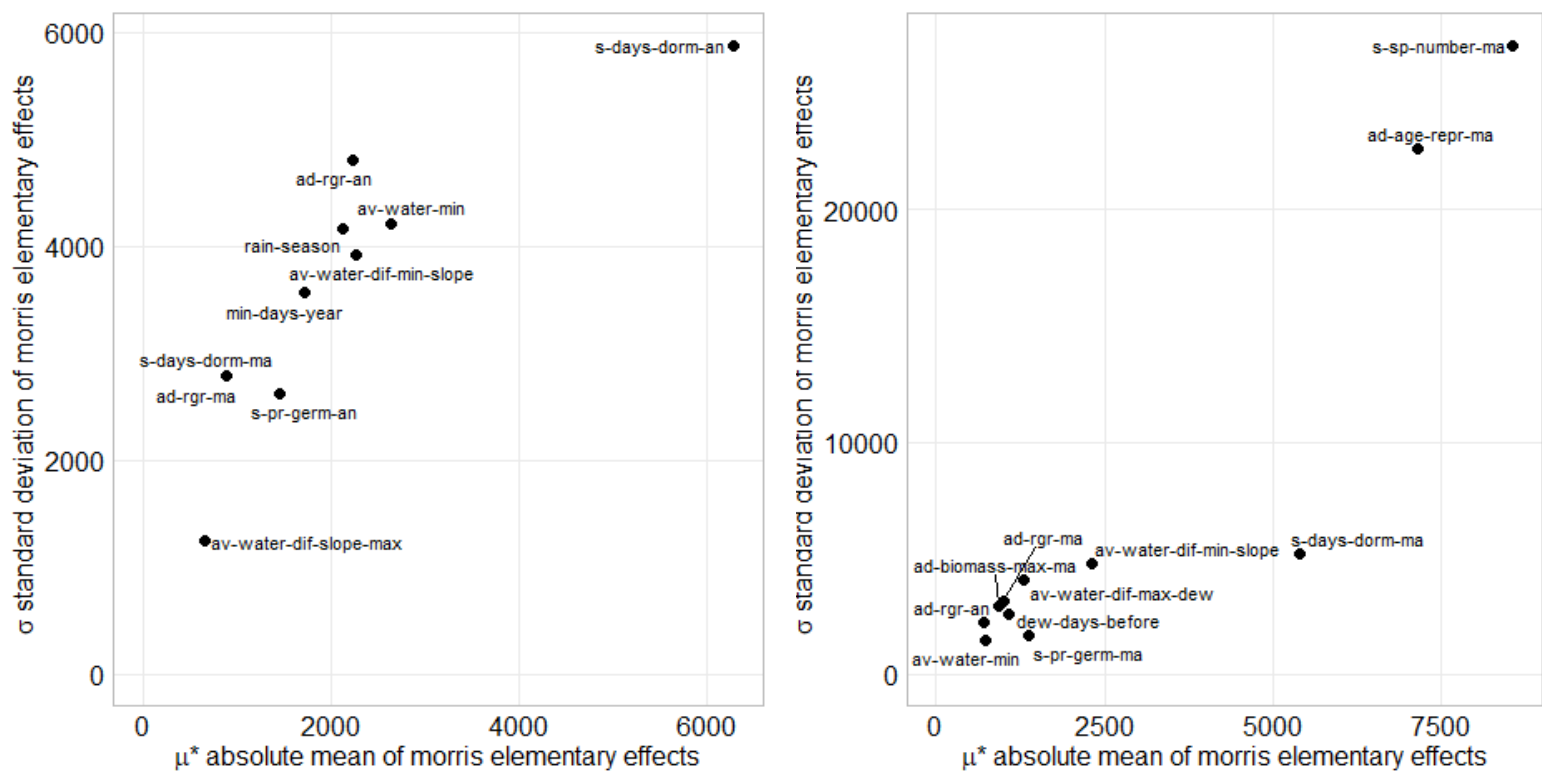

Figure 2.6. Results of the sensitivity analysis for number of seeds as output metric and for the scenario with ZOI-calculation after Lin et al., 2012 and dew present in the model. Left panel: 'Anastatica', right panel: 'Malva', differing mainly in dispersal strategy. The graphs show the relationship between the absolute mean of the Morris elementary effects $\mu$ *and the corresponding standard deviation of the Morris elementary effects $\sigma$. Larger $\mu^{*}$ means that the corresponding parameter has a larger effect on the output. Larger $\sigma$ means either that the corresponding parameter has a non-linear effect on the output or that this parameter is involved in interactions with other parameters. For clarity of representation, only the parameters that are among either the largest ten values of $\mu^{*}$ or among the largest ten values of $\sigma$ are shown. The parameter suffixes - an and - ma correspond to 'Anastatica' and 'Malva'. 
The number of the days after the last rainy days, when dew still can affect plant growth, in contrast, had very little influence on any output reference values, although both of these parameters increase the length of the possible vegetation growth season.

Regarding the parameters of the Water-availability submodel, the model demonstrated high sensitivity to water availability at local minima and to the difference between local minima and slopes for all the scenarios. Both these parameters influenced largely the number of seeds and number of adult plants, but much less biomass outputs. The difference in water availability between slopes and local maxima influenced only the number of seeds. For scenarios with dew effect included, the difference in water availability between local maxima and dew contribution to water availability had a high influence on the seed number (Fig. 2.5; Fig. 2.6).

\section{Vegetation part.}

The parameters of the Dispersal submodel such as the age at dispersal and the scale parameter of the dispersal distribution had no or little influence on model output. Surprisingly, there were no re-
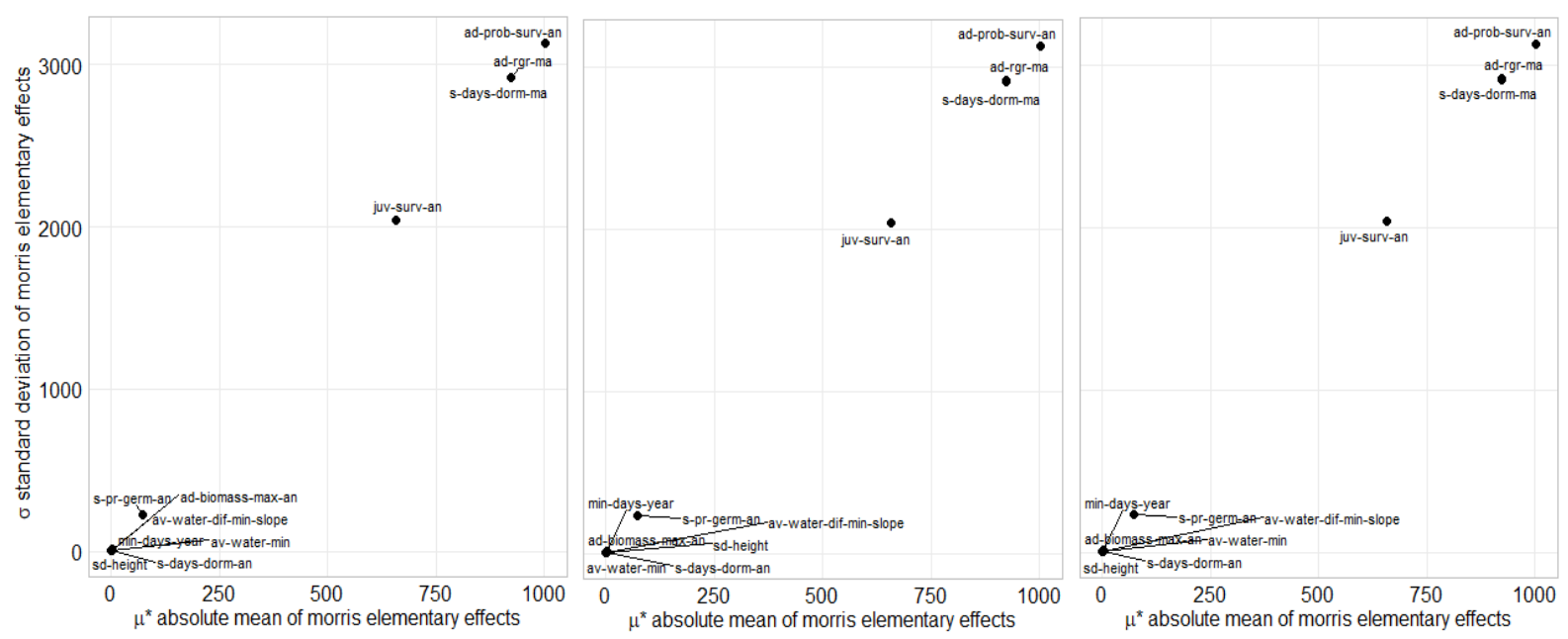

Figure 2.7. Results of the sensitivity analysis for number of adult plants for 'Anastatica' as output metric and for the scenario with ZOI-calculation after Lin et al., 2012 and dew present in the model. First panel: both 'Anastatica' and 'Malva' have 'escape' strategy; second panel: ‘Anastatica' has 'protection' strategy and 'Malva' has 'escape' strategy; third panel: both 'Anastatica' and 'Malva' have 'protection' strategy. The graphs show the relationship between the absolute mean of the Morris elementary effects $\mu^{*}$ and the corresponding standard deviation of the Morris elementary effects $\sigma$. Larger $\mu^{*}$ means that the corresponding parameter has a larger effect on the output. Larger $\sigma$ means either that the corresponding parameter has a non-linear effect on the output or that this parameter is involved in interactions with other parameters. For clarity of representation, only the parameters that are among either the largest ten values of $\mu^{*}$ or among the largest ten values of $\sigma$ are shown. The parameter suffixes - an and - ma correspond to 'Anastatica' and 'Malva'. 
markable differences in the sets of the most influential parameters between scenarios considering different combinations of seed dispersal strategies, e.g. scenarios with the contrasting and with the same strategies (Fig. 2.7).

The parameters of the Germination submodel appeared to be more influential. The amount of time in dormancy influenced all of the outputs of the corresponding species (Fig. 2.5; Fig. 2.6). Additionally, the parameter of 'Malva' had a large influence on the outputs of 'Anastatica' (except the number of seeds of 'Anastatica' in the scenarios with calculation of ZOI after Weiner \& Damgaard, 2006). Seed weight had a large influence on the biomass of the corresponding species (Fig. 2.8). Seed probability to germinate had a large influence on the number of adult plants and seeds of the corresponding species (Fig. 2.5; Fig. 2.6)

The influence of the parameters of the Competition and Growth submodels were considered in relation to the chosen algorithm of ZOI calculation. The relative growth rate had a large influence on the output in general. Remarkably, the influence on the output for the opposite species was greater than on the corresponding one (but the relative growth rate of 'Anastatica' had no influence on outputs
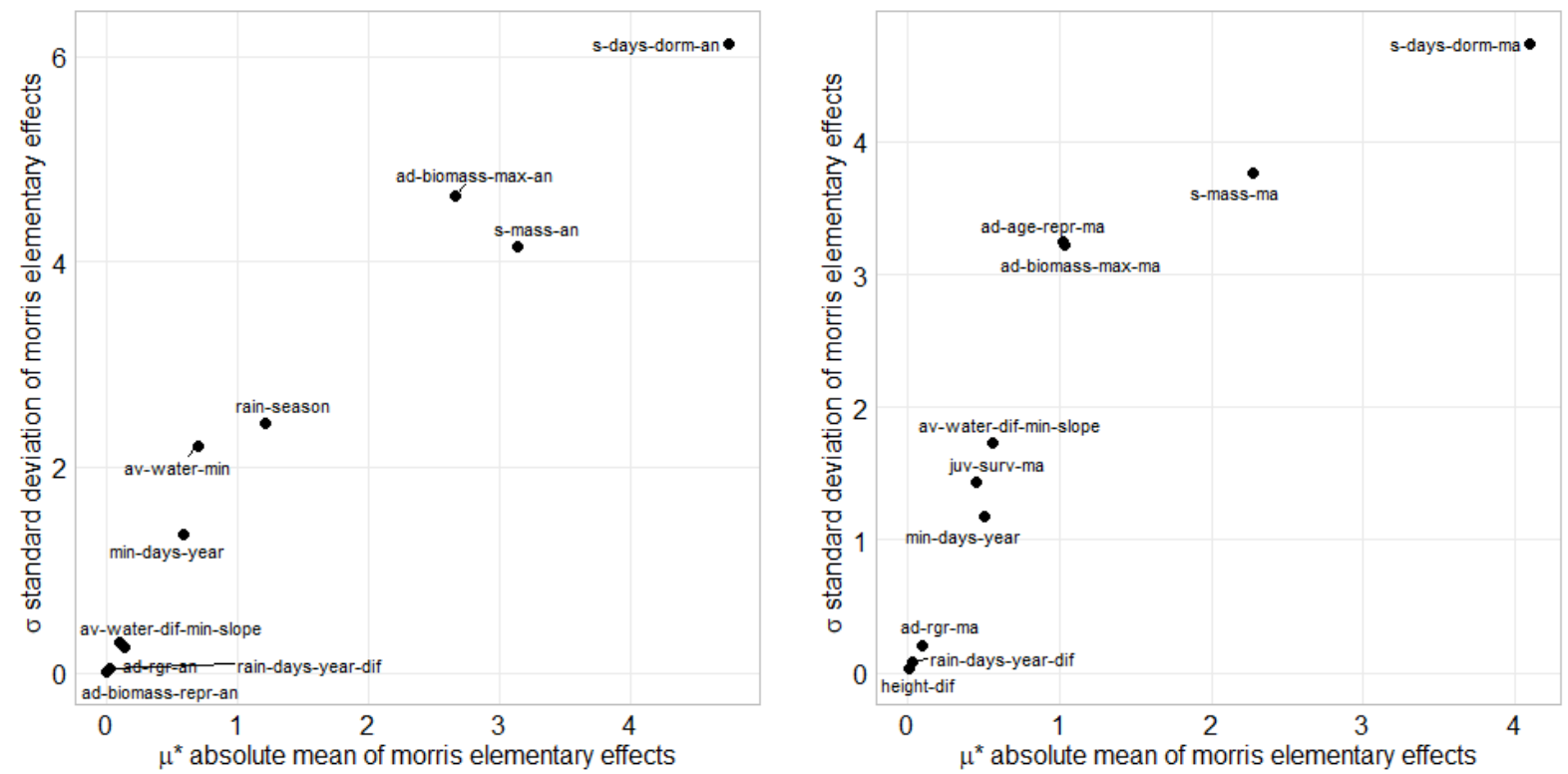

Figure 2.8. Results of the sensitivity analysis for biomass as output metric and for the scenario with ZOI-calculation after Weiner \& Damgaard, 2006 and dew absent in the model. Left panel: 'Anastatica', right panel: 'Malva', differing mainly in dispersal strategy. The graphs show the relationship between the absolute mean of the Morris elementary effects $\mu *$ and the corresponding standard deviation of the Morris elementary effects $\sigma$. Larger $\mu^{*}$ means that the corresponding parameter has a larger effect on the output. Larger $\sigma$ means either that the corresponding parameter has a non-linear effect on the output or that this parameter is involved in interactions with other parameters. For clarity of representation, only the parameters that are among either the largest ten values of $\mu^{*}$ or among the largest ten values of $\sigma$ are shown. The parameter suffixes $-a n$ and $-m a$ correspond to 'Anastatica' and 'Malva'. 
for 'Malva' in the scenarios after Weiner \& Damgaard, 2006). The maximum biomass of adult plants had a high influence on the mean biomass of the corresponding species, but maximum biomass of 'Anastatica' additionally influenced the number of adult plants, while maximum biomass of 'Malva' had a greater influence on the number of seeds. Furthermore, the maximum biomass of Malva had a high influence on the mean biomass for 'Anastatica' in the scenarios with calculation of ZOI after Lin et al., 2012 but not after Weiner \& Damgaard, 2006 (compare left panels of Fig. 2.8 and Fig. 2.9).

In general, in the scenarios with calculation of ZOI after Weiner \& Damgaard, 2006, the influence of the involved parameters on the model output of maximum biomass and relative growth rate of 'Malva' was lower than in the scenarios after Lin et al., 2012.

The comparison of the scenarios of calculation of ZOI showed that they had different sets of highly influential parameters (Fig. 2.10). In the scenarios with calculation of ZOI after Weiner \& Damgaard, 2006, water availability at local minima was a highly influential parameter for all output reference values, including the mean biomass. Furthermore, the precipitation parameters had a high influence on the output in these scenarios. It seemed that the calculation of ZOI after Lin et al., 2012
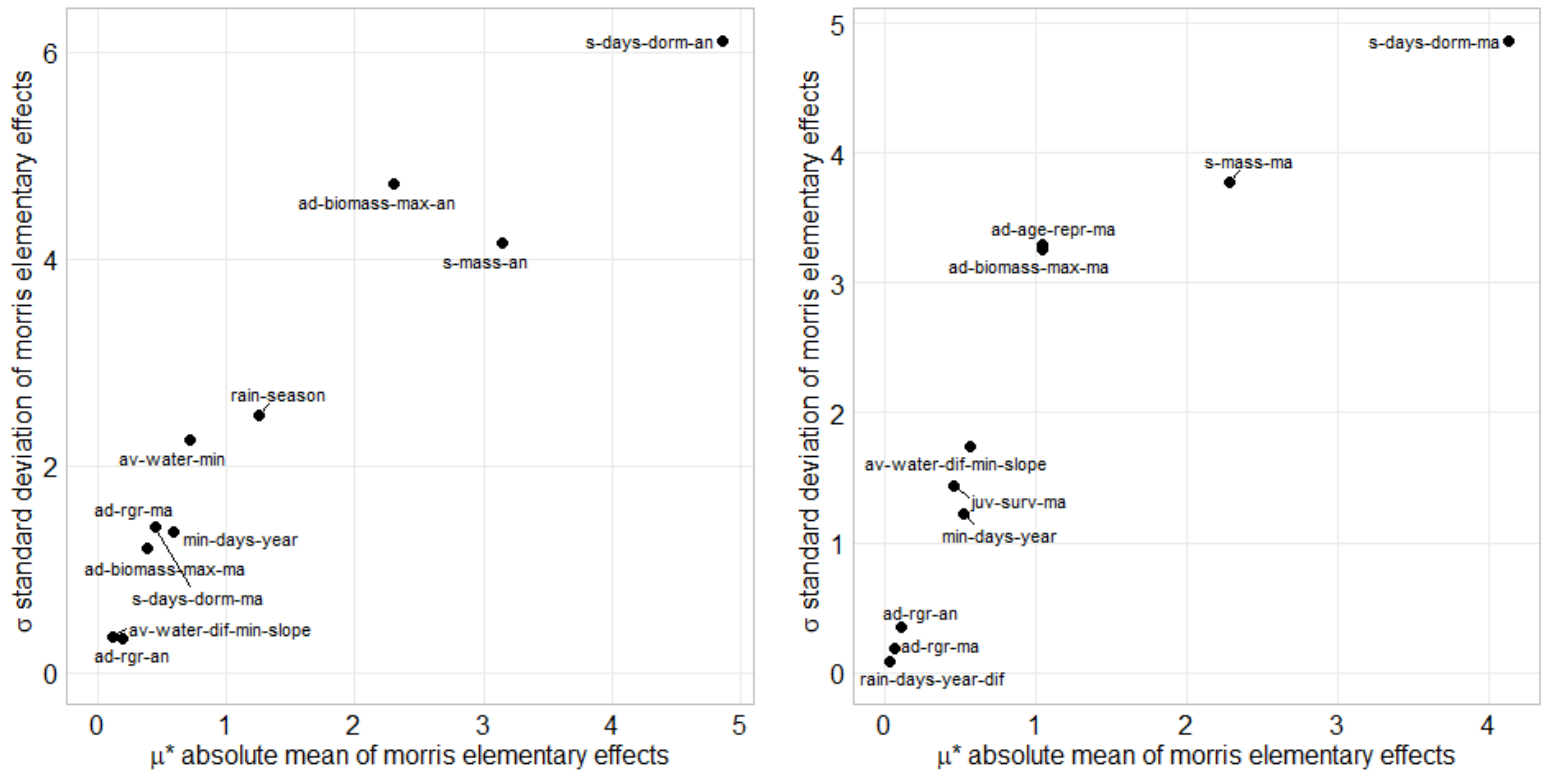

Figure 2.9. Results of the sensitivity analysis for biomass as output metric and for the scenario with ZOI-calculation after Lin et al., 2012 and dew absent in the model. Left panel 'Anastatica', right panel: 'Malva', differing mainly in dispersal strategy. The graphs show the relationship between the absolute mean of the Morris elementary effects $\mu^{*}$ and the corresponding standard deviation of the Morris elementary effects $\sigma$. Larger $\mu^{*}$ means that the corresponding parameter has a larger effect on the output. Larger $\sigma$ means either that the corresponding parameter has a non-linear effect on the output or that this parameter is involved in interactions with other parameters. For clarity of representation, only the parameters that are among either the largest ten values of $\mu^{*}$ or among the largest ten values of $\sigma$ are shown. The parameter suffixes $-a n$ and $-m a$ correspond to 'Anastatica' and 'Malva'. 
increased the overall influence of parameters from the Competition and Growth submodel on model output.

The parameters of the Reproduction submodel demonstrated different influences on the model output depending on the species dispersal strategy. The age of reproduction of 'Malva' had a high influence on all the outputs of the corresponding species (right panels of Fig. 2.5; Fig. 2.6; Fig. 2.7; Fig. 2.8). The species-specific number of seeds of 'Malva' had a high influence only on the number of seeds, while it did not have any influence on the number of adult plants and mean biomass (compare Fig. 2.6 against Fig. 2.5 and Fig. 2.7). On the contrary, the age of reproduction and the species-specific number of seeds of 'Anastatica' had either no or very little influence on model output.

In the Adult mortality submodel, the survival probability of plants had a high influence on the number of adult plants of the corresponding species. There was also some influence of the 'Anastatica' probability on the output reference value for 'Malva', while the probability to survive of 'Malva' did not influence at all any of the outputs of 'Anastatica'. The biomass threshold between seedling and adult plant stages did not markedly influence model output.
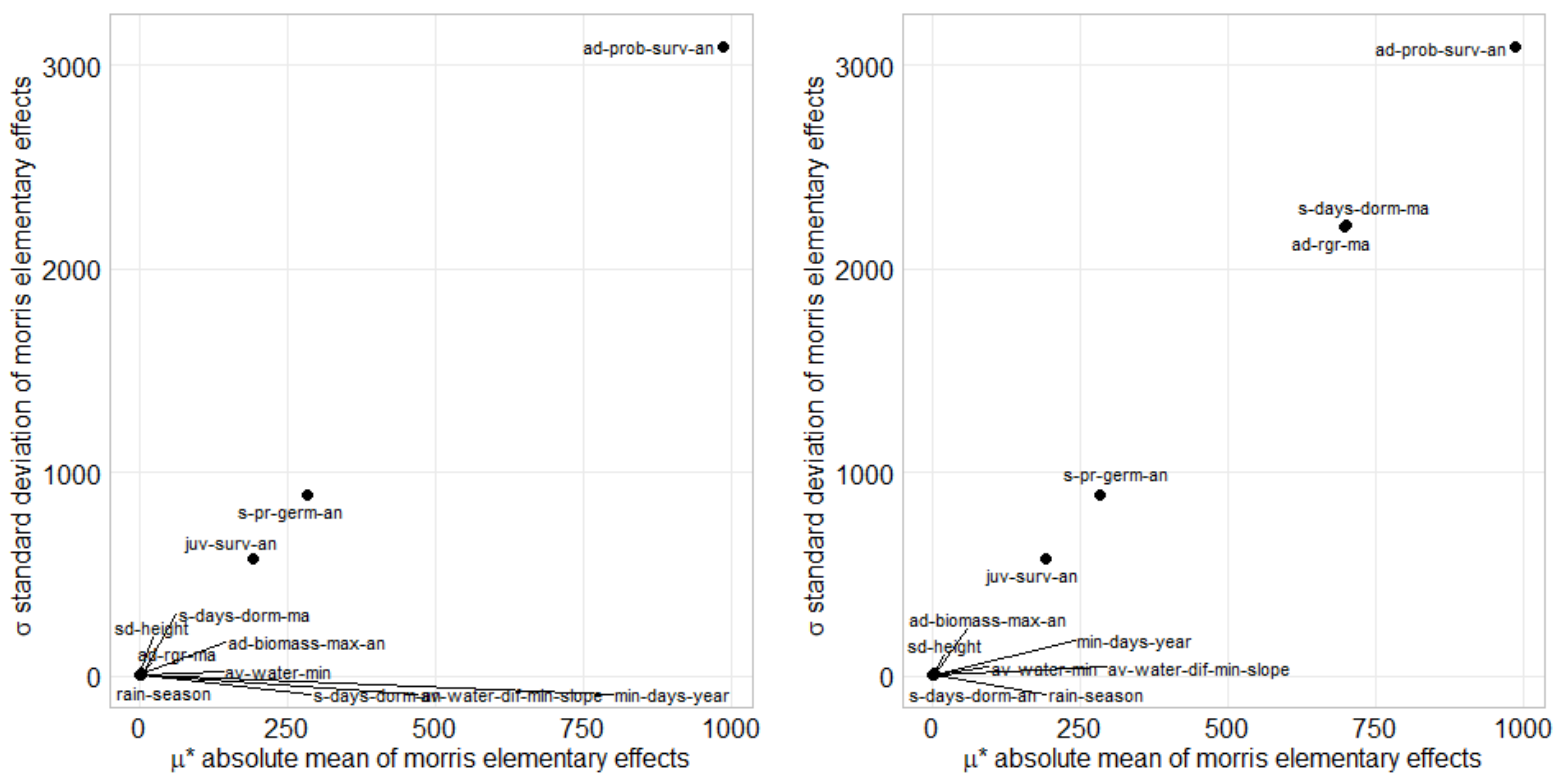

Figure 2.10. Results of the sensitivity analysis for number of adult plants for 'Anastatica' as output metric and dew absent in the model. Left panel: ZOI- calculation after Weiner \& Damgaard, 2006; right panel: ZOI-calculation after Lin et al., 2012. The graphs show the relationship between the absolute mean of the Morris elementary effects $\mu^{*}$ and the corresponding standard deviation of the Morris elementary effects $\sigma$. Larger $\mu^{*}$ means that the corresponding parameter has a larger effect on the output. Larger $\sigma$ means either that the corresponding parameter has a non-linear effect on the output or that this parameter is involved in interactions with other parameters. For clarity of representation, only the parameters that are among either the largest ten values of $\mu^{*}$ or among the largest ten values of $\sigma$ are shown. The parameter suffixes $-a n$ and $-m a$ correspond to 'Anastatica' and 'Malva'. 


\subsection{Discussion}

In this paper, we present an individual- and trait-based model simulating desert plant community dynamics. With this model, it is possible to study the influence of plant functional traits and environmental factors on the community dynamics of annual plants. The key aim of the model is to understand processes rather than to make forecasts (model for demonstration sensu Evans et al., 2013). Despite of the fact that there are some known models describing desert vegetation (James et al., 2005) or desert vegetation being a special case of a large vegetation model (Reick et al., 2013), to our knowledge, the model presented in this paper is the first one with a special focus on community dynamics of desert annuals. The key drivers of community dynamics in our model scenarios were more often plant functional traits than environmental factors. These traits were related to competition, growth, germination and survival, but surprisingly much less to dispersal. Environmental factors driving community dynamics in the model were based on soil water availability and precipitation. This is in accordance with the proposed environmental drivers of actual desert communities (Hegazy and Kabiel, 2007). The proof-of-principle simulation reproduced accurate pulse dynamics of desert annual plant communities. This shows that the model is structurally realistic. The model can be parameterized to mimic, complement and extend controlled greenhouse experiments. This approach would contribute to not only model development but also facilitate empirical experiments, which are often restricted in the possible complexity of the experimental design and sample size (Meyer et al., 2009; Radny et al., 2018; Radny and Meyer, 2018). To increase the reliability of the model, we suggest further validation of the model by comparing model results first against data from one field site, then against several field sites, then against data from a different desert ecosystem.

The results of the sensitivity analysis highlighted the relative importance of different environmental parameters and plant functional traits for a range of model outputs. Among the environmental factors, the most important factors were precipitation, the minimum number of rainy days and the length of the rainy season, as well as soil water availability at sites with expected higher water availability (local minima and slopes). When dew effects were included in the model, the parameters defining dew influence, such as the corresponding increase in water availability and the duration of the period of dew deposition, had a high influence on the model output. Based on these implied dew effects 
on the model output, we advocate for a more elaborated dew submodel in future model versions with more detailed effects on plant growth. The topographical parameters affected model outputs only indirectly, via water availability. Taken together, this is in line with water as the main limiting environmental factor in deserts.

The influence of plant functional traits on model output differed from submodel to submodel and between the generic species. The parameters from competition and growth submodels such as the relative growth rate and maximum biomass were among the most influential parameters across all the considered scenarios. These two parameters are plant functional traits that are involved in plant-plant interactions modelled via the calculation of ZOIs. The comparison of the scenarios applying different calculations of ZOIs demonstrated that this choice not only directly influenced how biomass is built into the model architecture, but also changed the sensitivity of the entire model to the other parameters (Fig. 2.10). In accordance with these results, we advocate that the choice of the ZOI approach in the model should be based on empirical measurements of the relationship between biomass and growth for each considered species or group of species. If these measurements are not available, model calibration techniques or pattern-oriented modelling (Grimm, 2005) can be applied to select the suitable calculation of ZOI.

The germination and adult mortality submodels incorporated several highly influential parameters: amount of time in dormancy, probability to germinate, and plant probability to survive. These plant functional traits are related to the ability of plants to survive in desert environments. Although the importance of such germination- and survival-related traits was expected, it was still surprising that these traits were much more important than any dispersal-related traits or strategies in the model. This means that coping with the local environmental conditions is much more important than exploring new environments, at least in our model communities. This disagrees with informed-dispersal theory, which claims that organisms prefer to avoid stressful local environment by investing their costs in dispersal (Martorell and Martínez-López, 2014). We hypothesize that the greater importance of tolerance versus avoidance strategies (in the form of dispersal) will decrease with increasing spatial heterogeneity of the environment and that this relationship will depend on the scale of the heterogeneity. We sug- 
gest testing this hypothesis in future simulation experiments with model versions that differ in spatial heterogeneity across spatial scales.

Based on the insights from the sensitivity analysis, refinement of the model parameters would be the next logical step of further model development. Highest priority in parameter refinement should usually be given to the high-sensitivity parameters, i.e. amount of time in dormancy, probability to germinate, probability to survive, relative growth rate, maximum biomass, soil water availability, and precipitation parameters. Parameter refinement relies on new or refined empirical measurements. However, not all of the high-priority parameters will be easy to measure; some may even turn out to be impossible to measure in practice. Traits that are hard to measure can be approximated by 'soft traits' that are easier to measure (Hodgson et al., 1999; Cornelissen et al., 2003) or easier to model. For example, root traits are very hard to measure and were approximated by zones of influence in our model. Similarly, seed dispersal parameters can be derived from the spatial distribution of plants relative to the mother plant (Nathan and Muller-Landau, 2000).

Where it is impossible to obtain parameter values, these gaps might be filled with the help of pattern-oriented modelling (Wiegand et al., 2003; Grimm, 2005). If applied for inverse parameterization, this approach may assist in acquiring plant functional traits that are not available in the literature, are hard to measure in the field and cannot be easily replaced by 'soft traits'. For example, the output reference values in the sensitivity analysis such as biomass distributions and numbers of adult plants would be the first candidate patterns to match with observed natural patterns. Another pattern for comparison with real-world observations would be the spatial distribution of individuals with different species identities. Of course, if the aim of the model was changed from understanding mechanisms towards forecasting concrete community dynamics, different priorities in parameter refinement may arise. For forecasts, the model should be run with real-world rainfall data from meteorological stations and corresponding height-above-sea-level values from digital elevation models of a reference site instead of the currently used algorithms generating environmental input. For understanding general mechanisms, the current model version seems to be valid and applicable, but parameter refinement will certainly improve the reliability of its results. 
Our model combines trait-based and individual-based approaches. This combination provides an opportunity to derive community properties from the functional traits of individual plants, interactions between these individuals and between the individuals and their environment (Zakharova et al., 2019). Moreover, as a mechanistic model, the presented model will contribute to our understanding of the processes that affect desert plant communities. As shown in the model, these processes are both associated with the interaction between plants and their environment and inter- and intraspecific plantplant interactions. We combined a trait-based approach with an individual-based approach by assigning plant functional traits to individual agents in the model, with traits being directly linked to a strategy or an organismal function (Violle et al., 2007). Simultaneously, the bottom-up principle of individual-based models (Grimm and Railsback, 2005) led to the emergence of population- and communitylevel dynamics from interacting traits and individuals (Salguero-Gómez et al., 2018). Modelling these individual biotic interactions (e.g. competition) or abiotic interactions (e.g. individual responses to the fast-changing desert environment) was possible because of the individual- and grid-based nature of the model (Jeltsch et al., 2008). Using a combined trait-based and individual-based approach also improved the mechanistic tractability of the model.

The model presented in this paper can be considered as a general framework for future case studies. We advocate for future elaboration and extension of the model by incorporating more detail at the submodel level and by introducing new submodels. For example, explicitly including soil properties and plant water uptake will refine the water-availability submodel. A future extension to the model might consider salinity effects on soil properties, water availability and water uptake, depending on precipitation, evaporation and vegetation. Submodels including the most influential parameters according to the sensitivity analysis such as competition, growth and germination submodels are of particular interest. In line with claims for more studies of intraspecific trait variation (Violle et al., 2012; Burton et al., 2017), it is principally possible to vary trait values between individuals in our model due to its individual-based approach. With these additional details, the model can be used to tackle several research questions, including studying communities under different climates representing IPCC scenarios (IPCC, 2018). 
In summary, the model presented here is the first attempt to study plant community dynamics of desert annual species with the help of a combined trait- and individual-based approach. The model suggests paths to deal with two major challenges: lack of empirical data and difficulty to capture the ever-changing environmental conditions that desert plant communities face. The lack of data can be addressed by using trait-based approaches and incorporating pattern-oriented modelling to acquire parameter values that are hard to measure. The spatial and temporal heterogeneity of desert communities is tackled with the individual-based modelling approach. In this way, the model presented here will advance the research of desert plant communities and understanding their fate under global changes.

\section{Acknowledgements}

This research was funded by "Niedersächsisches Vorab" (MWK ZN3155) and Volkswagen Foundation. We are grateful to Fabian Slowik, Maria Fernanda Arroyave Martinez, and Nir Krintza from Ben-Gurion University of the Negev, Israel for providing plant functional trait data and further insights based on experiments and fieldwork. We used the 'sequence-determines-credit' approach for author order. 
Chapter 2.

Combining trait- and individual-based modelling to understand desert plant community dynamics 
Chapter 3. The success of plant survival strategies under spatial and temporal heterogeneity in warm deserts: a simulation experiment

This chapter is a manuscript to be submitted as a research article. The authors: L. Zakharova, K.M. Meyer, M. Seifan

Authors' contribution: L.Z., K.M.M. and M.S. conceived of the presented idea. L.Z. and K.M.M. designed, conducted and analysed the simulation experiment. L.Z. led the writing of the manuscript with extensive input from K.M.M. and M.S..

\begin{abstract}
Heterogeneity of the environment limits the life of desert annual plants. Desert annual plants acquired different strategies to survive under high heterogeneity, predominantly targeted at the seed phase. The key seed strategies are an escape in time strategy and an escape in space strategy, which have been linked to survival under respectively temporal and spatial heterogeneity. We suggest using plant functional traits involved in these seed escape strategies to connect them to the colonizationcompetition trade-off. This way they align with what was previously coined 'protection' and 'escape' seed strategies of desert plants. Using a spatially explicit simulation model, we investigated the survival and performance of two annual plant species as representatives of a protective-competition strategy and an escape-colonization strategy. The results confirmed the dominance of the escapecolonization strategy in the earlier phase of community dynamics, while the protective-competition strategy dominated in the later phase. Additionally, the simulation results showed the superiority of the protective-competition strategy under high temporal heterogeneity, which relaxed under high spatial heterogeneity. The insights from this simulation experiment advocate for a trait-based description and integrated consideration of life phases to capture plant survival strategies and their success in heterogeneous desert landscapes.
\end{abstract}




\subsection{Introduction}

Deserts are known as environments with organisms adapted to extreme conditions (Ward, 2009). These environments challenge their inhabitants by high heterogeneity in time and space (Chesson, 2000; Venable et al., 2008). For example, the Negev desert has high inter- and intraannual variation in rainfall patterns (Berkowicz et al., 1995), which affects the survival of plants (Tielbörger and Kadmon, 2000). In addition to the temporal variability, spatial heterogeneity also affects plant performance in deserts (Loik et al., 2004; Hegazy and Kabiel, 2007). Annual plants seem to be particularly vulnerable to these rapid environmental changes as they have only one growth season for the entire life cycle (but see Salguero-Gómez et al., 2012). This group of plants developed intrinsic adaptations to the everchanging conditions of deserts, demonstrating a shorter growth cycle and plasticity in the transitions from one life cycle stage to another (Aronson et al., 1993).

Strategies targeted at the seed stage seem to be reliable promises for annual plant survival in deserts under temporal and spatial heterogeneity, as they ensure the survival over a specific unfavourable growth season and therefore the persistence of the entire population in a certain region (Venable and Lawlor, 1980). Plant strategies for increasing the chances of seeds to survive and germinate in variable and heterogeneous environments such as deserts involve variability in dispersal strategies and timing of germination (Venable and Brown, 1988). Particularly, desert annuals have for many years been model organisms to study bet hedging, a phenomenon of sacrificing current performance to ensure success in the future, and a number of studies were conducted to understand the underlying mechanisms of these survival strategies (Tielbörger and Valleriani, 2005; Gremer and Venable, 2014). Plants invest usually in one of two bet-hedging strategies: seed dormancy or seed dispersal (Venable and Brown, 1988; Snyder, 2006). Thus, these two main strategies aim to avoid periods of unsuitable conditions by escaping in space or in time in form of a seed (Venable and Lawlor, 1980). Escape in space increases the chances of finding more suitable habitats, while escape in time contributes to survival until conditions become more favourable in situ. Although both strategies can potentially contribute to the survival of annual plants in deserts, it was suggested that escape in time is more important for desert plants (Adondakis and Venable, 2004; Venable et al., 2008). The rationale behind 
this statement is that precipitation seems to vary more than any spatial factor in deserts (Venable and Lawlor, 1980; Ellner and Shmida, 1981). However, others have suggested that escape in space might be as important for desert plants, because it is much easier to realize in deserts: Escape in space relies on runoff and wind, which are promoted by the openness of desert landscapes (Mott and McComb, 1974; Reichman, 1984). Thus, it is still not clear, under which environmental conditions escape in space or escape in time are more relevant for plant survival.

Pure escape-in-time or escape-in-space strategies are rare in nature. Many species exhibit mixed strategies that include seed dormancy and seed dispersal at varying degrees (Adondakis and Venable, 2004; Oudtshoorn and Rooyen, 2013), which are sometimes combined with strategies targeting biotic interactions such as competition. For example, larger seed size has been associated with the increased ability of plants to compete, while smaller size and greater number of seeds per individual facilitates colonization (Leishman, 2001; Turnbull et al., 2004). This difference in seed size has been linked to a colonization-competition trade-off (Levins and Culver, 1971; Tilman, 1994), where largeseeded species are seen as superior competitors but inferior colonizers and vice versa (Rees, 1995; Turnbull et al., 1999). If seed escape strategies are viewed in the framework of the colonizationcompetition trade-off, the escape-in-time strategy accounts for superior competitors, whereas the escape-in-space strategy is adopted by superior colonizers that can avoid competition with other plants if we assume that the environment offers competitor-free space at least at some locations. For example, spatial processes that affect seed dispersal were suggested to promote the persistence of an inferior competitor (Weiner and Conte, 1981; Bolker and Pacala, 1999). Escape-in-time strategists are likely prone to much higher local competitive pressure than escape-in-space strategists. Furthermore, competition plays an important role in environments with high spatial and temporal unpredictability, especially when the rainfall arrives (Chesson et al., 2004). The right timing of germination out of seed dormancy contributes to future competitive success among annuals (Chesson et al., 2004). Thus, it seems to be promising to link seed escape strategies in deserts and the colonization-competition tradeoff. To our knowledge, the relationship between seed dispersal strategies and the colonizationcompetition trade-off has not yet been explored for desert annual species. 
Two strategies suggested for annual plants in the Negev desert by Gutterman (1994) to some extent capture characteristics of both escape-in-time/ escape-in-space strategies and the competitioncolonization trade-off. The first strategy, the 'protection strategy' (Gutterman, 1994), is adopted by plant species with fewer larger seeds, which conveys superior competitiveness in the competitioncolonization trade-off (Turnbull et al., 2004). The protection strategy can also be considered as a special form of the escape-in-time strategy. In contrast to other escape-in-time strategies, the protection strategy ensures escape in time before seed dispersal and not afterwards. The large seeds of plants with the protection strategy are dispersed in small portions only when there is water available for plant growth in the environment (Ellner and Shmida, 1981). The second strategy, the 'escape' strategy (Gutterman, 1994), follows mainly an escape-in-space strategy by producing many small seeds that facilitate seed dispersal and, thus, represents superior colonizers in the competition-colonization trade-off (Turnbull et al., 2004). For the purpose of this paper, where we suggest to link seed escape strategies with the colonization-competition trade-off, we recoin Gutterman's (1994) protective strategy as a 'protective-competition' strategy and his escape strategy as an 'escape-colonization’ strategy.

In this research, we conducted a simulation experiment with the trait- and individual-based spatial-explicit model of annual plant communities ATID (chapter 2). This model simulates different seed dispersal mechanisms as well as competition between annual plants. We focus on a two-species community as it is observed in several sites across the Negev desert. This community consists dominantly of two annual species, Anastatica hierochuntica L. (Brassicaceae) and Malva parviflora L. (Malvaceae). Anastatica hierocuntica is a classical representative of the protection strategy (Gutterman, 1994) and escape-in-time strategy (Venable and Lawlor, 1980), where seeds are protected on the mother plant and dispersed in portions only under raindrops (Friedman and Stein, 1980). Thus, the protective-competition strategy can be attributed to A. hierochuntica. Dispersed seeds usually germinate without any further delay. The escape strategy of Malva parviflora can be seen as a combination of escape in time and space. The species produces numerous small seeds that can be eaten by sheep and, thus, dispersed to longer distances. Additionally, seeds of M. parviflora have a pronounced dor- 
mancy period (Michael et al., 2006) ensuring that not all seeds germinate at the same time. Accordingly, M. parviflora is considered in this study as a representative of the escape-colonization strategy.

Our aim was to explore the survival and performance of these two species as representatives of different survival strategies of annual plants in the Negev desert and put that into relation with the escape-in-space/escape-in-time strategies and the colonization-competition trade-off. By choosing to implement real species instead of theoretical species with 'pure' strategies, we ensure the biological realism and the applicability of our results. We exposed these species to four scenarios representing pairwise combinations of high or low spatial heterogeneity and high or low temporal heterogeneity. The range of these heterogeneities lies within the ranges observed across the Negev desert and in warm deserts in general. We expect that the protective-competition strategy (1) is superior to the escape-colonization strategy under high temporal heterogeneity (and thus unpredictability); (2) is inferior to the escape-colonization strategy under high spatial heterogeneity (and thus unpredictability); and (3) is linked to successful competition measured as long-term persistence in a local community and that the escape-colonization strategy is linked to successful colonization measured as short-term dominance in a local community.

\subsection{Methods}

\subsubsection{Study area}

Model input is based on data originating from the Negev desert. For model parameterization and calibration, we focused on the site Meishar (30²4'48.3"N 3456'37.9"E) near Mizpe Ramon, Israel, applying a digital elevation model from this site and precipitation information. The initial conditions with respect to precipitation ("The Meteorologic Service Database, Israel Government Portal," 2020), topographic data (unpublished), and plant community composition reflect field observations from Meishar.

The functional trait values for the modelled species were determined for two major annual species in the plant community in Meishar: Anastatica hierochuntica and Malva parviflora as captured 
during field observations in the growth season in 2017. Plant functional trait values assigned to these species were taken from the literature and expert knowledge based on field observations and nethouse experiments (Appendix. Tab. A1). Species composition and abundance of each species used for model calibration were measured within 1 by $1 \mathrm{~m}$ quadrats, with a distance of $5 \mathrm{~m}$ from each other at a study site at Meishar with side lengths of $100 \mathrm{~m}$ by $50 \mathrm{~m}$.

Model scenarios were based on simulated input within margins of environmental conditions that are typical of those parts of the Negev desert with a mean annual precipitation of less than $80 \mathrm{~mm}$ (Berkowicz et al., 1995).

\subsubsection{Model parameterization and calibration}

We used environmental data derived from topographic data (unpublished) of Meishar and meteorological data from the study site as an input to the ATID-model (chapter 2), developed in NetLogo (Wilensky, 1999), version 6.0.2.

The model was parameterized for two species expressing different survival strategies: Malva parviflora and Anastatica hierochuntica. The species differ substantially in their seed dispersal mechanisms, seed mass, number of seeds produced per individual and in relative growth rate (Appendix. Tab. A3.1). This two-species case of a plant community was observed during the fieldwork in May 2017, when the number of individuals of other species was negligible (all other species $<1 \%$ abundance). We extrapolated the complete counts of plant individuals in 401 by 1 m quadrats to the area of the full study site with side lengths of $100 \mathrm{~m}$ by $50 \mathrm{~m}$, yielding 371,125 adult plants of $A$. hierochunti$c a$ and 246,500 adult plants of M. parviflora plants. We used these values to calibrate the model with a special focus on the most uncertain parameters with respect to literature and expert knowledge: number of seeds at the beginning of the simulation, relative growth rate and maximum biomass of both species. These parameters were also associated with high sensitivities in the sensitivity analysis of the model (chapter 2). We ran a calibration procedure for individual-based simulation models with $\mathrm{R}(\mathrm{R}$ Core Team, 2018; version 3.5.1) using the package "nlrx" (Salecker and Sciaini, 2019; Salecker et al., 
2019) to find the corresponding parameter values (Appendix. Tab. A3.1). The calibration was based on an Approximate Bayesian computation algorithm coupled with an MCMC sequential scheme after Marjoram et al. (2003) as it is implemented in the R package EasyABC (Jabot et al., 2013; Jabot et al. 2015).

\subsubsection{Scenarios}

We defined the environmental conditions at the study site in Meishar as neutral scenario. For the four main scenarios in this analysis, we simulated respectively either higher or lower temporal and spatial heterogeneity than in this site based on the topography and rainfall simulators provided by the ATIDmodel (Tab. 3.1). The ranges of the environmental parameters implemented in the scenarios were artificial to create clearly different conditions. However, the ranges of the parameters were kept realistic with respect to rain data obtained from meteorological observations in this part of the Negev desert ("The Meteorologic Service Database, Israel Government Portal," 2020) and height difference information (topographic data, unpublished). Soil water availability was kept the same for all scenarios as soils in this part of the Negev have similar properties (Dan et al., 1976). The length of the rain season was also constant over all scenarios. For all four scenarios, we simulated two-species communities representing species with different survival strategies. The simulations were run until the death of all the plants and seeds on a simulated site with side lengths of $100 \mathrm{~m}$ by $50 \mathrm{~m}$. Number of seeds, number of plants and mean biomass of adult plants were recorded at each time step (one day) to measure survival and performance of both species. 
Table 3.1. The parameter values of the four simulated scenarios with respect to height difference and standard deviation (sd) of the surface and interannual difference in the number of rain days. For comparison, these are the parameter values for the neutral scenario corresponding to the 'Meishar' site: height difference $=5 \mathrm{~m}$, height $\mathrm{sd}=0.05 \mathrm{~m}$; interannual rain days' difference $=30$ days (see Appendix. Fig. A3.1 for simulation results for the 'Meishar' site).

\begin{tabular}{lll}
\hline & Low spatial heterogeneity & High spatial heterogeneity \\
\hline Low temporal heterogeneity & Scenario A. Height difference $=2 \mathrm{~m}$, & Scenario B. Height difference $=8 \mathrm{~m}$, \\
& height sd $=0.01 \mathrm{~m}$; interannual rain & height sd $=0.1 \mathrm{~m}$; interannual rain days \\
& days difference $=20$ days & difference $=20$ days \\
Scenario C. Height difference $=2 \mathrm{~m}$, & Scenario D. Height difference $=8 \mathrm{~m}$, \\
height $\mathrm{sd}=0.01 \mathrm{~m}$; interannual rain & height sd $=0.1 \mathrm{~m}$; interannual rain days \\
days difference $=40$ days & difference $=40$ days \\
\hline
\end{tabular}

\subsection{Results}

Simulated two-species communities existed for 18-28 years. The longest simulations were recorded for scenario D, with high spatial and temporal heterogeneity (Fig. 3.1 - 3.3). This scenario also ensured the longest coexistence of two species, followed by scenario A, with low spatial and temporal heterogeneity (Fig. 3.1-C and 3.1-A). The number of plants (Fig. 3.1) and the number of seeds (Fig. 3.2) for simulated Malva parviflora (escape-colonization strategy) consistently decreased during the simulation, whereas the respective numbers for Anastatica hierochuntica (protective-competition strategy) remained relatively stable over the years. Mean biomass of adult plants reached its maximum after 7-8 years of the simulation runs across all the scenarios (Fig. 3.3). This maximum in mean biomass matched in time with the relative equalization in the numbers of seeds and plants between the two simulated species. Thus, over all scenarios, two clear phases of coexistence of the two species were observed. 

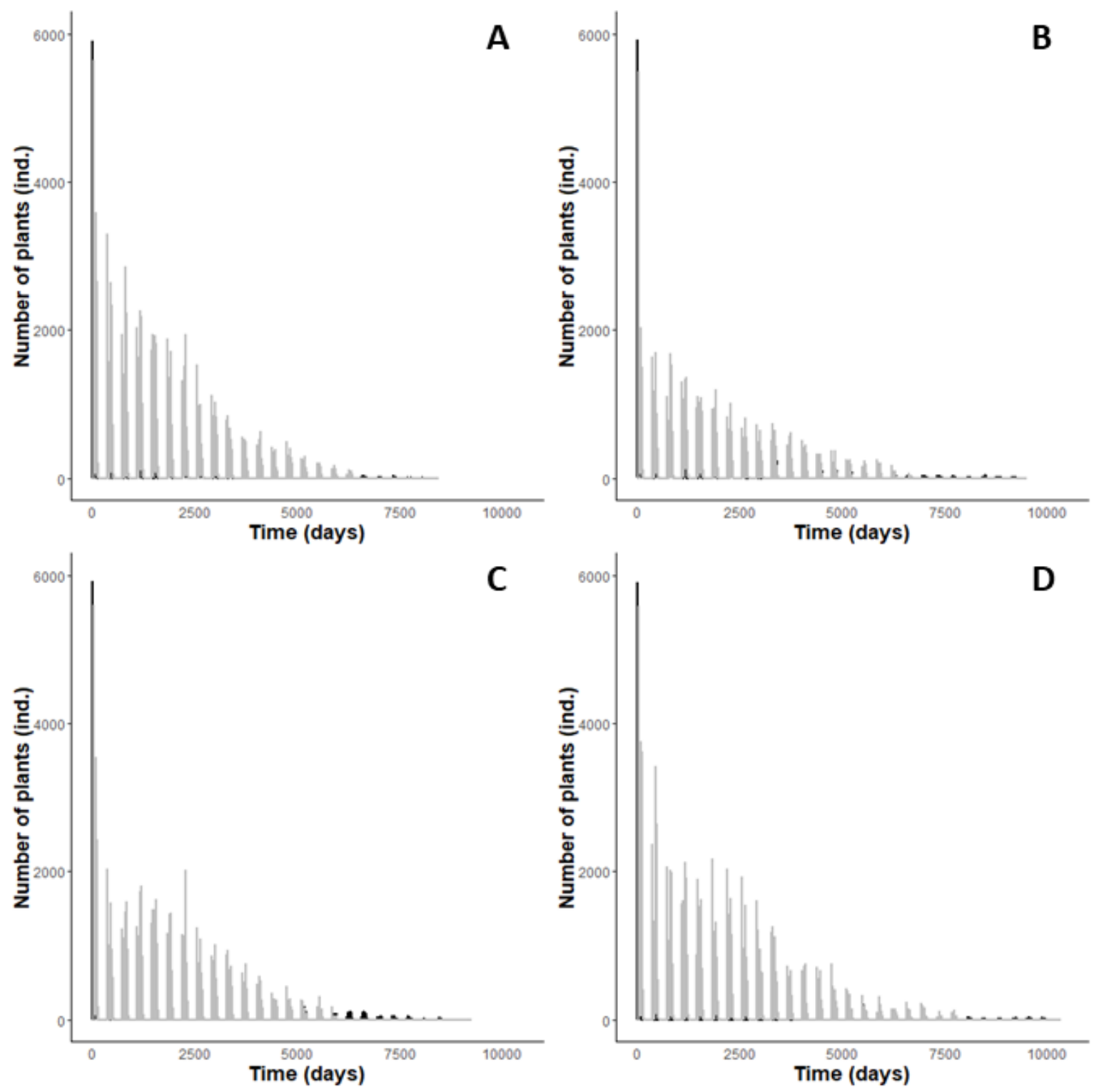

Figure 3.1. Number of plant individuals of the species with the protective-competition strategy (black) and with the escapecolonization strategy (grey) over simulated time in four scenarios (A: low temporal and spatial heterogeneity, B: low temporal heterogeneity and high spatial heterogeneity, C: high temporal heterogeneity and low spatial heterogeneity, D: high temporal and spatial heterogeneity).

In the first phase of the simulation, the escape-colonization strategy was superior to the protective-competition strategy in the number of plants (Fig. 3.1), the number of seeds (Fig. 3.2) and mean biomass of adult plants (Fig. 3.4). In the second phase, the protective-competition strategy was dominant and on average was associated to the last species to survive in the given environment (but see separate simulation runs: Appendix. Fig. A3.2). 

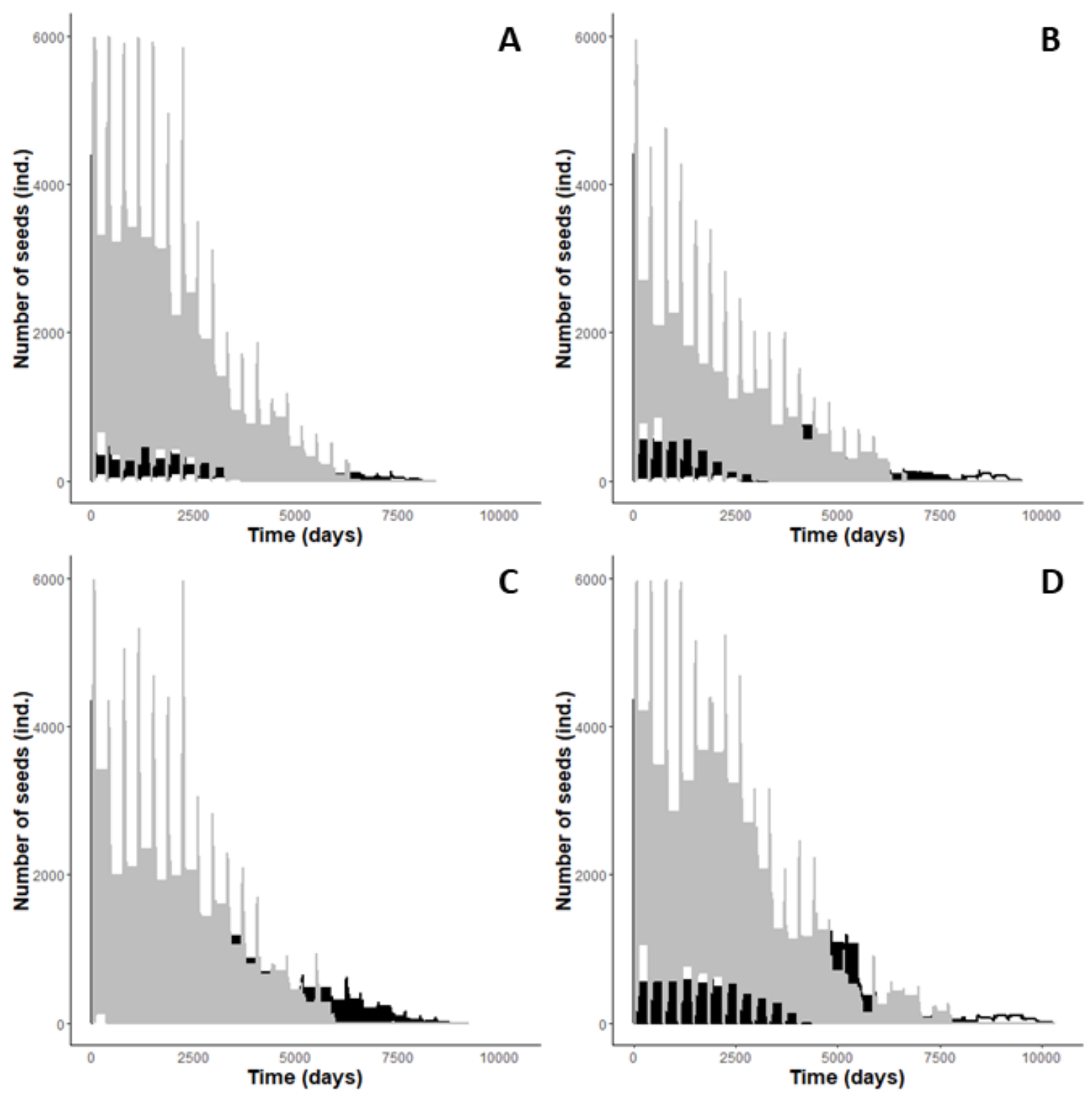

Figure 3.2. Number of seeds produced by the species with the protective-competition strategy (black) and with the escapecolonization strategy (grey) over simulated time in four scenarios (A: low temporal and spatial heterogeneity, B: low temporal heterogeneity and high spatial heterogeneity, C: high temporal heterogeneity and low spatial heterogeneity, D: high temporal and spatial heterogeneity).

Under low spatial heterogeneity (Fig.3-A and -C), mean biomass of the species with the protective-competition strategy in the second phase of the simulation remained high up to the end. Under high spatial heterogeneity (Fig. 3.3-B and -D), however, mean biomass of the species with the protective-competition strategy started to decrease after the species with the escape-colonization strategy was outcompeted from the community.

In scenario $\mathrm{C}$ combining low spatial heterogeneity and high temporal heterogeneity, the switch to the dominance of the protective-competition strategy clearly coincided with the transition 
from the first to the second phase of the simulation (Fig. 3.4). For other scenarios, this period was more extended, accompanied by spikes in mean biomass of the species with the escape-colonization strategy (Fig. 3.3 and 3.4). Under high spatial heterogeneity (Fig. 3.3 and 3.4; scenarios B and D) in the second phase of the simulation, the species with the escape-colonization strategy had clear years of dominance over those with the protective-competition strategy up to the point of its extinction.
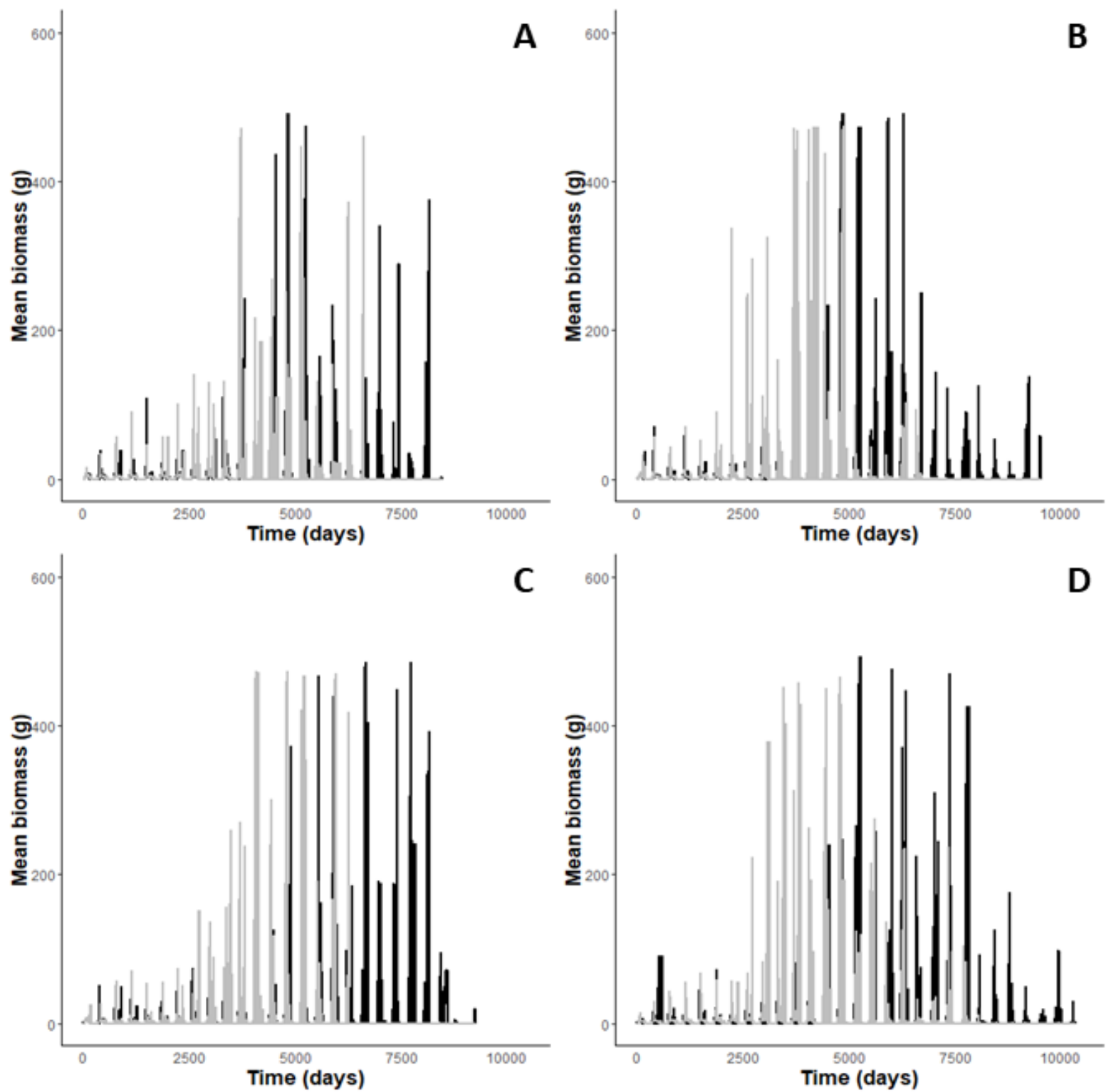

Figure 3.3. Mean biomass $(\mathrm{g})$ of the species with the protective-competition strategy (black) and with the escapecolonization strategy (grey) over simulated time in four scenarios (A: low temporal and spatial heterogeneity, B: low temporal heterogeneity and high spatial heterogeneity, C: high temporal heterogeneity and low spatial heterogeneity, D: high temporal and spatial heterogeneity). 


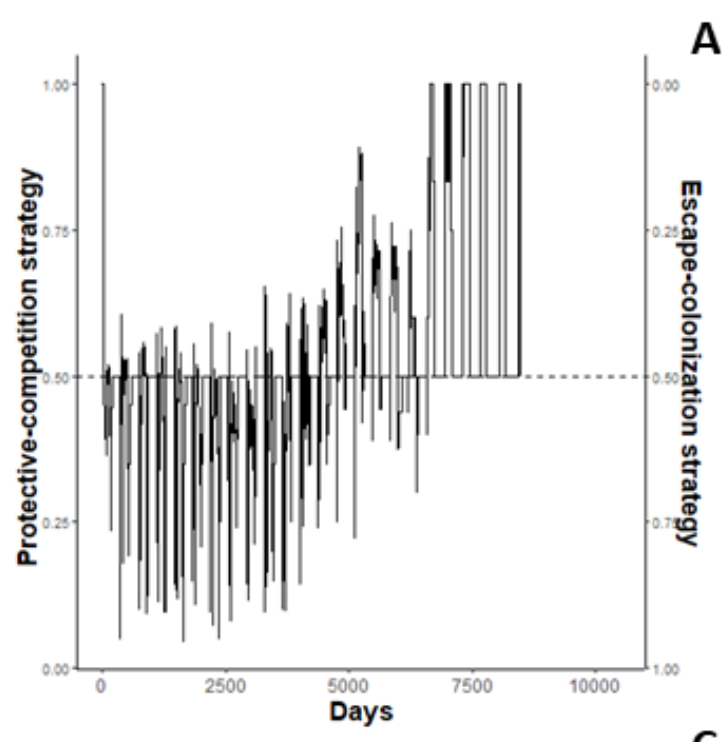

A
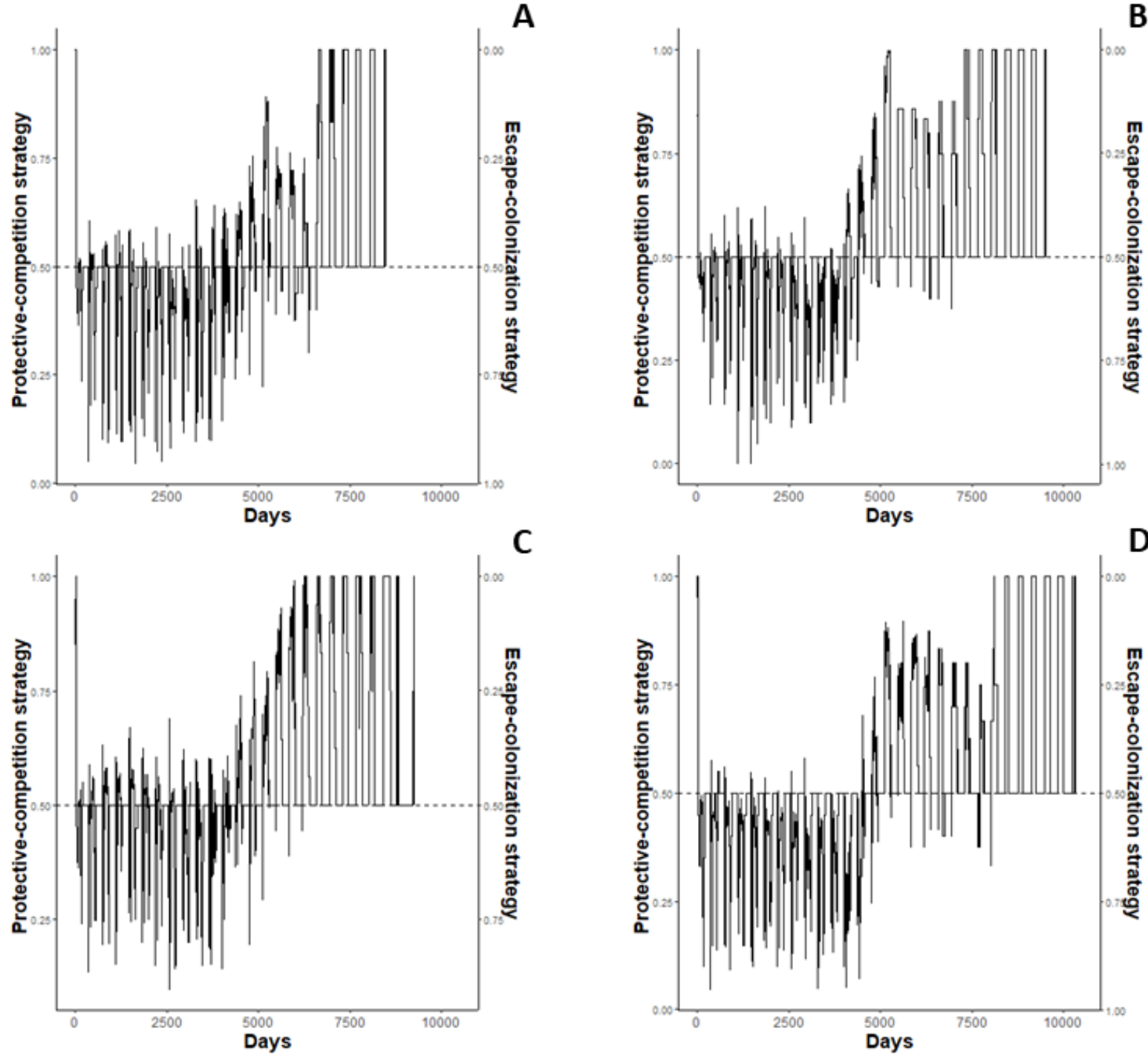

Figure 3.4. Proportion of the mean biomass of the species with the protective-competition strategy (left axis) and with the escape-colonization strategy (right axis) over simulated time in four scenarios (A: low temporal and spatial heterogeneity, B: low temporal heterogeneity and high spatial heterogeneity, C: high temporal heterogeneity and low spatial heterogeneity, D: high temporal and spatial heterogeneity).

\subsection{Discussion}

In this study, we linked seed escape strategies and the colonization-competition trade-off to explore the survival and performance of plant species representing the protective-competition strategy and the escape-colonization strategy in a two-species community of desert annuals with the ATID-model (chapter 2). We assessed the performance of the populations in terms of abundance and biomass. Biomass patterns reflected the ongoing processes, while changes in abundance were rather a delayed consequence of these processes for the community. Exposing the two strategies to high and low spatial 
and temporal heterogeneity, we found small differences between the two strategies across the scenarios and a general two-phase pattern in the community dynamics over all scenarios with the escape-colonization strategy dominating on the short term and the protective-competition strategy showing longer persistence.

Only in the second phase of the community dynamics, the relative dominance of the species with the protective-competition and the escape-colonization strategies were in line with our first two expectations. Although the protective-competition strategy was ultimately superior to the escapecolonization strategy in all scenarios, the superiority was clear from the beginning of the second phase only in the scenario with high temporal heterogeneity and low spatial heterogeneity (see scenario $\mathrm{C}$ in Fig. 3.4). This agreed with the expectation that high temporal heterogeneity leads to superiority of the protective-competition strategy. In both high-spatial-heterogeneity scenarios, the escape-colonization strategy showed dominance (even if only transiently) as predicted by our second expectation. Thus, in the scenario with high spatial and temporal heterogeneity (scenario D), where both strategies might have been expected to be superior, the escape-colonization strategy won, at least on the short term. This is probably due to the fact that the escape-colonization strategy included an, albeit weak, escape in time by exhibiting some seed dormancy. This may indicate that mixed strategies are beneficial for survival in heterogeneous environments, and especially where both spatial and temporal heterogeneity are high (Adondakis and Venable, 2004; Oudtshoorn and Rooyen, 2013; Volis and Bohrer, 2013). These results are also in line with the claim that both increases in seed size and dormancy are mechanisms developed to cope with environmental unpredictability (Venable and Brown, 1988).

The separation into two phases with clear dominance of the escape-colonization strategy in the first phase and ultimate dominance of the protective-competition strategy in the second phase reflects short-term dominance of a colonization strategy at a restricted location such as the simulated site and long-term persistence of a superior competitor at this site. This agrees with the third expectation and with the predictions of the colonization-competition trade-off (Tilman, 1994; Cadotte, 2007). In support of these results, our own field observations showed that Malva parviflora (the escapecolonization strategy) presence in communities dominated by Anastatica hierochuntica (the protective-competition strategy) is usually sporadic and rarely observed during many subsequent 
years. These observations support the colonizer nature of Malva parviflora, which is of advantage in sites with high levels of disturbance or generally high unpredictability in the environmental conditions (Hutchinson, 1951; Hastings, 1980). However, it also highlights the dependence of this species on long-distance seed dispersal and on reintroductions to the communities by sheep or other animals (Michael et al., 2006).

Two overarching trends emerged across all scenarios: restricted overall community persistence and the clear separation of the community dynamics into two phases. The generality of these trends implies that stochastic processes might play a more important role than predefined environmental heterogeneity settings (Loik et al., 2004). The limited community persistence might point at the necessity to include more community processes in the model. For example, the model considers a community at a local spatial scale and does not include such large-scale processes as seed wash-in and wash-out (Hegazy and Kabiel, 2007) or occasional long-distance dispersal that might play a role for both species (Friedman and Stein, 1980; Michael et al., 2006). Thus, model performance and the representation of the strategies might be improved by enlarging the spatial extent of the simulated landscape. The other overarching trend, the two-phase nature of the simulation runs, reflects the performance of colonizers and competitors (Tilman, 1994; Calcagno et al., 2006).

Overall, this simulation study demonstrates the affinity of seed escape strategies to the colonization-competition trade-off. The connections between these two approaches appear more pronounced if seen through the lenses of a plant functional trait-approach, which facilitates their combination into more versatile plant survival strategies. Thus, we advocate for more comprehensive research on the combination of seed escape strategies and the colonization-competition trade-off in plant communities in deserts and beyond.

\section{Acknowledgements}

This research was funded by "Niedersächsisches Vorab" (MWK ZN3155) and Volkswagen Foundation. We are grateful to Nir Krintza from Ben-Gurion University of the Negev, Israel for providing the rainfall data and digital elevation models of the sites across the Negev Desert. We used the 'sequence-determines-credit' approach for author order. 


\title{
Chapter 4. Intra-specific trait variability in desert annual plant communities
}

This chapter is a manuscript to be submitted as a research article. The authors: L. Zakharova*, M. F. Arroyave Martinez*, K. M. Meyer, M. Seifan

* contributed equally

Authors' contribution:

M.S. and M.F.A.M. conceived of the presented idea.M.F.A.M. and M.S. designed and conducted the nethouse experiment. M.S. and M.F.A.M designed the method. L.Z. performed the analysis with a large input from K.M.M.'s and M.S. side. L.Z. led the writing of the manuscript with extensive input from M.F.A.M., K.M.M. and M.S..

\begin{abstract}
Desert plant communities are exposed to strong inter- and intraannual fluctuations in environmental factors. Interspecific or intraspecific trait variability provides survival of desert plants under these unstable conditions. In this research, we explored intraspecific trait variability and trait spaces of the desert annual plant Anastatica hierochutica. We focused on how the origin of environmentally diverse sites shapes the trait response of this species. The effects of aridity and soil salinity of the site of origin on plant adaptation to high salinity stress were analysed based on data from the nethouse experiment. We tested six hypotheses linking site characteristics with the changes in intraspecific trait spaces across five populations subjected to salinity treatments. The underlying analysis was based on functional traits such as dry weight, the number of leaves, seed germination probability, two gas exchange parameters and osmolyte concentration. Imposed high salinity had expected negative effects on two morphological traits. However, germination probability and osmolyte concentration were affected positively. Nevertheless, the influence of high salinity on the trait spaces of populations originating from the different sites was not equal. Under high salinity, the trait spaces of the stress-related trait of the populations from two relatively benign sites increased, whereas the trait spaces of the populations from the harsh environments decreased. The trait spaces of growth-related traits always decreased under high salinity regardless of the site of origin. While we were able to capture the effects of site char-
\end{abstract}


acteristics and their magnitude on the expression of the average value of functional traits, it was also shown that there is no one clear explanation of the changes in trait spaces as a response to salinity stress depending on the origin of the populations. However, there was a clear increase of the dissimilarity between the trait spaces of the populations originating from the same site but exposed to different salt stress levels with increasing environmental aridity. We advocate for outlining other factors determining environmental harshness and their interaction with each other. Additionally, collecting more information on interspecific variability might provide further insights into environmental filtering in these communities.

\subsection{Introduction}

Climate change will affect to some extent all the ecosystems across the globe (IPCC, 2018). Deserts are unique environments, which are characterized by strong intra- and interannual fluctuations in environmental factors (Biswas et al., 2016), and are specifically vulnerable to climate change and human impact (Loarie et al., 2009). The inborn variability in environmental conditions of desert ecosystems affects community structure, composition and dynamics (Loarie et al., 2009). Desert communities consist of not only different species but also of highly phenotypically diverse individuals. These variations among individuals influence intra- and inter-specific interactions and demographic parameters (Bolnick et al., 2011).

Trait-based approaches seem to be a suitable choice for desert communities as they can link morphological, physiological and phenological traits of individuals to species performance (Violle et al., 2007). Interspecific average trait values have been used as predictors for community dynamics for a long time (Mcgill et al., 2006; Albert et al., 2010), but intraspecific trait variability has recently also been acknowledged to play an important role in plant communities and was recommended to be included in both empirical and modelling studies (Burton et al., 2017). As individual response shapes communities (Violle et al., 2012), intraspecific trait variability is as important as interspecific trait variability when we aim to explain community structure and dynamics (Albert et al., 2017; Des Roches et al., 2018). Moreover, it has been shown to be insufficient to explain environmental filtering and niche 
differentiation based only on interspecific trait variability (Read et al., 2017) or to apply average trait values to describe niches (Warren and Lake, 2013). In communities with higher variability in trait values within rather than among species, considering of intraspecific trait variability is essential to predict the community structure (Read et al., 2017) as environmental filtering limits intraspecific trait variability. Additionally, explanation of such community-level processes as stabilizing niche differences and competitive asymmetries implies plasticity based on intraspecific trait variability (Turcotte and Levine, 2016). Incorporating intraspecific trait variability is especially meaningful when investigating population responses to environmental change (Jung et al., 2014) or studying communities with low diversity (Kraft et al., 2014) and poor species richness (Siefert et al., 2015). Since intraspecific trait variability may promote the resilience of a community, at least under small short-term environmental changes (Lepš et al., 2011), investigating desert community processes should take into account intraspecific trait variability.

Taking into account trait variability assists in predicting the outcomes of ecological interactions (Bolnick et al., 2011). However, the response of plants is defined not by a single trait, but by trait combinations and their trade-offs (Reich et al., 2003), so that the multiple traits approach should be applied for community ecology (Laughlin, 2014). Considering multiple trait combinations promotes understanding of trait adaptive values and, thus, facilitates prediction of species response to changes in the environment (Laughlin and Messier, 2015). Multidimensional trait space can be seen as the ecological niche of a species (Bonser, 2006) and provide necessary insights on community functional diversity (Mason et al., 2005).

As plants in deserts are subjected to strong environmental filtering (Biswas et al., 2016), increases of functional redundancy (after Carmona et al., 2016) between sites and decrease in species richness are expected. To increase the resilience of a community, narrowing of interspecific trait variability can be accompanied by increases in intraspecific trait variability (Pérez-Ramos et al., 2012; Jung et al., 2014). In this case, species loss would not decrease community functionality (Mayfield et al., 2010). As a result, species richness would be negatively correlated with intraspecific trait variability (Siefert et al., 2015). 
To test these assumptions on intraspecific trait variability and to measure corresponding changes in intraspecific trait spaces, we designed a nethouse experiment and measured a set of traits to capture the response of intraspecific trait space on the changes in main limiting factors in deserts such as aridity and salinity (Rewald et al., 2012; Gong et al., 2017).

We explore the response of desert plants from origins with different aridities, soil salinities and species richness to imposed salinity stress and investigate the role of intraspecific trait variability in these responses in line with recent claims (Moran et al., 2016). We selected populations of the desert annual plant Anastatica hierochuntica, which is known by its high tolerance to heat and high salinity (Eppel et al., 2014; Eshel et al., 2017). Anastatica hierochuntica grows across a soil water availability gradient in different dry ecosystems, from North Africa to Saudi Arabia (Gutterman, 1994). This species is characterized by its unique seed bank and seed dispersal strategy (Friedman et al., 1978; Kabiel, 2013), which is strongly correlated with the amount of rainfall (Hegazy and Kabiel, 2007). In the selected desert communities, A. hierochuntica is the dominating species, as it is expected for a stress-tolerant species that applies effective strategies to acquire limited resources (Pérez-Ramos et al., 2012; Copeland and Harrison, 2017).

With our experiment, we test the following hypotheses:

Hypothesis 1: Intraspecific trait variability in stress-related traits is decreasing along the natural aridity gradient from low to high aridity due to filtering, while intraspecific trait variability in traits related to growth and germination is increasing.

Hypothesis 2: Intraspecific trait variability is increasing with decreasing of community species richness because of interspecific competition, which leads to niche differentiation and separation.

Hypothesis 3: Intraspecific trait variability of growth traits is increasing with increasing of soil salinity of the site of origin, while intraspecific trait variability of stress-related traits is decreasing.

Hypothesis 4: High salinity has a positive effect on the average values of stress-related plant traits and a negative effect on the average values of plant traits not directly involved in stress response. 
Hypothesis 5: With increased imposed salt stress, intraspecific trait variability of growth traits is not changing, while intraspecific trait variability of stress-related traits is increasing. This response is more pronounced for plants originating from arid sites.

Hypothesis 6: The similarity of intraspecific trait spaces under no versus imposed salt stress is low and decreases with the involvement of stress-related traits.

\subsection{Methods}

\subsubsection{Research site and materials}

Research sites were located in the southern part of the Negev desert in Israel. This desert is characterized as a warm and dry desert with mean annual precipitation below $80 \mathrm{~mm}$. Seed material for the nethouse experiment was collected in five sites with different salinity, aridity and species richness (Tab. 4.1, Fig. 4.1). The aridity index was calculated according to De Martonne's method (Martonne, 1926) with low values representing high aridity of a site. The uniting characteristic of the sites was that all of them had a dominant and stable Anastatica hierochuntica population. We considered Anastatica hierochuntica as a suitable desert model plant for observations and experiments because of its inherited plasticity and supposed adaptation to harsh desert conditions.

Seeds from ten mother plants of Anastatica hierochuntica were collected in March 2016 from the five sites. The collected seeds were sown in February 2017, transplanted in March 2017, and harvested in the first week of June 2017, which resulted in 70 days of the experiment. An extra experiment after the end of the main one was conducted to define the germination probability of the secondgeneration seeds. Plants grew in 1-L pots containing a 1:1 mixture of soil and sand in a nethouse at Ben-Gurion University of the Negev. 
Table 4.1. Study sites and their characteristics used for the analysis. EC: soil electrical conductivity representing soil salinity (unpublished field measurements); AI: De Martonne aridity index with high values for low aridity (calculated from meteorological observations); SR: species richness expressed as the number of species found in the sites in 2016-2018.

\begin{tabular}{lllll}
\hline Site name & AI & SR & EC & Coordinates \\
\hline Timna & 0.62 & 27 & 1079.49 & $29.78,35$ \\
Uvda & 0.75 & 10 & 486.23 & $29.969,34.975$ \\
Shaharut & 0.75 & 2 & 2645.53 & $29.9,35$ \\
Meishar & 0.96 & 43 & 3795.75 & $30.41,34.94$ \\
Shefech Zohar & 1.11 & 6 & 6833.36 & $31.14,35.37$ \\
\hline
\end{tabular}

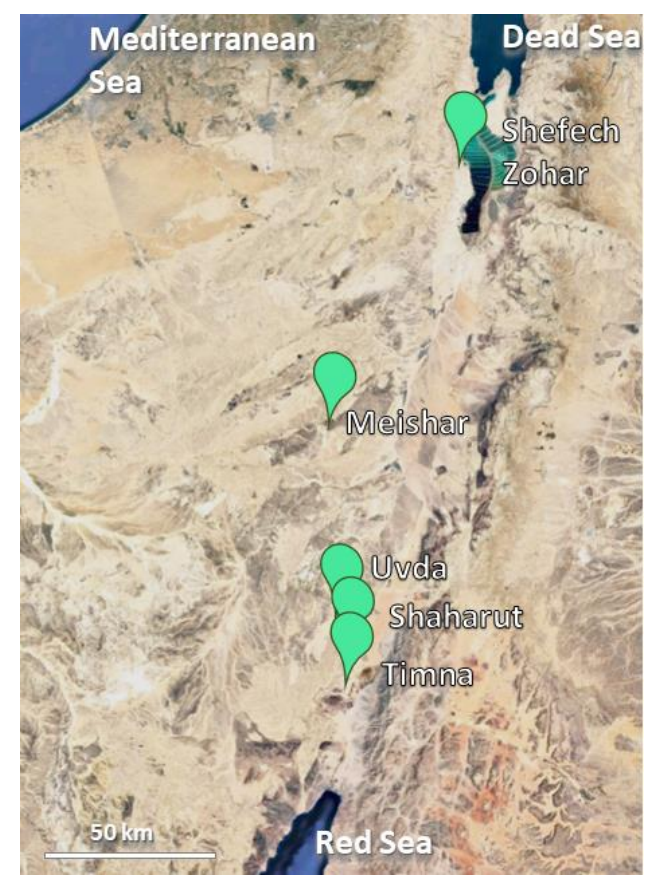

Figure 4.1. Sites of origin of the mother plants of the seeds in the experiment across the Negev Desert, Israel (c) Mapa GISrael, ORION-ME, 2020.

\subsubsection{Experimental design}

The experiment was set up to measure the expression of plant functional traits of Anastatica hierochuntica with and without exposure to high salinity. The plants in the experiment differed in their site of origin, which allowed us to conclude on intraspecific trait variability.

Ten individuals per site of origin were grown at two salt levels, control $(0 \mathrm{mM})$ and high (100 $\mathrm{mM})$. Salt stress experiments started on day 25 of the experiment, with $33 \%$ of the final salt solution. An increment of $33 \%$ in the salt concentrations was added on day 32 and day 39 of the experiment. 
Irrigation with salt was supplemented with $1 \mathrm{~g} \mathrm{L-1}$ of 20-20-20 NPK (Haifa Chemicals). The solution consisted of calcium chloride $\left(\mathrm{CaCl}_{2}\right)$ and sodium chloride $(\mathrm{NaCl})$ in a 1:1 relation. Both salts were added conjunctively to avoid a decrease in water infiltration, which could negatively influence root foraging due to sodium-induced dispersion, which reduces plant available water due to "surface crusting" and increased run-off (Rewald et al., 2012).

Ten seeds of each plant grown with and without salt solution were sown posterior to the main experiment in 1-L pots containing a 1:1 mixture of soil and sand in a nethouse at Ben-Gurion University of the Negev. This second experiment was conducted to determine germination probability of the second-generation seeds. Germination measurements were accomplished on day 30 of this new experiment.

Measurements of plant functional traits, such as the number of leaves and the gas exchange parameters photosynthetic rate $\left(\mathrm{A}_{\mathrm{N}}, \mu \mathrm{mol} \mathrm{CO}_{2} \mathrm{~m}^{-2} \mathrm{~s}^{-1}\right)$ and stomatal conductance $\left(\mathrm{g}_{\mathrm{s}}, \mathrm{mol} \mathrm{H}_{2} \mathrm{O} \mathrm{m}^{-2} \mathrm{~s}^{-1}\right)$ were conducted on day 60 of the experiment. Stomatal conductance $\left(g_{s}\right)$ and photosynthetic rate $\left[A_{N}\right.$ $\left.\left(\mu \mathrm{mol} \mathrm{CO}_{2} \mathrm{~m}^{-2} \mathrm{~s}^{-1}\right)\right]$ were obtained in parallel by exchange parameters using a portable LI-6400 Infrared Gas Analyzer (Li-Cor Inc., Lincoln, NE, USA). On day 70 of the experiment, the plants were harvested, and dry weight ( $\mathrm{g}$ ) of the entire plant body and osmolyte concentration in the leaf tissues $(\mathrm{mmol} / \mathrm{kg})$ were measured. Dry weight represented a morphological variable and was calculated by clipping living shoots at ground level and drying them for $48 \mathrm{~h}$ at $70^{\circ} \mathrm{C}$. Osmolyte accumulation in plant tissues is positively correlated with stressful environments such as drought, salinity, low temperature, and high irradiance. The osmolytes are compatible solutes that provide protection by decreasing electrolyte leakage (protecting the membrane integrity) and decreasing reactive oxygen species (Turrens, 2003) concentration, thus preventing oxidative damage in plants (Hayat et al., 2012). For osmolyte concentration measurements, fully expanded leaves were macerated in $\mathrm{N}_{2}$ liquid and placed into 2-ml Eppendorf tubes. After that, $10 \mu \mathrm{l}$ of supernatant was utilized to determine the osmolality using a vapour pressure osmometer (Vapro® 5520, Wescor, USA). Germination was estimated by collecting the seeds from each plant throughout the experiment. Developing fruits of each plant were covered with paper bags to prevent unintentional dispersal of seeds. After harvest, seeds from each 
plant were cleaned and stored in paper bags and tea bags with silica gel at $4{ }^{\circ} \mathrm{C}$ to avoid mould. To determine germination, seeds were directly placed in pots. Germination percentages were estimated on day 30 of the new experiment for ten seeds per individual.

\subsubsection{Statistical analysis}

\subsubsection{Generalized linear models to assess average trait responses}

To obtain a baseline impression of the responses of average trait values to site characteristics and salt treatment, we estimated the influence of the explanatory variables soil electrical conductivity, aridity index, and species richness of the site of origin as well as salt treatment on annual plant performance reflected in functional traits. Data analysis was done using R 3.5.1 (R Core Team, 2018).

We built one generalized linear model per response variable. Response variables were the following plant functional traits: dry weight of a plant, number of leaves, germination probability, gas exchange parameters photosynthetic rate $A_{N}$ and stomatal conductance $g_{s}$, and osmolyte concentration in leaves. For dry weight measurements, we applied generalized linear models with normally distributed errors and an identity link after a successful check of the respective assumptions. For gas exchange parameters $A_{N}$ and $g_{s}$ and osmolyte concentration in leaves, we built generalized linear models with Gamma distributed errors and an inverse link. We modelled the number of leaves with Poisson errors and $\log$ link and germination probability with binomial errors and logit link. Distribution family and link choice were based either on the nature of the data (number of leaves and germination probability) or on the Akaike information criterion (AIC) of the respective models and diagnostic plots from the R DHARMa package, version 0.2.5 (Hartig, 2019) to check the respective assumptions (for the other responses). Significance of model terms was assessed with F-tests except for the models with binomial or Poisson-distributed errors, where Chi-squared tests were used. In all models, we removed those interactions that produced singularities, but kept all other interactions, even if not significant, to preserve the original experimental design. 


\subsubsection{2. n-hypervolumes of functional trait space}

With n-hypervolumes we studied intraspecific trait variability of A. hierochuntica in the Negev Desert. We largely followed the protocol described in Bittebiere et al. (2019) and applied the R n-hypervolume package, version 2.0.11 (Blonder et al., 2014). n-hypervolumes are defined as a set of points within an $\mathrm{n}$-dimensional space. The axes of the $\mathrm{n}$-dimensional space represent biologically important and independent variables. Here, we chose plant functional traits for the axes. The number of these variables, which is equal to the number of the axes, defines the dimensionality of the n-hypervolumes. Values of these variables are a set of points forming a given n-hypervolume (Hutchinson, 1957; Blonder et al. 2014). n-hypervolume volumes are measured in SD-distances (SD for standard deviation) from the center, which is the grand mean of the respective functional trait over all related data points. Volumes of the n-hypervolume express the width of the multidimensional trait space of the species and thus intraspecific trait variability. Changes in n-hypervolumes can provide insights on the response of trait spaces to environmental changes. Overlaps of the n-hypervolumes, calculated as the multidimensional variation of Sorensen similarity, show the similarity of the compared multidimensional trait spaces, i.e. the similarity of trait values between the two sites or two treatments. High overlaps mean more similar spaces (Bittebierre et al. 2019).

Functional trait measurements for the n-hypervolume analysis were obtained in the nethouse experiment. We tested how stress through high salinity and the origin of a plant affected trait variability and plastic response. As characteristics of the site of origin, we considered species richness, soil electrical conductivity and aridity index (Tab. 4.1). For A. hierocuntica six functional traits were measured for individuals from five sites of origin.

Following Bittebiere et al. (2019), we first checked the six functional trait measurements for correlation. Since the distributions of all trait measurements except the dry weight were significantly different from normal distributions (Shapiro-Wilk tests, significance level 0.05), we determined nonparametric Kendall correlation coefficients. $A_{N}$ and $g_{s}(R=0.65)$ had a correlation coefficient greater than 0.6. Based on these results, we used only one trait measurement from this pair for n-hypervolume 
construction and volume calculation. Thus, five traits remained: dry weight, number of leaves, osmolyte concentration, germination probability, and gas exchange parameter $\mathrm{A}_{\mathrm{N}}$.

We centred and scaled trait data by using the mean and standard deviation of the data from all the treatments simultaneously in a standardization procedure, after Blonder et al. (2014) and Bittebier et al. (2019). This involved a z-transformation using the basic $\mathrm{R}$ function scale(). As a result, we obtained z-scores, which represent the precise location of each observation within a distribution of the values. To be able to calculate the volumes of the n-hypervolumes and compare them between each other, we excluded all NAs.

We constructed n-hypervolumes for both salt treatments, as well as for the different sites with the $\mathrm{R}$ n-hypervolume package. Each n-hypervolume was constructed by building a Gaussian kernel density estimate on an adaptive grid of random points wrapping around the original data points (Blonder et al., 2014). We calculated volumes of 3-, 4- and 5-trait hypervolumes to investigate how adding new traits affects the volume value. 3-trait hypervolumes included the morphological traits, such as dry weight and the number of leaves, and seed germination probability. These traits were measured for all individuals so that the corresponding hypervolumes were based on the maximum number of observations. To observe the effect of adding one dimension to a hypervolume, we added the gas exchange parameter $A_{N}$ for 4-trait hypervolumes. To investigate the effect of a stress-related trait on volumes, osmolyte concentration was added to produce 5-trait hypervolumes.

As the next step, we calculated the multidimensional variation of Sorensen similarity indices to estimate the similarity of n-hypervolumes constructed for two different salinity treatments or for two different sites of origin. Results of the calculation of n-hypervolumes were presented with the help of the R packages ggplot2 (Wickham, 2016) and dplyr, version 0.8.3 (Wickham et al., 2019). 


\subsection{Results}

\subsubsection{Generalized linear models to assess average trait responses}

High soil salt concentration had a significant negative effect on the plant functional traits dry weight and number of leaves (Tab. 4.2 and 4.3, Appendix. Tab. S4.1). However, the effect of high salinity on germination probability and osmolyte concentration was significantly positive (Tab. 4.4 and 4.5, Appendix. Tab. S4.1), and there were no significant effects of high salinity on any of the gas exchange parameters (Tab. 4.6 and 4.7). The aridity index of the site of origin had a significant positive effect on dry weight and the number of leaves, as well as a significant negative effect on germination probability and both gas exchange parameters (Tab. 4.2 - 4.4, 4.6 and 4.7, Appendix. Tab. S4.1). The only significant effect of soil electrical conductivity of the site of origin was a positive effect on the number of leaves (Tab. 4.3, Appendix. Tab. S4.1). None of the plant functional traits showed a significant response to species richness as a main effect. For dry weight and the number of leaves, the interaction between soil electrical conductivity and aridity index of the site of origin had a small significant negative effect (Tab. 4.2 and 4.3, Appendix. Tab. S4.1). For all other plant functional traits, this interaction effect was small and significantly positive (Tab. $4.4-4.7$, Appendix. Tab. S4.1). The number of leaves was the only plant trait that showed further significant interaction effects, i.e. small positive effects of the interactions between species richness of the site of origin and salt treatment as well as soil electrical conductivity of the site of origin and salt treatment (Tab. 4.3, Appendix. Tab. S4.1). 
Table 4.2. Analysis of deviance table with the significance of each predictor for dry weight as a response variable. EC: soil electrical conductivity of the site of origin; AI: De Martonne aridity index, SR: species richness, HS: treatment with adding salt solution (high salinity). EC x AI, EC x HS, AI x HS, SR x HS, and EC x AI x HS are interactions between the corresponding factors. Significant values are in bold fonts $(\mathrm{p}<0.05)$.

\begin{tabular}{lllllll}
\hline Explanatory variable & Df & Deviance & Resid. Df & Resid. Dev & F & Pr $(>\mathbf{F})$ \\
\hline Intercept & & & 83 & 75,339 & & \\
EC & 1 & 1.492 & 82 & 73.847 & 2.609 & 0.111 \\
AI & $\mathbf{1}$ & $\mathbf{2 . 3 5 1}$ & $\mathbf{8 1}$ & $\mathbf{7 1 . 4 9 6}$ & $\mathbf{4 . 1 1}$ & $\mathbf{0 . 0 4 6}$ \\
SR & 1 & 0.401 & 80 & 71.095 & 0.701 & 0.405 \\
HS & $\mathbf{1}$ & $\mathbf{2 3 . 0 8}$ & $\mathbf{7 9}$ & $\mathbf{4 8 . 0 1 5}$ & $\mathbf{4 0 . 3 5 2}$ & $\mathbf{1 . 5 E - 0 8}$ \\
EC x AI & $\mathbf{1}$ & $\mathbf{4 . 5 6 8}$ & $\mathbf{7 8}$ & $\mathbf{4 3 . 4 4 7}$ & $\mathbf{7 . 9 8 7}$ & $\mathbf{0 . 0 0 6}$ \\
EC x HS & 1 & 0.019 & 77 & 43.428 & 0.033 & 0.855 \\
AI x HS & 1 & 0.095 & 76 & 43.333 & 0.165 & 0.685 \\
SR x HS & 1 & 1.007 & 75 & 42.326 & 1.761 & 0.189 \\
EC x AI x HS & 1 & 0.001 & 74 & 42.325 & 0.002 & 0.966 \\
\hline
\end{tabular}

Table 4.3. Analysis of deviance table with the significance of each predictor for number of leaves as a response variable. EC: soil electrical conductivity of the site of origin; AI: De Martonne aridity index, SR: species richness, HS: treatment with adding salt solution (high salinity). EC x AI, EC x HS, AI x HS, SR x HS, and EC x AI x HS are interactions between the corresponding factors. Significant values are in bold fonts $(\mathrm{p}<0.05)$.

\begin{tabular}{llllll}
\hline Explanatory variable & Df & Deviance & $\begin{array}{l}\text { Resid. } \\
\text { Df }\end{array}$ & Resid. Dev & Pr( $>$ Chi) \\
\hline Intercept & & & 83 & 1950.39 & \\
EC & $\mathbf{1}$ & $\mathbf{2 9 . 8 6 5}$ & $\mathbf{8 2}$ & $\mathbf{1 9 2 0 . 5 2}$ & $\mathbf{4 . 6 3 E - 0 8}$ \\
AI & $\mathbf{1}$ & $\mathbf{4 1 . 5 1}$ & $\mathbf{8 1}$ & $\mathbf{1 8 7 9 . 0 1}$ & $\mathbf{1 . 1 7 E - 1 0}$ \\
SR & 1 & 1.204 & 80 & 1877.8 & 0.272 \\
HS & $\mathbf{1}$ & $\mathbf{1 3 1 3 . 8 8}$ & $\mathbf{7 9}$ & $\mathbf{5 6 3 . 9 2 3}$ & $\mathbf{1 . 1 E}-287$ \\
EC x AI & $\mathbf{1}$ & $\mathbf{1 8 . 7 8}$ & $\mathbf{7 8}$ & $\mathbf{5 4 5 . 1 3 9}$ & $\mathbf{1 . 5 E - 0 5}$ \\
EC x HS & $\mathbf{1}$ & $\mathbf{3 . 9 9}$ & $\mathbf{7 7}$ & $\mathbf{5 4 1 . 1 4 8}$ & $\mathbf{0 . 0 4 6}$ \\
AI x HS & 1 & 0.027 & 76 & 541.12 & 0.869 \\
SR x HS & $\mathbf{1}$ & $\mathbf{7 . 0 7 7}$ & $\mathbf{7 5}$ & $\mathbf{5 3 4 . 0 4 4}$ & $\mathbf{0 . 0 0 8}$ \\
EC x AI x HS & 1 & 2.671 & 74 & 531.374 & 0.1028 \\
\hline
\end{tabular}


Table 4.4. Analysis of deviance table with the significance of each predictor for seed germination probability as a response variable. EC: soil electrical conductivity of the site of origin; AI: De Martonne aridity index, SR: species richness, HS: treatment with adding salt solution (high salinity). EC x AI, EC x HS, AI x HS, SR x HS, and EC x AI x HS are interactions between the corresponding factors. Significant values are in bold fonts $(\mathrm{p}<0.05)$.

\begin{tabular}{llllll}
\hline Explanatory variable & Df & Deviance & Resid. Df & Resid. Dev & Pr(>Chi) \\
\hline Intercept & & & 83 & 319.841 & \\
EC & 1 & 0.586 & 82 & 319.255 & 0.444 \\
AI & $\mathbf{1}$ & $\mathbf{6 . 6 2 8}$ & $\mathbf{8 1}$ & $\mathbf{3 1 2 . 6 2 6}$ & $\mathbf{0 . 0 1}$ \\
SR & 1 & 3.147 & 80 & 309.479 & 0.076 \\
HS & $\mathbf{1}$ & $\mathbf{3 6 . 7 6}$ & $\mathbf{7 9}$ & $\mathbf{2 7 2 . 7 1 8}$ & $\mathbf{1 . 3 4 E - 0 9}$ \\
EC x AI & $\mathbf{1}$ & $\mathbf{6 . 5 8 2}$ & $\mathbf{7 8}$ & $\mathbf{2 6 6 . 1 3 5}$ & $\mathbf{0 . 0 1}$ \\
EC x HS & 1 & 2.93 & 77 & 263.204 & 0.087 \\
AI x HS & 1 & 0.003 & 76 & 263.201 & 0.954 \\
SR x HS & 1 & 0.086 & 75 & 263.115 & 0.769 \\
EC x AI x HS & 1 & 2.793 & 74 & 260.322 & 0.095 \\
\hline
\end{tabular}

Table 4.5. Analysis of deviance table with the significance of each predictor for osmolyte concentration as a response variable. EC: soil electrical conductivity of the site of origin; AI: De Martonne aridity index, SR: species richness, HS: treatment with adding salt solution (high salinity). EC x AI, EC x HS, AI x HS, SR x HS, and EC x AI x HS are interactions between the corresponding factors. Significant values are in bold fonts $(\mathrm{p}<0.05)$.

\begin{tabular}{lllllll}
\hline Explanatory variable & Df & Deviance & Resid. Df & Resid. Dev & F & Pr(>F) \\
\hline Intercept & & & 51 & 19.841 & & \\
EC & 1 & 0.021 & 50 & 19.82 & 1 & 0.323 \\
AI & 1 & 0.003 & 49 & 19.817 & 0.152 & 0.698 \\
SR & 1 & 0.014 & 48 & 19.803 & 0.672 & 0.417 \\
HS & $\mathbf{1}$ & $\mathbf{1 8 . 8 8 5}$ & $\mathbf{4 7}$ & $\mathbf{0 . 9 1 8}$ & $\mathbf{9 0 6 . 7 6}$ & $\mathbf{4 . 6 2 E - 3 0}$ \\
EC x AI & 1 & 0.01 & 46 & 0.908 & 0.488 & 0.489 \\
EC x HS & 1 & 0.025 & 45 & 0.883 & 1.188 & 0.282 \\
AI x HS & 1 & 0.016 & 44 & 0.866 & 0.787 & 0.38 \\
SR x HS & 1 & 0.004 & 43 & 0.862 & 0.196 & 0.66 \\
EC x AI x HS & 1 & 0.0004 & 42 & 0.862 & 0.02 & 0.887 \\
\hline
\end{tabular}


Table 4.6. Analysis of deviance table with the significance of each predictor for stomatal conductance $\mathrm{g}_{\mathrm{s}}$ as a response variable. EC: soil electrical conductivity of the site of origin; AI: De Martonne aridity index, SR: species richness, HS: treatment with adding salt solution (high salinity). EC x AI, EC x HS, AI x HS, SR x HS, and EC x AI x HS are interactions between the corresponding factors. Significant values are in bold fonts $(\mathrm{p}<0.05)$.

\begin{tabular}{lllllll}
\hline Explanatory variable & Df & Deviance & Resid. Df & Resid. Dev & F & Pr(>F) \\
\hline Intercept & & & 59 & 15.293 & & \\
EC & 1 & 0.025 & 58 & 15.269 & 0.118 & 0.733 \\
AI & $\mathbf{1}$ & $\mathbf{1 . 4 1 3}$ & $\mathbf{5 7}$ & $\mathbf{1 3 . 8 5 6}$ & $\mathbf{6 . 5 7}$ & $\mathbf{0 . 0 1 3}$ \\
SR & 1 & 0.002 & 56 & 13.853 & 0.011 & 0.918 \\
HS & 1 & 0.497 & 55 & 13.357 & 2.311 & 0.135 \\
EC x AI & $\mathbf{1}$ & $\mathbf{2 . 2 4}$ & $\mathbf{5 4}$ & $\mathbf{1 1 . 1 1 6}$ & $\mathbf{1 0 . 4 1 9}$ & $\mathbf{0 . 0 0 2}$ \\
EC x HS & 1 & 0.241 & 53 & 10.875 & 1.122 & 0.295 \\
AI x HS & 1 & 0.113 & 52 & 10.762 & 0.526 & 0.472 \\
SR x HS & 1 & 0.041 & 51 & 10.721 & 0.192 & 0.663 \\
EC x AI x HS & 1 & 0.014 & 50 & 10.706 & 0.066 & 0.799 \\
\hline
\end{tabular}

Table 4.7. Analysis of deviance table with the significance of each predictor for photosynthetic rate $A_{N}$ as a response variable. EC: soil electrical conductivity of the site of origin; AI: De Martonne aridity index, SR: species richness, HS: treatment with adding salt solution (high salinity). EC x AI, EC x HS, AI x HS, SR x HS, and EC x AI x HS are interactions between the corresponding factors. Significant values are in bold fonts $(\mathrm{p}<0.05)$.

\begin{tabular}{lllllll}
\hline Explanatory variable & Df & Deviance & Resid. Df & Resid. Dev & F & Pr(>F) \\
\hline Intercept & & & 59 & 18.982 & & \\
EC & 1 & 0.339 & 58 & 18.644 & 1.35 & 0.251 \\
AI & $\mathbf{1}$ & $\mathbf{1 . 7 7}$ & $\mathbf{5 7}$ & $\mathbf{1 6 . 8 7 4}$ & $\mathbf{7 . 0 5}$ & $\mathbf{0 . 0 1 1}$ \\
SR & 1 & 0.218 & 56 & 16.656 & 0.868 & 0.356 \\
HS & 1 & 0.871 & 55 & 15.784 & 3.472 & 0.068 \\
EC x AI & $\mathbf{1}$ & $\mathbf{1 . 3 6 8}$ & $\mathbf{5 4}$ & $\mathbf{1 4 . 4 1 6}$ & $\mathbf{5 . 4 4 9}$ & $\mathbf{0 . 0 2 4}$ \\
EC x HS & 1 & 0.273 & 53 & 14.143 & 1.089 & 0.302 \\
AI x HS & 1 & 0.305 & 52 & 13.838 & 1.215 & 0.276 \\
SR x HS & 1 & 0.009 & 51 & 13.828 & 0.039 & 0.845 \\
EC x AI x HS & 1 & 0.004 & 50 & 13.825 & 0.015 & 0.903 \\
\hline
\end{tabular}

\subsection{2. n-hypervolumes of functional trait space}

As a preparation for the n-hypervolume analyses, we found that photosynthetic gas exchange parameters $\left(A_{N}\right.$ and $\left.g_{s}\right)$ were highly correlated with each other $(R=0.63)$, but not with any of the other traits. As expected, dry weight and number of leaves also had a relatively high correlation $(R=0.49)$. Osmolyte concentration had a high negative correlation with the number of leaves $(\mathrm{R}=-0.55)$ and dry weight $(\mathrm{R}=-0.28)$. Germination probability of seeds was negatively correlated with dry weight $(\mathrm{R}=-$ 


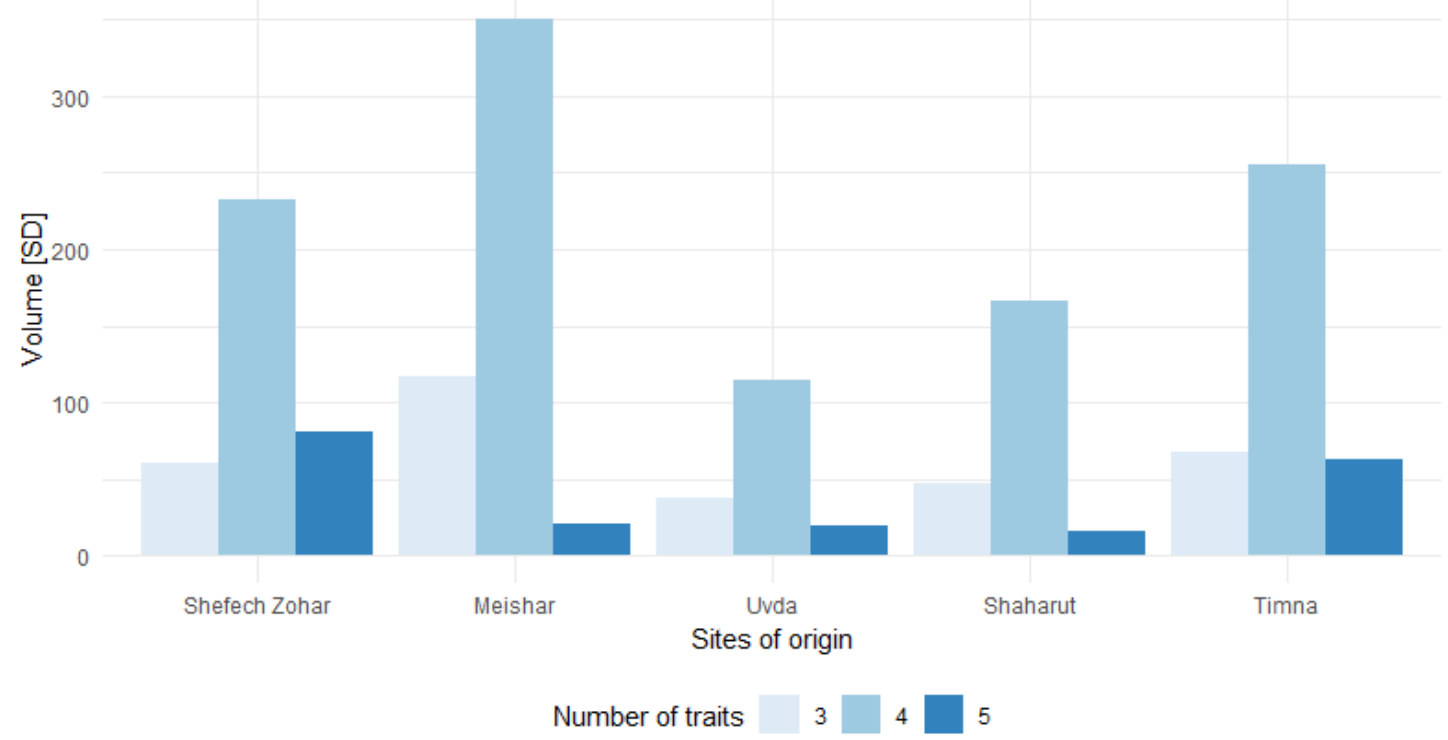

Figure 4.2. Volumes of hypervolumes as proxies of trait spaces constructed for the populations under the control salinity treatment for all the sites of origin based on three, four and five traits. Three traits include only morphological traits and seed germination probability, four traits include morphological traits plus a gas exchange parameter and five traits add osmolyte concentration as a stress-related trait. The sites are ordered based on their aridity from the least arid to the most arid site (from the largest aridity index AI to the smallest).

0.19) and the number of leaves $(\mathrm{R}=-0.18)$. Following the recommendations from Bittebiere et al. (2019), we excluded one of the traits involved in a correlation with a coefficient larger than 0.6 (gas exchange parameter $g_{s}$ ) and continued the analysis with the remaining five traits.

Under the control salinity treatment, the volumes of hypervolumes increased after adding the fourth trait, gas exchange parameter $\mathrm{A}_{\mathrm{N}}$ and decreased after adding the fifth, osmolyte concentration

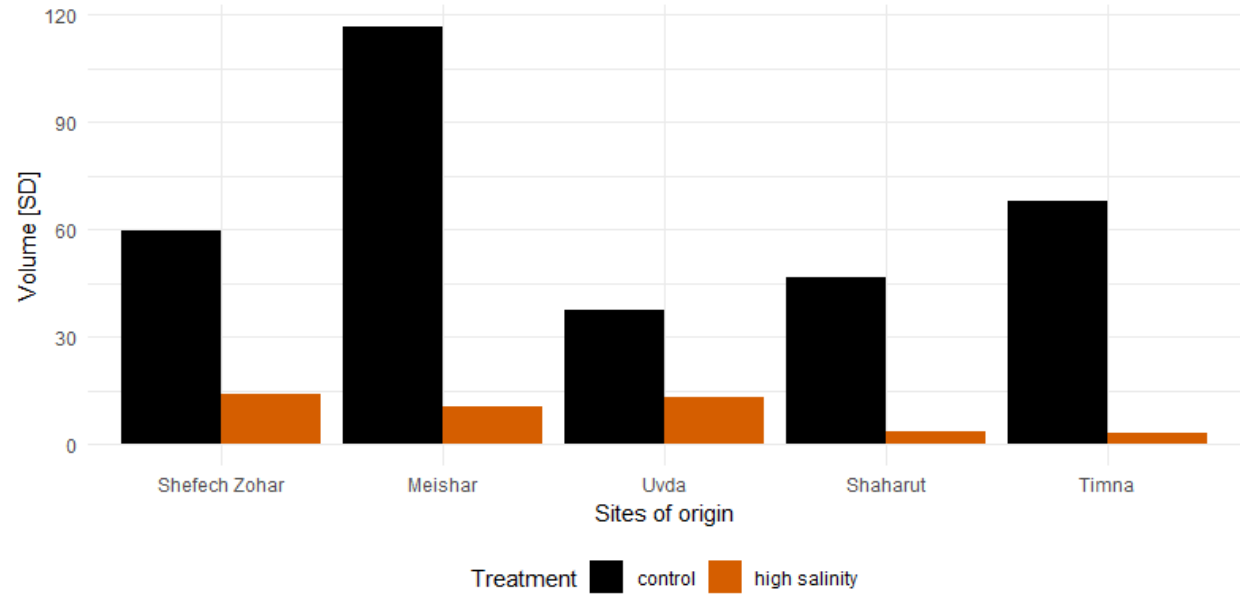

Figure 4.3. Volumes of hypervolumes as proxies of trait spaces constructed from three traits for the populations under the control salinity treatment vs. high salinity treatment. Three traits include only morphological traits and seed germination probability, four traits include morphological traits plus a gas exchange parameter and five traits add osmolyte concentration as a stress-related trait. The sites are ordered based on their aridity from the least arid to the most arid site (from the largest aridity index AI to the smallest). 
(Fig. 4.2). For all sites of origin but for Shefech Zohar, the volumes of 5-trait hypervolumes were, as a result, smaller than for 3-trait hypervolumes. The volumes of 5-trait hypervolumes decreased from the least to the more arid sites except for Timna, the most arid site. The volumes of 4-trait and 3-trait hypervolumes increased from Shefech Zohar to Meishar and from Uvda and Timna.

The population from Meishar, the site with the highest species richness, had the smallest 3trait and 4-trait hypervolumes, whereas the population from Shaharut, where A. hierochuntica is accompanied only by one more species, had one of the smallest 3-trait and 4-trait hypervolumes (Fig. 4.2). Furthermore, the population from Shefech Zohar had the largest 5-trait hypervolumes, while the population from Shaharut had the smallest 5-trait hypervolume. The population from Uvda, the site with lowest soil salinity, had the smallest 3- and 4-trait hypervolumes (Fig. 4.2). 


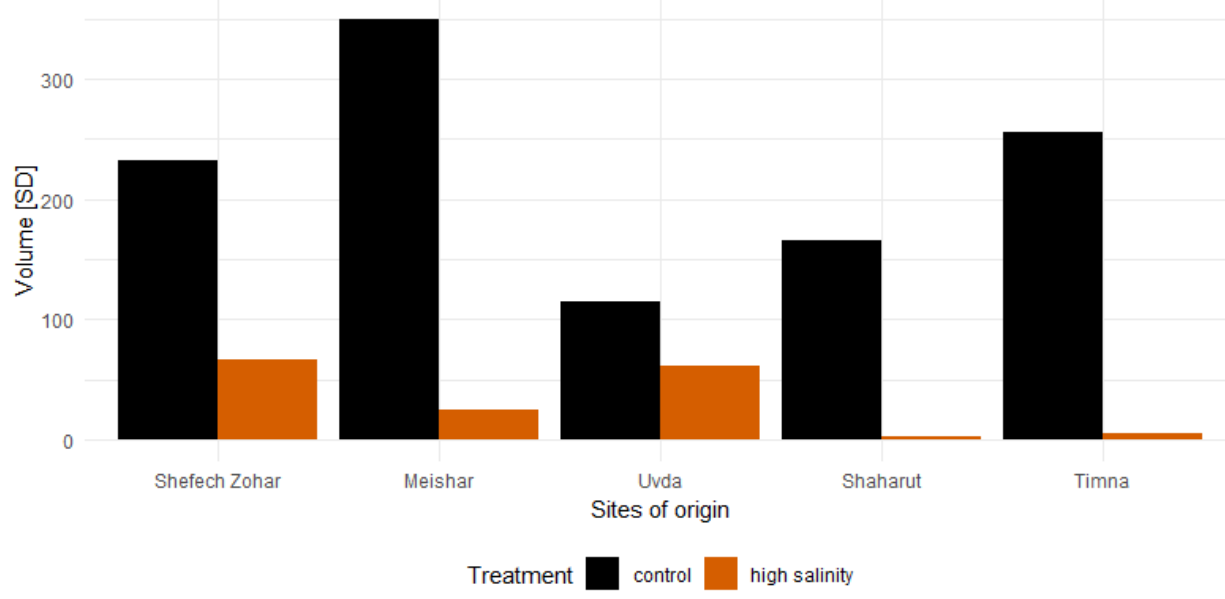

Figure 4.4. Volumes of hypervolumes as proxies of trait spaces constructed from four traits for the populations under the control salinity treatment vs. high salinity treatment. Three traits include only morphological traits and seed germination probability, four traits include morphological traits plus a gas exchange parameter and five traits add osmolyte concentration as a stress-related trait. The sites are ordered based on their aridity from the least arid to the most arid site (from the largest aridity index AI to the smallest).

For 3- and 4-trait hypervolumes, the high salinity treatment caused a reduction of the trait space of the corresponding populations (Fig. 4.3 and 4.4). High salinity in the 5-trait hypervolumes led to the increase in the volumes of the trait spaces for the populations originating from Uvda and Meishar (Fig. 4.5). However, this increase of the stress-related trait space under high salinity was not depended on the aridity of the site of origin (Fig. 4.5).

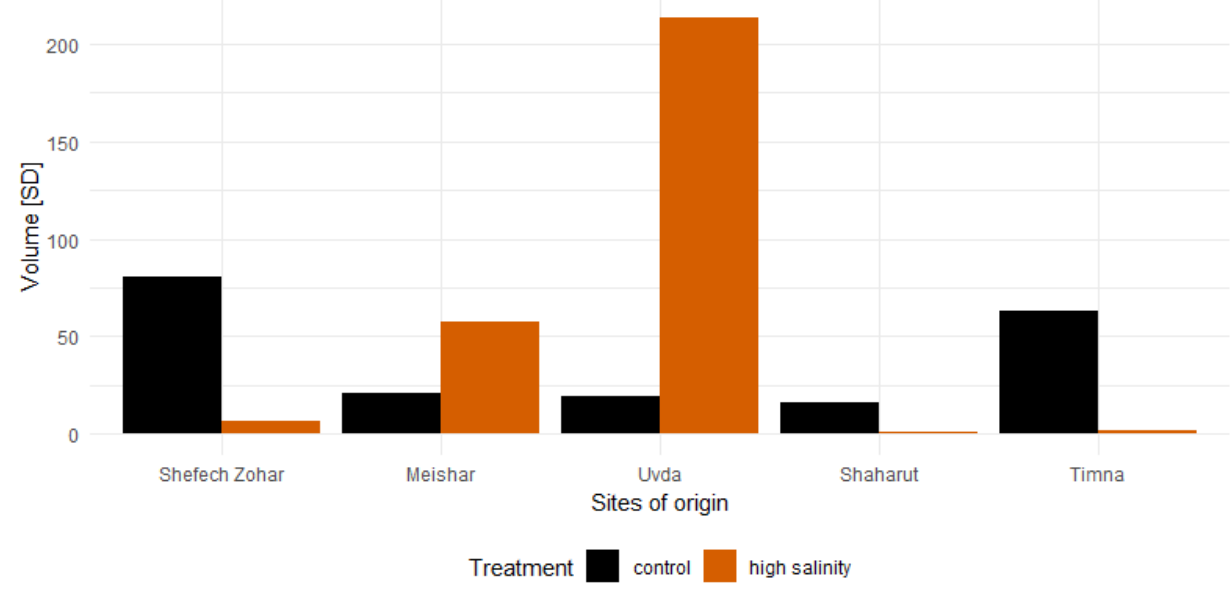

Figure 4.5. Volumes of hypervolumes as proxies of trait spaces constructed from five traits for the populations under the control salinity treatment vs. high salinity treatment. Three traits include only morphological traits and seed germination probability, four traits include morphological traits plus a gas exchange parameter and five traits add osmolyte concentration as a stress-related trait. The sites are ordered based on their aridity from the least arid to the most arid site (from the largest aridity index AI to the smallest). 
Table 4.8. The similarity between the trait spaces of populations from the same site of origin that were exposed to the high salinity treatment or the control treatment. The similarity was calculated as the Sorensen similarity index between the corresponding n-hypervolumes, where zero shows no overlap, i.e. the trait spaces are dissimilar, and one shows the complete identity of the trait spaces.

\begin{tabular}{llll}
\hline & 3 traits & 4 traits & 5 traits \\
\hline General & 0.193 & 0.117 & 0 \\
Meishar & 0.143 & 0.083 & 0 \\
Shaharut & 0.038 & 0.013 & 0 \\
Shefech Zohar & 0.14 & 0.116 & 0 \\
Timna & 0.072 & 0.024 & 0 \\
Uvda & 0.118 & 0.126 & 0.008 \\
\hline
\end{tabular}

The similarity between the hypervolumes from the same population under control versus high salinity treatment was generally low and decreased with the increase of the number of traits included in the hypervolume calculation, and especially with the involvement of the stress-related trait (Tab. 4.8). For example, the only site for which any overlaps at all were found for 5-trait hypervolumes was Uvda, the most benign site with respect to soil salinity. Populations from the sites with higher soil salinity showed overlapping hypervolumes between the control and high salinity treatment only for 3and 4-trait hypervolumes, which included only growth traits. Here, the most stressed sites with respect to aridity, Timna and Shaharut, showed the smallest overlap of control- versus high-salinity hypervolumes.

The same tendency was found for the similarity between hypervolumes from different populations under the same salinity treatment: the similarity decreased with the increase of the traits included in the hypervolume calculation only with few deviations from this tendency (Appendix. Tab. A4.2).

\subsection{Discussion}

In this study, we tested six hypotheses on how environment filtering shapes intraspecific trait space and how high salinity stress affects intraspecific trait variability spaces of the annual species A. hierochuntica. Based on six functional traits, we compared populations from five sites located on the northsouth gradient of the desert, which differed from each other in their salinity, aridity and species rich- 
ness. We compared calculated hypervolumes between the populations of A. hierochuntica originating from different sites and between two salt treatments for plants from the same population. Additionally, we observed how the trait space volumes changed when we included traits stepwise. The initial 3-trait hypervolumes included only morphological traits and seed germination probability, while the 4-trait hypervolumes included a gas exchange parameter, and the final 5-trait hypervolumes also included osmolyte concentration as a salt-stress related trait.

Intraspecific trait variability of the stress-related trait in our analysis, osmolyte concentration, was smaller than for other traits. This indicates that A. hierochuntica is less plastic in the trait of osmolyte concentration than in other traits. These results contradict expectations that physiological traits are more plastic than morphological traits (DeWitt et al., 1998; Valladares et al., 2000). One possible explanation might be that the effect of the abiotic conditions was lower than expected for this species (Jung et al., 2014). However, we were able to support our Hypothesis 1 only partially: stress-related trait spaces showed indeed smaller trait spaces at high aridity with an exception for the population from the most arid site at Timna, but the growth trait-space increased with aridity only within two distinct groups of sites. These results imply that the aridity of the site of origin is not the only factor defining the size of the trait spaces, and the environments might be stressed based on other criteria. Moreover, trait values may often have small average values in stressed environments (as it was the case in this study, see results relating to Hypothesis 4), so that the expected increase in intraspecific trait variability cannot be achieved due to the relative proximity of the average trait values to zero.

Intraspecific trait variability was not increasing with the decrease of community species richness as was originally expected by Hypothesis 2 . This hypothesis was based on an idea that increasing intraspecific trait variability would compensate the decrease in interspecific trait variability and species richness loss (Pérez-Ramos et al., 2012; Jung et al., 2014; Siefert et al., 2015) as a result of strong environmental filtering (Biswas et al., 2016). Thus, additional information on corresponding interspecific trait variability is needed to draw a conclusion on niche differentiation processes in these desert communities (Turcotte and Levine, 2016; Read et al., 2017). 
Our analysis results also were in disagreement with Hypothesis 3 that the populations originating from high salinity sites have larger trait spaces of growth traits and smaller trait spaces of stressrelated traits. This hypothesis was in line with the ideas that under unfavourable conditions the genetic variability would increase for the traits not involved in stress response directly (Hoffmann and Merilä, 1999). In benign sites, the trait space of growth-related traits was small, as well, supporting the idea of higher stability of this group of traits relative to physiological stress-related traits (Kazakou et al., 2014; Siefert et al., 2015).

Average trait value responses largely supported Hypothesis 4. As expected, high salinity had a positive effect on the stress-related plant trait osmolyte concentration and a negative effect on dry weight and number of leaves, which are morphological traits that are not directly involved in the stress response. This reinforces earlier reports on negative effects of salinity on average plant performance (Ahmed et al., 2013) and may indicate that stronger environmental filtering decreases not only intraspecific trait-variability but also average trait values (Spasojevic and Suding, 2012). In slight disagreement with hypothesis 4 , other non-stress related traits responded positively to high salinity (germination probability) or not at all (gas exchange parameters). The positive response of germination probability to high aridity of the site of origin of the mother plants was also in contrast to the expected negative responses of dry weight and number of leaves. This may imply that under stressful conditions such as high aridity of the origin and high current salinity, plants follow the strategy to invest more in long-term survival over generations than in an instantaneous increase of the biomass in a specific stress year. In this case, germination is driven by the environments to which both mother plants and seeds were exposed (Gutterman, 2000). As the growing conditions for seeds were benign in this experiment, the positive effect of high salinity on germination of the next generation might also be explained by the influence of the environment of mother plants (Uller, 2008) and the general adaptation of A. hierochuntica to high salinity.

Moreover, our results directly disagreed with the first and second part of Hypothesis 5. Neither did the trait space of growth traits remain unchanged, nor did the trait space of stress-related traits increase under high salinity for the populations originating from more arid or otherwise stressed sites. 
The trait space of stress-related traits under the high salinity treatment increased only for relatively benign sites. These results can imply that the populations from relatively benign environments might invest more resources in the variability of stress-related traits in case of stress, while the populations from harsh environments demonstrate low variability in stress-related traits. This contradiction to our expectations based on Liancourt and Tielbörger (2009) and Castro et al. (2013) might lie in the nature of this specific stress-related trait. Higher osmolyte concentration ensures the protection of plant cells from the damaging effect of salinity stress (Hayat et al., 2012). Thus, it is expected that the plants originating from more harsh environments have a better adaptation to the respective stress and respond to the stress in an appropriate way, while plants from benign conditions should still seek for better response and show larger trait variability.

Similarity assessments between the trait spaces of populations originating from different sites or between the populations from the same site but subjected to different salinity treatments revealed how trait spaces depended on the environment. In general, the similarity between two sites decreased with the increase of the number of traits, which can be explained by gradually introducing additional trait variation associated with a new trait. However, this result might be also biased by our choice of the traits: we started n-hypervolume calculation with morphological traits that were shown to be more conservative (Kazakou et al., 2014; Siefert et al., 2015). In line with Hypothesis 6, the dissimilarities between the populations from the sites under different salinity treatments were so large that the overlap in trait spaces built on all five traits was zero for all sites but one, the site with the lowest soil salinity. These results highlight a possible lack of plasticity in salt-related stress traits for the populations originating from benign sites causing a decrease in adaptation to high salinity. Except for such populations, these results are also in line with the statement by Bittebiere et al. (2019) that trait spaces of the populations under benign conditions have a small overlap with those under stress.

Overall, we observed high intraspecific trait variability in A. hierochuntica. This variability partially can be explained by environmental heterogeneity across the sites of origin. Within-site heterogeneity of environmental conditions was not part of this study but may be interesting to explore in future studies to potentially extend this explanation to finer spatial scales. The key factors determining 
intraspecific trait variability in our study seemed to be aridity and soil salinity. The effect of species richness, which was used as an additional site characteristic, should be explored more, e.g. we advocate for including interspecific trait variability in the analysis to compare A. hierochuntica with other species growing in its communities. Additionally, to better capture multidimensional trait space (Bittebiere et al., 2019), further traits might have to be included in the analysis of species richness effects. The intensity of the influence of the aridity and soil salinity depends on the nature of the stress to which an individual was exposed at the moment of observation. In our experiment, we considered salt stress, and, consequently, this treatment increased the role of the soil salinity of the site of the origin. However, salt stress differentially affected trait expression, e.g. with respect to dry weight and number of leaves on the one side and germination probability and osmolyte concentration on the other side. This complicates the interpretation of salt stress on plant performance and survival. Thus, the decision on which traits should be included in consideration of trait spaces for a specific experiment or field observation should be made carefully. We also advise conducting further investigations of the relationships between functional traits, their variability, and the environment they are expressed in, with a particular focus on intraspecific trait variability. Such investigations will help to further unravel the key role that intraspecific trait variability plays for the success and stability of annual plant communities in the ever-changing environment of warm deserts.

\section{Acknowledgements}

The implementation of the experiment was supported by the Koshland Foundation and would not have been possible without the technical assistance of all the technicians at the Sde Boker campus of BenGurion University of the Negev. We are grateful to Nir Krintza from Ben-Gurion University, Israel, for providing information about the research sites. Work on this manuscript was funded by "Niedersächsisches Vorab" (MWK ZN3155) and Volkswagen Foundation. 


\title{
Chapter 5. Metamodels for evaluating, calibrating and applying agent-based models: A review
}

This chapter was published as: Pietzsch, B., Fiedler, S., Mertens, K. G., Richter, M., Scherer C., Widyastuti, K., Wimmler, M. C., Zakharova L. \& Berger, U. (2020). Metamodels for Evaluating, Calibrating and Applying Agent-Based Models: A Review. Journal of Artificial Societies and Social Simulation, 23(2), 1-9. https://doi.org10.18564/jasss.4274.

Authors' contribution: B.P. and U.B. conceived of the presented idea. B.P. and U.B. designed and conducted the literature review. All authors designed the rating method. B.P. performed the analysis with a large input from L.Z.'s side. B.P. led the writing of the manuscript with extensive input from all other authors.

Appendices are available at: http://jasss.soc.surrey.ac.uk/23/2/9.html.

\begin{abstract}
The recent advancement of agent-based modeling is characterized by higher demands on the parameterization, evaluation and documentation of these computationally expensive models. Accordingly, there is also a growing request for "easy to go" applications just mimicking the input-output behavior of such models. Metamodels are being increasingly used for these tasks. In this paper, we provide an overview of common metamodel types and the purposes of their usage in an agent-based modeling context. To guide modelers in the selection and application of metamodels for their own needs, we further assessed their implementation effort and performance. We performed a literature research in January 2019 using four different databases. Five different terms paraphrasing metamodels (approximation, emulator, meta-model, metamodel and surrogate) were used to capture the whole range of relevant literature in all disciplines. All metamodel applications found were then categorized into specific metamodel types and rated by different junior and senior researches from varying disciplines (including forest sciences, landscape ecology, or economics) regarding the implementation effort and performance. Specifically, we captured the metamodel performance according to (i) the consideration of uncertainties, (ii) the suitability assessment provided by the authors for the particular purpose, and (iii)
\end{abstract}


the number of valuation criteria provided for suitability assessment. We selected 40 distinct metamodel applications from studies published in peer-reviewed journals from 2005 to 2019 . These were used for the sensitivity analysis, calibration and upscaling of agent-based models, as well to mimic their prediction for different scenarios. This review provides information about the most applicable metamodel types for each purpose and forms a first guidance for the implementation and validation of metamodels for agent-based models.

\subsection{Introduction}

Essentially, a metamodel (MM) is a model that describes the behavior of an original model on a higher hierarchical level (Moorcroft et al., 2001; Urban, 2005; Gore et al., 2017). In the context of mechanistically detailed and therefore often computationally expensive agent-based models (ABM) or individual-based models $\left(\mathrm{IBM}^{1}\right)$, MMs provide an efficient way to facilitate profound model analysis and prediction of $\mathrm{ABM}$ behaviour over a wide range of parameter combinations.

The term MM originates from the Design of Experiments literature (Wang and Shan, 2007; Montgomery, 2009). It was originally developed to study the effects of a set of explanatory variables on a response variable. Therein, optimization via response surface MMs was the most widely performed application (Barton, 1988). Both terms, surrogate models (Dey et al., 2017) or emulators (Conti and O'Hagan, 2010), can also be understood as MMs. Most commonly, they all treat a particular $\mathrm{ABM}$ as a white, grey or black box (Papadopoulos and Azar, 2016) and link the input and output values by aggregated functions (Barton, 1988; Friedman and Pressman, 1988; Friedman, 1996; Barton and Meckesheimer, 2006). As a result, MM significantly reduce simulation costs in terms of computational time and allow easier communication and understanding of simulation models' behavior (Kleijnen and Sargent, 2000; Mertens et al., 2018). This review will not consider other related concepts of MMs such as the model framework of concepts (Goldspink, 2000).

The aim of this review is to condense available information about common MM types used for various tasks related to $\mathrm{ABM}$ analysis and applications to guide modelers in choosing an appropriate

\footnotetext{
${ }^{1}$ We refer to both individual- and agent-based models synonymously as ABM.
} 
MM type for their research problem. For detailed information on specific MMs and their applications, it is advised to look for reviews or tutorials elsewhere like Barraquand and Murrell (2013), Barton (1988), Gore et al. (2017), Heard et al. (2015), Kalteh et al. (2008), Mertens et al. (2018), O’Hagan (2006), Oakley (2002) or Urban (2005). A methodology for rating MM quality and implementation effort in an ABM context was developed and applied for the reviewed publications by eight different raters with varying mathematical skills and scientific backgrounds. This was done to support readers in their selection and application of a metamodel in an ABM context.

\subsection{Methods}

\subsubsection{Searching procedure}

We conducted a literature survey in Open Access databases (see Tab. 5.1) on the 17th, 18th, 21st and 24th of January 2019 and considered only peer-reviewed papers. For each database used, we performed ten searches combining the terms agent-based model and individual-based model with each of the following keywords: Approximation, emulator, metamodel, meta-model and surrogate. We did not limit the time frame of the results but took only a maximum of 50 results per search into account, sorted by their relevance. Papers containing a single or combinations of keyword(s) in their title, abstract, or keywords section were selected for review.

Table 5.1. Complete list of all databases used for the review presented. The survey was conducted in January 2019 without limiting the years of publication.

\begin{tabular}{ll}
\hline \multicolumn{1}{c}{ Database } & \multicolumn{1}{c}{ Website } \\
\hline Academic Search Complete & ebscohost.com/academic/academic-search-complete \\
Web of Science Core Collection & apps.webofknowledge.com \\
Google Scholar & scholar.google.de \\
Scopus & elsevier. com/solutions/scopus \\
\hline
\end{tabular}

\subsubsection{Categorization of MMs and purpose of application}

In contrast to Papadopoulos and Azar (2016), we do not sub-classify MMs into white (reduced order), gray (both physical equations and stochastically estimated parameters) and black box (Machine Learning) surrogate models. Instead, we simply distinguish them according to their approach to describe the link between input and output variables as deterministic (e.g. Differential Equation) and stochastic 
(e.g. Machine Learning) MMs, respectively. We thus assign, for example, a Partial Differential Equation used for upscaling (e.g. Moorcroft et al., 2001) to the family of deterministic MMs, whereas Bayesian Emulators applied for calibration (e.g. Bijak et al., 2013) are considered as stochastic MMs.

The MMs were first subdivided into two main classes namely deterministic and stochastic models depending on whether they consider probability distributions linked to the input, output, or processes described by the ABM.

The classes were further subdivided into six model families that comprise different MM types (Tab. 5.2). In this sense, all MM family names resemble the so-called suitcase phrases and do not necessarily share all attributes or requirements of their namesake in a mathematical context. The names of the model types were directly extracted from the accepted papers without any adjustments. Appendix A provides complete information about the reviewed papers and the corresponding model families and types.

We categorized the purpose of each MM exclusively based on the declaration of the particular authors (Tab. 5.3). Notably, we understand parameter fitting as calibration incorporating calibration, parameterization or optimization in accordance to Railsback and Grimm (2012).

Table 5.2. MM classification derived from the accepted papers with MM applications in an ABM context. The differentiation between deterministic and stochastic models depend on whether probability distributions of input, output or processes described by the emulated ABM were taken into account. Model families represent the so-called suitcase phrases, which are not necessarily mathematical definitions for all MM types included in the family.

\begin{tabular}{lll}
\hline Model Class & \multicolumn{1}{c}{ Model Family } & \multicolumn{1}{c}{ Model Type } \\
\hline Deterministic & Ordinary Functional Equation & $\begin{array}{l}\text { Difference Equation, Equation-free Modeling, System Dynamics } \\
\text { Model }\end{array}$ \\
& Differential Equation & $\begin{array}{l}\text { Compartment ordinary Differential Equation (CODE), Ordinary Dif- } \\
\text { ferential Equation (ODE), Partial Differential Equation (PDE) }\end{array}$ \\
Stochastic & Regression & $\begin{array}{l}\text { First-order Regression, Linear Regression, Polynomial Regression, } \\
\text { Weighted ordinary Least Squares Regression }\end{array}$ \\
& Bayesian Emulator & $\begin{array}{l}\text { Approximate Bayesian Computation (ABC), Dynamic Linear Model } \\
\text { Gaussian process, Gaussian Process, Spatial correlation (Kriging), } \\
\text { Parametric Likelihood Approximation }\end{array}$ \\
& Machine Learning & $\begin{array}{l}\text { Decision Tree, Decision Tree Ensemble, Feature } \\
\text { Selection, Radial Basis Function Network, Random Forest, Support } \\
\text { Vector Regression, Symbolic } \\
\text { Regression }\end{array}$ \\
& \\
& Transition Matrices \\
\hline
\end{tabular}


Table 5.3. Common purposes of emulating ABMs by means of MMs.

\begin{tabular}{ll}
\hline \multicolumn{1}{c}{ Purpose } & \multicolumn{1}{c}{ Description } \\
\hline Calibration & $\begin{array}{l}\text { Find reasonable values for input parameters (Friedman and Pressman, 1988; Barton, 1988; } \\
\text { Friedman, 1996; Kleijnen and Sargent, 2000; Barton and Meckesheimer, 2006). }\end{array}$ \\
Prediction & $\begin{array}{l}\text { Predict model behavior for new scenarios or parameter values while replacing the simulation } \\
\text { model (Kleijnen and Sargent, 2000). Also known as exploratory analysis (Bigelow and Davis, }\end{array}$ \\
& $\begin{array}{l}\text { 2002), what-if analysis (Barton and Meckesheimer, 2006) or exploration/ inverse exploration } \\
\text { (Friedman and Pressman, 1988; Friedman, 1996). }\end{array}$ \\
Sensitivity analysis & $\begin{array}{l}\text { Explore model output sensitivity to changes in parameter values (Railsback and Grimm, 2012; } \\
\text { Thiele et al., 2014; Ligmann-Zielinska et al., 2020). }\end{array}$ \\
& $\begin{array}{l}\text { Scale the model to a coarser spatial resolution (Cipriotti et al., 2016) or from individuals to popu- } \\
\text { lations (Campillo and Champagnat, 2012). }\end{array}$ \\
\hline
\end{tabular}

\subsubsection{Assessment of MM quality and implementation effort}

In the following paragraphs, we briefly describe how we rated the MM's quality and implementation effort. For more in-depth information on the procedure as well as for some examples of each rating criterion, see Appendix C. This guide was used to rate each MM application and to calculate the mean quality and implementation effort.

An inter-rater reliability was calculated using the icc function of the R package irr version 0.84.1 (Gamer et al., 2019). Following Koo and Li (2016), we applied a two-way mixed effects model (all selected raters were the only one of interest), using average as type (we want to use the mean ratings for each MM application) and agreement as definition since we had sought to evaluate the agreement among the raters.

Table 5.4. Criteria applied for assessing the MM quality for the given purpose of emulating the ABM.

\begin{tabular}{lcccl}
\hline \multicolumn{1}{c}{ Criteria } & Low & $\begin{array}{c}\text { MM quality } \\
\text { Medium }\end{array}$ & High & Key Questions \\
\hline $\begin{array}{l}\text { Consideration of } \\
\text { Uncertainty (CU) }\end{array}$ & no & yes & with evaluation & $\begin{array}{l}\text { Did the authors give any assessment on the } \\
\text { uncertainties of the MM assumptions or re- } \\
\text { sults? }\end{array}$ \\
$\begin{array}{l}\text { Suitability Assess- } \\
\text { ment (SuA) }\end{array}$ & none or bad & $\begin{array}{c}\text { good (qualita- } \\
\text { tively) }\end{array}$ & $\begin{array}{c}\text { good (qualita- } \\
\text { tively) }\end{array}$ & $\begin{array}{l}\text { How did the authors state the suitability of the } \\
\text { MM for the given purpose? }\end{array}$ \\
$\begin{array}{l}\text { Number of Evalua- } \\
\text { tion Criteria (NE) }\end{array}$ & 1 & 2 & $>2$ & $\begin{array}{l}\text { How many different criteria were provided by } \\
\text { the authors for evaluating the MM suitability? }\end{array}$ \\
\hline
\end{tabular}

The quality of MM was assessed based on the assessment of the respective source authors using three different criteria (Tab. 5.4): Consideration of Uncertainty (CU), Suitability Assessment by 
source authors (SuA), and Number of Evaluation Criteria (NE). With the CU criterion, we evaluated how the authors considered uncertainties in the inputs and outputs of the respective MM family. In this criterion, the term no means that there was no explicit Consideration of Uncertainty given by the authors using the MM, while yes refers to those where they used at least some (quantitative) measures (e.g. error bars or $\mathrm{R}^{2}$ ). We assigned a high quality if the source authors had presented measures of uncertainty with a corresponding evaluation of such measures. The term suitability in SuA refers to the applicability of the given MM type (e.g., Approximate Bayesian Computation) to fulfill the particular purpose (e.g., calibration of an $\mathrm{ABM}$ ). A good MM evaluation by the authors was regarded as medium if the assessment is only based a qualitative statement (e.g., "the MM performed extremely well."). We adjudged suitability as good in those cases where the ABM emulation was quantitatively assessed with a positive result. The third criteria NE is self-explaining. For example, a basic linear regression model provides two criteria for evaluating suitability ( $\mathrm{R}$ squared for the goodness of fit and $\mathrm{p}$-value for evaluating the significance of the linear relationship between the input and output variables) and, thus, would receive a medium assessment for this specific criterion if the authors presented those criteria within their peer-reviewed research paper. Example statements like, the MM had a 61\% probability of selecting a parameter set that fitted all investigated outputs, or this procedure was successful in $92 \%$ of cases, revealing its great potential to assess parameters difficult to measure in nature, were considered as $\mathrm{SuA}=\operatorname{good}$ with $\mathrm{NE}=$ low.

The implementation effort of each MM family was assessed by the following three criteria (Tab. 5.5): Availability of Open Access Guiding source s (AG), RC overage (RC), and Out-of-the-Box Applicability (OA). Since we focus exclusively on the effort to implement MMs, computational cost has been absent in our consideration. The AG criterion evaluates the effort of finding help or further information for the potential MM application to own needs. If no sources could be found by performing a search in Google Scholar and Google.com using the MM type name as search query, the MM was regarded with a high implementation effort, while multiple usable sources (e.g. a page on Wikipedia.org and a mathematical blog entry) were considered as a medium implementation effort. Low efforts were assessed if there was one source giving a comprehensive tutorial on implementing the re- 
spective MM. The RC criterion focused on the free available statistical language $\mathrm{R}$ ( $\mathrm{R}$ Core Team, 2018). If one dedicated package is available to implement the whole MM, it was rated with a low implementation effort. If multiple R packages were necessary, a medium effort was given. We assigned a high implementation effort if the entire MM had to be developed from scratch. The last criterion OA assessed the possibility of MMs to be immediately usable (partly depends on the existing software). MMs were evaluated at a high implementation effort if the derivation of specific equations was required or some important assumptions had to be investigated for its use. Little adjustments correspond, for example, to the derivation of a linear model equation for the corresponding $\mathrm{R}$ function, while the application of an unsupervised artificial neural network was considered as a low implementation effort.

Table 5.5. Criteria applied for assessing the MM implementation effort for the given application aims.

\begin{tabular}{|c|c|c|c|c|}
\hline \multirow[t]{2}{*}{ Criteria } & \multicolumn{3}{|c|}{ Implementation effort } & \multirow[t]{2}{*}{ Key Questions } \\
\hline & Low & Medium & High & \\
\hline $\begin{array}{l}\text { Availability of Open } \\
\text { Access Guiding } \\
\text { sources (AG) }\end{array}$ & 1 good source & $\begin{array}{l}\text { multiple } \\
\text { sources }\end{array}$ & none & $\begin{array}{l}\text { Are there any openly accessible sources } \\
\text { like books or blogs that give an implemen- } \\
\text { tation guideline for the MM family of inter- } \\
\text { est? }\end{array}$ \\
\hline $\mathrm{R}$ coverage (RC) & 1 package & $\begin{array}{l}\text { multiple } \\
\text { packages }\end{array}$ & none & $\begin{array}{l}\text { Are there any dedicated } \mathrm{R} \text { packages to im- } \\
\text { plement the given MM? }\end{array}$ \\
\hline $\begin{array}{l}\text { Out-of-the-Box Ap- } \\
\text { plicability (OA) }\end{array}$ & $\begin{array}{c}\text { no } \\
\text { adjustments }\end{array}$ & $\begin{array}{l}\text { little } \\
\text { adjustments }\end{array}$ & $\begin{array}{c}\text { no } \\
\text { adjustments }\end{array}$ & $\begin{array}{l}\text { Is it necessary to develop an own equation } \\
\text { from scratch for the MM to be applicable? }\end{array}$ \\
\hline
\end{tabular}

Using the average value of all raters of each criterion, we conducted an overall assessment of quality and implementation effort of each MM application. Mean ratings were then analyzed separately for quality and implementation effort using the five-level classification (low, low-medium, medium, medium-high and high) displayed in Tab. 5.6. If, for example, a MM application received a high SuA, a high NE and a medium CU, a high overall MM quality was given. These overall assessments were used to generate a plot for each application aim (Tab. 5.3) depicting the MM quality in the dependency of the MM implementation effort. Within these plots, a bisecting line was drawn for visualizing the 1:1 ration of quality and implementation effort and highlight favorable MMs scoring above this line and less favorable MMs staying below this line. 
Table 5.6. The overall MM quality and implementation effort was calculated for each application according to the mean ratings of each of the three criteria for quality (CU, NE and $\mathrm{SuA}$ ) and effort (AG, OA and RC).

\begin{tabular}{cccc}
\hline & Amount of Scores in & Overall MM quality / implementation effort \\
Medium & Level & high \\
high & Low & medium-high \\
2 & 0 & 0 & medium-high \\
2 & 1 & 1 & medium \\
1 & 0 & 0 & medium \\
1 & 2 & 1 & low-medium \\
0 & 1 & 2 & low-medium \\
0 & 3 & 1 & low \\
0 & 0 & 2 & low \\
\hline
\end{tabular}

\subsection{Results and Discussion}

Following the previously described selection criteria (see Method section), 27 different peer-reviewed journal papers published from 2005 to 2019 (Fig. 5.1) were accepted for the review (see Appendix B). With this we could extract 40 different MM applications in an ABM context (see Appendix A).

\section{Sensitivity Analysis}

For Sensitivity Analyses, Bayesian Emulators and Regressions have the highest MM quality indicating accessible implementation efforts (Fig. 5.2). Half of the reviewed publications with focus on Machine Learning scored above the bisecting line indicating a broad MM usage, while the remaining applications were either on or below the bisecting line. 


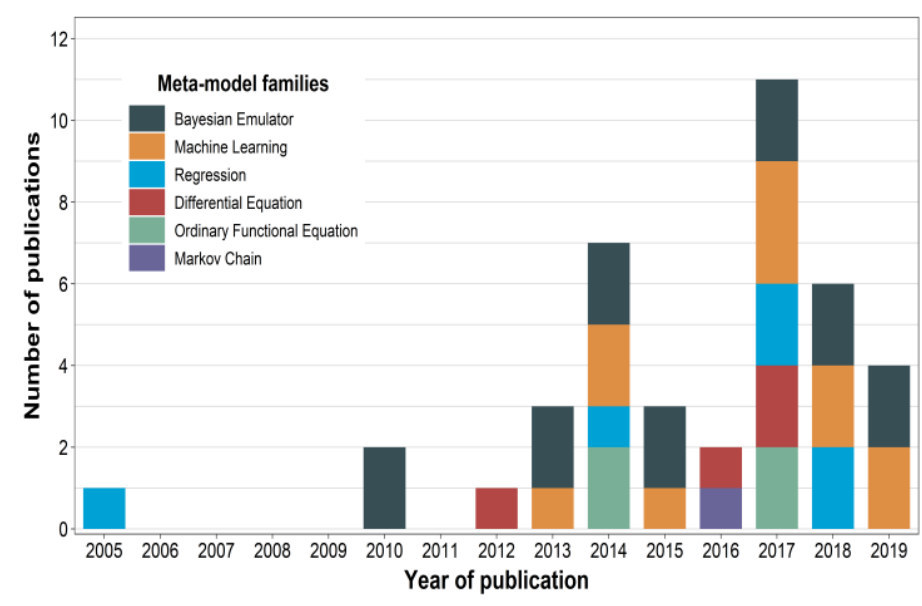

Figure 5.1. Number of reviewed papers and categorized MM families according to the publication year.

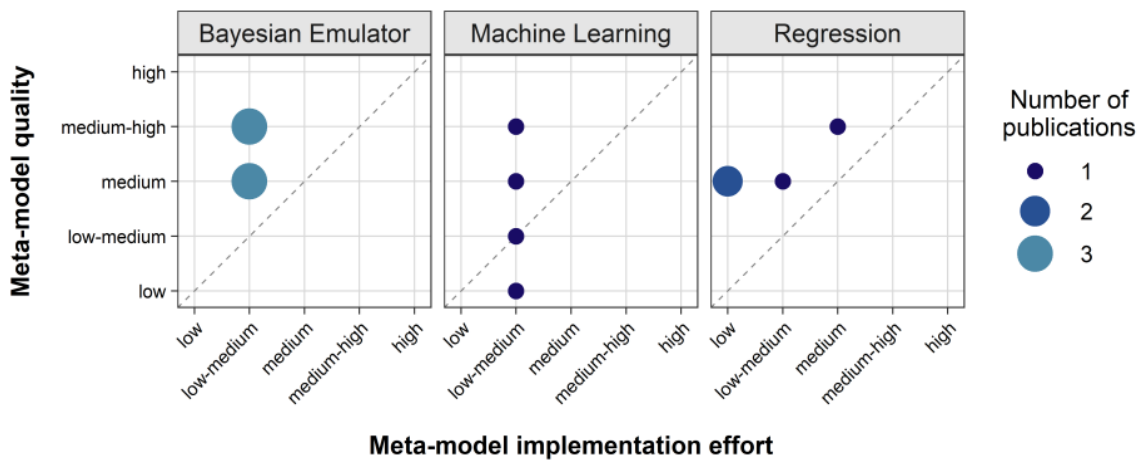

Figure 5.2. Results of the MM quality and implementation effort assessment for the application aim of sensitivity analysis.

Overall, we found the implementation effort for the three MM families (Bayesian Emulators, Machine Learning and Regression) to be reasonable due to a predominantly high RC (R Coverage) and the broad AG (availability of Open Access guiding sources) on these MMs. However, a shortcoming in the application of these three MM families for sensitivity analysis is their need for adjustments to be applicable for another ABM: there was not a single MM type within those MM families that could be reused without any changes. The superior qualities of Bayesian Emulators and Regression MMs result from the moderate to good SuA (Suitability Assessment by source authors) in addition to their moderate to good CU (Consideration of Uncertainty). The applied Machine Learning MMs for sensitivity analysis never exceeded a moderate NE (Number of Evaluation Criteria) while their CU and the SuA increased in the following order: Decision Tree Ensemble, Support Vector Regression, Symbolic Regression and Random Forest. 


\section{Calibration}

For calibration, Bayesian Emulators, Machine Learning and Regression MMs seem to be the preferable MM families since they constantly stay above the bisecting line (or thereon) indicating a beneficial MM quality to implementation effort ratio (Fig. 5.3). In contrast, Differential Equation and Ordinary Functional Equation MMs do not exceed or even reach the bisecting line and therefore seem to be less favorable MM families to be applied for the purpose of calibrating ABMs.

The overall low-medium implementation efforts of the three best scored MM families such as Bayesian Emulator, Machine Learning and Regression can be explained with their good to at least medium RC (RC overage) as well as the good to moderate AG (Availability of Guiding sources). Their OA (Out-of-the-Box Applicability) was never rated as low and always received medium or high assessments regarding their implementation efforts.

High implementation efforts of Differential Equations and Ordinary Functional Equations are due to considerably low OA because they have to be rebuilt entirely for every new ABM. Their AG and $\mathrm{RC}$ remain good to medium, emphasizing their broad usability. 


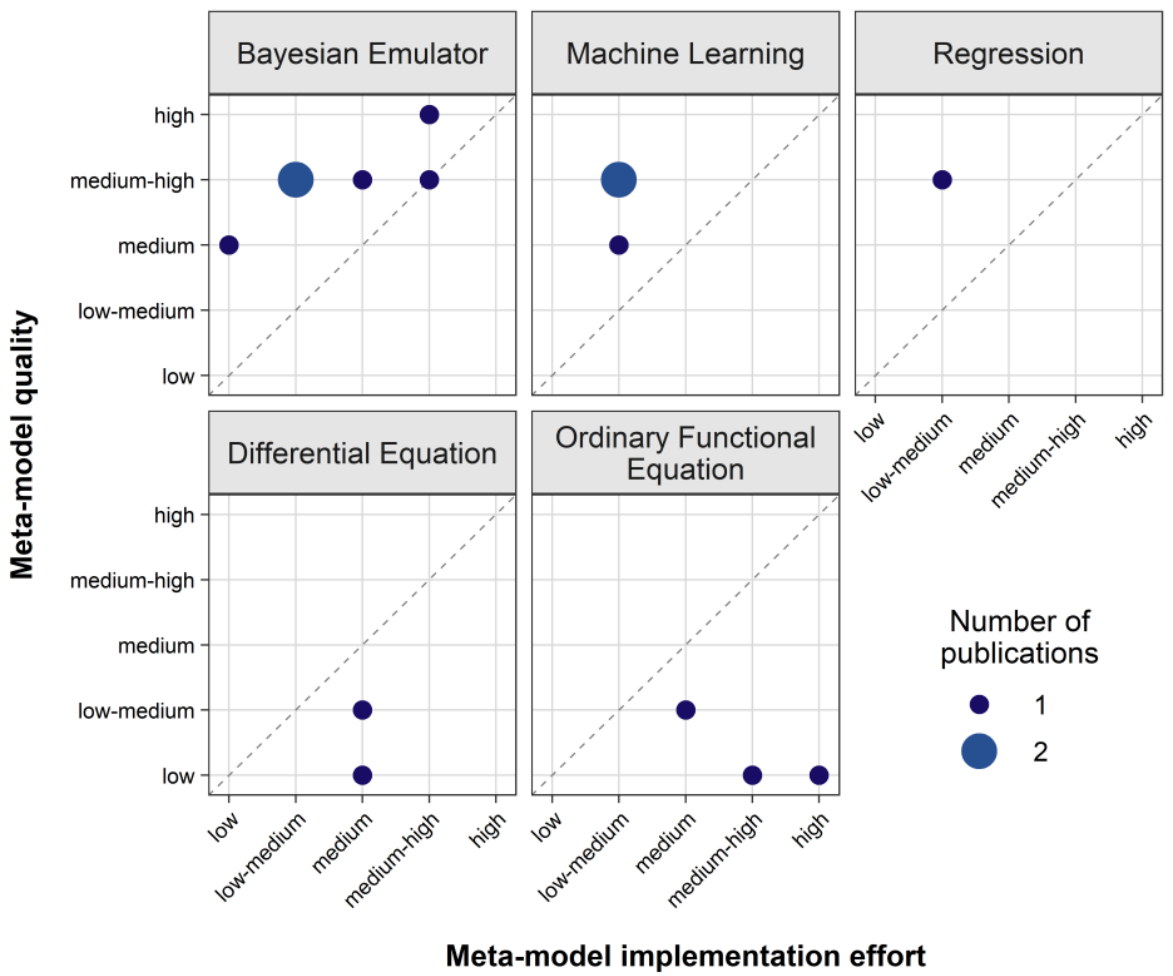

Figure 5.3. Results of the MM quality and implementation effort assessment for the application aim of calibration.

The superior MM qualities of Bayesian Emulators are due to their high NE as well as in-depth CU (Consideration of Uncertainty). Only SuA (Suitability Assessment of source authors) was poor to medium, indicating that not every MM type of this family suited the task of calibration as good as the others. Machine Learning MMs always achieved a good SuA while their CU and NE (Number of Evaluation Criteria) varied from medium to high.

The considerably poor qualities achieved by Differential Equations and Ordinary Functional Equations result from their low CU and NE. Nevertheless, the respective source authors assessed the suitability of these MMs qualitatively as good.

\section{Prediction}

In order to predict the behavior of ABMs, Bayesian Emulators and Machine Learning MMs seem to be the most favorable MM families since they continually exceed the bisecting line of 1:1 ratio for MM quality and implementation effort (Fig. 5.4). While the only Regression application for predicting ABMs achieves a low-medium MM quality as well as implementation effort signaling a trade-off be- 
tween prediction and implementation. Differential Equations as well as Ordinary Functional Equations consistently remain below the bisecting line.

For predicting ABMs behavior, Bayesian Emulators scored the best quality rating with varying implementation efforts. The low-medium effort of Gaussian Process Emulator originates from very good RC (R Coverage) as well as medium OA (Out-of-the-Box Applicability) and AG (Availability of Guiding sources). The medium-high effort of the dynamic linear model Gaussian Process is due to worse OA, AG as well as RC. The latter two criteria should be considered critically as we used the exact name presented here as a key phrase in our online research while looking for R packages and guiding sources. We could expect a lower implementation effort had we used a more flexible search term for this kind of MM type. The second best MM family for prediction of ABMs are Machine Learning models. Their considerably low implementation efforts are due to their broad RC and AG. OA varies around a medium ranking with decision trees achieving the highest rating. The varying quality within this MM family is because differentiating SuA (Suitability Assessment) by the respective source authors, while CU (Consideration of Uncertainty) is overall low and NE (Number of Eval-

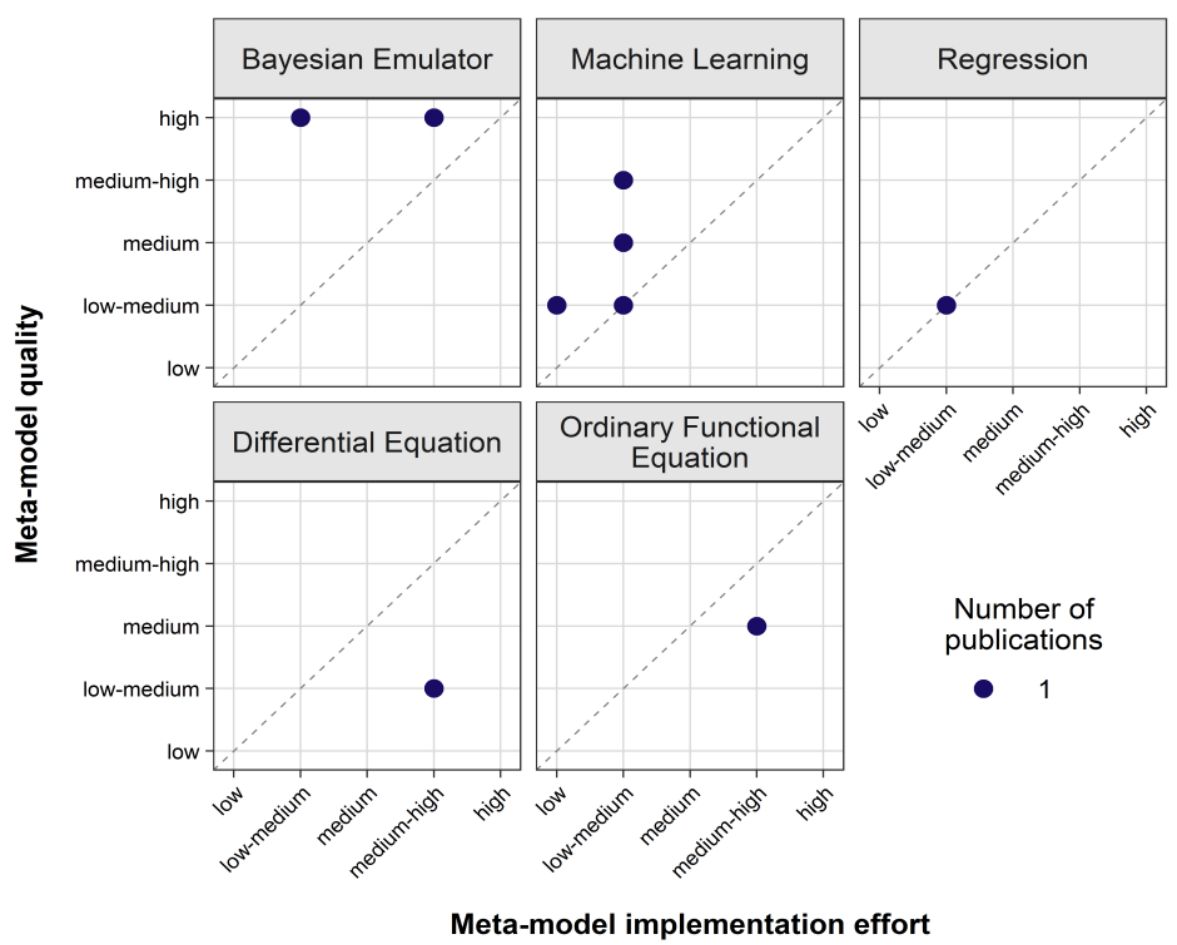

Figure 5.4. Results of the MM quality and implementation effort assessment for the application aim of prediction. 
uation Criteria) scores between low and medium. The highest quality is achieved by Random Forest for its comparable higher CU and NE. The Regression MM applied for predicting ABMs is a First Order Regression receiving lower quality ratings while still being good at SuA. The implementation effort consists of a medium OA (the formula of the linear model has to be adapted for every ABM) and a moderate RC, which could be caused by using the whole and exact model name for our online research of R packages.

The overall high implementation efforts of Differential Equations (Compartment Ordinary Differential Equation) and Ordinary Functional Equations (Systems Dynamic Model) while scoring only low-medium to medium qualities are due to their really low OA, since these MM families have to be rebuild anew entirely for each ABM applied. Furthermore, their CU as well as their NE is low, which together with only a qualitatively good SuA add up to medium qualities at best.

\section{Upscaling}

For upscaling ABMs only the Markov Chain MM exceeded a neutral MM quality and implementation effort ratio (Fig. 5.5). The Differential Equation MM stayed below the bisecting line, making it a less favorable choice of MM for upscaling ABMs.

The Markov Chain MM reached a medium quality because of the considerably high SuA (Suitability Assessment by source authors), low-medium CU (Consideration of Uncertainty) and NE (Number of Evaluation Criteria). The implementation effort is dominated by its poor OA (Out-of-theBox Applicability), meaning many adjustments are required to adapt this kind of MM to another ABM. The only accepted Differential Equation (Partial Differential Equation) scored a low OA since a new equation has to be derived for every application in ABMs.

\section{MM rating method and inter-rater reliability}

The inter-rater reliability never fell below a fair level and even achieved excellent evaluation for CU (Consideration of Uncertainty) and OA (Out-of-the-Box Applicability) (Tab. 5.7).

With eight raters and a sample size of $40 \mathrm{MM}$ applications, the requirements suggested by Koo and Li (2016) are met and exceeded, emphasizing the robustness of the inter-rater reliability re- 


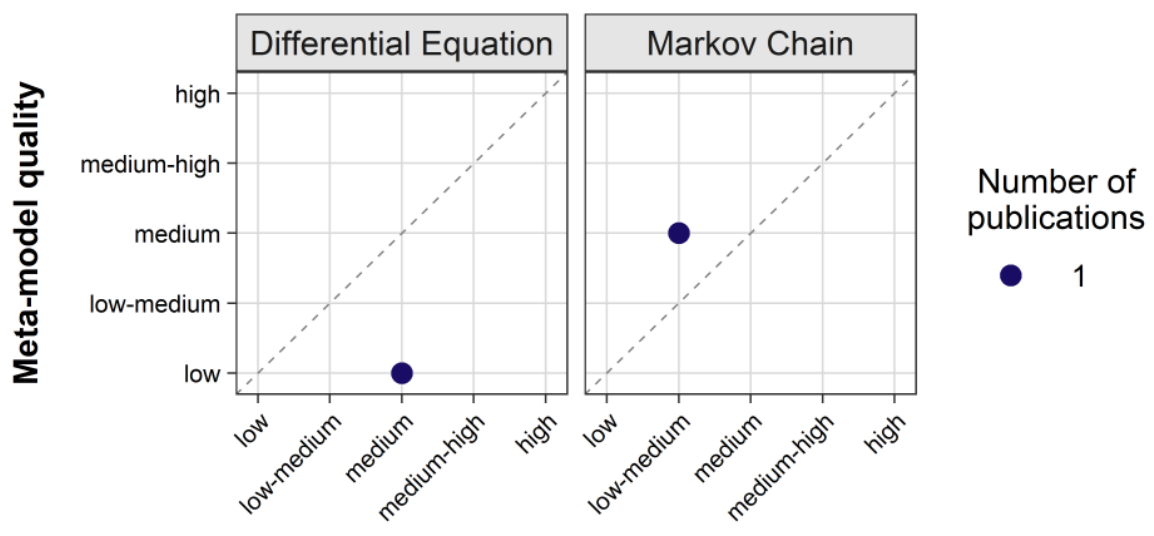

Meta-model implementation effort

Figure 5.5. Results of the MM quality and implementation effort assessment for the application aim of upscaling.

sults and therewith the results of the MM rating. Nevertheless, the calculated fair intra-class correlation coefficients for SuA (Suitability Assessment of source authors), AG (Availability of Guiding Literature) and RC (R Coverage) (Tab. 5.7) indicate a necessity to further improve the clarity of the rating instruction for these criteria.

One reason for the stronger variation inside the MM implementation effort criteria $\mathrm{AG}$ and $\mathrm{RC}$ lies within the diverse backgrounds of the raters, which participated in the MM assessment. Since the individual knowledge, the experiences with the corresponding MM types as well as the statistical software R were different (Appendix D), the assessment of a number of R packages needed to apply a given MM varied among reviewers. The only fair agreement within the MM quality criterion SuA could be because of the unclear instruction for cases in which the authors provided empirical proof for the suitability but never directly assessed it themselves qualitatively. In these cases, some raters gave a medium rating and others a high. Additional divergences emerged when the source authors did not provide any assessment but some raters were able to identify a good or bad fit by themselves while investigating the provided plots, highlighting disparities in certain instances. A more fine-grained analysis (e.g. five or seven scale evaluation) might reveal a clustering around high, medium and low with some within variations. 
Table 5.7. Calculated inter-rater reliability for the rating criteria with evaluation following Cicchetti (1994).

\begin{tabular}{llcc}
\hline \multicolumn{1}{c}{ Rating category } & \multicolumn{1}{c}{ Rating Criterion } & Inter-Rater Reliability \\
MM quality & Consideration of Uncertainty (CU) & 0.859 & $\begin{array}{c}\text { Evaluation } \\
\text { Excellent }\end{array}$ \\
& Suitability Assessment (SuA) & 0.556 & Fair \\
& Number of Evaluation Criteria (NE) & 0.721 & Good \\
$\begin{array}{l}\text { MM implementation } \\
\text { effort }\end{array}$ & $\begin{array}{l}\text { Availability of Open Access Guiding } \\
\text { sources (AG) }\end{array}$ & 0.461 & Fair \\
& R Coverage (RC) & 0.509 & Fair \\
& Out-of-the-Box Applicability (OA) & 0.773 & Excellent \\
\hline
\end{tabular}

\subsection{Conclusions}

Metamodelling is a promising approach to facilitate ABM calibration, sensitivity analysis, prediction and upscaling. We conducted a review that overviews the MM types used among their purposes. Within the 27 papers analysed, we identified 40 different $\mathrm{MM}$ applications. For each of them, we (PhD students and Postdocs with none up to moderate mathematical background) assessed the performance quality and the implementation effort. The methodology applied MM rating in this paper was validated by the fair to excellent intra-class correlation coefficients during the inter-rater reliability assessment. Our goal was to support MMs election for the various needs of daily ABM problems by highlighting the currently most promising MM types with an example each serving as a practical application guide:

\section{Sensitivity analysis:}

The easiest MMs to implement with a medium performance are Regression models (e.g. Polynomial Regression model). Several examples with stepwise guidance for implementation in R (Team, 2018) are provided by Thiele et al. (2014).

\section{Calibration:}

Approximate Bayesian Computation from the Bayesian Emulator family provides a good balance of effort and performance. Thiele et al. (2014) provides several basic implementation examples of ABM calibration with step-by-step guidance in R (Team, 2018). 


\section{Prediction:}

Gaussian Processes from the Bayesian Emulator MM family provide the best quality while offering low-medium implementation effort. In contrast, Random forest MMs (Machine Learning family) offer low-medium effort but only medium-high quality. An example on predicting new parameter combinations like an inverted calibration can be found in Peters et al. (2015).

\section{Upscaling:}

Transition Matrices from the Markov Chain MM family seem to be the most promising tool for scaling up ABMs. Note that we reviewed only two MMs on this application aim. The corresponding application can be found in Cipriotti et al. (2016).

This review was intended as a "first aid" for agent-based modelers who seek to improve the performance, optimization or analysis of their simulation model using a metamodel. Our motivation for this work ensued from our day-to-day modeling tasks. Please note that the review presented here can only provide an initial overview, which is primarily meant to stimulate and guide a potential reader through a self-exploration of the wide field of metamodels with ease. The examples presented here are not exhaustive and the field of metamodeling itself is constantly and rapidly developing. Particularly, the application of the potentials offered by various methods of artificial intelligence (with the branches of Machine Learning or deep learning) is just beginning to emerge. We would therefore like to motivate our readers to stay abreast on new developments in applying metamodeling approach to ABMs, and above all, try out metamodels in their own ways.

\section{Acknowledgements}

BP acknowledges funding from the PhD scholarship from the German Federal Environmental Foundation (Deutsche Bundesstiftung Umwelt-DBU). SF was supported by the German research Foundation (DFGprojectTI824/3-1). 


\section{General discussion and conclusions}

The overarching aim of my thesis was to study trait variability at population and community levels in arid environments and to assess the effect of the trait variability on the ability of plants to interact with neighbours and survive under harsh environmental conditions.

Trait-based modelling is a promising approach to study plant communities in temporally and spatially heterogeneous arid environments. Its advantages lie in the combination of the trait-based and ecological modelling approaches. Trait-based approaches allow to directly link plant characteristics to organismal functions (McGill et al., 2006; Violle et al., 2007). Ecological modelling approaches, in turn, provide opportunities to generalize observations, contribute to theory building (van der Putten et al., 2009; Herben and Wildová, 2012) and, in case of simulation models, investigate community dynamics under a wide range of environmental settings without endangering natural communities (Meyer et al., 2009; Zurell et al., 2010). Thus, to tackle the overarching aim of my thesis, I applied modelling approaches supported by literature reviews, field observations and empirical measurements of plant functional traits.

In the following, I briefly summarize key findings and contributions of each thesis chapter and show how they complement each other (Fig. 6). After this, I address the implications of these results for future theory building and practical applications for arid plant communities. 


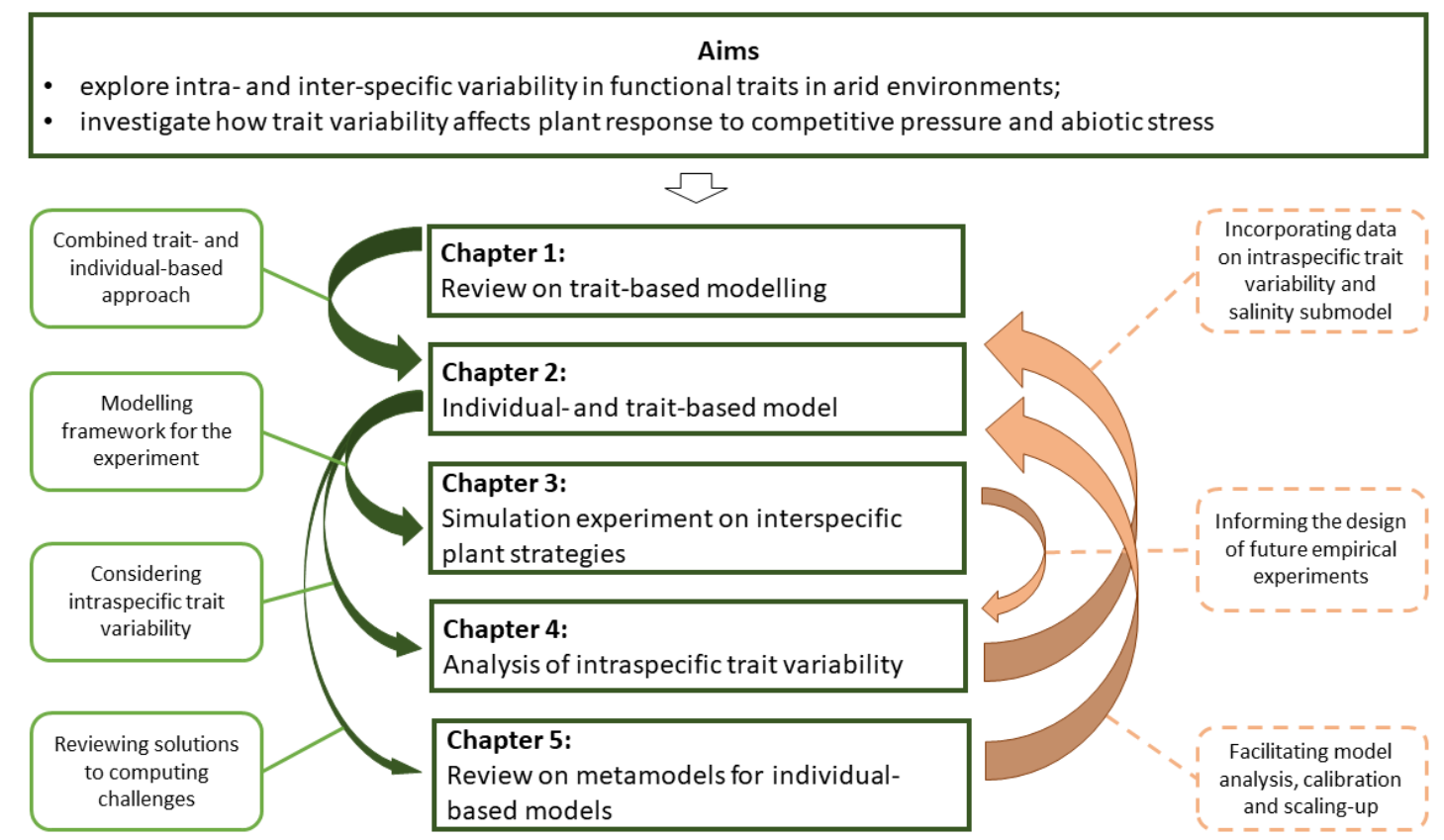

Figure 6. A conceptual scheme showing the flow of theoretical concepts and data from one chapter to another and links between all of them in order to achieve the overarching aim of the thesis. The rounded rectangle boxes with solid green borders on the left reflect the motivation for the next chapter and realized contribution, while the rounded rectangle boxes with dashed orange borders on the right show possible future contributions from one chapter to another.

In chapter 1 of this thesis, together with my co-authors, I reviewed the raise of trait-based modelling approaches in ecology. Trait-based models appear to be a powerful tool to tackle as diverse research questions as understanding mechanisms that drive plant growth, describing population and community development in space and time and explaining biodiversity. This application of trait-based models across different ecological disciplines can be explained by their intrinsic ability to facilitate parameterization, to scale up the embedded processes and to generalize the results. Hence, trait-based approaches have been successfully incorporated in conceptual models, statistical models, equationbased models and individual-based models. Individual-based models (Grimm and Railsback, 2005; DeAngelis and Mooij, 2005) are highly suitable for a combination with trait-based approaches. The reason for this is that trait values are assigned to an individual, and, thus, trait variation at the individual level can be considered (May et al., 2009; Scheiter et al., 2013; Weiss et al., 2014; Pontarp and Wiens, 2017). Moreover, spatially explicit individual-based models facilitate a direct link between traits and environmental factors and provide a suitable tool to study responses in community dynamics to environmental changes (McGill et al., 2006; Webb et al., 2010). However, such a detailed representation in these models comes at the cost of higher demands for parameterization and computing power 
(but see chapter 5). Ultimately, combined individual- and trait-based models were shown to be particularly promising when the feedback loop between the environment and a community should be taken into consideration in studies of community dynamics.

Putting our theoretical conclusion in chapter 1 on advantages of combined trait- and individual-based models into practice, together with my co-authors, I developed, implemented, documented and analysed the spatially explicit individual- and trait-based ATID-model (chapter 2). The novelty of this model is that it considers community dynamics of desert annuals from a plant traits point of view. In general, there are not many models known that describe desert vegetation in connection to their environment (James et al., 2005; Reick et al., 2013), but to my knowledge desert annuals are not in the scope of any of them. The sensitivity analysis of the model output demonstrated that the key drivers of community dynamics were more often plant functional traits than environmental factors. This finding might imply that desert annuals acquired fine-tuned trait-based strategies to tolerate abiotic stress, but not avoid it. Simultaneously, these strategies may indicate a high adaptability to an unpredictable environment (Adondakis and Venable, 2004; Venable et al., 2008). Among the plant functional traits involved in plant-plant interactions, the most influential traits were relative growth rate and maximum biomass. Among the traits involved in tolerating abiotic stress, the most influential traits were the amount of time in dormancy and probability to germinate. Soil water availability and precipitation were identified as the most influential environmental factors in line with empirical studies (Hegazy and Kabiel, 2007). Thus, focusing on traits to explore plant community dynamics in deserts not only implements the functional approach but also addresses key drivers of these dynamics.

The ATID-model from chapter 2 was applied for a simulation study on survival and performance of two annual species representing different survival strategies in chapter 3 . The model analysis in chapter 2 highlighted the importance of time in dormancy and germination probability but showed little influence of dispersal strategies for community dynamics. Therefore, to look at these processes in more details, together with my co-authors, I considered complex plant survival strategies integrating dispersal, dormancy, germination as well as later life-cycle stages. These strategies, labelled by us as a 'protective-competition' strategy and 'escape-colonization' strategy, synthesized 
three different concepts: seed strategies of escape in time and space (Venable and Lawlor, 1980), competition-colonization trade-off (Levins and Culver, 1971; Tilman, 1994) and a strategy classification suggested by Gutterman (1994). The resulting two-phase community dynamics agreed with the predictions of the competition-colonization trade-off, whereas the temporal dominance of one or the other strategy under different levels of temporal and spatial heterogeneity agreed with expectations for escape in time and space. This simulation experiment particularly demonstrates the potential of the ATID-model for studying the response of annual plant communities to environmental change. The new integrated plant strategy definition suggested in chapter 3, firstly, provided a more realistic representation of desert annual plant species and, secondly, granted a plausible explanation of observed patterns of community dynamics in line with the predictions of an established ecological theory, the competition-colonization trade-off.

To deepen the insights of the influence of environmental factors on community dynamics obtained in chapter 2 and chapter 3, together with my co-authors, I explored the response of average trait values and intraspecific trait spaces to imposed salinity stress in chapter 4 . Analysis of the data obtained from a nethouse experiment took into account the aridity, soil salinity and species richness of the origin of the plants in the experiment. Response to salinity stress was shaped to some extent by the origin of plants, for example, plants originating from highly arid sites did not respond by a substantial increase in stress-related intraspecific trait spaces. Additionally, salinity stress had a significant effect on the average values of functional traits. The results of this chapter, firstly, emphasized the strong influence of salinity stress on plant performance and, secondly, provided new insights on the shape and overlaps of intraspecific trait spaces of desert annuals. These insights should, thus, be reflected in future extensions of the ATID-model (chapter 2).

To address possible limitations of combined trait- and individual-based models in computing power (chapter 1) and to support future extensions and analysis of the ATID-model (chapter 2 and 3), we reviewed available metamodels for individual-based models in chapter 5. For purposes of sensitivity analysis, regression models were shown to be an appropriate choice, while for calibration, as it was implemented in chapter 3, Approximate Bayesian Computation performed better than other met- 
amodel types. According to the review rating, Gaussian Processes from the Bayesian emulator metamodel family are a promising approach if individual-based models aim at making predictions, whereas for scaling-up, transition matrices from the Markov Chain family are recommended. Applying these insights to the ATID-model (chapter 2), I advise to test replacing the model by Bayesian Emulators to reduce the complexity of the detailed model versions or implementing transition matrices to transfer the model to a coarser spatial resolution.

This thesis covers a number of facets of trait-based modelling approaches for arid environments and tackles some challenges and limitations associated with them, but this work also paves the way for future research in this direction. In chapter 1 , I advocate for applying combined trait- and individual-based modelling approaches in order to study and to understand the mechanisms driving desert plant communities. Such an approach also provides a functional link between traits of individuals, their environment and emerging community properties. In this way, we can draw meaningful conclusions on how different traits influence the ability of plants to tolerate environmental stress and affect plant-plant interactions. The implementation of a combined trait- and individual-based approach in the development of the ATID-model in chapter 2 highlights the greater importance of functional traits as drivers of desert annual plant communities than environmental factors. This finding implies high adaptability of desert plants to environmental unpredictability. The simulation experiments in chapter 3 demonstrated that considering more realistic and integrative plant strategies such as 'protectivecompetition' and 'escape-colonization' strategies provide good explanations of observed patterns in community dynamics. These results also supported the idea that interspecific trait variability may express species adaptations to spatial and temporal heterogeneity of the environment. However, the individual response might differ as the analyses of intraspecific variability showed in chapter 4 and may even play a more important role than interspecific variability in adaptation to highly heterogeneous environments (Read et al., 2014; Jung et al., 2014). The significant influences of salinity stress on functional plant traits and observations on changes in trait spaces derived from the analysis in chapter 4 are to be incorporated in the next versions of the ATID-model (chapter 2). 
The flexible framework of the model allows for further extensions in form of additional submodels, e.g. to model salinity effects (based on chapter 4), or by assigning trait distributions instead of constant values to individuals to capture the effects of intraspecific trait variability. Intraspecific trait variability is already included to some extent in the ATID-model, but only as emerging variability such as in the case of individual biomass. I expect that explicitly considering intraspecific trait variability as input in the model might provide longer coexistence of species in the simulated communities described in chapter 3. This effect is expected to be stronger under highly heterogeneous environments, as the individual response is a source of population plasticity. Additionally, including intraspecific trait variability in the ATID-model would allow for exploring the effects of environmental filtering on trait spaces in the model simulations to enrich the empirical results on trait spaces from chapter 4. To overcome possible computing challenges due to the more detailed representation of communities I recommend to use metamodels, e.g. for predicting model behaviour, as reviewed and assessed in chapter 5 .

In general, incorporating more realistic functional trait data and environmental data will improve model performance and its predictive power in case of applying the model with a prediction purpose. This step asks for joint empirical-modelling efforts as model outcomes should be compared to reality for validation and mutual improvement of models and experiments. In this regard, the plea from chapter 3 to model plant strategies as closely as possible to real species and to include more species in model communities will facilitate matching the simulated world with reality. Moreover, joint empirical-modelling approaches contribute not only to model parameterization, but also to the experimental design of empirical studies. Starting research by investigating potential hypotheses with correspondingly tailored versions of the ATID-model would help selecting the measurements to be taken and possible scenarios to be explored in the experiment as well as factors to pay attention to throughout the field observations. For example, it would be interesting to use the results obtained in the simulation experiment in chapter 3 as hypotheses for field observations. Such a field observation would require precise precipitation and topographical data, measurement of plant biomass in the field and 
recording of species abundance over several years to assess the relative dominance of different survival strategies on the short and long term.

To conclude, my thesis, in line with the main aims of the umbrella project "Population- and community- level plant trait variability in changing arid environments", explored the role of trait variability at the population level (analysis of the nethouse experiment) and community level (simulation experiment) in shaping the response to aridity and salinity. I identified a set of plant functional traits that are most influential for community dynamics (model analysis). Additionally, I showed that two groups of traits distinguished as those involved in withstanding harsh environmental conditions and those involved in plant-plant interactions are equally driving community dynamics (model analysis and simulation experiment). Moreover, I demonstrated the suitability of a combined trait-based and individual-based approach for studying community dynamics of desert annual plants and underlined the importance of the cooperation between modelling and empirical research, which contributes not only to model parameterization but also to fine-tuned experimental designs of empirical studies. Finally, with this thesis, I provided a modelling framework for further research on the mechanisms underlying community dynamics and understanding the fate of desert communities in the face of global changes. 


\section{References}

Abrego, N., Norberg, A., Ovaskainen, O., 2017. Measuring and predicting the influence of traits on the assembly processes of wood-inhabiting fungi. J. Ecol. 105, 1070-1081. https://doi.org/10.1111/1365-2745.12722

Acevedo-Trejos, E., Brandt, G., Bruggeman, J., Merico, A., 2015. Mechanisms shaping size structure and functional diversity of phytoplankton communities in the ocean. Sci. Rep. 5, 17-20. https://doi.org/10.1038/srep08918

Acevedo-Trejos, E., Marañón, E., Merico, A., 2018. Phytoplankton size diversity and ecosystem function relationships across oceanic regions. Proc. R. Soc. B Biol. Sci. 285. https://doi.org/10.1098/rspb.2018.0621

Adler, P.B., Salguero-Gomez, R., Compagnoni, A., Hsu, J.S., Ray-Mukherjee, J., Mbeau-Ache, C., Franco, M., 2014. Functional traits explain variation in plant life history strategies. Proc. Natl. Acad. Sci. 111, 740-745.

https://doi.org/10.1073/pnas.1315179111

Adondakis, S., Venable, D.L., 2004. Dormancy and germination in a guild of sonoran desert annuals. Ecology 85, 25822590. https://doi.org/10.1890/03-0587

Ahmed, I.M., Dai, H., Zheng, W., Cao, F., Zhang, G., Sun, D., Wu, F., 2013. Genotypic differences in physiological characteristics in the tolerance to drought and salinity combined stress between Tibetan wild and cultivated barley. Plant Physiol. Biochem. 63, 49-60. https://doi.org/10.1016/j.plaphy.2012.11.004

Albert, C.H., Thuiller, W., Yoccoz, N.G., Douzet, R., Aubert, S., Lavorel, S., 2010. A multi-trait approach reveals the structure and the relative importance of intra- vs. interspecific variability in plant traits: Intra- vs. interspecific variability in plant traits. Funct. Ecol. 24, 1192-1201. https://doi.org/10.1111/j.1365-2435.2010.01727.x

Albert, C.H., Thuiller, W., Yoccoz, N.G., Soudant, A., Boucher, F., Saccone, P., Lavorel, S., 2017. Intraspecific functional variability: extent, structure and sources of variation. J. Ecol. 604-613. https://doi.org/10.1111/j.13652745.2010.01651.x@10.1111/(ISSN)1365-2745.1017

Albert, C.H., Grassein, F., Schurr, F.M., Vieilledent, G., Violle, C., 2011. When and how should intraspecific variability be considered in trait-based plant ecology? Perspect. Plant Ecol. Evol. Syst. 13, 217-225. https://doi.org/10.1016/J.PPEES.2011.04.003

Alexandridis, N., Dambacher, J.M., Jean, F., Desroy, N., Bacher, C., 2017. Qualitative modelling of functional relationships in marine benthic communities. Ecol. Modell. 360, 300-312. https://doi.org/10.1016/j.ecolmodel.2017.07.021

Ali, A.A., Medlyn, B.E., Aubier, T.G., Crous, K.Y., Reich, P.B., 2015. Elevated carbon dioxide is predicted to promote coexistence among competing species in a trait-based model. Ecol. Evol. 5, 4717-4733. https://doi.org/10.1002/ece3.1733

Ali, A.A., Medlyn, B.E., Crous, K.Y., Reich, P.B., 2013. A trait-based ecosystem model suggests that long-term responsiveness to rising atmospheric $\mathrm{CO} 2$ concentration is greater in slow-growing than fast-growing plants. Funct. Ecol. 27, 10111022. https://doi.org/10.1111/1365-2435.12102

Allison, S.D., 2012. A trait-based approach for modelling microbial litter decomposition. Ecol. Lett. 15, 1058-1070. https://doi.org/10.1111/j.1461-0248.2012.01807.x

Allison, S.D., Goulden, M.L., 2017. Consequences of drought tolerance traits for microbial decomposition in the DEMENT model. Soil Biol. Biochem. 107, 104-113. https://doi.org/10.1016/j.soilbio.2017.01.001

Andersen, K.H., Beyer, J.E., 2006. Asymptotic Size Determines Species Abundance in the Marine Size Spectrum. Am. Nat. 168, 54-61. https://doi.org/10.1086/504849

Andersen, K.H., Brander, K., Ravn-Jonsen, L., 2015. Trade-offs between objectives for ecosystem management of fisheries. Ecol. Appl. 25, 1390-1396. https://doi.org/10.1890/14-1209.1

Andersen, K.H., Pedersen, M., 2010. Damped trophic cascades driven by fishing in model marine ecosystems. Proc. R. Soc. B Biol. Sci. 277, 795-802. https://doi.org/10.1098/rspb.2009.1512

Andersen, K.H., Rice, J.C., 2010. Direct and indirect community effects of rebuilding plans. ICES J. Mar. Sci. 67, 19801988. https://doi.org/10.1093/icesjms/fsq035

Aronson, J., Kigel, J., Shmida, A., 1993. Reproductive allocation strategies in desert and Mediterranean populations of annual plants grown with and without water stress. Oecologia 93, 336-342. https://doi.org/10.1007/BF00317875

Astegiano, J., Guimarães, P.R., Cheptou, P.O., Vidal, M.M., Mandai, C.Y., Ashworth, L., Massol, F., 2015. Persistence of plants and pollinators in the face of habitat loss: Insights from trait-based metacommunity models, 1st ed, Advances in Ecological Research. Elsevier Ltd. https://doi.org/10.1016/bs.aecr.2015.09.005 
Aubry-Kientz, M., Rossi, V., Boreux, J.J., Hérault, B., 2015. A joint individual-based model coupling growth and mortality reveals that tree vigor is a key component of tropical forest dynamics. Ecol. Evol. 5, 2457-2465. https://doi.org/10.1002/ece3.1532

Auger, S., Shipley, B., 2013. Inter-specific and intra-specific trait variation along short environmental gradients in an oldgrowth temperate forest. J. Veg. Sci. 24, 419-428. https://doi.org/10.1111/j.1654-1103.2012.01473.x

Barraquand, F., Murrell, D. J. (2013). Scaling up predator-prey dynamics using spatial moment equations. Methods in Ecology and Evolution, 4 (3), 276-289

Barton, R. R.,1988. Metamodeling: A state of the art review. In A.Thesen, H. Grant \& K. D. Kelton (Eds.), 1987. Winter Simulation Conference Proceedings, 237-244. San Diego, CA: IEEE

Barton, R. R., Meckesheimer, M., 2006. Chapter18 Metamodel-Based Simulation Optimization. In Handbooks in Operations research and Management Science, 13, 535-574.

Beckett, S.J., Weitz, J.S., 2018. The Effect of Strain Level Diversity on Robust Inference of Virus-Induced Mortality of Phytoplankton. Front. Microbiol. 9, 1-15. https://doi.org/10.3389/fmicb.2018.01850

Bennett, M.G., Whiles, M.R., Whitledge, G.W., 2016. Population-level responses of life history traits to flow regime in three common stream fish species. Ecohydrology 9, 1388-1399. https://doi.org/10.1002/eco.1734

Berge, T., Chakraborty, S., Hansen, P.J., Andersen, K.H., 2017. Modeling succession of key resource-harvesting traits of mixotrophic plankton. ISME J. 11, 212-223. https://doi.org/10.1038/ismej.2016.92

Berger, U., Piou, C., Schiffers, K., Grimm, V., 2008. Competition among plants: Concepts, individual-based modelling approaches, and a proposal for a future research strategy. Perspect. Plant Ecol. Evol. Syst. 9, 121-135. https://doi.org/10.1016/j.ppees.2007.11.002

Berkowicz, S.M., Blume, H.-P., Yair, A., 1995. The Arid Ecosystems Research Centre of the Hebrew University of Jerusalem. Arid Ecosyst. Res. Cent. Hebr. Univ. Jerus. 1-11.

Bestion, E., García-Carreras, B., Schaum, C.E., Pawar, S., Yvon-Durocher, G., 2018. Metabolic traits predict the effects of warming on phytoplankton competition. Ecol. Lett. 21, 655-664. https://doi.org/10.1111/ele.12932

Bhaskar, R., Dawson, T.E., Balvanera, P., 2014. Community assembly and functional diversity along succession postmanagement. Funct. Ecol. 28, 1256-1265. https://doi.org/10.1111/1365-2435.12257

Bigelow, J. H., Davis, P. K., 2002. Developing improved metamodels by combining phenomenological reasoning with statistical methods. In A. F. Sisti \& D. A. Trevisani (Eds.), Enabling Technologies for Simulation Science VI, July2002, 167-180. Bellingham, WAq: SPIE- the International Society for Optical Engineering

Bijak, J., Hilton, J., Silverman, E. \& Cao, V. D., 2013. Reforging the wedding ring: Exploring a semi-artificial model of population for the United Kingdom with Gaussian process emulators. Demographic research, 29 (December2013), 729-766

Biswas, S.R., Mallik, A.U., Braithwaite, N.T., Wagner, H.H., 2016. A conceptual framework for the spatial analysis of functional trait diversity. Oikos 125, 192-200. https://doi.org/10.1111/oik.02277

Bittebiere, A., Saiz, H., Mony, C., 2019. New insights from multidimensional trait space responses to competition in two clonal plant species. Funct. Ecol. 33, 297-307. https://doi.org/10.1111/1365-2435.13220

Blonder, B., Harris, D.J., 2018. hypervolume: High Dimensional Geometry and Set Operations Using Kernel Density Estimation, Support Vector Machines, and Convex Hulls. R package version 2.0.11. https://CRAN.Rproject.org/package=hypervolume

Blonder, B., Lamanna, C., Violle, C., Enquist, B.J., 2014. The n -dimensional hypervolume: The n-dimensional hypervolume. Glob. Ecol. Biogeogr. 23, 595-609. https://doi.org/10.1111/geb.12146

Bolker, B.M., Pacala, S.W., 1999. Spatial Moment Equations for Plant Competition: Understanding Spatial Strategies and the Advantages of Short Dispersal. Am. Nat. 153, 575-602. https://doi.org/10.1086/303199

Bolnick, D.I., Amarasekare, P., Araújo, M.S., Bürger, R., Levine, J.M., Novak, M., Rudolf, V.H.W., Schreiber, S.J., Urban, M.C., Vasseur, D.A., 2011. Why intraspecific trait variation matters in community ecology. Trends Ecol. Evol. 26, $183-192$. https://doi.org/10.1016/j.tree.2011.01.009

Bonachela, J.A., Klausmeier, C.A., Edwards, K.F., Litchman, E., Levin, S.A., 2016. The role of phytoplankton diversity in the emergent oceanic stoichiometry. J. Plankton Res. 38, 1021-1035. https://doi.org/10.1093/plankt/fbv087

Bonser, S.P., 2006. Form Defining Function: Interpreting Leaf Functional Variability in Integrated Plant Phenotypes. Oikos $114,187-190$. 
Bouskill, N.J., Tang, J., Riley, W.J., Brodie, E.L., 2012. Trait-based representation of biological nitrification: Model development, testing, and predicted community composition. Front. Microbiol. 3, 1-17. https://doi.org/10.3389/fmicb.2012.00364

Bown, J.L., Pachepsky, E., Eberst, A., Bausenwein, U., Millard, P., Squire, G.R., Crawford, J.W., 2007. Consequences of intraspecific variation for the structure and function of ecological communities. Part 1. Model development and predicted patterns of diversity. Ecol. Modell. 207, 264-276. https://doi.org/10.1016/j.ecolmodel.2007.05.004

Brochier, T., Ecoutin, J.M., de Morais, L.T., Kaplan, D.M., Lae, R., 2013. A multi-agent ecosystem model for studying changes in a tropical estuarine fish assemblage within a marine protected area. Aquat. Living Resour. 26, 147-158. https://doi.org/10.1051/alr/2012028

Brousseau, P.-M., Gravel, D., Handa, I.T., 2018. On the development of a predictive functional trait approach for studying terrestrial arthropods. J. Anim. Ecol. 1209-1220. https://doi.org/10.1111/1365-2656.12834

Bruggeman, J., 2009. Succession in plankton communities - A trait-based perspective (PhD thesis). Dep. Theor. Biol., Vrije Universiteit Amsterdam, the Netherlands.

Bruggeman, J., Kooijman, S.A.L.M., 2007. A biodiversity-inspired approach to aquatic ecosystem modeling. Limnol. Oceanogr. 52, 1533-1544. https://doi.org/10.4319/lo.2007.52.4.1533

Buchmann, C.M., Schurr, F.M., Nathan, R., Jeltsch, F., 2011. An allometric model of home range formation explains the structuring of animal communities exploiting heterogeneous resources. Oikos 120, 106-118. https://doi.org/10.1111/j.16000706.2010.18556.x

Burton, J.I., Perakis, S.S., McKenzie, S.C., Lawrence, C.E., Puettmann, K.J., 2017. Intraspecific variability and reaction norms of forest understorey plant species traits. Funct. Ecol. 31, 1881-1893. https://doi.org/10.1111/1365-2435.12898

Cadotte, M.W., 2007. Competition-Colonization Trade-Offs and Disturbance Effects at Multiple Scales. Ecology 88, 823829. https://doi.org/10.1890/06-1117

Calcagno, V., Mouquet, N., Jarne, P., David, P., 2006. Coexistence in a metacommunity: the competition-colonization tradeoff is not dead. Ecol. Lett. 9, 897-907. https://doi.org/10.1111/j.1461-0248.2006.00930.x

Campillo, F., Champagnat, N. 2012. Simulation and analysis of an individual-based model for graph-structured plant dynamics. Ecological Modelling, 234, 93-105

Carmona, C.P., de Bello, F., Mason, N.W.H., Lepš, J., 2016. Traits Without Borders: Integrating Functional Diversity Across Scales. Trends Ecol. Evol. 31, 382-394. https://doi.org/10.1016/j.tree.2016.02.003

Castellani, M., Rosland, R., Urtizberea, A., Fiksen, Ø., 2013. A mass-balanced pelagic ecosystem model with size-structured behaviourally adaptive zooplankton and fish. Ecol. Modell. 251, 54-63. https://doi.org/10.1016/j.ecolmodel.2012.12.007

Castro, B.M., Moriuchi, K.S., Friesen, M.L., Badri, M., Nuzhdin, S.V., Strauss, S.Y., Cook, D.R., von Wettberg, E., 2013. Parental environments and interactions with conspecifics alter salinity tolerance of offspring in the annual Medicago truncatula. J. Ecol. 101, 1281-1287. https://doi.org/10.1111/1365-2745.12125

Chakraborty, S., Nielsen, L.T., Andersen, K.H., 2017. Trophic Strategies of Unicellular Plankton. Am. Nat. 189, E77-E90. https://doi.org/10.1086/690764

Chamberlain, S.A., Bronstein, J.L., Rudgers, J.A., 2014. How context dependent are species interactions? Ecol. Lett. 17, 881-890. https://doi.org/10.1111/ele.12279

Chapin, F.S., Zavaleta, E.S., Eviner, V.T., Naylor, R.L., Vitousek, P.M., Reynolds, H.L., Hooper, D.U., Lavorel, S., Sala, O.E., Hobbie, S.E., Mack, M.C., Díaz, S., 2000. Consequences of changing biodiversity. Nature 405, 234-242. https://doi.org/10.1038/35012241

Chauvet, M., Kunstler, G., Roy, J., Morin, X., 2017. Using a forest dynamics model to link community assembly processes and traits structure. Funct. Ecol. 31, 1452-1461. https://doi.org/10.1111/1365-2435.12847

Chavana-Bryant, C., Malhi, Y., Wu, J., Asner, G.P., Anastasiou, A., Enquist, B.J., Cosio Caravasi, E.G., Doughty, C.E., Saleska, S.R., Martin, R.E., Gerard, F.F., 2017. Leaf aging of Amazonian canopy trees as revealed by spectral and physiochemical measurements. New Phytol. 214, 1049-1063. https://doi.org/10.1111/nph.13853

Chave, J., Andalo, C., Brown, S., Cairns, M.A., Chambers, J.Q., Eamus, D., Fölster, H., Fromard, F., Higuchi, N., Kira, T., Lescure, J.P., Nelson, B.W., Ogawa, H., Puig, H., Riéra, B., Yamakura, T., 2005. Tree allometry and improved estimation of carbon stocks and balance in tropical forests. Oecologia 145, 87-99. https://doi.org/10.1007/s00442-005-0100-x

Chave, J., Coomes, D., Jansen, S., Lewis, S.L., Swenson, N.G., Zanne, A.E., 2009. Towards a worldwide wood economics spectrum. Ecol. Lett. 12, 351-366. https://doi.org/10.1111/j.1461-0248.2009.01285.x

Chen, J.-L., Reynolds, J.F., 1997. A Coordination Model of Whole-plant Carbon Allocation in Relation to Water Stress. Ann. Bot. 80, 45-55. https://doi.org/10.1006/anbo.1997.0406 
Chen, B., Smith, S.L., 2018. CITRATE 1.0: Phytoplankton continuous trait-distribution model with one-dimensional physical transport applied to the Northwest Pacific. Geosci. Model Dev. 11, 467-495. https://doi.org/10.5194/gmd-11-467-2018

Chesson, P., 2000. General Theory of Competitive Coexistence in Spatially-Varying Environments. Theor. Popul. Biol. 58, 211-237. https://doi.org/10.1006/tpbi.2000.1486

Chesson, P., Gebauer, R.L.E., Schwinning, S., Huntly, N., Wiegand, K., Ernest, M.S.K., Sher, A., Novoplansky, A., Weltzin, J.F., 2004. Resource pulses, species interactions, and diversity maintenance in arid and semi-arid environments. Oecologia 141, 236-253. https://doi.org/10.1007/s00442-004-1551-1

Cicchetti, D. V., 1994. Guidelines, criteria, and rules of thumb for evaluating normed and stand ardized assessment instruments in psychology. Psychological assessment, 6 (4), 284-290

Cipriotti, P. A., Wiegand, T., Pütz, S., Bartoloni, N. J., Paruelo, J. M., 2016. Non-parametric up scaling of stochastic simulation models using transition matrices. Methods in Ecology and Evolution, 7 (3), 313-322

Clark, D.L., Wilson, M., Roberts, R., Dunwiddie, P.W., Stanley, A., Kaye, T.N., 2012. Plant traits - a tool for restoration? Appl. Veg. Sci. 15, 449-458. https://doi.org/10.1111/j.1654-109X.2012.01198.x

Clark, J.R., Lenton, T.M., Williams, H.T.P., Daines, S.J., 2013. Environmental selection and resource allocation determine spatial patterns in picophytoplankton cell size. Limnol. Oceanogr. 58, 1008-1022. https://doi.org/10.4319/lo.2013.58.3.1008

Colbach, N., Granger, S., Guyot, S.H.M., Mézière, D., 2014. A trait-based approach to explain weed species response to agricultural practices in a simulation study with a cropping system model. Agric. Ecosyst. Environ. 183, 197-204. https://doi.org/10.1016/j.agee.2013.11.013

Conti, S., O'Hagan, A., 2010. Bayesian Emulation of complex multi-output and dynamic computer models. Journal of Statistical Planning and Inference, 140 (3), 640-651

Copeland, S.M., Harrison, S.P., 2017. Community traits affect plant-plant interactions across climatic gradients. Oikos 126. https://doi.org/10.1111/oik.03376

Cornelissen, J.H.C., Lavorel, S., Garnier, E., Díaz, S., Buchmann, N., Gurvich, D.E., Reich, P.B., Steege, H. ter, Morgan, H.D., Heijden, M.G.A. van der, Pausas, J.G., Poorter, H., 2003. A handbook of protocols for standardised and easy measurement of plant functional traits worldwide. Aust. J. Bot. 51, 335. https://doi.org/10.1071/BT02124

Cox, D.R., Hinkley, D.V., Hinkley, D.V., 1979. Theoretical Statistics. Chapman and Hall/CRC. https://doi.org/10.1201/b14832

Crowther, T.W., Maynard, D.S., Crowther, T.R., Peccia, J., Smith, J.R., Bradford, M.A., 2014. Untangling the fungal niche: The trait-based approach. Front. Microbiol. 5, 1-12. https://doi.org/10.3389/fmicb.2014.00579

Cuddington, K., Fortin, M.-J., Gerber, L.R., Hastings, A., Liebhold, A., O’Connor, M., Ray, C., 2013. Process-based models are required to manage ecological systems in a changing world. Ecosphere 4, art20. https://doi.org/10.1890/ES12-00178.1

Cuesta, J.A., Delius, G.W., Law, R., 2018. Sheldon spectrum and the plankton paradox: two sides of the same coin-a traitbased plankton size-spectrum model. J. Math. Biol. 76, 67-96. https://doi.org/10.1007/s00285-017-1132-7

Dan, J., Yaalon, D.H., Koyumdjisky, H., Raz, Z., 1976. The soils of Israel:(With Map 1: 500,000).

Daws, M.I., Mullins, C.E., Burslem, D.F.R.P., Paton, S.R., Dalling, J.W., 2002. Topographic position affects the water regime in a semideciduous tropical forest in Panamá. Plant Soil 238, 79-89. https://doi.org/10.1023/A:1014289930621

DeAngelis, D.L., Grimm, V., 2014. Individual-based models in ecology after four decades. F1000Prime Rep. 6. https://doi.org/10.12703/P6-39

DeAngelis, D.L., Mooij, W.M., 2005. Individual-Based Modeling of Ecological and Evolutionary Processes. Annu. Rev. Ecol. Evol. Syst. 36, 147-168. https://doi.org/10.1146/annurev.ecolsys.36.102003.152644

Des Roches, Post, Turley, Bailey, Hendry, Kinnison, Schweitzer, Palkovacs, 2018. The ecological importance of intraspecific variation | [WWW Document]. Nat. Ecol. Evol. URL https://www.nature.com/articles/s41559-017-0402-5 (accessed 11.8.19).

DeWitt, T.J., Sih, A., Wilson, D.S., 1998. Costs and limits of phenotypic plasticity. Trends Ecol. Evol. 13, 77-81. https://doi.org/10.1016/S0169-5347(97)01274-3

Dey, S., Mukhopadhyay, T., Adhikari, S., 2017. Metamodel based high-fidelity stochastic analysis of composite laminates: A concise review with critical comparative assessment. Composite Structures, 171, 227-250

Diamond, S.E., Nichols, L.M., McCoy, N., Hirsch, C., Pelini, S.L., Sanders, N.J., Ellison, A.M., Gotelli, N.J., Dunn, R.R., 2012. A physiological trait-based approach to predicting the responses of species to experimental climate warming. Ecology 93, 2305-2312. https://doi.org/10.1890/11-2296.1 
Diaz, S., Cabido, M., 1997. Plant functional types and ecosystem function in relation to global change. J. Veg. Sci. 8, 463474. https://doi.org/10.2307/3237198

Diaz, S., Lavorel, S., de Bello, F., Quetier, F., Grigulis, K., Robson, T.M., 2007. Incorporating plant functional diversity effects in ecosystem service assessments. Proc. Natl. Acad. Sci. 104, 20684-20689. https://doi.org/10.1073/pnas.0704716104

Díaz, S., Kattge, J., Cornelissen, J.H.C., Wright, I.J., Lavorel, S., Dray, S., Reu, B., Kleyer, M., Wirth, C., Prentice, I.C., Garnier, E., Bönisch, G., Westoby, M., Poorter, H., Reich, P.B., Moles, A.T., Dickie, J., Gillison, A.N., Zanne, A.E., Chave, J., Wright, S.J., Sheremet'ev, S.N., Jactel, H., Christopher, B., Cerabolini, B., Pierce, S., Shipley, B., Kirkup, D., Casanoves, F., Joswig, J.S., Günther, A., Falczuk, V., Rüger, N., Mahecha, M.D., Gorné, L.D., 2015. The global spectrum of plant form and function. Nature 529, 1-17. https://doi.org/10.1038/nature16489

Dijkema, J., Koonce, J.E., Shillito, R.M., Ghezzehei, T.A., Berli, M., Ploeg, M.J. van der, Genuchten, M.T. van, 2018. Water Distribution in an Arid Zone Soil: Numerical Analysis of Data from a Large Weighing Lysimeter. Vadose Zone J. 17, 170035. https://doi.org/10.2136/vzj2017.01.0035

Drewniak, B., Gonzalez-Meler, M.A., 2017. Earth system model needs for including the interactive representation of nitrogen deposition and drought effects on forested ecosystems. Forests 8, 1-22. https://doi.org/10.3390/f8080267

Ecoutin, J.M., Simier, M., Albaret, J.J., Laë, R., Tito de Morais, L., 2010. Changes over a decade in fish assemblages exposed to both environmental and fishing constraints in the Sine Saloum estuary (Senegal). Estuar. Coast. Shelf Sci. 87, 284 292. https://doi.org/10.1016/J.ECSS.2010.01.009

Edmunds, P.J., Adjeroud, M., Baskett, M.L., Baums, I.B., Budd, A.F., Carpenter, R.C., Fabina, N.S., Fan, T.Y., Franklin, E.C., Gross, K., Han, X., Jacobson, L., Klaus, J.S., McClanahan, T.R., O’Leary, J.K., Van Oppen, M.J.H., Pochon, X., Putnam, H.M., Smith, T.B., Stat, M., Sweatman, H., Van Woesik, R., Gates, R.D., 2014. Persistence and change in community composition of reef corals through present, past, and future climates. PLoS One 9. https://doi.org/10.1371/journal.pone.0107525

Edwards, K.F., 2016. Community trait structure in phytoplankton: Seasonal dynamics from a method for sparse trait data. Ecology 97, 3441-3451. https://doi.org/10.1002/ecy.1581

Ellner, S., Shmida, A., 1981. Why are adaptations for long-range seed dispersal rare in desert plants? Oecologia 51, $133-144$. https://doi.org/10.1007/BF00344663

Enquist, B.J., Kerkhoff, A.J., Stark, S.C., Swenson, N.G., McCarthy, M.C., Price, C. a, 2007. A general integrative model for scaling plant growth, carbon flux, and functional trait spectra. Nature 449, 218-222. https://doi.org/10.1038/nature06061

Eppel, A., Shaked, R., Eshel, G., Barak, S., Rachmilevitch, S., 2014. Low induction of non-photochemical quenching and high photochemical efficiency in the annual desert plant Anastatica hierochuntica. Physiol. Plant. 151, 544-558. https://doi.org/10.1111/ppl.12146

Eshel, G., Shaked, R., Kazachkova, Y., Khan, A., Eppel, A., Cisneros, A., Acuna, T., Gutterman, Y., Tel-Zur, N., Rachmilevitch, S., Fait, A., Barak, S., 2017. Anastatica hierochuntica, an Arabidopsis Desert Relative, Is Tolerant to Multiple Abiotic Stresses and Exhibits Species-Specific and Common Stress Tolerance Strategies with Its Halophytic Relative, Eutrema (Thellungiella) salsugineum. Front. Plant Sci. 7. https://doi.org/10.3389/fpls.2016.01992

Evans, M.R., Grimm, V., Johst, K., Knuuttila, T., de Langhe, R., Lessells, C.M., Merz, M., O’Malley, M.A., Orzack, S.H., Weisberg, M., Wilkinson, D.J., Wolkenhauer, O., Benton, T.G., 2013. Do simple models lead to generality in ecology? Trends Ecol. Evol. 28, 578-583. https://doi.org/10.1016/j.tree.2013.05.022

Eviner, V.T., Chapin III, F.S., 2003. Functional Matrix: A Conceptual Framework for Predicting Multiple Plant Effects on Ecosystem Processes. Annu. Rev. Ecol. Evol. Syst. 34, 455-485. https://doi.org/10.1146/annurev.ecolsys.34.011802.132342

Falster, D.S., Brännström, ̊., Dieckmann, U., Westoby, M., 2011. Influence of four major plant traits on average height, leaf-area cover, net primary productivity, and biomass density in single-species forests: A theoretical investigation. J. Ecol. 99, 148-164. https://doi.org/10.1111/j.1365-2745.2010.01735.x

Follett, C.L., Dutkiewicz, S., Karl, D.M., Inomura, K., Follows, M.J., 2018. Seasonal resource conditions favor a summertime increase in North Pacific diatom-diazotroph associations. ISME J. 12, 1543-1557. https://doi.org/10.1038/s41396-017$0012-\mathrm{x}$

Follows, M.J., Dutkiewicz, S., 2011. Modeling Diverse Communities of Marine Microbes. Ann. Rev. Mar. Sci. 3, $427-451$. https://doi.org/10.1146/annurev-marine-120709-142848

Follows, M.J., Dutkiewicz, S., Grant, S., Chisholm, S.W., 2007. Emergent biogeography of microbial communities in a model ocean. Science 315, 1843-1846. https://doi.org/10.1126/science.1138544

Fonteyn, P.J., Mahall, B.E., 1978. Competition among desert perennials. Nature 275, 544-545.

https://doi.org/10.1038/275544a0 
Fort, F., Volaire, F., Guilioni, L., Barkaoui, K., Navas, M.L., Roumet, C., 2017. Root traits are related to plant water-use among rangeland Mediterranean species. Funct. Ecol. 31, 1700-1709. https://doi.org/10.1111/1365-2435.12888

Frenette-Dussault, C., Shipley, B., Meziane, D., Hingrat, Y., 2013. Trait-based climate change predictions of plant community structure in arid steppes. J. Ecol. 101, 484-492. https://doi.org/10.1111/1365-2745.12040

Friedman, J., Gunderman, N., Ellis, M., 1978. Water response of the hygrochastic skeletons of the true rose of Jericho (Anastatica hierochuntica L.). Oecologia 32, 289-301. https://doi.org/10.1007/BF00345108

Friedman, J., Stein, Z., 1980. The Influence of Seed-Dispersal Mechanisms on the Dispersion of Anastatica Hierochuntica (Cruciferae) in the Negev Desert, Israel. J. Ecol. 68, 43. https://doi.org/10.2307/2259242

Friedman, L. W., 1996. The Simulation Metamodel. New York, NY: Springer

Friedman, L. W., Pressman, I., 1988. The metamodel in simulation analysis: Can it be trusted? The Journal of the Operational research Society, $39(10), 939$

Funk, J.L., Larson, J.E., Ames, G.M., Butterfield, B.J., Cavender-Bares, J., Firn, J., Laughlin, D.C., Sutton-Grier, A.E., Williams, L., Wright, J., 2017. Revisiting the Holy Grail: using plant functional traits to understand ecological processes. Biol. Rev. 92, 1156-1173. https://doi.org/10.1111/brv.12275

Funk, J.L., Standish, R.J., Stock, W.D., Valladares, F., 2016. Plant functional traits of dominant native and invasive species in mediterranean-climate ecosystems. Ecology 97, 75-83. https://doi.org/10.1890/15-0974.1

Fyllas, N.M., Gloor, E., Mercado, L.M., Sitch, S., Quesada, C.A., Domingues, T.F., Galbraith, D.R., Torre-Lezama, A., Vilanova, E., Ramírez-Angulo, H., Higuchi, N., Neill, D.A., Silveira, M., Ferreira, L., Aymard C., G.A., Malhi, Y., Phillips, O.L., Lloyd, J., 2014. Analysing Amazonian forest productivity using a new individual and trait-based model (TFS v.1). Geosci. Model Dev. 7, 1251-1269. https://doi.org/10.5194/gmd-7-1251-2014

Gaedke, U., Klauschies, T., 2017. Analyzing the shape of observed trait distributions enables a data-based moment closure of aggregate models. Limnol. Oceanogr. Methods 15, 979-994. https://doi.org/10.1002/lom3.10218

Gamer, M., Lemon, J., Singh, I. F. P., 2019. irr: Various coefficients of interrater reliability and agreement. R package version 0.84.1. https://cran. r-project.org/package=irr

García, C., Borda-de-Água, L., 2017. Extended dispersal kernels in a changing world: insights from statistics of extremes. J. Ecol. 105, 63-74. https://doi.org/10.1111/1365-2745.12685

Gardner, R.H., Engelhardt, K.A.M., Elmore, A.J., Cadol, D., 2014. A traits-based model of species diversity. Ecol. Modell. 288, 178-194. https://doi.org/10.1016/j.ecolmodel.2014.06.006

Garnier, E., Navas, M.L., 2012. A trait-based approach to comparative functional plant ecology: Concepts, methods and applications for agroecology. A review, Agronomy for Sustainable Development. https://doi.org/10.1007/s13593-011-0036-y

Garnier, E., Shipley, B., 2001. A standardized protocol for the determination of specific leaf area and leaf dry matter content. Funct. Ecol. 15, 688-695. https://doi.org/10.1046/j.0269-8463.2001.00563.x

Garrard, G.E., McCarthy, M.A., Williams, N.S.G., Bekessy, S.A., Wintle, B.A., 2013. A general model of detectability using species traits. Methods Ecol. Evol. 4, 45-52. https://doi.org/10.1111/j.2041-210x.2012.00257.x

Gerlein-Safdi, C., Koohafkan, M.C., Chung, M., Rockwell, F.E., Thompson, S., Caylor, K.K., 2018. Dew deposition suppresses transpiration and carbon uptake in leaves. Agric. For. Meteorol. 259, 305-316.

https://doi.org/10.1016/j.agrformet.2018.05.015

Gervasi, S.S., Stephens, P.R., Hua, J., Searle, C.L., Xie, G.Y., Urbina, J., Olson, D.H., Bancroft, B.A., Weis, V., Hammond, J.I., Relyea, R.A., Blaustein, A.R., 2017. Linking ecology and epidemiology to understand predictors of multi-host responses to an emerging pathogen, the amphibian chytrid fungus. PLoS One 12, 1-23. https://doi.org/10.1371/journal.pone.0167882

Glibert, P.M., 2016. Margalef revisited: A new phytoplankton mandala incorporating twelve dimensions, including nutritional physiology. Harmful Algae 55, 25-30. https://doi.org/10.1016/j.hal.2016.01.008

Goldspink, C., 2000. Modelling social systems as complex: Towards a social simulation meta-model. Journal of Artificial Societies and Social Simulation, 3 (2), 1

Gong, Y., Lv, G., Guo, Z., Chen, Y., Cao, J., 2017. Influence of aridity and salinity on plant nutrients scales up from species to community level in a desert ecosystem. Sci. Rep. 7, 6811. https://doi.org/10.1038/s41598-017-07240-6

Gore, R., Diallo, S., Lynch, C., Padilla, J., 2017. Augmenting bottom-up metamodels with predicates. Journal of Artificial Societies and Social Simulation, 20 (1), 4 
Grassein, F., Lavorel, S., Till-Bottraud, I., 2014. The importance of biotic interactions and local adaptation for plant response to environmental changes: field evidence along an elevational gradient. Glob. Change Biol. 20, 1452-1460. https://doi.org/10.1111/gcb.12445

Gremer, J.R., Venable, D.L., 2014. Bet hedging in desert winter annual plants: optimal germination strategies in a variable environment. Ecol. Lett. 17, 380-387. https://doi.org/10.1111/ele.12241

Grigulis, K., Lavorel, S., Krainer, U., Legay, N., Baxendale, C., Dumont, M., Kastl, E., Arnoldi, C., Bardgett, R.D., Poly, F., Pommier, T., Schloter, M., Tappeiner, U., Bahn, M., Clément, J.-C., 2013. Relative contributions of plant traits and soil microbial properties to mountain grassland ecosystem services. J. Ecol. 101, 47-57. https://doi.org/10.1111/1365-2745.12014

Grime, J.P., 1977. Evidence for the Existence of Three Primary Strategies in Plants and Its Relevance to Ecological and Evolutionary Theory. Am. Nat. 111, 1169-1194. https://doi.org/10.1086/283244

Grime, J.P., 1974. Vegetation classification by reference to strategies. Nature 250, 26-31. https://doi.org/10.1038/250026a0

Grimm, V., 2005. Pattern-Oriented Modeling of Agent-Based Complex Systems: Lessons from Ecology. Science 310, $987-$ 991. https://doi.org/10.1126/science.1116681

Grimm, V., Berger, U., Bastiansen, F., Eliassen, S., Ginot, V., Giske, J., Goss-Custard, J., Grand, T., Heinz, S.K., Huse, G., Huth, A., Jepsen, J.U., Jørgensen, C., Mooij, W.M., Müller, B., Pe'er, G., Piou, C., Railsback, S.F., Robbins, A.M., Robbins, M.M., Rossmanith, E., Rüger, N., Strand, E., Souissi, S., Stillman, R.A., Vabø, R., Visser, U., DeAngelis, D.L., 2006. A standard protocol for describing individual-based and agent-based models. Ecol. Model. 198, 115-126. https://doi.org/10.1016/j.ecolmodel.2006.04.023

Grimm, V., Berger, U., 2016. Structural realism, emergence, and predictions in next-generation ecological modelling: Synthesis from a special issue. Ecol. Modell. 326, 177-187. https://doi.org/10.1016/j.ecolmodel.2016.01.001

Grimm, V., Railsback, S.F., 2005. Individual-based modeling and ecology. Princeton series in theoretical and computational biology, Princeton University Press, Princeton.

Grimm, V., Revilla, E., Berger, U., Jeltsch, F., Mooij, W.M., Railsback, S.F., Thulke, H.-H., Weiner, J., Wiegand, T., DeAngelis, D.L., 2005. Pattern-Oriented Modeling of Agent Based Complex Systems: Lessons from Ecology. Am. Assoc. Adv. Sci. 310, 987-991. https://doi.org/10.1126/science.1116681

Guiet, J., Aumont, O., Poggiale, J.C., Maury, O., 2016a. Effects of lower trophic level biomass and water temperature on fish communities: A modelling study. Prog. Oceanogr. 146, 22-37. https://doi.org/10.1016/j.pocean.2016.04.003

Guiet, J., Poggiale, J.C., Maury, O., 2016b. Modelling the community size-spectrum: recent developments and new directions. Ecol. Modell. 337, 4-14. https://doi.org/10.1016/j.ecolmodel.2016.05.015

Gutterman, Y., 1994. Strategies of seed dispersal and germination in plants inhabiting deserts. Bot. Rev. 60, 373-425. https://doi.org/10.1007/BF02857924

Gutterman, Y., 1997. Ibex diggings in the Negev Desert highlands of Israel as microhabitats for annual plants. Soil salinity, location and digging depth affecting variety and density of plant species. J. Arid Environ. 37, 665-681. https://doi.org/10.1006/jare.1997.0305

Gutterman, Y., 2000. Environmental factors and survival strategies of annual plant species in the Negev Desert, Israel. Plant Species Biol. 15, 113-125. https://doi.org/10.1046/j.1442-1984.2000.00032.x

Gutterman, Y., Ginott, S., 1994. Long-term protected "seed bank" in dry inflorescences of Asteriscus pygmaeus; achene dispersal mechanism and germination. J. Arid Environ. 26, 149-163. https://doi.org/10.1006/jare.1994.1019

Hagerthey, S.E., Newman, S., Xue, S., 2012. Periphyton-based transfer functions to assess ecological imbalance and management of a subtropical ombrotrophic peatland. Freshw. Biol. 57, 1947-1965. https://doi.org/10.1111/j.13652427.2012.02848.x

Haney, S.D., Siepielski, A.M., 2018. Tipping Points in Resource Abundance Drive Irreversible Changes in Community Structure. Am. Nat. 191, 000-000. https://doi.org/10.1086/697045

Hanski, I., Hansson, L., Henttonen, H., 1991. Specialist Predators, Generalist Predators, and the Microtine Rodent Cycle. J. Anim. Ecol. 60, 353. https://doi.org/10.2307/5465

Harfoot, M.B.J., Newbold, T., Tittensor, D.P., Emmott, S., Hutton, J., Lyutsarev, V., Smith, M.J., Scharlemann, J.P.W., Purves, D.W., 2014. Emergent Global Patterns of Ecosystem Structure and Function from a Mechanistic General Ecosystem Model. PLoS Biol. 12. https://doi.org/10.1371/journal.pbio.1001841

Harper, A.B., Cox, P.M., Friedlingstein, P., Wiltshire, A.J., Jones, C.D., Sitch, S., Mercado, L.M., Groenendijk, M., Robertson, E., Kattge, J., Bönisch, G., Atkin, O.K., Bahn, M., Cornelissen, J., Niinemets, Ü., Onipchenko, V., Peñuelas, J., Poorter, L., Reich, P.B., Soudzilovskaia, N.A., Van Bodegom, P., 2016. Improved representation of plant functional types and physi- 
ology in the Joint UK Land Environment Simulator (JULES v4.2) using plant trait information. Geosci. Model Dev. 9, 24152440. https://doi.org/10.5194/gmd-9-2415-2016

Harte, J., Shaw, R., 1995. Shifting Dominance Within a Montane Vegetation Community: Results of a Climate-Warming Experiment. Science 267, 876-880. https://doi.org/10.1126/science.267.5199.876

Hartig, F., 2019. DHARMa: Residual Diagnostics for Hierarchical (Multi-Level / Mixed) Regression Models. R package version 0.2.5. https://CRAN.R-project.org/package=DHARMa

Hartvig, M., Andersen, K.H., 2013. Coexistence of structured populations with size-based prey selection. Theor. Popul. Biol. 89, 24-33. https://doi.org/10.1016/j.tpb.2013.07.003

Hartvig, M., Andersen, K.H., Beyer, J.E., 2011. Food web framework for size-structured populations. J. Theor. Biol. 272, 113-122. https://doi.org/10.1016/j.jtbi.2010.12.006

Hastings, A., 1980. Disturbance, coexistence, history, and competition for space. Theor. Popul. Biol. 18, 363-373. https://doi.org/10.1016/0040-5809(80)90059-3

Hayat, S., Hayat, Q., Alyemeni, M.N., Wani, A.S., Pichtel, J., Ahmad, A., 2012. Role of proline under changing environments. Plant Signal. Behav. 7, 1456-1466. https://doi.org/10.4161/psb.21949

Heard, D., Dent, G., Schifeling, T., Banks, D., 2015. Agent-based models and microsimulation. Annual Review of Statistics and Its Application, 2 (1), 259-272

Hegazy, A.K., Kabiel, H.F., 2007. Significance of microhabitat heterogeneity in the spatial pattern and size-class structure of Anastatica hierochuntica L. Acta Oecologica 31, 332-342. https://doi.org/10.1016/j.actao.2007.01.006

Hérault, B., Bachelot, B., Poorter, L., Rossi, V., Bongers, F., Chave, J., Paine, C.E.T., Wagner, F., Baraloto, C., 2011. Functional traits shape ontogenetic growth trajectories of rain forest tree species. J. Ecol. 99, 1431-1440. https://doi.org/10.1111/j.1365-2745.2011.01883.x

Herben, T., Wildová, R., 2012. Community-level effects of plant traits in a grassland community examined by multispecies model of clonal plant growth. Ecol. Model., Modelling clonal plant growth: From Ecological concepts to Mathematics 234, 60-69. https://doi.org/10.1016/j.ecolmodel.2011.06.012

Herron, P.M., Martine, C.T., Latimer, A.M., Leicht-Young, S.A., 2007. Invasive plants and their ecological strategies: Prediction and explanation of woody plant invasion in New England. Divers. Distrib. 13, 633-644.

https://doi.org/10.1111/j.1472-4642.2007.00381.x

Hickman, A.E., Dutkiewicz, S., Williams, R.G., Follows, M.J., 2010. Modelling the effects of chromatic adaptation on phytoplankton community structure in the oligotrophic ocean. Mar. Ecol. Prog. Ser. 406, 1-17.

https://doi.org/10.3354/meps08588

Higgins, S.I., Richardson, D.M., 1998. Pine invasions in the southern hemisphere: modeling interactions between organism, enviroment and disturbance. Plant Ecol. 135, 79-93. https://doi.org/10.1023/a:1009760512895

Hill, A.J., Dawson, T.E., Shelef, O., Rachmilevitch, S., 2015. The role of dew in Negev Desert plants. Oecologia 178, $317-$ 327. https://doi.org/10.1007/s00442-015-3287-5

Hirt, M.R., Grimm, V., Li, Y., Rall, B.C., Rosenbaum, B., Brose, U., 2018. Bridging Scales: Allometric Random Walks Link Movement and Biodiversity Research. Trends Ecol. Evol. 33, 701-712. https://doi.org/10.1016/j.tree.2018.07.003

Hodgson, J.G., Wilson, P.J., Hunt, R., Grime, J.P., Thompson, K., 1999. Allocating C-S-R Plant Functional Types: A Soft Approach to a Hard Problem. Oikos 85, 282-294. https://doi.org/10.2307/3546494

Hof, A.R., Rodríguez-Castañeda, G., Allen, A.M., Jansson, R., Nilsson, C., 2017. Vulnerability of Subarctic and Arctic breeding birds: Ecol. Appl. 27, 219-234. https://doi.org/10.1002/eap.1434

Hoffmann, A.A., Merilä, J., 1999. Heritable variation and evolution under favourable and unfavourable conditions. Trends Ecol. Evol. 14, 96-101. https://doi.org/10.1016/S0169-5347(99)01595-5

Holzwarth, F., Rüger, N., Wirth, C., 2015. Taking a closer look: disentangling effects of functional diversity on ecosystem functions with a trait-based model across hierarchy and time. R. Soc. open Sci. 2, 140541. https://doi.org/10.1098/rsos.140541

Homolova, L., Schaepman, M.E., Lamarque, P., Clevers, J.G.P.W., De Bello, F., Thuiller, W., Lavorel, S., 2014. Comparison of remote sensing and plant trait-based modelling to predict ecosystem services in subalpine grasslands. Ecosphere 5, 1-29. https://doi.org/10.1890/ES13-00393.1

Houle, J.E., Andersen, K.H., Farnsworth, K.D., Reid, D.G., 2013. Emerging asymmetric interactions between forage and predator fisheries impose management trade-offsa. J. Fish Biol. 83, 890-904. https://doi.org/10.1111/jfb.12163 
Houle, J.E., de Castro, F., Cronin, M.A., Farnsworth, K.D., Gosch, M., Reid, D.G., 2016. Effects of seal predation on a modelled marine fish community and consequences for a commercial fishery. J. Appl. Ecol. 53, 54-63. https://doi.org/10.1111/1365-2664.12548

Howes, D.A., Abrahams, A.D., 2003. Modeling runoff and runon in a desert shrubland ecosystem, Jornada Basin, New Mexico. Geomorphology 53, 45-73. https://doi.org/10.1016/S0169-555X(02)00347-1

Howeth, J.G., Gantz, C.A., Angermeier, P.L., Frimpong, E.A., Hoff, M.H., Keller, R.P., Mandrak, N.E., Marchetti, M.P., Olden, J.D., Romagosa, C.M., Lodge, D.M., 2016. Predicting invasiveness of species in trade: climate match, trophic guild and fecundity influence establishment and impact of non-native freshwater fishes. Divers. Distrib. 22, 148-160. https://doi.org/10.1111/ddi.12391

Huebert, K.B., Pätsch, J., Hufnagl, M., Kreus, M., Peck, M.A., 2018. Modeled larval fish prey fields and growth rates help predict recruitment success of cod and anchovy in the North Sea. Mar. Ecol. Prog. Ser. 600, 111-126. https://doi.org/10.3354/meps12615

Hug Peter, D., Sardy, S., Diaz Rodriguez, J., Castella, E., Slaveykova, V.I., 2018. Modeling whole body trace metal concentrations in aquatic invertebrate communities: A trait-based approach. Environ. Pollut. 233, 419-428. https://doi.org/10.1016/j.envpol.2017.10.044

Hui, C., Richardson, D.M., Landi, P., Minoarivelo, H.O., Garnas, J., Roy, H.E., 2016. Defining invasiveness and invasibility in ecological networks. Biol. Invasions 18, 971-983. https://doi.org/10.1007/s10530-016-1076-7

Hutchinson, 1951. Copepodology for the Onithologist.

Hutchinson, G. E.,1957. Cold Spring Harbor Symposium on Quantitative Biology. Concluding Remarks.

Iooss, B., Lemaître, P., 2015. A Review on Global Sensitivity Analysis Methods, in: Dellino, G., Meloni, C. (Eds.), Uncertainty Management in Simulation-Optimization of Complex Systems: Algorithms and Applications, Operations Research/Computer Science Interfaces Series. Springer US, Boston, MA, pp. 101-122. https://doi.org/10.1007/978-1-4899$7547-8 \_5$

IPCC, 2018: Global Warming of $1.5^{\circ} \mathrm{C}$. An IPCC Special Report on the impacts of global warming of $1.5^{\circ} \mathrm{C}$ above preindustrial levels and related global greenhouse gas emission pathways, in the context of strengthening the global response to the threat of climate change, sustainable development, and efforts to eradicate poverty [Masson-Delmotte, V., P. Zhai, H.-O. Pörtner, D. Roberts, J. Skea, P.R. Shukla, A. Pirani, W. Moufouma-Okia, C. Péan, R. Pidcock, S. Connors, J.B.R. Matthews, Y. Chen, X. Zhou, M.I. Gomis, E. Lonnoy, T. Maycock, M. Tignor, and T. Waterfield (eds.)]. In Press., n.d. URL https://www.ipcc.ch/sr15/chapter/chapter-3/ (accessed 1.22.20).

Jabot, F., Faure, T., Dumoulin, N., 2013. EasyABC: performing efficient approximate Bayesian computation sampling schemes using R. Methods Ecol. Evol. 4, 684-687. https://doi.org/10.1111/2041-210X.12050

Jabot, F., 2010. A stochastic dispersal-limited trait-based model of community dynamics. J. Theor. Biol. 262, 650-661. https://doi.org/10.1016/j.jtbi.2009.11.004

Jabot F., Faure Th., Dumoulin N., Albert C., 2015. EasyABC: a R package to perform efficient approximate Bayesian computation sampling schemes. R package version 1.5. https://cran.r-project.org/web/packages/EasyABC/index.html

Jack, C.N., Friesen, M.L., Hintze, A., Sheneman, L., 2017. Third-party mutualists have contrasting effects on host invasion under the enemy-release and biotic-resistance hypotheses. Evol. Ecol. 31, 829-845. https://doi.org/10.1007/s10682-017$9912-5$

Jacobsen, N.S., Gislason, H., Andersen, K.H., 2014. The consequences of balanced harvesting of fish communities. Proc. R. Soc. B Biol. Sci. 281, 20132701-20132701. https://doi.org/10.1098/rspb.2013.2701

Jaffré, M., Le Galliard, J.F., 2016. Population viability analysis of plant and animal populations with stochastic integral projection models. Oecologia 182, 1031-1043. https://doi.org/10.1007/s00442-016-3704-4

James, J.J., Tiller, R.L., Richards, J.H., 2005. Multiple resources limit plant growth and function in a saline-alkaline desert community. J. Ecol. 93, 113-126. https://doi.org/10.1111/j.0022-0477.2004.00948.x

Jeffers, E.S., Bonsall, M.B., Froyd, C.A., Brooks, S.J., Willis, K.J., 2015. The relative importance of biotic and abiotic processes for structuring plant communities through time. J. Ecol. 103, 459-472. https://doi.org/10.1111/1365-2745.12365

Jeltsch, F., Moloney, K.A., Schurr, F.M., Köchy, M., Schwager, M., 2008. The state of plant population modelling in light of environmental change. Perspect. Plant Ecol. Evol. Syst. 9, 171-189. https://doi.org/10.1016/j.ppees.2007.11.004

Jennings, S., Collingridge, K., 2015. Predicting consumer biomass, size-structure, production, catch potential, responses to fishing and associated uncertainties in the world's marine ecosystems. PLoS One 10, 1-28.

https://doi.org/10.1371/journal.pone.0133794 
Jung, V., Albert, C.H., Violle, C., Kunstler, G., Loucougaray, G., Spiegelberger, T., 2014. Intraspecific trait variability mediates the response of subalpine grassland communities to extreme drought events. J. Ecol. 102, 45-53. https://doi.org/10.1111/1365-2745.12177

Kabiel, H.F., 2013. Genetic variations within and among populations of Anastatica hierochuntica at macroscale geographical range. Appl. Ecol. Environ. Res. 11, 343-354. https://doi.org/10.15666/aeer/1103_343354

Kalteh, A., Hjorth, P., Berndtsson, R., 2008. Review of the self-organizing map (SOM) approach in water resources: Analysis, modelling and application. Environmental Modelling \& Software, 23 (7), 835-845

Kattge, J., Diaz, S., Lavorel, S., Prentice, I.C., Leadley, P., Boenisch, G., Garnier, E., Westoby, M., Reich, P.B., Wright, I.J., Cornelissen, J.H.C., Violle, C., Harrison, S.P., Van Bodegom, P.M., Reichstein, M., Enquist, B.J., Soudzilovskaia, N.A., Ackerly, D.D., Anand, M., Atkin, O., Bahn, M., Baker, T.R., Baldocchi, D., Bekker, R., Blanco, C.C., Blonder, B., Bond, W.J., Bradstock, R., Bunker, D.E., Casanoves, F., Cavender-Bares, J., Chambers, J.Q., Chapin III, F.S., Chave, J., Coomes, D., Cornwell, W.K., Craine, J.M., Dobrin, B.H., Duarte, L., Durka, W., Elser, J., Esser, G., Estiarte, M., Fagan, W.F., Fang, J., Fernandez-Mendez, F., Fidelis, A., Finegan, B., Flores, O., Ford, H., Frank, D., Freschet, G.T., Fyllas, N.M., Gallagher, R. V., Green, W.A., Gutierrez, A.G., Hickler, T., Higgins, S.I., Hodgson, J.G., Jalili, A., Jansen, S., Joly, C.A., Kerkhoff, A. J., Kirkup, D., Kitajima, K., Kleyer, M., Klotz, S., Knops, J.M.H., Kramer, K., Kühn, I., Kurokawa, H., Laughlin, D., Lee, T.D., Leishman, M., Lens, F., Lenz, T., Lewis, S.L., Lloyd, J., Llusia, J., Louault, F., Ma, S., Mahecha, M.D., Manning, P., Massad, T., Medlyn, B.E., Messier, J., Moles, A.T., Müller, S.C., Nadrowski, K., Naeem, S., Niinemets, Ü., Nöllert, S., Nüske, A., Ogaya, R., Oleksyn, J., Onipchenko, V.G., Onoda, Y., Ordonez, J., Overbeck, G., Ozinga, W.A., Patino, S., Paula, S., Pausas, J.G., Penuelas, J., Phillips, O.L., Pillar, V., Poorter, H., Poorter, L., Poschlod, P., Prinzing, A., Proulx, R., Rammig, A., Reinsch, S., Reu, B., Sack, L., Salgado-Negret, B., Sardans, J., Shiodera, S., Shipley, B., Siefert, A., Sosinski, E., Soussana, J.-F., Swaine, E., Swenson, N., Thompson, K., Thornton, P., Waldram, M., Weiher, E., White, M., White, S., Wright, S.J., Yguel, B., Zaehle, S., Zanne, A.E., Wirth, C., 2011. TRY - a global database of plant traits. Glob. Chang. Biol. 17, 2905-2935. https://doi.org/10.1111/j.1365-2486.2011.02451.x

Kazakou, E., Violle, C., Roumet, C., Navas, M.-L., Vile, D., Kattge, J., Garnier, E., 2014. Are trait-based species rankings consistent across data sets and spatial scales? J. Veg. Sci. 25, 235-247. https://doi.org/10.1111/jvs.12066

Ke, P., Miki, T., Ding, T., 2015. Supporting Information The soil microbial community predicts the importance of plant traits in plant-soil feedback Po-Ju 329-341.

Kearney, M., Phillips, B.L., Tracy, C.R., Christian, K.A., Betts, G., Porter, W.P., 2008. Modelling species distributions without using species distributions: the cane toad in Australia under current and future climates. Ecography 31, 423-434. https://doi.org/10.1111/j.2008.0906-7590-05457.x

Kidron, G.J., 1999. Altitude dependent dew and fog in the Negev Desert, Israel. Agric. For. Meteorol. 96, 1-8. https://doi.org/10.1016/S0168-1923(99)00043-X

King, D., Roughgarden, J., 1982. Graded allocation between vegetative and reproductive growth for annual plants in growing seasons of random length. Theor. Popul. Biol. 22, 1-16. https://doi.org/10.1016/0040-5809(82)90032-6

Kiørboe, T., 2011. How zooplankton feed: Mechanisms, traits and trade-offs. Biol. Rev. 86, 311-339. https://doi.org/10.1111/j.1469-185X.2010.00148.x

Kleijnen, J. P., Sargent, R. G., 2000. A methodology for fitting and validating metamodels in simulation. European Journal of Operational research, $120(1), 14-29$

Kleyer, M., Bekker, R.M., Knevel, I.C., Bakker, J.P., Thompson, K., Sonnenschein, M., Poschlod, P., van Groenendael, J.M., Klimeš, L., Klimešová, J., Klotz, S., Rusch, G.M., Hermy, M., Adriaens, D., Boedeltje, G., Bossuyt, B., Dannemann, A., Endels, P., Götzenberger, L., Hodgson, J.G., Jackel, A.-K., Kühn, I., Kunzmann, D., Ozinga, W.A., Römermann, C., Stadler, M., Schlegelmilch, J., Steendam, H.J., Tackenberg, O., Wilmann, B., Cornelissen, J.H.C., Eriksson, O., Garnier, E., Peco, B., 2008. The LEDA Traitbase: a database of life-history traits of the Northwest European flora. J. Ecol. 96, 1266-1274. https://doi.org/10.1111/j.1365-2745.2008.01430.x

Knapp, A.K., 2002. Rainfall Variability, Carbon Cycling, and Plant Species Diversity in a Mesic Grassland. Science 298, 2202-2205. https://doi.org/10.1126/science.1076347

Kolbe, J.J., Kearney, M., Shine, R., 2010. Modeling the consequences of thermal trait variation for the cane toad invasion of Australia. Ecol. Appl. 20, 2273-2285. https://doi.org/10.1890/09-1973.1

Kolding, J., Jacobsen, N.S., Andersen, K.H., van Zwieten, P.A.M., 2016. Maximizing fisheries yields while maintaining community structure. Can. J. Fish. Aquat. Sci. 73, 644-655. https://doi.org/10.1139/cjfas-2015-0098

Koo, T. K. \& Li, M. Y., 2016. A guideline of selecting and reporting intraclass correlation coefficients for reliability research. Journal of Chiropractic Medicine, 15 (2), 155-163

Kraft, N.J.B., Crutsinger, G.M., Forrestel, E.J., Emery, N.C., 2014. Functional trait differences and the outcome of community assembly: an experimental test with vernal pool annual plants. Oikos 123, 1391-1399. https://doi.org/10.1111/oik.01311 
Kraft, N.J.B., Godoy, O., Levine, J.M., 2015. Plant functional traits and the multidimensional nature of species coexistence. Proc. Natl. Acad. Sci. 112, 797-802. https://doi.org/10.1073/pnas.1413650112

Kramer, P.J., 2019. Crop Reactions To Water And Temperature Stresses In Humid, Temperate Climates. CRC Press.

Küster, E.C., Kühn, I., Bruelheide, H., Klotz, S., 2008. Trait interactions help explain plant invasion success in the German flora. J. Ecol. 96, 860-868. https://doi.org/10.1111/j.1365-2745.2008.01406.x

Laliberté, E., Shipley, B., Norton, D.A., Scott, D., 2012. Which plant traits determine abundance under long-term shifts in soil resource availability and grazing intensity? J. Ecol. 100, 662-677. https://doi.org/10.1111/j.1365-2745.2011.01947.x

Lamarque, P., Lavorel, S., Mouchet, M., Quétier, F., 2014. Plant trait-based models identify direct and indirect effects of climate change on bundles of grassland ecosystem services. Proc. Natl. Acad. Sci. U. S. A. 111, 13751-6. https://doi.org/10.1073/pnas.1216051111

Larson, J.E., Funk, J.L., 2016. Regeneration: an overlooked aspect of trait-based plant community assembly models. J. Ecol. 104, 1284-1298. https://doi.org/10.1111/1365-2745.12613

Laubmeier, A.N., Wootton, K., Banks, J.E., Bommarco, R., Curtsdotter, A., Jonsson, T., Roslin, T., Banks, H.T., 2018. From theory to experimental design-Quantifying a trait-based theory of predator-prey dynamics. PLoS One 13, e0195919. https://doi.org/10.1371/journal.pone.0195919

Laughlin, D.C., 2014. Applying trait-based models to achieve functional targets for theory-driven ecological restoration. Ecol. Lett. 17, 771-784. https://doi.org/10.1111/ele.12288

Laughlin, D.C., Messier, J., 2015. Fitness of multidimensional phenotypes in dynamic adaptive landscapes. Trends Ecol. Evol. 30, 487-496. https://doi.org/10.1016/j.tree.2015.06.003

Laughlin, D.C., 2011. Nitrification is linked to dominant leaf traits rather than functional diversity. J. Ecol. 99, 1091-1099. https://doi.org/10.1111/j.1365-2745.2011.01856.x

Laughlin, D.C., Fulé, P.Z., Huffman, D.W., Crouse, J., Laliberté, E., 2011. Climatic constraints on trait-based forest assembly. J. Ecol. 99, 1489-1499. https://doi.org/10.1111/j.1365-2745.2011.01885.x

Laughlin, D.C., Joshi, C., 2015. Theoretical consequences of trait-based environmental filtering for the breadth and shape of the niche: New testable hypotheses generated by the Traitspace model. Ecol. Modell. 307, 10-21.

https://doi.org/10.1016/j.ecolmodel.2015.03.013

Laughlin, D.C., Joshi, C., Richardson, S.J., Peltzer, D.A., Mason, N.W.H., Wardle, D.A., 2015. Quantifying multimodal trait distributions improves trait-based predictions of species abundances and functional diversity. J. Veg. Sci. 26, 46-57. https://doi.org/10.1111/jvs.12219

Laughlin, D.C., Joshi, C., van Bodegom, P.M., Bastow, Z.A., Fulé, P.Z., 2012. A predictive model of community assembly that incorporates intraspecific trait variation. Ecol. Lett. 15, 1291-1299. https://doi.org/10.1111/j.1461-0248.2012.01852.x

Laughlin, D.C., Laughlin, D.E., 2013. Advances in modeling trait-based plant community assembly. Trends Plant Sci. 18, 584-593. https://doi.org/10.1016/j.tplants.2013.04.012

Laughlin, D.C., Strahan, R.T., Huffman, D.W., Sánchez Meador, A.J., 2017. Using trait-based ecology to restore resilient ecosystems: historical conditions and the future of montane forests in western North America. Restor. Ecol. 25, S135-S146. https://doi.org/10.1111/rec.12342

Lavorel, S., Garnier, E., 2002. Predicting changes in community composition and ecosystem functioning from plant traits: revisiting the Holy Grail. Funct. Ecol. 16, 545-556. https://doi.org/10.1046/j.1365-2435.2002.00664.x

Lavorel, S., McIntyre, S., Landsberg, J., Forbes, T.D.A., 1997. Plant functional classifications: from general groups to specific groups based on response to disturbance. Trends Ecol. Evol. 12, 474-478. https://doi.org/10.1016/S0169-5347(97)01219-6

Lavorel, S., Díaz, S., Cornelissen, J.H.C., Garnier, E., Harrison, S.P., Mcintyre, S., Pausas, J.G., Pérez-Harguindeguy, N., Roumet, C., Urcelay, C., 2007. Plant functional types: are we getting any closer to the Holy Grail? Terr. Ecosyst. a Chang. world 149-160. https://doi.org/10.1300/J035v16n03_03

Lavorel, S., Garnier, E., 2002. Predicting changes in community composition and ecosystem functioning from plant traits: revisiting the Holy Grail. Funct. Ecol. 16, 545-556. https://doi.org/10.1046/j.1365-2435.2002.00664.x

Lavorel, S., Grigulis, K., Lamarque, P., Colace, M.P., Garden, D., Girel, J., Pellet, G., Douzet, R., 2011. Using plant functional traits to understand the landscape distribution of multiple ecosystem services. J. Ecol. 99, 135-147. https://doi.org/10.1111/j.1365-2745.2010.01753.x

Lavorel, S., McIntyre, S., Landsberg, J., Forbes, T.D.A., 1997. Plant functional classifications: From general groups to specific groups based on response to disturbance. Trends Ecol. Evol. 12, 474-478. https://doi.org/10.1016/S0169-

5347(97)01219-6 
Lavorel, S., Storkey, J., Bardgett, R.D., De Bello, F., Berg, M.P., Le Roux, X., Moretti, M., Mulder, C., Pakeman, R.J., Díaz, S., Harrington, R., 2013. A novel framework for linking functional diversity of plants with other trophic levels for the quantification of ecosystem services. J. Veg. Sci. 24, 942-948. https://doi.org/10.1111/jvs.12083

Le Roux, X., Bouskill, N.J., Niboyet, A., Barthes, L., Dijkstra, P., Field, C.B., Hungate, B.A., Lerondelle, C., Pommier, T., Tang, J., Terada, A., Tourna, M., Poly, F., 2016. Predicting the responses of soil nitrite-oxidizers to multi-factorial global change: A trait-based approach. Front. Microbiol. 7, 1-13. https://doi.org/10.3389/fmicb.2016.00628

Leishman, M.R., 2001. Does the seed size/number trade-off model determine plant community structure? An assessment of the model mechanisms and their generality. Oikos 93, 294-302. https://doi.org/10.1034/j.1600-0706.2001.930212.x

Lentini, P.E., Bird, T.J., Griffiths, S.R., Godinho, L.N., Wintle, B.A., 2015. A global synthesis of survival estimates for microbats. Biol. Lett. 11. https://doi.org/10.1098/rsbl.2015.0371

Lepš, J., de Bello, F., Šmilauer, P., Doležal, J., 2011. Community trait response to environment: disentangling species turnover vs intraspecific trait variability effects. Ecography 34, 856-863. https://doi.org/10.1111/j.1600-0587.2010.06904.x

Levins, R., Culver, D., 1971. Regional Coexistence of Species and Competition between Rare Species. Proc. Natl. Acad. Sci. 68, 1246-1248. https://doi.org/10.1073/pnas.68.6.1246

Li, B., Wang, L., Kaseke, K.F., Vogt, R., Li, L., K. Seely, M., 2018. The impact of fog on soil moisture dynamics in the Namib Desert. Adv. Water Resour. 113, 23-29. https://doi.org/10.1016/j.advwatres.2018.01.004

Liancourt, P., Tielbörger, K., 2009. Competition and a short growing season lead to ecotypic differentiation at the two extremes of the ecological range. Funct. Ecol. 23, 397-404. https://doi.org/10.1111/j.1365-2435.2008.01497.x

Ligmann-Zielinska, A., Siebers, P.-O., Magliocca, N., Parker, D. C., Gri MM, V., Du, J., Cenek, M., Radchuk, V., Arbab, N. N., Li, S., Berger, U., Paudel, R., Robinson, D. T., Jankowski, P., An, L., Ye, X., 2020. One size does not fit: A roadmap of purpose-driven mixed-method pathways for sensitivity analysis of agent-based models. Journal of Artificial Societies and Social Simulation, 23 (1), 6

Lin, Y., Berger, U., Grimm, V., Ji, Q.-R., 2012. Differences between symmetric and asymmetric facilitation matter: exploring the interplay between modes of positive and negative plant interactions. J. Ecol. 100, 1482-1491. https://doi.org/10.1111/j.1365-2745.2012.02019.x

Litchman, E., de Tezanos Pinto, P., Klausmeier, C.A., Thomas, M.K., Yoshiyama, K., 2010. Linking traits to species diversity and community structure in phytoplankton, in: Fifty Years after the "Homage to Santa Rosalia": Old and New Paradigms on Biodiversity in Aquatic Ecosystems. Springer Netherlands, Dordrecht, pp. 15-28. https://doi.org/10.1007/978-90-4819908-2_3

Litchman, E., Klausmeier, C.A., 2008. Trait-Based Community Ecology of Phytoplankton. Annu. Rev. Ecol. Evol. Syst. 39, 615-639. https://doi.org/10.1146/annurev.ecolsys.39.110707.173549

Litchman, E., Klausmeier, C.A., Schofield, O.M., Falkowski, P.G., 2007. The role of functional traits and trade-offs in structuring phytoplankton communities: Scaling from cellular to ecosystem level. Ecol. Lett. 10, 1170-1181. https://doi.org/10.1111/j.1461-0248.2007.01117.x

Loarie, S.R., Duffy, P.B., Hamilton, H., Asner, G.P., Field, C.B., Ackerly, D.D., 2009. The velocity of climate change. Nature 462, 1052-1055. https://doi.org/10.1038/nature08649

Lochon, I., Colace, M.P., Devaux, C., Grigulis, K., Rettinger, R., Lavorel, S., 2018. Taxonomic and functional facets of the resilience to management of mown subalpine grasslands. Appl. Veg. Sci. 21, 636-646. https://doi.org/10.1111/avsc.12392

Loik, M.E., Breshears, D.D., Lauenroth, W.K., Belnap, J., 2004. A multi-scale perspective of water pulses in dryland ecosystems: climatology and ecohydrology of the western USA. Oecologia 141, 269-281. https://doi.org/10.1007/s00442-004$1570-\mathrm{y}$

Lortie, C.J., Brooker, R.W., Choler, P., Kikvidze, Z., Michalet, R., Pugnaire, F.I., Callaway, R.M., 2004. Rethinking plant community theory. Oikos 107, 433-438. https://doi.org/10.1111/j.0030-1299.2004.13250.x

Losapio, G., Schöb, C., 2017. Resistance of plant-plant networks to biodiversity loss and secondary extinctions following simulated environmental changes. Funct. Ecol. 31, 1145-1152. https://doi.org/10.1111/1365-2435.12839

Madin, J.S., Baird, A.H., Dornelas, M., Connolly, S.R., 2014. Mechanical vulnerability explains size-dependent mortality of reef corals. Ecol. Lett. 17, 1008-1015. https://doi.org/10.1111/ele.12306

Margalef, R., 1978. Life-forms of phytoplankton as survival alternatives in an unstable environment. Oceanol. Acta 1, 493509. https://doi.org/10.1007/BF00202661

Marjoram, P., Molitor, J., Plagnol, V., Tavaré, S., 2003. Markov chain Monte Carlo without likelihoods. Proc. Natl. Acad. Sci. 100, 15324-15328. https://doi.org/10.1073/pnas.0306899100 
Marteinsdóttir, B., 2014. Seed rain and seed bank reveal that seed limitation strongly influences plant community assembly in grasslands. PLoS One 9. https://doi.org/10.1371/journal.pone.0103352

Martonne, E. de, 1926. L'indice d'aridité. Bull. Assoc. Géographes Fr. 3, 3-5. https://doi.org/10.3406/bagf.1926.6321

Martorell, C., Martínez-López, M., 2014. Informed dispersal in plants: Heterosperma pinnatum (Asteraceae) adjusts its dispersal mode to escape from competition and water stress. Oikos 123, 225-231. https://doi.org/10.1111/j.16000706.2013.00715.x

Mason, N.W.H., Mouillot, D., Lee, W.G., Wilson, J.B., 2005. Functional richness, functional evenness and functional divergence: the primary components of functional diversity. Oikos 111, 112-118. https://doi.org/10.1111/j.00301299.2005.13886.x

Maury, O., Poggiale, J.-C.C., 2013. From individuals to populations to communities: A dynamic energy budget model of marine ecosystem size-spectrum including life history diversity. J. Theor. Biol. 324, 52-71. https://doi.org/10.1016/j.jtbi.2013.01.018

May, F., Grimm, V., Jeltsch, F., 2009. Reversed effects of grazing on plant diversity: the role of below-ground competition and size symmetry. Oikos 118, 1830-1843. https://doi.org/10.1111/j.1600-0706.2009.17724.x

Mayer, D.G., Butler, D.G., 1993. Statistical validation. Ecol. Model., Theoretical Modelling Aspects 68, 21-32. https://doi.org/10.1016/0304-3800(93)90105-2

Mayfield, M.M., Bonser, S.P., Morgan, J.W., Aubin, I., McNamara, S., Vesk, P.A., 2010. What does species richness tell us about functional trait diversity? Predictions and evidence for responses of species and functional trait diversity to land-use change. Glob. Ecol. Biogeogr. 19, 423-431. https://doi.org/10.1111/j.1466-8238.2010.00532.x

McAuliffe, J.R., 1988. Markovian Dynamics of Simple and Complex Desert Plant Communities. Am. Nat. 131, 459-490. https://doi.org/10.1086/284802

McDowell, N.G., Xu, C., 2017. Using traits to uncover tropical forest function. New Phytol. 214, 903-904. https://doi.org/10.1111/nph.14576

McGill, B., Enquist, B., Weiher, E., Westoby, M., 2006. Rebuilding community ecology from functional traits. Trends Ecol. Evol. 21, 178-185. https://doi.org/10.1016/j.tree.2006.02.002

McIntyre, S., Lavorel, S., Tremont, R.M., 1995. Plant Life-History Attributes: Their Relationship to Disturbance Response in Herbaceous Vegetation. J. Ecol. 83, 31-44. https://doi.org/10.2307/2261148

Melián, C.J., Baldó, F., Matthews, B., Vilas, C., González-Ortegón, E., Drake, P., Williams, R.J., 2014. Chapter Six - Individual Trait Variation and Diversity in Food Webs, Eco-Evolutionary Dynamics.

https://doi.org/http://dx.doi.org/10.1016/B978-0-12-801374-8.00006-2

Merow, C., Latimer, A.M., Silander, J.A.J., 2011. Can entropy maximization use functional traits to explain species abundance? A comprehensive evaluation. Ecology 92, 1523-1537. https://doi.org/10.1890/10-1174.1

Mertens, K. G., Lorscheid, I., Meyer, M., 2018. Using structural equation-based metamodeling for agent-based models. In W. K. V. Chan, A. D’Ambrogio, G. Zacharewicz, N. Mustafee, G. A. Wainer \& E. Page (Eds.), Proceedings of the 2017 Winter Simulation Conference, (pp. 1372-1382). San Diego, CA: IEEE

Messier, J., McGill, B.J., Enquist, B.J., Lechowicz, M.J., 2017. Trait variation and integration across scales: is the leaf economic spectrum present at local scales? Ecography 40, 685-697. https://doi.org/10.1111/ecog.02006

Meyer, K.M., Mooij, W.M., Vos, M., Hol, W.H.G., van der Putten, W.H., 2009. The power of simulating experiments. Ecol. Model. 220, 2594-2597. https://doi.org/10.1016/j.ecolmodel.2009.06.001

Michael, P. J., Steadman, K.J., Plummer, J.A., Vercoe, P., 2006. Sheep rumen digestion and transmission of weedy Malva parviflora seeds. Aust. J. Exp. Agric. 46, 1251-1256. https://doi.org/10.1071/EA05285

Michael, P. J., Steadman, K.J., Plummer, J.A., 2006. Climatic regulation of seed dormancy and emergence of diverse Malva parviflora populations from a Mediterranean-type environment. Seed Sci. Res. 16, 273-281. https://doi.org/10.1017/SSR2006254

Miki, T., Kondoh, M., 2002. Feedbacks between nutrient cycling and vegetation predict plant species coexistence and invasion. Ecol. Lett. 5, 624-633. https://doi.org/10.1046/j.1461-0248.2002.00347.x

Montalto, V., Rinaldi, A., Sarà, G., 2015. Life history traits to predict biogeographic species distributions in bivalves. Sci. Nat. 102. https://doi.org/10.1007/s00114-015-1313-4

Montgomery, D. C., 2009. Design and Analysis of Experiments. Hoboken, NJ: Wiley, 7 edn. 
Moor, H., 2017. Life history trade-off moderates model predictions of diversity loss from climate change. PLoS One 12, 121. https://doi.org/10.1371/journal.pone.0177778

Moorcroft, P. R., Hurtt, G. C. \& Pacala, S. W., 2001. A method for scaling vegetation dynamics: the ecosystem demography model (ED). Ecological Monographs, 71 (4), 557

Moore, A.D., Noble, I.R., 1990. An individualistic model of vegetation stand dynamics. J. Environ. Manage. 31, 61-81. https://doi.org/10.1016/S0301-4797(05)80015-5

Moran, E.V., Hartig, F., Bell, D.M., 2016. Intraspecific trait variation across scales: implications for understanding global change responses. Glob. Change Biol. 22, 137-150. https://doi.org/10.1111/gcb.13000

Mori, S., Yamaji, K., Ishida, A., Prokushkin, S.G., Masyagina, O. V., Hagihara, A., Hoque, A.T.M.R., Suwa, R., Osawa, A., Nishizono, T., Ueda, T., Kinjo, M., Miyagi, T., Kajimoto, T., Koike, T., Matsuura, Y., Toma, T., Zyryanova, O.A., Abaimov, A.P., Awaya, Y., Araki, M.G., Kawasaki, T., Chiba, Y., Umari, M., 2010. Mixed-power scaling of whole-plant respiration from seedlings to giant trees. Proc. Natl. Acad. Sci. 107, 1447-1451. https://doi.org/10.1073/pnas.0902554107

Morris, M.D., 1991. Factorial Sampling Plans for Preliminary Computational Experiments. Technometrics 33, $161-174$. https://doi.org/10.1080/00401706.1991.10484804

Mortensen, B., Abbott, K.C., Danielson, B., 2018. Defensive tradeoffs are not prerequisites to plant diversity in a two species model. Oikos 127, 63-72. https://doi.org/10.1111/oik.04300

Mott, J.J., McComb, A.J., 1974. Patterns in Annual Vegetation and Soil Microrelief in an Arid Region of Western Australia. J. Ecol. 62, 115-126. https://doi.org/10.2307/2258884

Nathan, R., Muller-Landau, H.C., 2000. Spatial patterns of seed dispersal, their determinants and consequences for recruitment. Trends Ecol. Evol. 15, 278-285. https://doi.org/10.1016/S0169-5347(00)01874-7

O'Farrell, S., Salguero-Gómez, R., van Rooij, J.M., Mumby, P.J., 2015. Disentangling trait-based mortality in species with decoupled size and age. J. Anim. Ecol. 84, 1446-1456. https://doi.org/10.1111/1365-2656.12399

O’Hagan, A., 2006. Bayesian analysis of compute R code outputs: A tutorial. Reliability Engineering \& System Safety, 91 (10-11), 1290-1300

Oakley, J., 2002. Bayesian inference for the uncertainty distribution of computer model output. Biometrika, 89 (4), $769-784$

Otfinowski, R., Kenkel, N.C., Dixon, P., Wilmshurst, J.F., 2007. Integrating climate and trait models to predict the invasiveness of exotic plants in Canada's Riding Mountain National Park. Can. J. Plant Sci. 87, 1001-1012. https://doi.org/10.4141/CJPS07117

Oudtshoorn, K. van R. van, Rooyen, M.W. van, 2013. Dispersal Biology of Desert Plants. Springer Science \& Business Media.

Ozgul, A., Bateman, A.W., English, S., Coulson, T., Clutton-Brock, T.H., 2014. Linking body mass and group dynamics in an obligate cooperative breeder. J. Anim. Ecol. 83, 1357-1366. https://doi.org/10.1111/1365-2656.12239

Ozgul, A., Coulson, T., Reynolds, A., Cameron, T.C., Benton, T.G., 2012. Population Responses to Perturbations: The Importance of Trait-Based Analysis Illustrated through a Microcosm Experiment. Am. Nat. 179, 582-594. https://doi.org/10.1086/664999

Pachepsky, E., Bown, J.L., Eberst, A., Bausenwein, U., Millard, P., Squire, G.R., Crawford, J.W., 2007. Consequences of intraspecific variation for the structure and function of ecological communities Part 2: Linking diversity and function. Ecol. Modell. 207, 277-285. https://doi.org/10.1016/j.ecolmodel.2007.05.005

Pachepsky, E., Crawford, J.W., Bown, J.L., Squire, G., 2001. Towards a general theory of biodiversity. Nature 410, $923-926$. https://doi.org/10.1038/35073563

Paleari, L., Movedi, E., Confalonieri, R., 2017. Trait-based model development to support breeding programs. A case study for salt tolerance and rice. Sci. Rep. 7, 1-11. https://doi.org/10.1038/s41598-017-04022-y

Papadopoulos, S., Azar, E., 2016. Integrating building performance simulation in agent-based modeling using regression surrogate models: A novel human-in-the-loop energy modeling approach. Energy and Buildings, 128 (656), 214-223

Paradis, E., Baillie, S.R., Sutherland, W.J., 2002. Modeling large-scale dispersal distances. Ecol. Model. 151, $279-292$. https://doi.org/10.1016/S0304-3800(01)00487-2

Pastor, A., Mariani, P., Erichsen, A.C., Hansen, F.T., Hansen, J.L.S., 2018. Modeling dispersal and spatial connectivity of macro-invertebrates in Danish waters: An agent-based approach. Reg. Stud. Mar. Sci. 20, 45-59.

https://doi.org/10.1016/J.RSMA.2018.03.005 
Pausas, J.G., 1999. Mediterranean vegetation dynamics: modelling problems and functional types. Plant Ecol. 140, $27-39$. https://doi.org/10.1023/A:1009752403216

Pavlick, R., Drewry, D.T., Bohn, K., Reu, B., Kleidon, A., 2012. The Jena Diversity-Dynamic Global Vegetation Model (JeDi-DGVM): a diverse approach to representing terrestrial biogeography and biogeochemistry based on plant functional trade-offs. Biogeosciences Discuss. 9, 4627-4726. https://doi.org/10.5194/bgd-9-4627-2012

Peeters, F., Straile, D., 2018. Trait selection and co-existence of phytoplankton in partially mixed systems: Trait based modelling and potential of an aggregated approach. PLoS One 13, 1-21. https://doi.org/10.1371/journal.pone.0194076

Pérez-Harguindeguy, N., Díaz, S., Garnier, E., Lavorel, S., Poorter, H., Jaureguiberry, P., Bret-Harte, M.S., Cornwell, W.K., Craine, J.M., Gurvich, D.E., Urcelay, C., Veneklaas, E.J., Reich, P.B., Poorter, L., Wright, I.J., Ray, P., Enrico, L., Pausas, J.G., De Vos, A.C., Buchmann, N., Funes, G., Quétier, F., Hodgson, J.G., Thompson, K., Morgan, H.D., Ter Steege, H., Van Der Heijden, M.G.A., Sack, L., Blonder, B., Poschlod, P., Vaieretti, M. V., Conti, G., Staver, A.C., Aquino, S., Cornelissen, J.H.C., 2013. New handbook for standardised measurement of plant functional traits worldwide. Aust. J. Bot. 61, 167-234. https://doi.org/10.1071/BT12225

Pérez-Ramos, I.M., Roumet, C., Cruz, P., Blanchard, A., Autran, P., Garnier, E., 2012. Evidence for a 'plant community economics spectrum' driven by nutrient and water limitations in a Mediterranean rangeland of southern France. J. Ecol. 100, 1315-1327. https://doi.org/10.1111/1365-2745.12000

Peters, R., Lin, Y., Berger, U., 2015. Machine Learning meets individual-based modelling: Self-organising feature maps for the analysis of below-ground competition among plants. Ecological Modelling, 326, 142-151

Pontarp, M., Wiens, J.J., 2017. The origin of species richness patterns along environmental gradients: uniting explanations based on time, diversification rate and carrying capacity. J. Biogeogr. 44, 722-735. https://doi.org/10.1111/jbi.12896

Pontarp, M., Petchey, O.L., 2016. Community trait overdispersion due to trophic interactions: Concerns for assembly process inference. Proc. R. Soc. B Biol. Sci. 283. https://doi.org/10.1098/rspb.2016.1729

Pope, J.G., Rice, J.C., Daan, N., Jennings, S., Gislason, H., 2006. Modelling an exploited marine fish community with 15 parameters - results from a simple size-based model. ICES J. Mar. Sci. 63, 1029-1044. https://doi.org/10.1016/j.icesjms.2006.04.015

Porter, W.P., Kearney, M., 2009. Size, shape, and the thermal niche of endotherms. Proc. Natl. Acad. Sci. 106, $19666-19672$. https://doi.org/10.1073/pnas.0907321106

Powney, G.D., Preston, C.D., Purvis, A., Van Landuyt, W., Roy, D.B., 2014. Can trait-based analyses of changes in species distribution be transferred to new geographic areas? Glob. Ecol. Biogeogr. 23, 1009-1018. https://doi.org/10.1111/geb.12189

Pöyry, J., Carvalheiro, L.G., Heikkinen, R.K., Kühn, I., Kuussaari, M., Schweiger, O., Valtonen, A., van Bodegom, P.M., Franzén, M., 2017. The effects of soil eutrophication propagate to higher trophic levels. Glob. Ecol. Biogeogr. 26, 18-30. https://doi.org/10.1111/geb.12521

Prentice, I.C., Bondeau, A., Cramer, W., Harrison, S.P., Hickler, T., Lucht, W., Sitch, S., Smith, B., Sykes, M.T., 2004. Dynamic Global Vegetation Modeling: Quantifying Terrestrial Ecosystem Responses to Large-Scale Environmental Change, in: Terrestrial Ecosystems in a Changing World. Springer, Berlin Heidelberg, pp. 175-192. https://doi.org/10.1007/978-3-54032730-1_15

Quétier, F., Lavorel, S., Liancourt, P., Thébault, A., Davies, I.D., 2011. Assessing long-term land-use legacies in subalpine grasslands by using a plant trait-based generic modelling framework. Plant Ecol. Divers. 4, 391-402. https://doi.org/10.1080/17550874.2011.629232

Quétier, F., Lavorel, S., Thuiller, W., Davies, I., 2007. Plant-trait-based modeling assessment of ecosystem-service sensitivity to land-use change. Ecol. Appl. 17, 2377-2386. https://doi.org/10.1890/06-0750.1

R Core Team (2018). R: A language and environment for statistical computing. R Foundation for Statistical Computing, Vienna, Austria. URL https://www.R-project.org/.

Radny, J., Meyer, K.M., 2018. The role of biotic factors during plant establishment in novel communities assessed with an agent-based simulation model. PeerJ 6, e5342. https://doi.org/10.7717/peerj.5342

Radny, J., van der Putten, W.H., Tielbörger, K., Meyer, K.M., 2018. Influence of seed size on performance of non-native annual plant species in a novel community at two planting densities. Acta Oecologica 92, 131-137. https://doi.org/10.1016/j.actao.2018.05.005

Railsback, S. F., Grimm, V., 2012. Agent-based and Individual-Based Modeling: A Practical Introduction. Princeton, NJ: Princeton University Press

Raunkiaer, C., 1934. The Life Forms of Plants and Statistical Plant Geography. Oxford University Press, Oxford. 
Read, Q.D., Henning, J.A., Sanders, N.J., 2017. Intraspecific variation in traits reduces ability of trait-based models to predict community structure. J. Veg. Sci. 28, 1070-1081. https://doi.org/10.1111/jvs.12555

Read, Q.D., Moorhead, L.C., Swenson, N.G., Bailey, J.K., Sanders, N.J., 2014. Convergent effects of elevation on functional leaf traits within and among species. Funct. Ecol. 28, 37-45. https://doi.org/10.1111/1365-2435.12162

Read, Q.D., Henning, J.A., Sanders, N.J., 2017. Intraspecific variation in traits reduces ability of trait-based models to predict community structure. J. Veg. Sci. 28, 1070-1081. https://doi.org/10.1111/jvs.12555

Rees, M., 1995. Community Structure in Sand Dune Annuals: Is Seed Weight a Key Quantity? J. Ecol. 83, 857-863. https://doi.org/10.2307/2261422

Reich, P.B., Wright, I.J., Cavender-Bares, J., Craine, J.M., Oleksyn, J., Westoby, M., Walters, M.B., 2003. The Evolution of Plant Functional Variation: Traits, Spectra, and Strategies. Int. J. Plant Sci. 164, S143-S164. https://doi.org/10.1086/374368

Reichman, O.J., 1984. Spatial and Temporal Variation of Seed Distributions in Sonoran Desert Soils. J. Biogeogr. 11, 1-11. https://doi.org/10.2307/2844771

Reick, C.H., Raddatz, T., Brovkin, V., Gayler, V., 2013. Representation of natural and anthropogenic land cover change in MPI-ESM. J. Adv. Model. Earth Syst. 459-482. https://doi.org/10.1002/jame.20022@10.1002/(ISSN)1942-2466.MPIESM1

Reu, B., Proulx, R., Bohn, K., Dyke, J.G., Kleidon, A., Pavlick, R., Schmidtlein, S., 2010. The role of climate and plant functional trade-offs in shaping global biome and biodiversity patterns. Glob. Ecol. Biogeogr. 20, 570-581. https://doi.org/10.1111/j.1466-8238.2010.00621.x

Reu, B., Zaehle, S., Proulx, R., Bohn, K., Kleidon, A., Pavlick, R., Schmidtlein, S., 2011. The role of plant functional tradeoffs for biodiversity changes and biome shifts under scenarios of global climatic change. Biogeosciences 8, 1255-1266. https://doi.org/10.5194/bg-8-1255-2011

Rewald, B., Eppel, A., Shelef, O., Hill, A., Degu, A., Friedjung, A., Rachmilevitch, S., 2012. Hot desert environments., in: Bell, E.M. (Ed.), Life at Extremes: Environments, Organisms and Strategies for Survival. CABI, Wallingford, pp. 196-218. https://doi.org/10.1079/9781845938147.0196

Reynolds, J.F., Kemp, P.R., Ogle, K., Fernández, R.J., 2004. Modifying the 'pulse-reserve' paradigm for deserts of North America: precipitation pulses, soil water, and plant responses. Oecologia 141, 194-210. https://doi.org/10.1007/s00442-004$1524-4$

Rinaldi, A., Montalto, V., Manganaro, A., Mazzola, A., Mirto, S., Sanfilippo, M., Sarà, G., Sara, G., 2014. Predictive mechanistic bioenergetics to model habitat suitability of shellfish culture in coastal lakes. Estuar. Coast. Shelf Sci. 144, 89-98. https://doi.org/10.1016/j.ecss.2014.04.013

Roelke, D.L., Spatharis, S., 2015a. Phytoplankton succession in recurrently fluctuating environments. PLoS One 10, 1-17. https://doi.org/10.1371/journal.pone.0121392

Roelke, D.L., Spatharis, S., 2015b. Phytoplankton assemblage characteristics in recurrently fluctuating environments. PLoS One 10, 1-25. https://doi.org/10.1371/journal.pone.0120673

Rosenfield, M.F., Müller, S.C., 2017. Predicting restored communities based on reference ecosystems using a trait-based approach. For. Ecol. Manage. 391, 176-183. https://doi.org/10.1016/j.foreco.2017.02.024

Sakschewski, B., von Bloh, W., Boit, A., Rammig, A., Kattge, J., Poorter, L., Peñuelas, J., Thonicke, K., 2015. Leaf and stem economics spectra drive diversity of functional plant traits in a dynamic global vegetation model. Glob. Chang. Biol. 21, 2711-2725. https://doi.org/10.1111/gcb.12870

Salecker, J., Sciaini, M., Meyer, K.M., Wiegand, K., 2019. The nlrx r package: A next-generation framework for reproducible NetLogo model analyses. Methods Ecol. Evol. 10, 1854-1863. https://doi.org/10.1111/2041-210X.13286

Salecker J., Sciaini M., 2019. nlrx: Setup, Run and Analyze 'NetLogo' Model Simulations from 'R' via 'XML'. R package version 0.2.0. https://CRAN.R-project.org/package=nlrx

Salguero-Gómez, R., Siewert, W., Casper, B.B., Tielbörger, K., 2012. A demographic approach to study effects of climate change in desert plants. Philos. Trans. R. Soc. B Biol. Sci. 367, 3100-3114. https://doi.org/10.1098/rstb.2012.0074

Salguero-Gómez, R., Violle, C., Gimenez, O., Childs, D., 2018. Delivering the promises of trait-based approaches to the needs of demographic approaches, and vice versa. Funct. Ecol. 32, 1424-1435. https://doi.org/10.1111/1365-2435.13148

Santini, L., Cornulier, T., Bullock, J.M., Palmer, S.C.F., White, S.M., Hodgson, J.A., Bocedi, G., Travis, J.M.J., 2016. A trait-based approach for predicting species responses to environmental change from sparse data: how well might terrestrial mammals track climate change? Glob. Chang. Biol. 22, 2415-2424. https://doi.org/10.1111/gcb.13271

Savage, V., Webb, C.T., Norberg, J., 2007. Biodiversity on Ecosystem Functioning. Theor. Biol. 247, 213-229. 
Scheffer, M., Baveco, J.M., DeAngelis, D.L., Rose, K.A., van Nes, E.H., 1995. Super-individuals a simple solution for modelling large populations on an individual basis. Ecol. Modell. 80, 161-170. https://doi.org/10.1016/0304-3800(94)00055-M

Scheiter, S., Langan, L., Higgins, S.I., 2013. Next-generation dynamic global vegetation models: learning from community ecology. New Phytol. 198, 957-969. https://doi.org/10.1111/nph.12210

Scheiter, S., Higgins, S.I., 2009. Impacts of climate change on the vegetation of Africa: An adaptive dynamic vegetation modelling approach. Glob. Chang. Biol. 15, 2224-2246. https://doi.org/10.1111/j.1365-2486.2008.01838.x

Scherer, C., Jeltsch, F., Grimm, V., Blaum, N., 2016. Merging trait-based and individual-based modelling: An animal functional type approach to explore the responses of birds to climatic and land use changes in semi-arid African savannas. Ecol. Modell. 326, 75-89. https://doi.org/10.1016/j.ecolmodel.2015.07.005

Schirpke, U., Kohler, M., Leitinger, G., Fontana, V., Tasser, E., Tappeiner, U., 2017. Future impacts of changing land-use and climate on ecosystem services of mountain grassland and their resilience. Ecosyst. Serv. 26, 79-94. https://doi.org/10.1016/j.ecoser.2017.06.008

Schleuning, M., Fründ, J., García, D., 2015. Predicting ecosystem functions from biodiversity and mutualistic networks: An extension of trait-based concepts to plant-animal interactions. Ecography 38, 380-392. https://doi.org/10.1111/ecog.00983

Schliep, E.M., Gelfand, A.E., Mitchell, R.M., Aiello-Lammens, M.E., Silander, J.A., 2018. Assessing the joint behaviour of species traits as filtered by environment. Methods Ecol. Evol. 9, 716-727. https://doi.org/10.1111/2041-210X.12901

Schlossberg, S., Chase, M.J., Griffin, C.R., 2018. Using species traits to predict detectability of animals on aerial surveys: Ecol. Appl. 28, 106-118. https://doi.org/10.1002/eap.1632

Schnedler-Meyer, N.A., Mariani, P., Kiørboe, T., 2016. The global susceptibility of coastal forage fish to competition by large jellyfish. Proc. R. Soc. B Biol. Sci. 283. https://doi.org/10.1098/rspb.2016.1931

Schrum, C., Alekseeva, I., St. John, M., 2006. Development of a coupled physical-biological ecosystem model ECOSMO. Part I: Model description and validation for the North Sea. J. Mar. Syst. 61, 79-99. https://doi.org/10.1016/j.jmarsys.2006.01.005

Schwinning, S., Weiner, J., 1998. Mechanisms determining the degree of size asymmetry in competition among plants. Oecologia 113, 447-455. https://doi.org/10.1007/s004420050397

Seifan, M., Seifan, T., Jeltsch, F., Tielbörger, K., 2012. Combined disturbances and the role of their spatial and temporal properties in shaping community structure. Perspect. Plant Ecol. Evol. Syst. 14, 217-229. https://doi.org/10.1016/j.ppees.2011.11.003

Seifan, M., Seifan, T., Schiffers, K., Jeltsch, F., Tielbörger, K., 2013. Beyond the Competition-Colonization Trade-Off: Linking Multiple Trait Response to Disturbance Characteristics. Am. Nat. 181, 151-160. https://doi.org/10.1086/668844

Shea, C.P., Bettoli, P.W., Potoka, K.M., Saylor, C.F., Shute, P.W., 2015. Use of Dynamic Occupancy Models to Assess the Response of Darters (Teleostei: Percidae) to Varying Hydrothermal Conditions in a Southeastern United States Tailwater. River Res. Appl. 31, 676-691. https://doi.org/10.1002/rra.2766

Shephard, S., Fung, T., Houle, J.E., Farnsworth, K.D., Reid, D.G., Rossberg, A.G., 2012. Size-selective fishing drives species composition in the Celtic Sea. ICES J. Mar. Sci. 69, 223-234. https://doi.org/10.1093/icesjms/fsr200

Shipley, B., Vile, D., Garnier, É., 2006. From Plant Traits to Plant Communities: A Statistical Mechanistic Approach to Biodiversity. Science 314, 812-814. https://doi.org/10.1126/science.1131344

Shipley, B., Laughlin, D.C., Sonnier, G., Otfinowski, R., 2011. A strong test of the maximum entropy model of trait- based community assembly. Ecology 92, 507-517. https://doi.org/10.1890/10-0394.1

Shocket, M.S., Strauss, A.T., Hite, J.L., Šljivar, M., Civitello, D.J., Duffy, M.A., Cáceres, C.E., Hall, S.R., 2018. Temperature Drives Epidemics in a Zooplankton-Fungus Disease System: A Trait-Driven Approach Points to Transmission Via Host Foraging. Am. Nat. 191, 000-000. https://doi.org/10.1086/696096

Siefert, A., Violle, C., Chalmandrier, L., Albert, C.H., Taudiere, A., Fajardo, A., Aarssen, L.W., Baraloto, C., Carlucci, M.B., Cianciaruso, M.V., de L. Dantas, V., de Bello, F., Duarte, L.D.S., Fonseca, C.R., Freschet, G.T., Gaucherand, S., Gross, N., Hikosaka, K., Jackson, B., Jung, V., Kamiyama, C., Katabuchi, M., Kembel, S.W., Kichenin, E., Kraft, N.J.B., Lagerström, A., Bagousse-Pinguet, Y.L., Li, Y., Mason, N., Messier, J., Nakashizuka, T., Overton, J.McC., Peltzer, D.A., Pérez-Ramos, I.M., Pillar, V.D., Prentice, H.C., Richardson, S., Sasaki, T., Schamp, B.S., Schöb, C., Shipley, B., Sundqvist, M., Sykes, M.T., Vandewalle, M., Wardle, D.A., 2015. A global meta-analysis of the relative extent of intraspecific trait variation in plant communities. Ecol. Lett. 18, 1406-1419. https://doi.org/10.1111/ele.12508

Smallegange, I.M., Ens, H.M., 2018. Trait-based predictions and responses from laboratory mite populations to harvesting in stochastic environments. J. Anim. Ecol. 87, 893-905. https://doi.org/10.1111/1365-2656.12802 
Smeti, E., Roelke, D.L., Tsirtsis, G., Spatharis, S., 2018. Species extinctions strengthen the relationship between biodiversity and resource use efficiency. Ecol. Modell. 384, 75-86. https://doi.org/10.1016/j.ecolmodel.2018.06.006

Smith, B., Prentice, I.C., Climate, M.T.S., Sykes, M.T., 2001. Representation of vegetation dynamics in the modelling of terrestrial ecosystems : comparing two contrasting approaches within European climate space. Glob. Ecol. Biogeogr. 10, 621-637. https://doi.org/10.1046/j.1466-822X.2001.00256.x

Smith, S.L., Merico, A., Hohn, S., Brandt, G., 2014. Sizing-up nutrient uptake kinetics: Combining a physiological trade-off with size-scaling of phytoplankton traits. Mar. Ecol. Prog. Ser. 511, 33-39. https://doi.org/10.3354/meps 10903

Snyder, R.E., 2006. Multiple risk reduction mechanisms: can dormancy substitute for dispersal? Ecol. Lett. 9, 1106-1114. https://doi.org/10.1111/j.1461-0248.2006.00962.x

Song, H.-S., Thomas, D.G., Stegen, J.C., Li, M., Liu, C., Song, X., Chen, X., Fredrickson, J.K., Zachara, J.M., Scheibe, T.D., 2017. Regulation-Structured Dynamic Metabolic Model Provides a Potential Mechanism for Delayed Enzyme Response in Denitrification Process. Front. Microbiol. 8, 1-12. https://doi.org/10.3389/fmicb.2017.01866

Sonnier, G., Shipley, B., Navas, M.L., 2010. Plant traits, species pools and the prediction of relative abundance in plant communities: A maximum entropy approach. J. Veg. Sci. 21, 318-331. https://doi.org/10.1111/j.1654-1103.2009.01145.x

Spasojevic, M.J., Suding, K.N., 2012. Inferring community assembly mechanisms from functional diversity patterns: the importance of multiple assembly processes. J. Ecol. 100, 652-661. https://doi.org/10.1111/j.1365-2745.2011.01945.x

Sterck, F., Markesteijn, L., Schieving, F., Poorter, L., 2011. Functional traits determine trade-offs and niches in a tropical forest community. Proc. Natl. Acad. Sci. 108, 20627-20632. https://doi.org/10.1073/pnas.1106950108

Sterck, F., Markesteijn, L., Toledo, M., Schieving, F., Poorter, L., 2014. Sapling performance along resource gradients drives tree species distributions within and across tropical forests. Ecology 95, 2514-2525. https://doi.org/10.1890/13-2377.1

Sterck, F., Schieving, F., 2011. Modelling functional trait acclimation for trees of different height in a forest light gradient: Emergent patterns driven by carbon gain maximization. Tree Physiol. 31, 1024-1037. https://doi.org/10.1093/treephys/tpr065

Strauss, A.T., Civitello, D.J., Cáceres, C.E., Hall, S.R., 2015. Success, failure and ambiguity of the dilution effect among competitors. Ecol. Lett. 18, 916-926. https://doi.org/10.1111/ele.12468

Suding, K.N., Goldstein, L.J., 2008. Testing the Holy Grail framework: Using functional traits to predict ecosystem change. New Phytol. 180, 559-562. https://doi.org/10.1111/j.1469-8137.2008.02650.x

Sun, X., Xiang, Y., Shi, Z., 2018. Estimating the hydraulic parameters of a confined aquifer based on the response of groundwater levels to seismic Rayleigh waves. Geophys. J. Int. 213, 919-930. https://doi.org/10.1093/gji/ggy036

Taffs, R., Aston, J.E., Brileya, K., Jay, Z., Klatt, C.G., McGlynn, S., Mallette, N., Montross, S., Gerlach, R., Inskeep, W.P., Ward, D.M., Carlson, R.P., 2009. In Silico approaches to study mass and energy flows in microbial consortia: A syntrophic case study. BMC Syst. Biol. 3, 1-16. https://doi.org/10.1186/1752-0509-3-114

Tanaka, Y., 2012. Trait response in communities to environmental change: Effect of interspecific competition and trait covariance structure. Theor. Ecol. 5, 83-98. https://doi.org/10.1007/s12080-010-0100-2

Taubert, F., Frank, K., Huth, A., 2012. A review of grassland models in the biofuel context. Ecol. Model. 245, 84-93. https://doi.org/10.1016/j.ecolmodel.2012.04.007

Team, R. C., 2018. R: A Language and Environment for Statistical Computing. R Foundation for Statistical Computing. Vienna. https://www. r-project.org

Tenan, S., Brambilla, M., Pedrini, P., Sutherland, C., 2017. Quantifying spatial variation in the size and structure of ecologically stratified communities. Methods Ecol. Evol. 8, 976-984. https://doi.org/10.1111/2041-210X.12719

Terseleer, N., Bruggeman, J., Lancelot, C., Gypens, N., 2014. Trait-based representation of diatom functional diversity in a plankton functional type model of the eutrophied southern north sea. Limnol. Oceanogr. 59, 1958-1972. https://doi.org/10.4319/1o.2014.59.6.1958

The Meteorologic Service Database, Israel Government Portal, 2020. URL: http://www.ims.gov.il/IMSEng/CLIMATE

Thiele, J. C., Kurth, W., Grimm, V., 2014. Facilitating parameter estimation and sensitivity analysis of agent-based models: A Cookbook using NetLogo and 'R'. Journal of Artificial Societies and Social Simulation, 17 (3), 11

Thomas, F.M., Vesk, P.A., 2017a. Are trait-growth models transferable? Predicting multi-species growth trajectories between ecosystems using plant functional traits. PLoS One 12, 1-19. https://doi.org/10.1371/journal.pone.0176959

Thomas, F.M., Vesk, P.A., 2017b. Growth races in The Mallee: Height growth in woody plants examined with a trait-based model. Austral Ecol. 42, 790-800. https://doi.org/10.1111/aec.12501 
Tielbörger, K., Kadmon, R., 2000. Temporal Environmental Variation Tips the Balance Between Facilitation and Interference in Desert Plants. Ecology 81, 1544-1553. https://doi.org/10.1890/0012-9658(2000)081[1544:TEVTTB]2.0.CO;2

Tielbörger, K., Valleriani, A., 2005. Can seeds predict their future? Germination strategies of density-regulated desert annuals. Oikos 111, 235-244. https://doi.org/10.1111/j.0030-1299.2005.14041.x

Tilman, D., 1994. Competition and Biodiversity in Spatially Structured Habitats. Ecology 75, 2-16. https://doi.org/10.2307/1939377

Treseder, K.K., 2016. Model behavior of arbuscular mycorrhizal fungi: Predicting soil carbon dynamics under climate change. Botany 1-25. https://doi.org/10.1099/vir.0.82772-0.Functional

Turcotte, M.M., Levine, J.M., 2016. Phenotypic Plasticity and Species Coexistence. Trends Ecol. Evol. 31, 803-813. https://doi.org/10.1016/j.tree.2016.07.013

Turnbull, L.A., Coomes, D., Hector, A., Rees, M., 2004. Seed mass and the competition/colonization trade-off: competitive interactions and spatial patterns in a guild of annual plants. J. Ecol. 92, 97-109. https://doi.org/10.1111/j.13652745.2004.00856.x

Turnbull, L.A., Rees, M., Crawley, M.J., 1999. Seed mass and the competition/colonization trade-off: a sowing experiment. J. Ecol. 87, 899-912. https://doi.org/10.1046/j.1365-2745.1999.00405.x

Turrens, J.F., 2003. Mitochondrial formation of reactive oxygen species. J. Physiol. 552, 335-344. https://doi.org/10.1111/j.1469-7793.2003.00335.x

Uller, T., 2008. Developmental plasticity and the evolution of parental effects. Trends Ecol. Evol. 23, 432-438. https://doi.org/10.1016/j.tree.2008.04.005

Urban, D. L., 2005. Modeling ecological processes across scales. Ecology, 86 (8), 1996-2006

Vale, C.G., Brito, J.C., 2015. Desert-adapted species are vulnerable to climate change: Insights from the warmest region on Earth. Glob. Ecol. Conserv. 4, 369-379. https://doi.org/10.1016/j.gecco.2015.07.012

Valladares, F., S. Joseph Wright, Eloisa Lasso, Kaoru Kitajima, Robert W. Pearcy, 2000. Valladares, F., Wright, S. J., Lasso, E., Kitajima, K., \& Pearcy, R. W. (2000). Plastic phenotypic response to light of 16 congeneric shrubs from a Panamanian rainforest.

Van Bellen, S., Mauquoy, D., Payne, R.J., Roland, T.P., Hughes, P.D.M., Daley, T.J., Loader, N.J., Street-Perrott, F.A., Rice, E.M., Pancotto, V.A., 2017. An alternative approach to transfer functions? Testing the performance of a functional trait-based model for testate amoebae. Palaeogeogr. Palaeoclimatol. Palaeoecol. 468, 173-183. https://doi.org/10.1016/j.palaeo.2016.12.005

Van Benthem, K.J., Froy, H., Coulson, T., Getz, L.L., Oli, M.K., Ozgul, A., 2017. Trait-demography relationships underlying small mammal population fluctuations. J. Anim. Ecol. 86, 348-358. https://doi.org/10.1111/1365-2656.12627

Van Bodegom, P.M., Douma, J.C., Witte, J.P.M.M., Ordoñez, J.C., Bartholomeus, R.P., Aerts, R., 2012. Going beyond limitations of plant functional types when predicting global ecosystem-atmosphere fluxes: exploring the merits of traits-based approaches. Glob. Ecol. Biogeogr. 21, 625-636. https://doi.org/10.1111/j.1466-8238.2011.00717.x

van der Putten, W.H., Bardgett, R.D., de Ruiter, P.C., Hol, W.H.G., Meyer, K.M., Bezemer, T.M., Bradford, M.A., Christensen, S., Eppinga, M.B., Fukami, T., Hemerik, L., Molofsky, J., Schädler, M., Scherber, C., Strauss, S.Y., Vos, M., Wardle, D.A., 2009. Empirical and theoretical challenges in aboveground-belowground ecology. Oecologia 161, 1-14. https://doi.org/10.1007/s00442-009-1351-8

Venable, D.L., Brown, J.S., 1988. The Selective Interactions of Dispersal, Dormancy, and Seed Size as Adaptations for Reducing Risk in Variable Environments. Am. Nat. 131, 360-384. https://doi.org/10.1086/284795

Venable, D.L., Flores-Martinez, A., Muller-Landau, H.C., Barron-Gafford, G., Becerra, J.X., 2008. Seed Dispersal of Desert Annuals. Ecology 89, 2218-2227. https://doi.org/10.1890/07-0386.1

Venable, D.L., Lawlor, L., 1980. Delayed germination and dispersal in desert annuals: Escape in space and time. Oecologia 46, 272-282. https://doi.org/10.1007/BF00540137

Verheijen, L.M., Brovkin, V., Aerts, R., Bönisch, G., Cornelissen, J.H.C., Kattge, J., Reich, P.B., Wright, I.J., Van Bodegom, P.M., 2013. Impacts of trait variation through observed trait-climate relationships on performance of an Earth system model: A conceptual analysis. Biogeosciences 10, 5497-5515. https://doi.org/10.5194/bg-10-5497-2013

Vindenes, Y., Edeline, E., Ohlberger, J., Langangen, Ø., Winfield, I.J., Stenseth, N.C., Vøllestad, L.A., 2014. Effects of Climate Change on Trait-Based Dynamics of a Top Predator in Freshwater Ecosystems. Am. Nat. 183, 243-256. https://doi.org/10.1086/674610 
Violle, C., Enquist, B.J., McGill, B.J., Jiang, L., Albert, C.H., Hulshof, C., Jung, V., Messier, J., 2012. The return of the variance: intraspecific variability in community ecology. Trends Ecol. Evol. 27, 244-252.

https://doi.org/10.1016/j.tree.2011.11.014

Violle, C., Navas, M.-L., Vile, D., Kazakou, E., Fortunel, C., Hummel, I., Garnier, E., 2007. Let the concept of trait be functional! Oikos 116, 882-892. https://doi.org/10.1111/j.0030-1299.2007.15559.x

Volis, S., Bohrer, G., 2013. Joint evolution of seed traits along an aridity gradient: seed size and dormancy are not two substitutable evolutionary traits in temporally heterogeneous environment. New Phytol. 197, 655-667.

https://doi.org/10.1111/nph.12024

Walker, A.P., Quaife, T., van Bodegom, P.M., De Kauwe, M.G., Keenan, T.F., Joiner, J., Lomas, M.R., MacBean, N., Xu, C., Yang, X., Woodward, F.I., 2017. The impact of alternative trait-scaling hypotheses for the maximum photosynthetic carboxylation rate (Vcmax) on global gross primary production. New Phytol. 215, 1370-1386. https://doi.org/10.1111/nph.14623

Wang, G. G., Shan, S., 2007. Review of metamodeling techniques in support of engineering design optimization. Journal of Mechanical Design, 129 (4), 370

Wang, P., Niu, G.-Y., Fang, Y.-H., Wu, R.-J., Yu, J.-J., Yuan, G.-F., Pozdniakov, S.P., Scott, R.L., 2018. Implementing Dynamic Root Optimization in Noah-MP for Simulating Phreatophytic Root Water Uptake. Water Resour. Res. 54, $1560-1575$. https://doi.org/10.1002/2017WR021061

Ward, D., 2009. The Biology of Deserts. Oxford University Press.

Warren, R.J., Lake, J.K., 2013. Trait plasticity, not values, best corresponds with woodland plant success in novel and manipulated habitats. J. Plant Ecol. 6, 201-210. https://doi.org/10.1093/jpe/rts035

Webb, C.T., Hoeting, J.A., Ames, G.M., Pyne, M.I., LeRoy Poff, N., 2010. A structured and dynamic framework to advance traits-based theory and prediction in ecology. Ecol. Lett. 13, 267-283. https://doi.org/10.1111/j.1461-0248.2010.01444.x

Weiner, J., Conte, P.T., 1981. Dispersal and neighborhood effects in an annual plant competition model. Ecol. Model. 13, 131-147. https://doi.org/10.1016/0304-3800(81)90048-X

Weiner, J., Damgaard, C., 2006. Size-asymmetric competition and size-asymmetric growth in a spatially explicit zone-ofinfluence model of plant competition. Ecol. Res. 21, 707-712. https://doi.org/10.1007/s11284-006-0178-6

Weiner, J., Stoll, P., Muller-Landau, H., Jasentuliyana, A., 2001. The Effects of Density, Spatial Pattern, and Competitive Symmetry on Size Variation in Simulated Plant Populations. Am. Nat. 158, 438-450. https://doi.org/10.1086/321988

Weiner, J., Wright, D.B., Castro, S., 1997. Symmetry of Below-Ground Competition between Kochia scoparia Individuals. Oikos 79, 85-91. https://doi.org/10.2307/3546093

Weiss, L., Pfestorf, H., May, F., Körner, K., Boch, S., Fischer, M., Müller, J., Prati, D., Socher, S.A., Jeltsch, F., 2014. Grazing response patterns indicate isolation of semi-natural European grasslands. Oikos 123, 599-612.

https://doi.org/10.1111/j.1600-0706.2013.00957.x

Weiss, L., Jeltsch, F., 2015. The response of simulated grassland communities to the cessation of grazing. Ecol. Modell. 303 , 1-11. https://doi.org/10.1016/j.ecolmodel.2015.02.002

Westoby, M., Falster, D.S., Moles, A.T., Vesk, P.A., Wright, I.J., 2002. Plant Ecological Strategies: Some Leading Dimensions of Variation Between Species. Annu. Rev. Ecol. Syst. 33, 125-159. https://doi.org/10.1146/annurev.ecolsys.33.010802.150452

Westoby, M., 1998. A leaf-height-seed (LHS) plant ecology strategy scheme. Plant Soil 199, 213-227. https://doi.org/10.1023/A:1004327224729

Wickham, H., 2016. ggplot2: Elegant Graphics for Data Analysis. Springer-Verlag New York.

Wickham, H., François, R., Henry, L., Müller, K., 2019. dplyr: A Grammar of Data Manipulation. R package version 0.8.3. https://CRAN.R-project.org/package=dplyr

Wieder, W.R., Grandy, A.S., Kallenbach, C.M., Taylor, P.G., Bonan, G.B., 2015. Representing life in the Earth system with soil microbial functional traits in the MIMICS model. Geosci. Model Dev. 8, 1789-1808. https://doi.org/10.5194/gmd-8$1789-2015$

Wiegand, T., Jeltsch, F., Hanski, I., Grimm, V., 2003. Using pattern-oriented modeling for revealing hidden information: a key for reconciling ecological theory and application. Oikos 100, 209-222. https://doi.org/10.1034/j.1600-0706.2003.12027.x

Wilensky, U., 1999. NetLogo. Evanston, IL: Center for connected learning and computer-based modeling, Northwestern University. 
Willis, S.G., Foden, W., Baker, D.J., Belle, E., Burgess, N.D., Carr, J.A., Doswald, N., Garcia, R.A., Hartley, A., Hof, C., Newbold, T., Rahbek, C., Smith, R.J., Visconti, P., Young, B.E., Butchart, S.H.M., 2015. Integrating climate change vulnerability assessments from species distribution models and trait-based approaches. Biol. Conserv. 190, 167-178. https://doi.org/10.1016/j.biocon.2015.05.001

Winemiller, K.O., Rose, K.A., 1992. Patterns of life-history in North American: Implications for Population Regulation. Can. J. Fish. Aquat. Sci. 49, 2196-2218. https://doi.org/10.1139/f92-242

Woodward, F.I., Lomas, M.R., 2004. Vegetation dynamics - simulating responses to climatic change. Biol. Rev. 79, 643670. https://doi.org/10.1017/S1464793103006419

Wright, I.J., Reich, P.B., Westoby, M., Ackerly, D.D., Baruch, Z., Bongers, F., Cavender-Bares, J., Chapin, T., Cornelissen, J.H.C., Diemer, M., Flexas, J., Garnier, E., Groom, P.K., Gulias, J., Hikosaka, K., Lamont, B.B., Lee, T., Lee, W., Lusk, C., Midgley, J.J., Navas, M.-L., Niinemets, U., Oleksyn, J., Osada, N., Poorter, H., Poot, P., Prior, L., Pyankov, V.I., Roumet, C., Thomas, S.C., Tjoelker, M.G., Veneklaas, E.J., Villar, R., 2004. The worldwide leaf economics spectrum. Nature 428, 821827. https://doi.org/10.1038/nature02403

Yang, Y., Wang, G., Xiong, Y., 2015. The contribution of intraspecific trait variability to plant community assembly patterns on the niche-neutral continuum. Pakistan J. Bot. 47, 1039-1050.

Yang, Y., Zhu, Q., Peng, C., Wang, H., Chen, H., 2015. From plant functional types to plant functional traits: A new paradigm in modelling global vegetation dynamics. Prog. Phys. Geogr. 39, 514-535. https://doi.org/10.1177/0309133315582018

Yenni, G., Adler, P.B., Morgan Ernest, S.K., 2012. Strong self-limitation promotes the persistence of rare species. Ecology 93, 456-461. https://doi.org/10.1890/11-1087.1

Zakharova, L., Meyer, K.M., Seifan, M., 2019. Trait-based modelling in ecology: A review of two decades of research. Ecol. Model. 407, 108703. https://doi.org/10.1016/j.ecolmodel.2019.05.008

Zhang, L., Hartvig, M., Knudsen, K., Andersen, K.H., 2014. Size-based predictions of food web patterns. Theor. Ecol. 7, 2333. https://doi.org/10.1007/s12080-013-0193-5

Zhang, L., Takahashi, D., Hartvig, M., Andersen, K.H., 2017. Food-web dynamics under climate change. Proc. R. Soc. B Biol. Sci. 284. https://doi.org/10.1177/1473325009337840

Zhang, L., Thygesen, U.H., Knudsen, K., Andersen, K.H., 2013. Trait diversity promotes stability of community dynamics. Theor. Ecol. 6, 57-69. https://doi.org/10.1007/s12080-012-0160-6

Zhu, Q., Iversen, C.M., Riley, W.J., Slette, I.J., Vander Stel, H.M., 2016. Root traits explain observed tundra vegetation nitrogen uptake patterns: Implications for trait-based land models. J. Geophys. Res. Biogeosciences 121, 3101-3112. https://doi.org/10.1002/2016JG003554

Zurell, D., Berger, U., Cabral, J.S., Jeltsch, F., Meynard, C.N., Münkemüller, T., Nehrbass, N., Pagel, J., Reineking, B., Schröder, B., Grimm, V., 2010. The virtual ecologist approach: simulating data and observers. Oikos 119, 622-635. https://doi.org/10.1111/j.1600-0706.2009.18284.x

Zwart, J.A., Solomon, C.T., Jones, S.E., 2015. Phytoplankton traits predict ecosystem function in a global set of lakes. Ecology 96, 2257-2264. https://doi.org/10.1890/14-2102.1 
Appendix 


\section{Appendix. Chapter 1.}

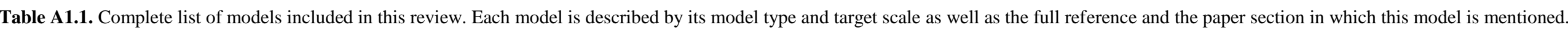

Section
1.1. Trait-based models on plant
growth, population dynamics,
and interactions
1.1. Trait-based models on plant
growth, population dynamics,
growth, population dynamics, and interactions

1.1. Trait-based models on plant growth, population dynamics, and interactions

1.1. Trait-based models on plant growth, population dynamics, and interactions

1.1. Trait-based models on plant growth, population dynamics, and interactions

1.1. Trait-based models on plant growth, population dynamics, and interactions

1.1. Trait-based models on plant growth, population dynamics, and interactions

1.1. Trait-based models on plant growth, population dynamics, and interactions

1.1. Trait-based models on plant growth, population dynamics, and interactions

1.1. Trait-based models on plan growth, population dynamics, and interactions

1.1. Trait-based models on plant growth, population dynamics, and interactions

1.1. Trait-based models on plan growth, population dynamics, and interactions

\section{Reference}

Sterck, F., Schieving, F., 2011. Modelling functional trait acclimation for trees of different height in a forest light gradient:

Emergent patterns driven by carbon gain maximization. Tree Physiol. 31, 1024-1037. https://doi.org/10.1093/treephys/tpr065

Ali, A.A., Medlyn, B.E., Aubier, T.G., Crous, K.Y., Reich, P.B., 2015. Elevated carbon dioxide is predicted to promote coexistence among competing species in a trait-based model. Ecol. Evol. 5, 4717-4733. https://doi.org/10.1002/ece3.1733

Ali, A.A., Medlyn, B.E., Crous, K.Y., Reich, P.B., 2013. A trait-based ecosystem model suggests that long-term responsiveness to rising atmospheric $\mathrm{CO}_{2}$ concentration is greater in slow-growing than fast-growing plants. Functional Ecology 27, 10111022. https://doi.org/10.1111/1365-2435.12102

Aubry-Kientz, M., Rossi, V., Boreux, J.-J., Hérault, B., 2015. A joint individual-based model coupling growth and mortality reveals that tree vigor is a key component of tropical forest dynamics. Ecology and Evolution 5, 2457-2465. https://doi.org/10.1002/ece3.1532

Bown, J.L., Pachepsky, E., Eberst, A., Bausenwein, U., Millard, P., Squire, G.R., Crawford, J.W., 2007. Consequences of intraspecific variation for the structure and function of ecological communities. Part 1 . Model development and predicted patterns of diversity. Ecol. Modell. 207, 264-276. https://doi.org/10.1016/j.ecolmodel.2007.05.004

Chavana-Bryant, C., Malhi, Y., Wu, J., Asner, G.P., Anastasiou, A., Enquist, B.J., Cosio Caravasi, E.G., Doughty, C.E., Saleska, S.R., Martin, R.E., Gerard, F.F., 2017. Leaf aging of Amazonian canopy trees as revealed by spectral and physiochemical measurements. New Phytol. 214, 1049-1063. https://doi.org/10.1111/nph.13853

Enquist, B.J., Kerkhoff, A.J., Stark, S.C., Swenson, N.G., McCarthy, M.C., Price, C. a, 2007. A general integrative model for scaling plant growth, carbon flux, and functional trait spectra. Nature 449, 218-222. https://doi.org/10.1038/nature06061

Fort, F., Volaire, F., Guilioni, L., Barkaoui, K., Navas, M.-L., Roumet, C., 2017. Root traits are related to plant water-use among rangeland Mediterranean species. Functional Ecology 31, 1700-1709. https://doi.org/10.1111/1365-2435.12888

Fyllas, N.M., Gloor, E., Mercado, L.M., Sitch, S., Quesada, C.A., Domingues, T.F., Galbraith, D.R., Torre-Lezama, A., Vilanova, E., Ramírez-Angulo, H., Higuchi, N., Neill, D.A., Silveira, M., Ferreira, L., Aymard C., G.A., Malhi, Y., Phillips, O.L., Lloyd, J., 2014. Analysing Amazonian forest productivity using a new individual and trait-based model (TFS v.1). Geosci. Model Dev. 7, 1251-1269. https://doi.org/10.5194/gmd-7-1251-2014

Hérault, B., Bachelot, B., Poorter, L., Rossi, V., Bongers, F., Chave, J., Paine, C.E.T., Wagner, F., Baraloto, C., 2011. Functional traits shape ontogenetic growth trajectories of rain forest tree species. J. Ecol. 99, 1431-1440. https://doi.org/10.1111/j.1365-2745.2011.01883.x et al., 2011.

Paleari, L., Movedi, E., Confalonieri, R., 2017. Trait-based model development to support breeding programs. A case study for salt tolerance and rice. Sci. Rep. 7, 1-11. https://doi.org/10.1038/s41598-017-04022-y

Taubert, F., Frank, K., Huth, A., 2012. A review of grassland models in the biofuel context. Ecol. Modell. 245, 84-93. https://doi.org/10.1016/j.ecolmodel.2012.04.007

$\begin{array}{ll}\text { Model type } & \text { Target sca } \\ \text { equation-based } & \text { individual }\end{array}$

equation-based

ecosystem

equation-based

ecosystem

individual-based + statistical

individual-based

statistica

individual

equation-based

individual

equation-based

species

equation-based

ecosystem, community

statistical

individual, species

individual

individual-based

ecosystem,

community 
1.1. Trait-based models on plant growth, population dynamics, and interactions

1.1. Trait-based models on plant growth, population dynamics, and interactions

1.1. Trait-based models on plant growth, population dynamics, and interactions

1.2. Trait-based models on species distributions

1.2. Trait-based models on species distributions

1.2. Trait-based models on species distribution

1.2. Trait-based models on species distributions

1.2. Trait-based models on species distributions

1.2. Trait-based models on species distributions

1.2. Trait-based models on species distributions

1.2. Trait-based models on species distributions

1.2. Trait-based models on species distributions
Thomas, F.M., Vesk, P.A., 2017b. Growth races in The Mallee: Height growth in woody plants examined with a trait-based model. Austral Ecol. 42, 790-800. https://doi.org/10.1111/aec.1250

Adler, P.B., Salguero-Gomez, R., Compagnoni, A., Hsu, J.S., Ray-Mukherjee, J., Mbeau-Ache, C., Franco, M., 2014. Functional traits explain variation in plant life history strategies. Proceedings of the National Academy of Sciences 111, 740-745. https://doi.org/10.1073/pnas.1315179111

Jaffré, M., Le Galliard, J.-F., 2016. Population viability analysis of plant and animal populations with stochastic integral projection models. Oecologia 182, 1031-1043. https://doi.org/10.1007/s00442-016-3704-4

Laughlin, D.C., Joshi, C., 2015. Theoretical consequences of trait-based environmental filtering for the breadth and shape of the niche: New testable hypotheses generated by the Traitspace model. Ecological Modelling 307, 10-21.

https://doi.org/10.1016/j.ecolmodel.2015.03.013

Douma, J.C., Witte, J.-P.M., Aerts, R., Bartholomeus, R.P., Ordoñez, J.C., Venterink, H.O., Wassen, M.J., van Bodegom, P.M., statistical 2012. Towards a functional basis for predicting vegetation patterns; incorporating plant traits in habitat distribution models

Ecography 35, 294-305. https://doi.org/10.1111/j.1600-0587.2011.07140.x

Garrard, G.E., McCarthy, M.A., Williams, N.S.G., Bekessy, S.A., Wintle, B.A., 2013. A general model of detectability using species traits. Methods Ecol. Evol. 4, 45-52. https://doi.org/10.1111/j.2041-210x.2012.00257.x

Laughlin, D.C., Joshi, C., van Bodegom, P.M., Bastow, Z.A., Fulé, P.Z., 2012. A predictive model of community assembly that incorporates intraspecific trait variation. Ecol. Lett. 15, 1291-1299. https://doi.org/10.1111/j.1461-0248.2012.01852.x

Laughlin, D.C., Laughlin, D.E., 2013. Advances in modeling trait-based plant community assembly. Trends Plant Sci. 18, 584593. https://doi.org/10.1016/j.tplants.2013.04.012

Powney, G.D., Preston, C.D., Purvis, A., Van Landuyt, W., Roy, D.B., 2014. Can trait-based analyses of changes in species distribution be transferred to new geographic areas? Glob. Ecol. Biogeogr. 23, 1009-1018. https://doi.org/10.1111/geb.12189

Rosenfield, M.F., Müller, S.C., 2017. Predicting restored communities based on reference ecosystems using a trait-based approach. For. Ecol. Manage. 391, 176-183. https://doi.org/10.1016/j.foreco.2017.02.024

Shipley, B., Laughlin, D.C., Sonnier, G., Otfinowski, R., 2011. A strong test of the maximum entropy model of trait- based community assembly. Ecology 92, 507-517. https://doi.org/10.1890/10-0394.1

Sonnier, G., Shipley, B., Navas, M.-L., 2010. Plant traits, species pools and the prediction of relative abundance in plant communities: a maximum entropy approach. Journal of Vegetation Science 21, 318-331. https://doi.org/10.1111/j.1654

1103.2009.01145.x 


Section

cies distributions

1.2. Trait-based models on species distributions

1.2. Trait-based models on species distributions

1.2. Trait-based models on species distribution

1.3. Trait-based models of community assembly

1.3. Trait-based models of community assembly

1.3. Trait-based models of community assembly

1.3. Trait-based models of community assembly

1.3. Trait-based models of community assembly

1.3. Trait-based models of community assembly

1.3. Trait-based models of community assembly

1.3. Trait-based models of community assembly

1.3. Trait-based models of community assembly
Sterck, F., Markesteijn, L., Toledo, M., Schieving, F., Poorter, L., 2014. Sapling performance along resource gradients drives

Willis, S.G., Foden, W., Baker, D.J., Belle, E., Burgess, N.D., Carr, J.A., Doswald, N., Garcia, R.A., Hartley, A., Hof, C.

conceptual

community Newbold, T., Rahbek, C. Smith, R. J., Visconti, P., Young, B.E., Butchart, S.H.M., 2015. Integrating climate change vulnerability assessments from species distribution models and trait-based approaches. Biological Conservation 190, 167-178. https://doi.org/10.1016/j.biocon.2015.05.00

Abrego, N., Norberg, A., Ovaskainen, O., 2017. Measuring and predicting the influence of traits on the assembly processes of wood-inhabiting fungi. Journal of Ecology 105, 1070-1081. https://doi.org/10.1111/1365-2745.12722

Bhaskar, R., Dawson, T.E., Balvanera, P., 2014. Community assembly and functional diversity along succession postmanagement. Functional Ecology 28, 1256-1265. https://doi.org/10.1111/1365-2435.12257

Chauvet, M., Kunstler, G., Roy, J., Morin, X.,2017. Using a forest dynamics model to link community assembly processes and traits structure. Functional Ecology 31, 1452-1461. https://doi.org/10.1111/1365-2435.12847

Kraft, N.J.B., Godoy, O, Levine, J.M., 2015. Plant functional traits and the multidimensional nature of species coexistence. Proceedings of the National Academy of Sciences 112, 797-802. https://doi.org/10.1073/pnas.1413650112

Laughlin, D.C., 2011. Nitrification is linked to dominant leaf traits rather than functional diversity. J. Ecol. 99, 1091-1099. https://doi.org/10.1111/j.1365-2745.2011.01856.x

Laughlin, D.C., 2014. Applying trait-based models to achieve functional targets for theory-driven ecological restoration. Ecol. Lett. 17, 771-784. https://doi.org/10.1111/ele.12288

Laughlin, D.C., Fulé, P.Z., Huffman, D.W., Crouse, J., Laliberté, E., 2011. Climatic constraints on trait-based forest assembly: Climatic constraints on forest assembly. Journal of Ecology 99, 1489-1499. https://doi.org/10.1111/j.1365-2745.2011.01885.x

Laughlin, D.C., Joshi, C., Richardson, S.J., Peltzer, D.A., Mason, N.W.H., Wardle, D.A., 2015. Quantifying multimodal trait distributions improves trait-based predictions of species abundances and functional diversity. J. Veg. Sci. 26, 46-57. https://doi.org/10.1111/jvs.12219

Laughlin, D.C. Strahan, R. T. Huffman, D.W., Sánchez Meador, A.J., 2017. Using trait-based ecology to restore resilient ecosystems: historical conditions and the future of montane forests in western North America. Restor. Ecol. 25, S135-S146. https://doi.org/10.1111/rec.12342

Losapio, G., Schöb, C., 2017. Resistance of plant-plant networks to biodiversity loss and secondary extinctions following simulated environmental changes. Functional Ecology 31, 1145-1152. https://doi.org/10.1111/1365-2435.12839 community

community

community

equation-based

community

statistical

global

equation-based +

statistical

community

statistical

community

equation-based +

global

statistical

equation-based + statistical

community

(a)

community 


Section
1.3. Trait-based models of com-
munity assembly
1.3. Trait-based models of com-
munity assembly
1.3. Trait-based models of com-
munity assembly
1.3. Trait-based models of com-
munity assembly

1.3. Trait-based models of community assembly

1.3. Trait-based models of community assembly

1.3. Trait-based models of community assembly

1.3. Trait-based models of community assembly

1.3. Trait-based models of community assembly

1.3. Trait-based models of community assembly

1.4. Trait-based models of community dynamics

1.4. Trait-based models of community dynamics

1.4. Trait-based models of community dynamics

\section{Reference}

Marteinsdóttir, B., 2014. Seed rain and seed bank reveal that seed limitation strongly influences plant community assembly in grasslands. PLoS One 9. https://doi.org/10.1371/journal.pone.0103352

Pachepsky, E., Bown, J.L., Eberst, A., Bausenwein, U., Millard, P., Squire, G.R., Crawford, J.W., 2007. Consequences of intraspecific variation for the structure and function of ecological communities Part 2: Linking diversity and function. Ecological Modelling 207, 277-285. https://doi.org/10.1016/j.ecolmodel.2007.05.005

Schliep, E.M., Gelfand, A.E., Mitchell, R.M., Aiello-Lammens, M.E., Silander, J.A., 2018. Assessing the joint behaviour of species traits as filtered by environment. Methods in Ecology and Evolution 9, 716-727. https://doi.org/10.1111/2041210X.12901

Shipley, B., Vile, D., Garnier, E., 2006. From Plant Traits to Plant Communities: A Statistical Mechanistic Approach to Biodiversity. Science. $314,812-814$. https://doi.org/10.1126/science.1131344

Sterck, F., Markesteijn, L., Schieving, F., Poorter, L., 2011. Functional traits determine trade-offs and niches in a tropical forest community. Proceedings of the National Academy of Sciences 108, 20627-20632. https://doi.org/10.1073/pnas.1106950108

Yang, Y.H., Wang, G., Xiong, Y.C., 2015. The contribution of intraspecific trait variability to plant community assembly patterns on the niche-neutral continuum. Pakistan Journal of Botany 47, 1039-1050.

Yenni, G., Adler, P.B., Ernest, S.K.M., 2012. Strong self-limitation promotes the persistence of rare species. Ecology 93, 456461. https://doi.org/10.1890/11-1087.1

Frenette-Dussault, C., Shipley, B., Meziane, D., Hingrat, Y., 2013. Trait-based climate change predictions of plant community structure in arid steppes. Journal of Ecology 101, 484-492. https://doi.org/10.1111/1365-2745.12040

Laliberté, E., Shipley, B., Norton, D.A., Scott, D., 2012. Which plant traits determine abundance under long-term shifts in soil resource availability and grazing intensity?: Plant traits and species abundance. Journal of Ecology 100, 662-677. https://doi.org/10.1111/j.1365-2745.2011.01947.x

Crowther, T.W., Maynard, D.S., Crowther, T.R., Peccia, J., Smith, J.R., Bradford, M.A., 2014. Untangling the fungal niche: the trait-based approach. Frontiers in Microbiology 5. https://doi.org/10.3389/fmicb.2014.00579

Colbach, N., Granger, S., Guyot, S.H.M., Mézière, D., 2014. A trait-based approach to explain weed species response to agricultural practices in a simulation study with a cropping system model. Agriculture, Ecosystems \& Environment 183, 197-204. https://doi.org/10.1016/j.agee.2013.11.013

Moor, H., 2017. Life history trade-off moderates model predictions of diversity loss from climate change. PLOS ONE 12, e0177778. https://doi.org/10.1371/journal.pone.0177778

Falster, D.S., Brännström, Å., Dieckmann, U., Westoby, M., 2011. Influence of four major plant traits on average height, leafarea cover, net primary productivity, and biomass density in single-species forests: A theoretical investigation. J. Ecol. 99, 148164. https://doi.org/10.1111/j.1365-2745.2010.01735.x

\section{Model type \\ statistical}

Target scale

local, regional,

community

individual-based

community

statistical

community

statistical

global

equation-based

community

equation-based

community

equation-based

community

statistical

community

statistical

community

conceptual

community

equation-based

species

equation-based

community

equation-based

landscape 


Section
1.4. Trait-based models of com-
munity dynamics
1.4. Trait-based models of com-
munity dynamics
1.4. Trait-based models of com-
munity dynamics
1.4. Trait-based models of com-
munity dynamics

1.4. Trait-based models of community dynamics

1.4. Trait-based models of community dynamics

1.4. Trait-based models of community dynamics

1.4. Trait-based models of community dynamics

1.4. Trait-based models of community dynamics

1.4. Trait-based models of community dynamics

1.5. Trait-based dynamic global vegetation models (DGVMs)

1.5. Trait-based dynamic global vegetation models (DGVMs)

1.5. Trait-based dynamic global vegetation models (DGVMs)

\section{Reference}

Jabot, F., 2010. A stochastic dispersal-limited trait-based model of community dynamics. J. Theor. Biol. 262, 650-661.

Quétier, F., Lavorel, S., Liancourt, P., Thébault, A., Davies, I.D., 2011. Assessing long-term land-use legacies in subalpine grasslands by using a plant trait-based generic modelling framework. Plant Ecology \& Diversity 4, 391-402. https://doi.org/10.1080/17550874.2011.629232

Savage, V.M., Webb, C.T., Norberg, J., 2007. A general multi-trait-based framework for studying the effects of biodiversity on ecosystem functioning. Journal of Theoretical Biology 247, 213-229. https://doi.org/10.1016/j.jtbi.2007.03.007

Seifan, M., Seifan, T., Jeltsch, F., Tielbörger, K., 2012. Combined disturbances and the role of their spatial and temporal properties in shaping community structure. Perspect. Plant Ecol. Evol. Syst. 14, 217-229.

https://doi.org/10.1016/j.ppees.2011.11.003

Seifan, M., Seifan, T., Schiffers, K., Jeltsch, F., Tielbörger, K., 2013. Beyond the Competition-Colonization Trade-Off: Linking Multiple Trait Response to Disturbance Characteristics. Am. Nat. 181, 151-160. https://doi.org/10.1086/668844

Tanaka, Y., 2012. Trait response in communities to environmental change: effect of interspecific competition and trait covariance structure. Theoretical Ecology 5, 83-98. https://doi.org/10.1007/s12080-010-0100-2

Weiss, L., Jeltsch, F., 2015. The response of simulated grassland communities to the cessation of grazing. Ecological Modelling 303, 1-11. https://doi.org/10.1016/j.ecolmodel.2015.02.002

Weiss, L., Pfestorf, H., May, F., Körner, K., Boch, S., Fischer, M., Müller, J., Prati, D., Socher, S.A., Jeltsch, F., 2014. Grazing response patterns indicate isolation of semi-natural European grasslands. Oikos 123, 599-612. https://doi.org/10.1111/j.16000706.2013.00957.x

Harper, A.B., Cox, P.M., Friedlingstein, P., Wiltshire, A.J., Jones, C.D., Sitch, S., Mercado, L.M., Groenendijk, M., Robertson, E., Kattge, J., Bönisch, G., Atkin, O.K., Bahn, M., Cornelissen, J., Niinemets, Ü., Onipchenko, V., Peñuelas, J., Poorter, L., Reich, P.B., Soudzilovskaia, N.A., Bodegom, P. van, 2016. Improved representation of plant functional types and physiology in the Joint UK Land Environment Simulator (JULES v4.2) using plant trait information. Geoscientific Model Development 9, 2415-2440. https://doi.org/10.5194/gmd-9-2415-2016

Holzwarth, F., Rüger, N., Wirth, C., 2015. Taking a closer look: disentangling effects of functional diversity on ecosystem functions with a trait-based model across hierarchy and time. R. Soc. open Sci. 2, 140541. https://doi.org/10.1098/rsos.140541

equation-based

ecosystem

equation-based

ecosystem

individual-based

community

individual-based

community

equation-based

community

individual-based

community

individual-based

community

Pavlick, R., Drewry, D.T., Bohn, K., Reu, B., Kleidon, a., 2012. The Jena Diversity-Dynamic Global Vegetation Model (JeDiDGVM): a diverse approach to representing terrestrial biogeography and biogeochemistry based on plant functional trade-offs. Biogeosciences Discuss. 9, 4627-4726. https://doi.org/10.5194/bgd-9-4627-2012 equation-based global 


\begin{tabular}{l}
\hline Section \\
\hline 1.5 . Trait-based dynamic global \\
vegetation models (DGVMs)
\end{tabular}

1.5. Trait-based dynamic global vegetation models (DGVMs)

1.5. Trait-based dynamic global vegetation models (DGVMs)

1.5. Trait-based dynamic global vegetation models (DGVMs)

1.5. Trait-based dynamic global vegetation models (DGVMs)

1.5. Trait-based dynamic global vegetation models (DGVMs)

1.5. Trait-based dynamic global vegetation models (DGVMs)

1.5. Trait-based dynamic global vegetation models (DGVMs)

1.5. Trait-based dynamic global vegetation models (DGVMs)

1.5. Trait-based dynamic global vegetation models (DGVMs)

1.6. Trait-based models of plant invasions

1.6. Trait-based models of plant invasions

1.6. Trait-based models of plant invasions

\section{Reference}

Reu, B., Proulx, R., Bohn, K., Dyke, J.G., Kleidon, A., Pavlick, R., Schmidtlein, S., 2010. The role of climate and plant functional trade-offs in shaping global biome and biodiversity patterns. Glob. Ecol. Biogeogr. 20, 570-581.

https://doi.org/10.1111/j.1466-8238.2010.00621.x

Reu, B., Zaehle, S., Proulx, R., Bohn, K., Kleidon, A., Pavlick, R., Schmidtlein, S., 2011. The role of plant functional trade-offs equation-based for biodiversity changes and biome shifts under scenarios of global climatic change. Biogeosciences 8, 1255-1266.

https://doi.org/10.5194/bg-8-1255-2011

Sakschewski, B., von Bloh, W., Boit, A., Rammig, A., Kattge, J., Poorter, L., Peñuelas, J., Thonicke, K., 2015. Leaf and stem economics spectra drive diversity of functional plant traits in a dynamic global vegetation model. Glob. Chang. Biol. 21, 27112725. https://doi.org/10.1111/gcb.12870

Scheiter, S., Higgins, S.I., 2009. Impacts of climate change on the vegetation of Africa: An adaptive dynamic vegetation modelling approach. Glob. Chang. Biol. 15, 2224-2246. https://doi.org/10.1111/j.1365-2486.2008.01838.x

Scheiter, S., Langan, L., Higgins, S.I., 2013. Next-generation dynamic global vegetation models: learning from community ecology. New Phytol. 198, 957-69. https://doi.org/10.1111/nph.12210

Smith, B., Prentice, I.C., Climate, M.T.S., Sykes, M.T., 2001. Representation of vegetation dynamics in the modelling of terrestrial ecosystems : comparing two contrasting approaches within European climate space. Glob. Ecol. Biogeogr. 10, 621-637. https://doi.org/10.1046/j.1466-822X.2001.00256.x

Van Bodegom, P.M., Douma, J.C., Witte, J.P.M.M., Ordoñez, J.C., Bartholomeus, R.P., Aerts, R., 2012. Going beyond limitations of plant functional types when predicting global ecosystem-atmosphere fluxes: exploring the merits of traits-based approaches. Glob. Ecol. Biogeogr. 21, 625-636. https://doi.org/10.1111/j.1466-8238.2011.00717.x

Verheijen, L.M., Brovkin, V., Aerts, R., Bönisch, G., Cornelissen, J.H.C., Kattge, J., Reich, P.B., Wright, I.J., Van Bodegom, P.M., 2013. Impacts of trait variation through observed trait-climate relationships on performance of an Earth system model: A conceptual analysis. Biogeosciences 10, 5497-5515. https://doi.org/10.5194/bg-10-5497-2013

Walker, A.P., Quaife, T., van Bodegom, P.M., De Kauwe, M.G., Keenan, T.F., Joiner, J., Lomas, M.R., MacBean, N., Xu, C. Yang, X., Woodward, F I., 2017. The impact of alternative trait-scaling hypotheses for the maximum photosynthetic carboxylation rate (Vcmax) on global gross primary production. New Phytol. 215, 1370-1386. https://doi.org/10.1111/nph.14623

Yang, Y., Zhu, Q., Peng, C., Wang, H., Xue, W., Lin, G., Wen, Z., Chang, J., Wang, M., Liu, G., Li, S., 2016. A novel approach for modelling vegetation distributions and analysing vegetation sensitivity through trait-climate relationships in China. Scientific Reports 6. https://doi.org/10.1038/srep24110

Herron, P.M., Martine, C.T., Latimer, A.M., Leicht-Young, S.A., 2007. Invasive plants and their ecological strategies: Prediction and explanation of woody plant invasion in New England. Divers. Distrib. 13, 633-644. https://doi.org/10.1111/j.14724642.2007.00381.x

Higgins, S.I., Richardson, D.M., 1998. Pine invasions in the southern hemisphere: modeling interactions between organism, enviroment and disturbance. Plant Ecol. 135, 79-93. https://doi.org/10.1023/a:1009760512895

Küster, E.C., Kühn, I., Bruelheide, H., Klotz, S., 2008. Trait interactions help explain plant invasion success in the German flora. J. Ecol. 96, 860-868. https://doi.org/10.1111/j.1365-2745.2008.01406.x equation-based + individual-based

global

equation-based + individual-based

global

equation-based + individual-basec

global

equation-based + individual-based

equation-based

equation-based

global

equation-based

global

equation-based

global

statistical

specie

individual-based

community

statistical

species 


\begin{tabular}{l}
\hline Section \\
1.6. Trait-based models of plant \\
invasions
\end{tabular}

1.6. Trait-based models of plant invasions

1.7. Trait-based models of ecosystem services

1.7. Trait-based models of ecosystem services

1.7. Trait-based models of ecosystem services

1.7. Trait-based models of ecosystem services

1.7. Trait-based models of ecosystem services

1.7. Trait-based models of ecosystem services

1.7. Trait-based models of ecosystem services

1.7. Trait-based models of ecosystem services

1.8. Trait-based models on interactions between plants and other organisms

1.8. Trait-based models on interactions between plants and other organisms

1.8. Trait-based models on interactions between plants and other organisms

\section{Reference}

Otfinowski, R., Kenkel, N.C., Dixon, P., Wilmshurst, J.F., 2007. Integrating climate and trait models to predict the invasivenes

statistical

species https://doi.org/10.4141/CJPS07117

Radny, J., Meyer, K.M., 2018. The role of biotic factors during plant establishment in novel communities assessed with an agent-based simulation model. PeerJ 6, e5342. https://doi.org/10.7717/peerj.5342

individual-based

community

Astegiano, J., Guimarães, P.R., Cheptou, P.-O., Vidal, M.M., Mandai, C.Y., Ashworth, L., Massol, F., 2015. Persistence of Plants and Pollinators in the Face of Habitat Loss, in: Advances in Ecological Research. Elsevier, pp. 201-257.

Grigulis, K., Lavorel, S., Krainer, U., Legay, N., Baxendale, C., Dumont, M., Kastl, E., Arnoldi, C., Bardgett, R.D., Poly, F. Pommier, T., Schloter, M., Tappeiner, U., Bahn, M., Clément, J.-C., 2013. Relative contributions of plant traits and soil microbial properties to mountain grassland ecosystem services. J. Ecol. 101, 47-57. https://doi.org/10.1111/1365-2745.12014

Homolova, L., Schaepman, M.E., Lamarque, P., Clevers, J.G.P.W., De Bello, F., Thuiller, W., Lavorel, S., 2014. Comparison of remote sensing and plant trait-based modelling to predict ecosystem services in subalpine grasslands. Ecosphere 5, 1-29. https://doi.org/10.1890/ES13-00393.1

Lamarque, P., Lavorel, S., Mouchet, M., Quétier, F., 2014. Plant trait-based models identify direct and indirect effects of climate change on bundles of grassland ecosystem services. Proc. Natl. Acad. Sci. U. S. A. 111, 13751-6. https://doi.org/10.1073/pnas.1216051111

Lavorel, S., Grigulis, K., Lamarque, P., Colace, M.P., Garden, D., Girel, J., Pellet, G., Douzet, R., 2011. Using plant functional traits to understand the landscape distribution of multiple ecosystem services. J. Ecol. 99, 135-147.

https://doi.org/10.1111/j.1365-2745.2010.01753.x

Lochon, I., Colace, M.-P., Devaux, C., Grigulis, K., Rettinger, R., Lavorel, S., 2018. Taxonomic and functional facets of the resilience to management of mown subalpine grasslands. Applied Vegetation Science 21, 636-646. https://doi.org/10.1111/avsc.12392

Quétier, F., Lavorel, S., Thuiller, W., Davies, I., 2007. PLANT-TRAIT-BASED MODELING ASSESSMENT OF ECOSYSTEM-SERVICE SENSITIVITY TO LAND-USE CHANGE. Ecological Applications 17, 2377-2386. https://doi.org/10.1890/06-0750.1

Schirpke, U., Kohler, M., Leitinger, G., Fontana, V., Tasser, E., Tappeiner, U., 2017. Future impacts of changing land-use and climate on ecosystem services of mountain grassland their resilience. Ecosyst. Serv. 26, 79-94 https://doi.org/10.1016/j.ecoser.2017.06.008

Zhu, Q., Iversen, C.M., Riley, W.J., Slette, I.J., Vander Stel, H.M., 2016. Root traits explain observed tundra vegetation nitrogen uptake patterns: Implications for trait-based land models: Tundra N Uptake Model-Data Comparison. Journal of Geophysical Research: Biogeosciences 121,3101-3112. https://doi.org/10.1002/2016JG003554

Lavorel, S., Storkey, J., Bardgett, R.D., De Bello, F., Berg, M.P., Le Roux, X., Moretti, M., Mulder, C., Pakeman, R.J., Díaz, S., Harrington, R., 2013. A novel framework for linking functional diversity of plants with other trophic levels for the quantification of ecosystem services. J. Veg. Sci. 24, 942-948. https://doi.org/10.1111/jvs.12083

Mortensen, B., Abbott, K.C., Danielson, B., 2018. Defensive tradeoffs are not prerequisites to plant diversity in a two species model. Oikos 127, 63-72. https://doi.org/10.1111/oik.04300 metacommunity

statistical

ecosystem

statistical

landscape, ecosystem

ecosystem

statistical

statistical

landscape, eco-

system

equation-based +

statistical

ecosystem

equation-based

ecosystem

statistical

ecosystem

equation-based

global

conceptual + statistical

equation-based

community 


\section{Section}

1.8. Trait-based models on interactions between plants and other organisms

1.8. Trait-based models on interactions between plants and other organisms

1.8. Trait-based models on interactions between plants and other organisms

1.8. Trait-based models on interactions between plants and other organisms

2. Trait-based modelling of animals in terrestrial ecosystems

2. Trait-based modelling of animals in terrestrial ecosystems

2. Trait-based modelling of animals in terrestrial ecosystems

2. Trait-based modelling of animals in terrestrial ecosystem

2. Trait-based modelling of animals in terrestrial ecosystem

2. Trait-based modelling of animals in terrestrial ecosystems

2. Trait-based modelling of animals in terrestrial ecosystems

2. Trait-based modelling of animals in terrestrial ecosystems

2. Trait-based modelling of animals in terrestrial ecosystems

\section{Reference}

Pöyry, J., Carvalheiro, L.G., Heikkinen, R.K., Kühn, I., Kuussaari, M., Schweiger, O., Valtonen, A., van Bodegom, P.M ,

Franzén, M., 2017. The effects of soil eutrophication propagate to higher trophic levels. Glob. Ecol. Biogeogr. 26, 18-30.

community

https://doi.org/10.1111/geb.12521

(c) Sontent-animal interactions. Ecography (Cop.). 38, 380-392.

https://doi.org/10.1111/ecog.00983

Ke, P.-J., Miki, T., Ding, T.-S., 2015. The soil microbial community predicts the importance of plant traits in plant-soil feedback. New Phytologist 206, 329-341. https://doi.org/10.1111/nph.13215

Jack, C.N., Friesen, M.L., Hintze, A., Sheneman, L., 2017. Third-party mutualists have contrasting effects on host invasion under the enemy-release and biotic-resistance hypotheses. Evolutionary Ecology 31, 829-845. https://doi.org/10.1007/s10682017-9912-5

Brousseau, Pierre-Marc Gravel, D., Handa, I.T., 2018. Trait-matching and phylogeny as predictors of predator-prey interaction involving ground beetles. Funct. Ecol. 12, 3218-3221. https://doi.org/10.1111/ijlh.12426

Buchmann, C.M., Schurr, F.M., Nathan, R., Jeltsch, F., 2011. An allometric model of home range formation explains the structuring of animal communities exploiting heterogeneous resources. Oikos 120, 106-118. https://doi.org/10.1111/j.1600-

0706.2010.18556.x

Gervasi, S.S., Stephens, P.R., Hua, J., Searle, C.L., Xie, G.Y., Urbina, J., Olson, D.H., Bancroft, B.A., Weis, V., Hammond,

J.I., Relyea, R.A., Blaustein, A.R., 2017. Linking ecology and epidemiology to understand predictors of multi-host responses to an emerging pathogen, the amphibian chytrid fungus. PLoS One 12,1-23. https://doi.org/10.1371/journal.pone.0167882

Hirt, M.R., Grimm, V., Li, Y., Rall, B.C., Rosenbaum, B., Brose, U., 2018. Bridging Scales: Allometric Random Walks Link Movement and Biodiversity Research. Trends in Ecology \& Evolution 33, 701-712. https://doi.org/10.1016/j.tree.2018.07.003

Hui, C. Richardson, D.M., Landi, P., Minoarivelo, H.O, Garnas, J., Roy, H.E 2016. Defining invasiveness and invasibility in ecological networks. Biological Invasions 18, 971-983. https://doi.org/10.1007/s10530-016-1076-7

Kearney, M., Phillips, B.L., Tracy, C.R., Christian, K.A., Betts, G., Porter, W.P., 2008. Modelling species distributions without using species distributions: the cane toad in Australia under current and future climates. Ecography (Cop.). 31, 423-434. https://doi.org/10.1111/j.2008.0906-7590-05457.x

Kolbe, J.J., Kearney, M., Shine, R., 2010. Modeling the consequences of thermal trait variation for the cane toad invasion of Australia. Ecol. Appl. 20, 2273-2285. https://doi.org/10.1890/09-1973.1

Lentini, P.E., Bird, T.J., Griffiths, S.R., Godinho, L.N., Wintle, B.A., 2015. A global synthesis of survival estimates for microbats. Biol. Lett. 11. https://doi.org/10.1098/rsbl.2015.0371

Ozgul, A., Coulson, T., Reynolds, A., Cameron, T.C., Benton, T.G., 2012. Population Responses to Perturbations: The Importance of Trait-Based Analysis Illustrated through a Microcosm Experiment. The American Naturalist 179, 582-594. https://doi.org/10.1086/664999 conceptual

community

equation-based

community

equation-based + individual-based

species

statistical

community

individual-base

individual, landscape

statistical

individual, species

individual-based

community

equation-based

community

statistical

statistical

scape

species, landscape

species

equation-based

population 


\begin{tabular}{ll}
\hline Section & Reference \\
\hline $\begin{array}{l}\text { 2. Trait-based modelling of ani- } \\
\text { mals in terrestrial ecosystems }\end{array}$ & $\begin{array}{l}\text { Pontarp, M., Wiens, J.J., 2017. The origin of species richness patterns along environmental gradients: uniting explanations } \\
\text { based on time, diversification rate and carrying capacity. J. Biogeogr. 44, 722-735. https://doi.org/10.1111/jbi.12896 }\end{array}$
\end{tabular}

mals in terrestrial ecosystems

based on time, diversification rate and carrying capacity. J. Biogeogr. 44, 722-735. https://doi.org/10.1111/jbi.12896

2. Trait-based modelling of animals in terrestrial ecosystems

2. Trait-based modelling of animals in terrestrial ecosystems

2. Trait-based modelling of animals in terrestrial ecosystems

2. Trait-based modelling of animals in terrestrial ecosystems

2. Trait-based modelling of animals in terrestrial ecosystems

2. Trait-based modelling of animals in terrestrial ecosystems

2. Trait-based modelling of animals in terrestrial ecosystems

2. Trait-based modelling of animals in terrestrial ecosystems

2. Trait-based modelling of animals in terrestrial ecosystems

2. Trait-based modelling of animals in terrestrial ecosystems

2. Trait-based modelling of animals in terrestrial ecosystems

2. Trait-based modelling of animals in terrestrial ecosystems
Porter, W.P., Kearney, M., 2009. Size, shape, and the thermal niche of endotherms. Proc. Natl. Acad. Sci. 106, 19666-19672. https://doi.org/10.1073/pnas.0907321106

Santini, L., Cornulier, T., Bullock, J.M., Palmer, S.C.F., White, S.M., Hodgson, J.A., Bocedi, G., Travis, J.M.J., 2016. A traitbased approach for predicting species responses to environmental change from sparse data: how well might terrestrial mammals track climate change? Global Change Biology 22, 2415-2424. https://doi.org/10.1111/gcb.13271

Scherer, C., Jeltsch, F., Grimm, V., Blaum, N., 2016. Merging trait-based and individual-based modelling: An animal functional type approach to explore the responses of birds to climatic and land use changes in semi-arid African savannas. Ecol. Modell. 326, 75-89. https://doi.org/10.1016/j.ecolmodel.2015.07.005

Schlossberg, S., Chase, M.J., Griffin, C.R., 2018. Using species traits to predict detectability of animals on aerial surveys: Ecol. statistical Appl. https://doi.org/10.1002/eap.1632

Smallegange, I.M., Ens, H.M., 2018. Trait-based predictions and responses from laboratory mite populations to harvesting in stochastic environments. J. Anim. Ecol. 87, 893-905. https://doi.org/10.1111/1365-2656.12802

van Benthem, K.J., Froy, H., Coulson, T., Getz, L.L., Oli, M.K., Ozgul, A., 2017. Trait-demography relationships underlying small mammal population fluctuations. Journal of Animal Ecology 86, 348-358. https://doi.org/10.1111/1365-2656.12627

Pontarp, M., Petchey, O.L., 2016. Community trait overdispersion due to trophic interactions: concerns for assembly process inference. Proceedings of the Royal Society B: Biological Sciences 283, 20161729. https://doi.org/10.1098/rspb.2016.1729

Tenan, S., Brambilla, M., Pedrini, P. Sutherland, C. 2017. Quantifying spatial variation in the size and structure of ecologically stratified communities. Methods in Ecology and Evolution 8, 976-984. https://doi.org/10.1111/2041-210X.12719

Laubmeier, A.N., Wootton, K., Banks, J.E., Bommarco, R., Curtsdotter, A., Jonsson, T., Roslin, T., Banks, H.T., 2018. From theory to experimental design-Quantifying a trait-based theory of predator-prey dynamics. PLOS ONE 13, e0195919. https://doi.org/10.1371/journal.pone.0195919

Diamond, S.E., Nichols, L.M., McCoy, N., Hirsch, C., Pelini, S.L., Sanders, N.J., Ellison, A.M., Gotelli, N.J., Dunn, R.R., 2012. A physiological trait-based approach to predicting the responses of species to experimental climate warming. Ecology 93 2305-2312. https://doi.org/10.1890/11-2296.1

Hof, A.R., Rodríguez-Castañeda, G., Allen, A.M., Jansson, R., Nilsson, C., 2017. Vulnerability of Subarctic and Arctic breeding birds. Ecological Applications 27, 219-234. https://doi.org/10.1002/eap.1434

Haney, S.D., Siepielski, A.M., 2018. Tipping Points in Resource Abundance Drive Irreversible Changes in Community Structure. The American Naturalist 191, 668-675. https://doi.org/10.1086/697045 species, population 


Section
2. Trait-based modelling of ani-

Reference

mals in terrestrial ecosystems

3. Trait-based modelling of aquatic ecosystems

3. Trait-based modelling of aquatic ecosystems

3. Trait-based modelling of aquatic ecosystems

3. Trait-based modelling of aquatic ecosystems

3. Trait-based modelling of aquatic ecosystems

3. Trait-based modelling of aquatic ecosystems

3. Trait-based modelling of aquatic ecosystems

3. Trait-based modelling of aquatic ecosystems

3. Trait-based modelling of aquatic ecosystems

3.1. Trait-based models including fish

3.1. Trait-based models including fish

3.1. Trait-based models including fish
Ozgul, A., Bateman, A.W., English, S., Coulson, T., Clutton-Brock, T.H., 2014. Linking body mass and group dynamics in an obligate cooperative breeder. Journal of Animal Ecology 83, 1357-1366. https://doi.org/10.1111/1365-2656.12239

Madin, J.S., Baird, A.H., Dornelas, M., Connolly, S.R., 2014. Mechanical vulnerability explains size-dependent mortality of reef corals. Ecology Letters 17, 1008-1015. https://doi.org/10.1111/ele.12306

Alexandridis, N., Dambacher, J.M., Jean, F., Desroy, N., Bacher, C., 2017. Qualitative modelling of functional relationships in marine benthic communities. Ecological Modelling 360, 300-312. https://doi.org/10.1016/j.ecolmodel.2017.07.021

Castellani, M., Våge, S., Strand, E., Thingstad, T.F., Giske, J., 2013. The Scaled Subspaces Method: A new trait-based approach to model communities of populations with largely inhomogeneous density. Ecological Modelling 251, 173-186. https://doi.org/10.1016/j.ecolmodel.2012.12.006

Edmunds, P.J., Adjeroud, M., Baskett, M.L., Baums, I.B., Budd, A.F., Carpenter, R.C., Fabina, N.S., Fan, T.-Y., Franklin, E.C., Gross, K., Han, X., Jacobson, L., Klaus, J.S., McClanahan, T.R., O'Leary, J.K., van Oppen, M.J.H., Pochon, X., Putnam, H.M., Smith, T.B., Stat, M., Sweatman, H., van Woesik, R., Gates, R.D., 2014. Persistence and Change in Community Composition of Reef Corals through Present, Past, and Future Climates. PLoS ONE 9, e107525.

https://doi.org/10.1371/journal.pone.0107525

Gardner, R.H., Engelhardt, K.A.M., Elmore, A.J., Cadol, D., 2014. A traits-based model of species diversity. Ecol. Modell. 288, 178-194. https://doi.org/10.1016/j.ecolmodel.2014.06.006

Hagerthey, S.E., Newman, S., Xue, S., 2012. Periphyton-based transfer functions to assess ecological imbalance and management of a subtropical ombrotrophic peatland: Periphyton transfer functions. Freshwater Biology 57, 1947-1965.

https://doi.org/10.1111/j.1365-2427.2012.02848.x

Hug Peter, D., Sardy, S., Diaz Rodriguez, J., Castella, E., Slaveykova, V.I., 2018. Modeling whole body trace metal concentrations in aquatic invertebrate communities: A trait-based approach. Environ. Pollut. 233, 419-428.

https://doi.org/10.1016/j.envpol.2017.10.044

Montalto, V., Rinaldi, A., Sarà, G., 2015. Life history traits to predict biogeographic species distributions in bivalves. Sci. Nat. 102. https://doi.org/10.1007/s00114-015-1313-4

Rinaldi, A., Montalto, V., Manganaro, A., Mazzola, A., Mirto, S., Sanfilippo, M., Sarà, G., Sara, G., 2014. Predictive mechanistic bioenergetics to model habitat suitability of shellfish culture in coastal lakes. Estuar. Coast. Shelf Sci. 144, 89-98. https://doi.org/10.1016/j.ecss.2014.04.013

Huebert, K.B., Pätsch, J., Hufnagl, M., Kreus, M., Peck, M.A., 2018. Modeled larval fish prey fields and growth rates help predict recruitment success of cod and anchovy in the North Sea. Mar. Ecol. Prog. Ser. 600, 111-126. https://doi.org/10.3354/meps12615

Andersen, K.H., Beyer, J.E., 2006. Asymptotic Size Determines Species Abundance in the Marine Size Spectrum. Am. Nat. 168, 54-61. https://doi.org/10.1086/504849

Andersen, K.H., Brander, K., Ravn-Jonsen, L., 2015. Trade-offs between objectives for ecosystem management of fisheries. Ecol. Appl. 25, 1390-1396. https://doi.org/10.1890/14-1209.1 


\begin{tabular}{|c|c|}
\hline Section & Reference \\
\hline $\begin{array}{l}\text { 3.1. Trait-based models includ- } \\
\text { ing fish }\end{array}$ & $\begin{array}{l}\text { Andersen, K.H., Pedersen, M., 2010. Damped trophic cascades driven by fishing in model marine ecosystems. Proc. R. Soc. B } \\
\text { Biol. Sci. 277, 795-802. https://doi.org/10.1098/rspb.2009.1512 }\end{array}$ \\
\hline $\begin{array}{l}\text { 3.1. Trait-based models includ- } \\
\text { ing fish }\end{array}$ & $\begin{array}{l}\text { Winemiller, K.O., Rose, K.A., 1992. Patterns of life-history in North American: Implications for Population Regulation. Can. J. } \\
\text { Fish. Aquat. Sci. 49, 2196-2218. https://doi.org/10.1139/f92-242 }\end{array}$ \\
\hline $\begin{array}{l}\text { 3.1. Trait-based models includ- } \\
\text { ing fish }\end{array}$ & $\begin{array}{l}\text { Andersen, K.H., Rice, J.C., 2010. Direct and indirect community effects of rebuilding plans. ICES J. Mar. Sci. 67, 1980-1988. } \\
\text { https://doi.org/10.1093/icesjms/fsq035 }\end{array}$ \\
\hline $\begin{array}{l}\text { 3.1. Trait-based models includ- } \\
\text { ing fish }\end{array}$ & $\begin{array}{l}\text { Bennett, M.G., Whiles, M.R., Whitledge, G.W., 2016. Population-level responses of life history traits to flow regime in three } \\
\text { common stream fish species: Fish Intraspecific Flow-Life History Relationships. Ecohydrology 9, 1388-1399. } \\
\text { https://doi.org/10.1002/eco.1734 }\end{array}$ \\
\hline $\begin{array}{l}\text { 3.1. Trait-based models includ- } \\
\text { ing fish }\end{array}$ & $\begin{array}{l}\text { Brochier, T., Ecoutin, J.M., de Morais, L.T., Kaplan, D.M., Lae, R., 2013. A multi-agent ecosystem model for studying chang- } \\
\text { es in a tropical estuarine fish assemblage within a marine protected area. Aquat. Living Resour. 26, 147-158. } \\
\text { https://doi.org/10.1051/alr/2012028 }\end{array}$ \\
\hline $\begin{array}{l}\text { 3.1. Trait-based models includ- } \\
\text { ing fish }\end{array}$ & $\begin{array}{l}\text { Castellani, M., Rosland, R., Urtizberea, A., Fiksen, Ø., 2013. A mass-balanced pelagic ecosystem model with size-structured } \\
\text { behaviourally adaptive zooplankton and fish. Ecol. Modell. 251, 54-63. https://doi.org/10.1016/j.ecolmodel.2012.12.007 }\end{array}$ \\
\hline $\begin{array}{l}\text { 3.1. Trait-based models includ- } \\
\text { ing fish }\end{array}$ & $\begin{array}{l}\text { Guiet, J., Aumont, O., Poggiale, J.-C., Maury, O., 2016. Effects of lower trophic level biomass and water temperature on fish } \\
\text { communities: A modelling study. Progress in Oceanography 146, 22-37. https://doi.org/10.1016/j.pocean.2016.04.003 }\end{array}$ \\
\hline $\begin{array}{l}\text { 3.1. Trait-based models includ- } \\
\text { ing fish }\end{array}$ & $\begin{array}{l}\text { Guiet, J., Poggiale, J.C., Maury, O., 2016. Modelling the community size-spectrum: recent developments and new directions. } \\
\text { Ecol. Modell. 337, 4-14. https://doi.org/10.1016/j.ecolmodel.2016.05.015 }\end{array}$ \\
\hline $\begin{array}{l}\text { 3.1. Trait-based models includ- } \\
\text { ing fish }\end{array}$ & $\begin{array}{l}\text { Hartvig, M., Andersen, K.H., 2013. Coexistence of structured populations with size-based prey selection. Theor. Popul. Biol. } \\
\text { 89, 24-33. https://doi.org/10.1016/j.tpb.2013.07.003 }\end{array}$ \\
\hline $\begin{array}{l}\text { 3.1. Trait-based models includ- } \\
\text { ing fish }\end{array}$ & $\begin{array}{l}\text { Hartvig, M., Andersen, K.H., Beyer, J.E., 2011. Food web framework for size-structured populations. J. Theor. Biol. 272, 113- } \\
\text { 122. https://doi.org/10.1016/j.jtbi.2010.12.006 }\end{array}$ \\
\hline $\begin{array}{l}\text { 3.1. Trait-based models includ- } \\
\text { ing fish }\end{array}$ & $\begin{array}{l}\text { Houle, J.E., Andersen, K.H., Farnsworth, K.D., Reid, D.G., 2013. Emerging asymmetric interactions between forage and preda- } \\
\text { tor fisheries impose management trade-offs: forage and predator fishery interactions. Journal of Fish Biology n/a-n/a. } \\
\text { https://doi.org/10.1111/jfb.12163 }\end{array}$ \\
\hline $\begin{array}{l}\text { 3.1. Trait-based models includ- } \\
\text { ing fish }\end{array}$ & $\begin{array}{l}\text { Houle, J.E., de Castro, F., Cronin, M.A., Farnsworth, K.D., Gosch, M., Reid, D.G., 2016. Effects of seal predation on a mod- } \\
\text { elled marine fish community and consequences for a commercial fishery. Journal of Applied Ecology 53, 54-63. } \\
\text { https://doi.org/10.1111/1365-2664.12548 }\end{array}$ \\
\hline $\begin{array}{l}\text { 3.1. Trait-based models includ- } \\
\text { ing fish }\end{array}$ & $\begin{array}{l}\text { Howeth, J.G., Gantz, C.A., Angermeier, P.L., Frimpong, E.A., Hoff, M.H., Keller, R.P., Mandrak, N.E., Marchetti, M.P., Old- } \\
\text { en, J.D., Romagosa, C.M., Lodge, D.M., 2016. Predicting invasiveness of species in trade: climate match, trophic guild and } \\
\text { fecundity influence establishment and impact of non-native freshwater fishes. Divers. Distrib. } 22,148-160 . \\
\text { https://doi.org/10.1111/ddi.12391 }\end{array}$ \\
\hline
\end{tabular}




\begin{tabular}{|c|c|}
\hline Section & Reference \\
\hline $\begin{array}{l}\text { 3.1. Trait-based models includ- } \\
\text { ing fish }\end{array}$ & $\begin{array}{l}\text { Jacobsen, N.S., Gislason, H., Andersen, K.H., 2014. The consequences of balanced harvesting of fish communities. Proc. R. } \\
\text { Soc. B Biol. Sci. 281, 20132701-20132701. https://doi.org/10.1098/rspb.2013.2701 }\end{array}$ \\
\hline $\begin{array}{l}\text { 3.1. Trait-based models includ- } \\
\text { ing fish }\end{array}$ & $\begin{array}{l}\text { Jennings, S., Collingridge, K., 2015. Predicting consumer biomass, size-structure, production, catch potential, responses to } \\
\text { fishing and associated uncertainties in the world's marine ecosystems. PLoS One 10,1-28. } \\
\text { https://doi.org/10.1371/journal.pone. } 0133794\end{array}$ \\
\hline $\begin{array}{l}\text { 3.1. Trait-based models includ- } \\
\text { ing fish }\end{array}$ & $\begin{array}{l}\text { Kolding, J., Jacobsen, N.S., Andersen, K.H., van Zwieten, P.A.M., 2016. Maximizing fisheries yields while maintaining com- } \\
\text { munity structure. Can. J. Fish. Aquat. Sci. 73, 644-655. https://doi.org/10.1139/cjfas-2015-0098 }\end{array}$ \\
\hline $\begin{array}{l}\text { 3.1. Trait-based models includ- } \\
\text { ing fish }\end{array}$ & $\begin{array}{l}\text { Maury, O., Poggiale, J.-C.C., 2013. From individuals to populations to communities: A dynamic energy budget model of ma- } \\
\text { rine ecosystem size-spectrum including life history diversity. J. Theor. Biol. 324, 52-71. } \\
\text { https://doi.org/10.1016/j.jtbi.2013.01.018 }\end{array}$ \\
\hline $\begin{array}{l}\text { 3.1. Trait-based models includ- } \\
\text { ing fish }\end{array}$ & $\begin{array}{l}\text { O’Farrell, S., Salguero-Gómez, R., van Rooij, J.M., Mumby, P.J., 2015. Disentangling trait-based mortality in species with } \\
\text { decoupled size and age. Journal of Animal Ecology 84, 1446-1456. https://doi.org/10.1111/1365-2656.12399 }\end{array}$ \\
\hline $\begin{array}{l}\text { 3.1. Trait-based models includ- } \\
\text { ing fish }\end{array}$ & $\begin{array}{l}\text { Schnedler-Meyer, N.A., Mariani, P., Kiørboe, T., 2016. The global susceptibility of coastal forage fish to competition by large } \\
\text { jellyfish. Proc. R. Soc. B Biol. Sci. 283. https://doi.org/10.1098/rspb.2016.1931 }\end{array}$ \\
\hline $\begin{array}{l}\text { 3.1. Trait-based models includ- } \\
\text { ing fish }\end{array}$ & $\begin{array}{l}\text { Shea, C.P., Bettoli, P.W., Potoka, K.M., Saylor, C.F., Shute, P.W., 2015. Use of Dynamic Occupancy Models to Assess the } \\
\text { Response of Darters (Teleostei: Percidae) to Varying Hydrothermal Conditions in a Southeastern United States Tailwater: } \\
\text { DARTER PATCH DYNAMICS. River Research and Applications 31, 676-691. https://doi.org/10.1002/rra.2766 }\end{array}$ \\
\hline $\begin{array}{l}\text { 3.1. Trait-based models includ- } \\
\text { ing fish }\end{array}$ & $\begin{array}{l}\text { Vindenes, Y., Edeline, E., Ohlberger, J., Langangen, Ø., Winfield, I.J., Stenseth, N.C., Vøllestad, L.A., 2014. Effects of Cli- } \\
\text { mate Change on Trait-Based Dynamics of a Top Predator in Freshwater Ecosystems. The American Naturalist 183, } 243-256 . \\
\text { https://doi.org/10.1086/674610 }\end{array}$ \\
\hline $\begin{array}{l}\text { 3.1. Trait-based models includ- } \\
\text { ing fish }\end{array}$ & $\begin{array}{l}\text { Zhang, L., Thygesen, U.H., Knudsen, K., Andersen, K.H., 2013. Trait diversity promotes stability of community dynamics. } \\
\text { Theor. Ecol. 6, 57-69. https://doi.org/10.1007/s12080-012-0160-6 }\end{array}$ \\
\hline $\begin{array}{l}\text { 3.1. Trait-based models includ- } \\
\text { ing fish }\end{array}$ & $\begin{array}{l}\text { Zhang, L., Hartvig, M., Knudsen, K., Andersen, K.H., 2014. Size-based predictions of food web patterns. Theoretical Ecology } \\
\text { 7, 23-33. https://doi.org/10.1007/s12080-013-0193-5 }\end{array}$ \\
\hline $\begin{array}{l}\text { 3.1. Trait-based models includ- } \\
\text { ing fish }\end{array}$ & $\begin{array}{l}\text { Zhang, L., Takahashi, D., Hartvig, M., Andersen, K.H., 2017. Food-web dynamics under climate change. Proceedings of the } \\
\text { Royal Society B: Biological Sciences 284, 20171772. https://doi.org/10.1098/rspb.2017.1772 }\end{array}$ \\
\hline $\begin{array}{l}\text { 3.1. Trait-based models includ- } \\
\text { ing fish }\end{array}$ & $\begin{array}{l}\text { Melián, C.J., Baldó, F., Matthews, B., Vilas, C., González-Ortegón, E., Drake, P., Williams, R.J., 2014. Individual Trait Varia- } \\
\text { tion and Diversity in Food Webs, in: Advances in Ecological Research. Elsevier, pp. 207-241. }\end{array}$ \\
\hline $\begin{array}{l}\text { 3.2. Trait-based models focusing } \\
\text { on plankton }\end{array}$ & $\begin{array}{l}\text { Acevedo-Trejos, E., Brandt, G., Bruggeman, J., Merico, A., 2015. Mechanisms shaping size structure and functional diversity } \\
\text { of phytoplankton communities in the ocean. Sci. Rep. 5, 17-20. https://doi.org/10.1038/srep08918 }\end{array}$ \\
\hline
\end{tabular}


3.2. Trait-based models focusing on plankton

Beckett, S.J., Weitz, J.S., 2018. The Effect of Strain Level Diversity on Robust Inference of Virus-Induced Mortality of Phytoplankton. Front. Microbiol. 9, 1-15. https://doi.org/10.3389/fmicb.2018.01850

3.2. Trait-based models focusing on plankton

3.2. Trait-based models focusing on plankton

3.2. Trait-based models focusing on plankton

3.2. Trait-based models focusing on plankton

3.2. Trait-based models focusing on plankton

3.2. Trait-based models focusing on plankton

3.2. Trait-based models focusing on plankton

3.2. Trait-based models focusing on plankton

3.2. Trait-based models focusing on plankton

3.2. Trait-based models focusing on plankton

3.2. Trait-based models focusing on plankton

Berge, T., Chakraborty, S., Hansen, P.J., Andersen, K.H., 2017. Modeling succession of key resource-harvesting traits of mixotrophic plankton. ISME J. 11, 212-223. https://doi.org/10.1038/ismej.2016.92

Bestion, E., García-Carreras, B., Schaum, C.-E., Pawar, S., Yvon-Durocher, G., 2018. Metabolic traits predict the effects of warming on phytoplankton competition. Ecology Letters 21, 655-664. https://doi.org/10.1111/ele.12932

Bruggeman, J., Kooijman, S.A.L.M., 2007. A biodiversity-inspired approach to aquatic ecosystem modeling. Limnol. Oceanogr. 52, 1533-1544. https://doi.org/10.4319/lo.2007.52.4.1533

Chen, B., Smith, S.L., 2018. CITRATE 1.0: Phytoplankton continuous trait-distribution model with one-dimensional physica transport applied to the Northwest Pacific. Geosci. Model Dev. 11, 467-495. https://doi.org/10.5194/gmd-11-467-2018

Clark, J.R., Lenton, T.M., Williams, H.T.P., Daines, S.J., 2013. Environmental selection and resource allocation determine spatial patterns in picophytoplankton cell size. Limnol. Oceanogr. 58, 1008-1022. https://doi.org/10.4319/lo.2013.58.3.1008

Cuesta, J.A., Delius, G.W., Law, R., 2018. Sheldon spectrum and the plankton paradox: two sides of the same coin-a traitbased plankton size-spectrum model. Journal of Mathematical Biology 76, 67-96. https://doi.org/10.1007/s00285-017-1132-7

Follows, M.J., Dutkiewicz, S., Grant, S., Chisholm, S.W., 2007. Emergent biogeography of microbial communities in a model ocean. Science. 315, 1843-1846. https://doi.org/10.1126/science.1138544

Gaedke, U., Klauschies, T., 2017. Analyzing the shape of observed trait distributions enables a data-based moment closure of aggregate models: Trait distributions and aggregate models. Limnology and Oceanography: Methods 15, 979-994. https://doi.org/10.1002/lom3.10218

Glibert, P.M., 2016. Margalef revisited: A new phytoplankton mandala incorporating twelve dimensions, including nutritiona physiology. Harmful Algae 55, 25-30. https://doi.org/10.1016/j.hal.2016.01.008

Hickman, A., Dutkiewicz, S., Williams, R., Follows, M., 2010. Modelling the effects of chromatic adaptation on phytoplankton community structure in the oligotrophic ocean. Marine Ecology Progress Series 406, 1-17. https://doi.org/10.3354/meps08588

Kiørboe, T., 2011. How zooplankton feed: Mechanisms, traits and trade-offs. Biol. Rev. 86, 311-339.

https://doi.org/10.1111/j.1469-185X.2010.00148.x 


Section
3.2. Trait-based models focusing
on plankton
3.2. Trait-based models focusing
on plankton
3.2. Trait-based models focusing
on plankton
3.2. Trait-based models focusing
on plankton
3.2. Trait-based models focusing
on plankton
3.2. Trait-based models focusing
on plankton
3.2. Trait-based models focusing
on plankton

3.2. Trait-based models focusing on plankton

3.2. Trait-based models focusing on plankton

3.2. Trait-based models focusing on plankton

4. Trait-based models on microorganisms and soil decomposers

\section{Reference}

Litchman, E., Klausmeier, C.A., Schofield, O.M., Falkowski, P.G., 2007. The role of functional traits and trade-offs in structur-

ing phytoplankton communities: Scaling from cellular to ecosystem level. Ecol. Lett. 10, 1170-1181.

https://doi.org/10.1111/j.1461-0248.2007.01117.x

ro-invertebrates in Danish waters: An agent-based approach. Reg. Stud. Mar. Sci. 20, 45-59.

https://doi.org/10.1016/J.RSMA.2018.03.005

Peeters, F., Straile, D., 2018. Trait selection and co-existence of phytoplankton in partially mixed systems: Trait based modelling and potential of an aggregated approach. PLOS ONE 13, e0194076. https://doi.org/10.1371/journal.pone.0194076

Shocket, M.S., Strauss, A.T., Hite, J.L., Šljivar, M., Civitello, D.J., Duffy, M.A., Cáceres, C.E., Hall, S.R., 2018. Temperature Drives Epidemics in a Zooplankton-Fungus Disease System: A Trait-Driven Approach Points to Transmission via Host Foraging. The American Naturalist 191, 435-451. https://doi.org/10.1086/696096

Roelke, D.L., Spatharis, S., 2015. Phytoplankton succession in recurrently fluctuating environments. PLoS One 10, 1-17. https://doi.org/10.1371/journal.pone.0121392

Roelke, D.L., Spatharis, S., 2015b. Phytoplankton assemblage characteristics in recurrently fluctuating environments. PLoS One 10, 1-25. https://doi.org/10.1371/journal.pone.0120673

Smeti, E., Roelke, D.L., Tsirtsis, G., Spatharis, S., 2018. Species extinctions strengthen the relationship between biodiversity and resource use efficiency. Ecological Modelling 384, 75-86. https://doi.org/10.1016/j.ecolmodel.2018.06.006

Smith, S., Merico, A., Hohn, S., Brandt, G., 2014. Sizing-up nutrient uptake kinetics: combining a physiological trade-off with size-scaling of phytoplankton traits. Marine Ecology Progress Series 511, 33-39. https://doi.org/10.3354/meps10903

Strauss, A.T., Civitello, D.J., Cáceres, C.E., Hall, S.R., 2015. Success, failure and ambiguity of the dilution effect among competitors. Ecol. Lett. 18, 916-926. https://doi.org/10.1111/ele.12468

Terseleer, N., Bruggeman, J., Lancelot, C., Gypens, N., 2014. Trait-based representation of diatom functional diversity in a plankton functional type model of the eutrophied southern North Sea. Limnology and Oceanography 59, 1958-1972. https://doi.org/10.4319/lo.2014.59.6.1958

Zwart, J.A., Solomon, C.T., Jones, S.E., 2015. Phytoplankton traits predict ecosystem function in a global set of lakes. Ecology 96, 2257-2264. https://doi.org/10.1890/14-2102.1

Follett, C.L., Dutkiewicz, S., Karl, D.M., Inomura, K., Follows, M.J., 2018. Seasonal resource conditions favor a summertime increase in North Pacific diatom-diazotroph associations. ISME J. 12, 1543-1557. https://doi.org/10.1038/s41396-017-0012-x equation-based + individual-based

equation-based

equation-based

species

equation-based

community

equation-based

community

equation-based

community

equation-based

ecosystem

equation-based

local

statistical

ecosystem

equation-based

equation-based

population

equation-based

community 
4. Trait-based models on microorganisms and soil decomposers

Bouskill, N.J., Tang, J., Riley, W.J., Brodie, E.L., 2012. Trait-based representation of biological nitrification: Model development, testing, and predicted community composition. Front. Microbiol. 3, 1-17. https://doi.org/10.3389/fmicb.2012.00364

4. Trait-based models on microorganisms and soil decomposers

Le Roux, X., Bouskill, N.J., Niboyet, A., Barthes, L., Dijkstra, P., Field, C.B., Hungate, B.A., Lerondelle, C., Pommier, T. Tang, J., Terada, A., Tourna, M., Poly, F., 2016. Predicting the responses of soil nitrite-oxidizers to multi-factorial global change: A trait-based approach. Front. Microbiol. 7, 1-13. https://doi.org/10.3389/fmicb.2016.00628

4. Trait-based models on microorganisms and soil decomposers

Song, H.-S., Thomas, D.G., Stegen, J.C., Li, M., Liu, C , Song, X., Chen, X., Fredrickson, J.K. Zachara, J.M., Scheibe, T.D. 2017. Regulation-Structured Dynamic Metabolic Model Provides a Potential Mechanism for Delayed Enzyme Response in Denitrification Process. Front. Microbiol. 8, 1-12. https://doi.org/10.3389/fmicb.2017.01866

4. Trait-based models on microorganisms and soil decomposers

Van Bellen, S., Mauquoy, D., Payne, R.J., Roland, T.P., Hughes, P.D.M., Daley, T.J., Loader, N.J., Street-Perrott, F.A., Rice, E.M., Pancotto, V.A., 2017. An alternative approach to transfer functions? Testing the performance of a functional trait-based model for testate amoebae. Palaeogeogr. Palaeoclimatol. Palaeoecol. 468, 173-183.

https://doi.org/10.1016/j.palaeo.2016.12.00505

4. Trait-based models on microorganisms and soil decomposers

Wieder, W. R., Grandy, A.S., Kallenbach, C.M. Taylor, P.G., Bonan, G.B., 2015. Representing life in the Earth system with soil microbial functional traits in the MIMICS model. Geosci. Model Dev. 8, 1789-1808. https://doi.org/10.5194/gmd-8-17892015

Trait-based models applied to terrestrial and marine ecosystems alike
Harfoot, M.B.J., Newbold, T., Tittensor, D.P., Emmott, S., Hutton, J., Lyutsarev, V., Smith, M J., Scharlemann, J.P.W., Purves, D.W., 2014. Emergent Global Patterns of Ecosystem Structure and Function from a Mechanistic General Ecosystem Model. PLoS Biol. 12. https://doi.org/10.1371/journal.pbio.1001841 
Table A1.2. Search terms and history of the literature search.

5.12 .2018

Web of Science Core Collection

TOPIC= ("trait-base*" AND model*)
Results: $772 \quad$ From: 1978

Refined by excluding Psychology, Medicine, Engineering, Business, Management, History, Industrial relations, linguistics, education, nutrition, biotechnology

Results: $619 \quad$ From: 1978

WOS Search TS $=($ "trait-base*" AND model*) NOT WC $=($ PSYCHOLOGY BIOLOGICAL OR SPORT History: SCIENCES OR NEUROSCIENCES OR CLINICAL NEUROLOGY OR BIOCHEMICAL RESEARCH METHODS OR DENTISTRY ORAL SURGERY MEDICINE OR ECONOMICS OR EDUCATION EDUCATIONAL RESEARCH OR EDUCATION SPECIAL OR ENERGY FUELS OR PSYCHOLOGY DEVELOPMENTAL OR ENGINEERING ELECTRICAL ELECTRONIC OR ENGINEERING MANUFACTURING OR GERIATRICS GERONTOLOGY OR HEALTH POLICY SERVICES OR PSYCHOLOGY CLINICAL OR CRIMINOLOGY PENOLOGY OR HISTORY OR PSYCHOLOGY SOCIAL OR HEALTH CARE SCIENCES SERVICES OR HOSPITALITY LEISURE SPORT TOURISM OR PSYCHIATRY OR METEOROLOGY ATMOSPHERIC SCIENCES OR IMAGING SCIENCE PHOTOGRAPHIC TECHNOLOGY OR INDUSTRIAL RELATIONS LABOR OR LINGUISTICS OR PSYCHOLOGY MULTIDISCIPLINARY OR VETERINARY SCIENCES OR MEDICAL INFORMATICS OR MEDICINE RESEARCH EXPERIMENTAL OR ANESTHESIOLOGY OR EDUCATION SCIENTIFIC DISCIPLINES OR NURSING OR PEDIATRICS OR PLANNING DEVELOPMENT OR PSYCHOLOGY MATHEMATICAL OR MANAGEMENT OR PUBLIC ENVIRONMENTAL OCCUPATIONAL HEALTH OR NUTRITION DIETETICS OR BUSINESS OR PHARMACOLOGY PHARMACY OR FOOD SCIENCE TECHNOLOGY OR PHYSICS FLUIDS PLASMAS OR PSYCHOLOGY EXPERIMENTAL OR PHYSICS MATHEMATICAL OR SUBSTANCE ABUSE OR WOMEN S STUDIES OR TOXICOLOGY OR POLITICAL SCIENCE OR PSYCHOLOGY APPLIED OR PSYCHOLOGY EDUCATIONAL) Indexes=SCI-EXPANDED, SSCI Timespan=All years

\footnotetext{
Web of Science Core Collection

TOPIC $=\left(\right.$ "traitbase ${ }^{* "}$ AND model*)

Results: 4

From: 2010

WOS Search TS $=\left(\right.$ "traitbase*" AND model* $\left.{ }^{*}\right)$

History:
} 


\section{Appendix. Chapter 2.}

Table A2.1. Model parameters for sensitivity analysis. Parameters are given with their description, range for Morris screening and unit. Parameters highlighted in bold have constant values.

\begin{tabular}{|c|c|c|c|}
\hline Parameter & Description & Range & Unit \\
\hline \multicolumn{4}{|c|}{ World } \\
\hline min-days-year & the minimal number of rainy days in the season possible for the specific site & $1-50$ & day \\
\hline rain-days-year-dif & the difference between the minimal and the maximal number of rainy days in the season possible for the specific site & $1-50$ & day \\
\hline rain-season & typical length of the vegetation season for the specific site & $100-200$ & day \\
\hline dew-days-before & the number of days before the first rain when dew deposition might affect plant growth & $1-30$ & day \\
\hline dew-days-after & the number of days after the last rain when dew deposition might affect plant growth & $1-30$ & day \\
\hline \multicolumn{4}{|c|}{ Patches } \\
\hline min-height & the lowest height of the simulated site & 1 & $\mathrm{~m}$ \\
\hline height-dif & the difference in height between the lowest patch and the highest patch, controls the steepness of the site & $0.1-5$ & $\mathrm{~m}$ \\
\hline sd-height & standard deviation from the mean of the absolutely smooth slope, controls the smoothness of the site & $0.01-1$ & $\mathrm{~m}$ \\
\hline av-water-min & how much soil water is available for plants at the topographic object "local minimum" after a rain event, defined as probability & $0.5-1$ & - \\
\hline av-water-dif-min-slope & how much less water is available for plants at the topographical object "slope" after a rain event in comparison to the topographical object "local minimum" & $0.01-0.5$ & - \\
\hline av-water-dif-slope-max & how much less water is available for plants at the topographical object "local maximum" after a rain event in comparison to the topographical object "slope" & $0.01-0.5$ & - \\
\hline av-water-dif-max-dew & $\begin{array}{l}\text { how much less water is available for plants at all topographical object as result of dew deposition in comparison to the topographical object "local maximum" } \\
\text { after a rain event }\end{array}$ & $0.01-0.5$ & - \\
\hline \multicolumn{4}{|c|}{ Plants (All parameters below are defined separately for each species) } \\
\hline in-ad-number & initial number of adult plants & 0 & - \\
\hline ad-biomass-max & maximum biomass a species can potentially achieve, equal to the asymptotic body size & $1-1000$ & $\mathrm{~g}$ \\
\hline ad-prob-surv & probability of a plant to survive as an entire organism & $0.1-1$ & - \\
\hline juv-biomass & a fraction of maximum biomass which determines the threshold between juvenile and adult plants & $0.1-1$ & - \\
\hline juv-surv & coefficient reducing the juvenile probability to survive relative to the adult probability & $0.1-1$ & - \\
\hline \multirow[b]{2}{*}{ ad-rgr } & \multirow{2}{*}{ relative growth rate, part of the biomass equations $\quad$ Procedure Growth } & & \\
\hline & & $0.1-1$ & $\mathrm{~g} \cdot \mathrm{g}^{-1} \cdot \mathrm{day}^{-1}$ \\
\hline & Procedure Reproduction & & \\
\hline & reproduction biomass, a threshold which plants should reach to start the production of seeds & $1-100$ & $\mathrm{~g}$ \\
\hline \multirow{2}{*}{ ad-age-repr } & age at which a plant can start reproducing under suitable conditions & $1-30$ & day \\
\hline & \multirow{2}{*}{ Procedure Competition } & & \\
\hline ad-comp-asymmetry & & 1 & - \\
\hline \multicolumn{4}{|c|}{ 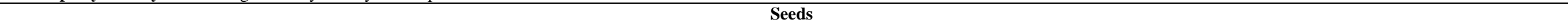 } \\
\hline in-s-number & initial number of seeds & 10000 & \\
\hline s-mass & seed weight, which lies in a species-specific range & $0.1-10$ & $\overline{\mathrm{g}}$ \\
\hline s-sp-number & $\begin{array}{c}\text { number of seeds, which lies in a species-specific range for a plant throughout the entire vegetation period } \\
\text { Procedure Germination }\end{array}$ & $1-100$ & - \\
\hline s-pr-germ & probability of seed germination, if other conditions are met & $0.1-1$ & - \\
\hline s-days-dorm & number of days that seeds should spend in dormancy; defines the possible start of germination & $1-365$ & days \\
\hline & Procedure of Dispersal & & \\
\hline s-disp-type & dispersal type a plant belongs to & 1 or 2 & - \\
\hline s-disp-shape & species-specific parameter $\beta$ in Weibull-shaped dispersal & & - \\
\hline s-disp-age & age at which a seed is dispersed after the creation & $1-1000$ & day \\
\hline s-disp-scale & species-specific parameter $\delta$ in Weibull-shaped dispersal & $0.01-10$ & - \\
\hline
\end{tabular}


Table A2.2. Model variables. The variables are given with their description and the name of the corresponding submodels

\begin{tabular}{|c|c|c|}
\hline Variable & Description & Submodel \\
\hline \multicolumn{3}{|c|}{ World } \\
\hline rainy-days & $\begin{array}{l}\text { a list of the rainy days in the current year, where the number of list items lies in a range of rainy days per year and the list item } \\
\text { itself defines the serial number of rainy days in this season }\end{array}$ & Rain \\
\hline first-rain-day & the first rainy day in a season, defined as the first number in the list of simulated rainy days & Rain \\
\hline last-rain-day & the last rainy day in a season, defined as a last number in the list of simulated rainy days & Rain \\
\hline season-correction & number of days added at the beginning of the vegetation season to simulate the effect of dew on its length & Rain \\
\hline days & one day of simulation, equal to a tick & General \\
\hline years & one year consists of 365 days, super-tick & General \\
\hline \multicolumn{3}{|c|}{ 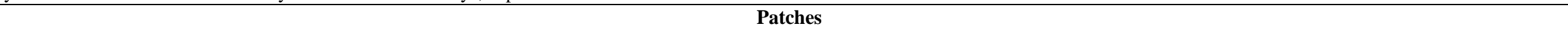 } \\
\hline height & height above sea level & Initialization \\
\hline topography & $\begin{array}{l}\text { depends on simulated heights above sea level: "slope" is a patch which has both neighbouring patches lying above and below } \\
\text { it; "local maximum" is a patch surrounded by the patches lying below it; "local minimum" is a patch surrounded by the patch- } \\
\text { es lying above it }\end{array}$ & Initialization \\
\hline number-below-neighbors & number of patches strictly below the patch of interest & Initialization \\
\hline number-above-neighbors & number of patches strictly above the patch of interest & Initialization \\
\hline p-water-availability & soil water availability, the probability that plants obtain soil water for growth and seeds for germination & Water-availability \\
\hline p-biomass_acc & accumulated biomass of competitng plants & Competition \\
\hline \multicolumn{3}{|c|}{ Plants } \\
\hline ad-number & number of adult plants & Count-plants; Output \\
\hline bio-mean & mean biomass of all adult plants of each species & Plotting; Output \\
\hline ad-biomass & current biomass; for seeds, equals seed weight & Competition; Growth; Reproduction \\
\hline & time since germination for plants and since creation for seeds & Ageing; Germination; Reproduction \\
\hline ad-radius & radius of ZOI & Competition \\
\hline ad-zoi-patches & number of patches inside the radius of $\mathrm{ZOI}$ & Competition \\
\hline ad-zoi-overlap & effective area of ZOI & Competition \\
\hline ad-zoi-resource-share & share of resources in the radius of $\mathrm{ZOI}$ & Competition \\
\hline ad-ratio-resource-area & available resources per occupied patch & Competition \\
\hline \multicolumn{3}{|c|}{ Seeds } \\
\hline s-disp-distance & seed dispersal distance according to Weibull distribution & Dispersal \\
\hline s-number-actual & number of seeds to be produced depending on current biomass & Reproduction \\
\hline
\end{tabular}


Figure A2.1. Flow-chart of the ATID-model. The flow-chart of the ATID-model demonstrates the order of the model processes. The long rectangular grey boxes represent the main blocks of the model. The rounded-rectangular blue boxes with the round-dot frame are elements of the Environment part; the rounded-rectangular green boxes with the simple frame are the elements of the Vegetation part. The rhombi represent conditions that lead to a bifurcation between two processes depending on whether the condition is fulfilled $(\mathrm{Y})$ or not $(\mathrm{N})$. The squared dark boxes are the switches that represent alternative scenarios such as dew presence or absence and different algorithms of ZOI-calculation. The processes at the level of adult plants are with the prefixes "Ad:"; the processes at the level of seeds are with the prefixes "S:". The model runs on a daily base within the vegetation season. The processes that occur only during the vegetation season are inside the upward-diagonal-pattern box with the dashed frame.

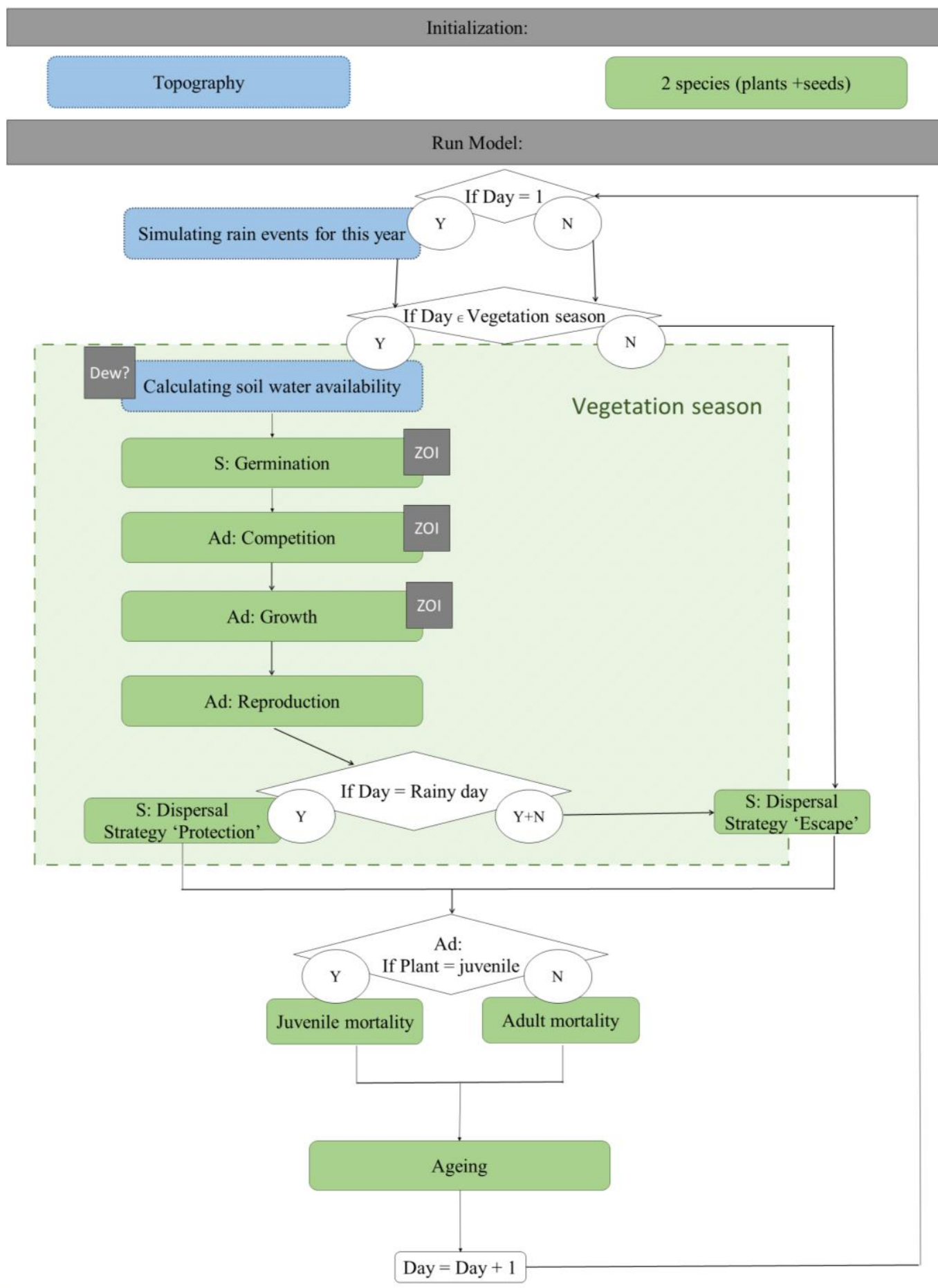




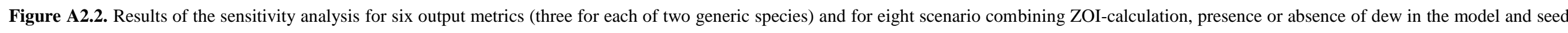

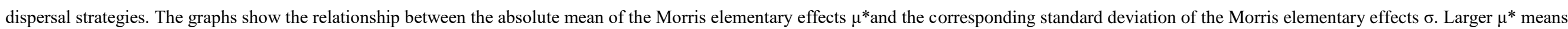

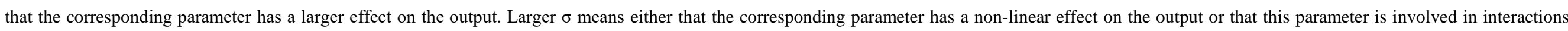

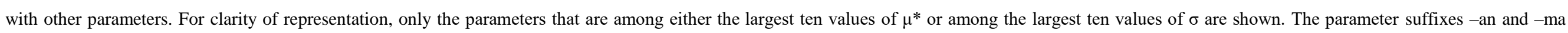
correspond to 'Anastatica' and 'Malva' (Appendix. Tab. A2.1).
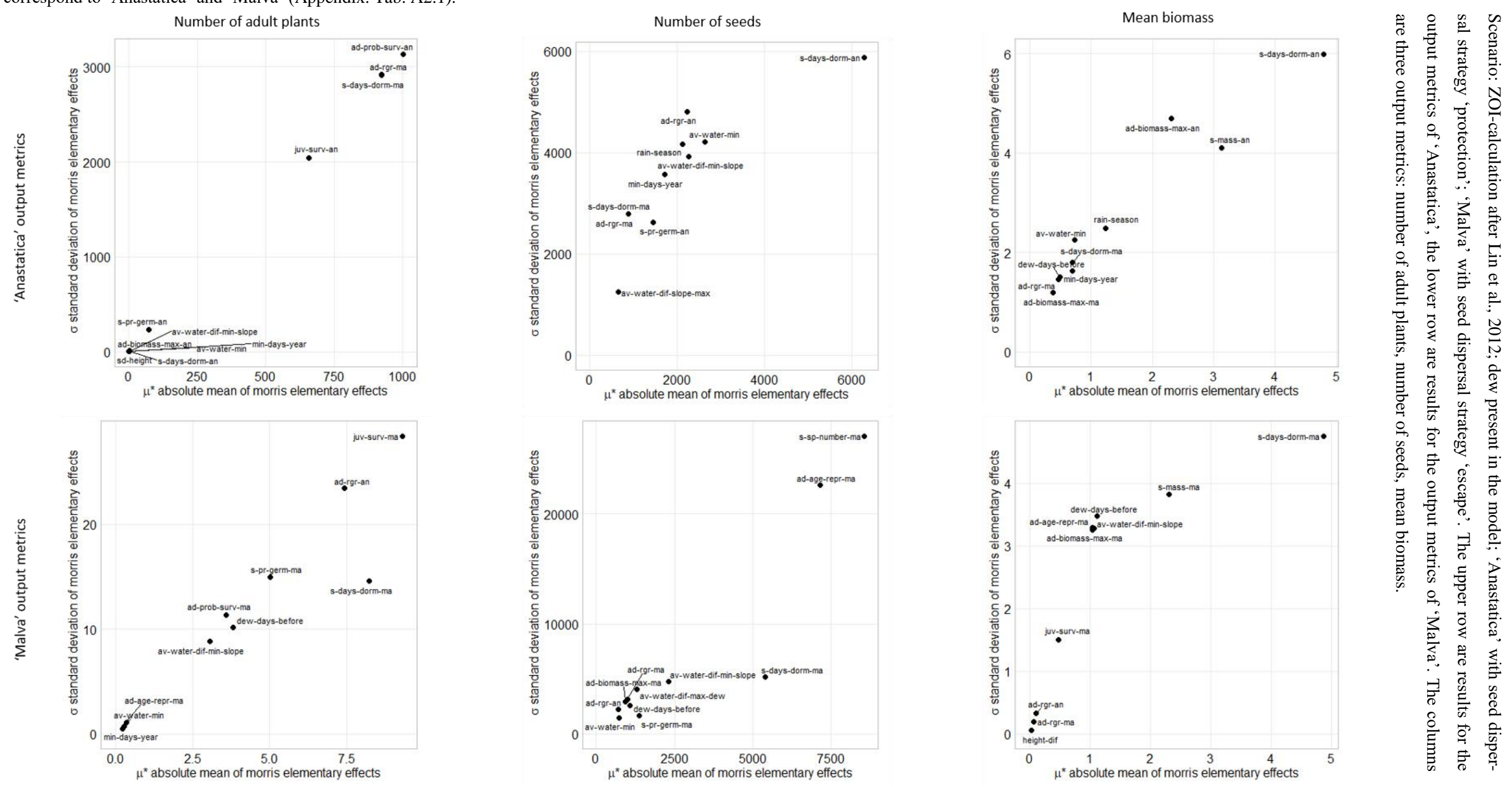


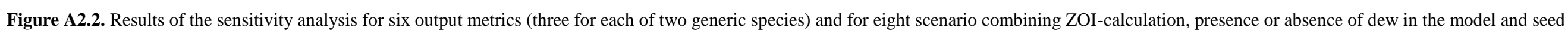

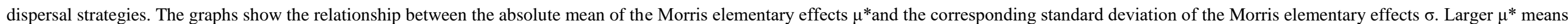

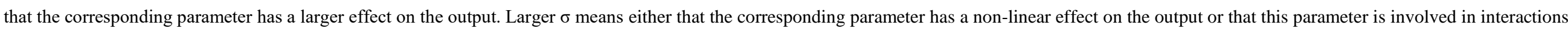

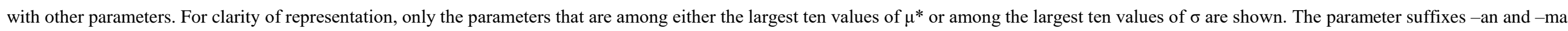
correspond to 'Anastatica' and 'Malva' (Appendix. Tab. A 2.1).
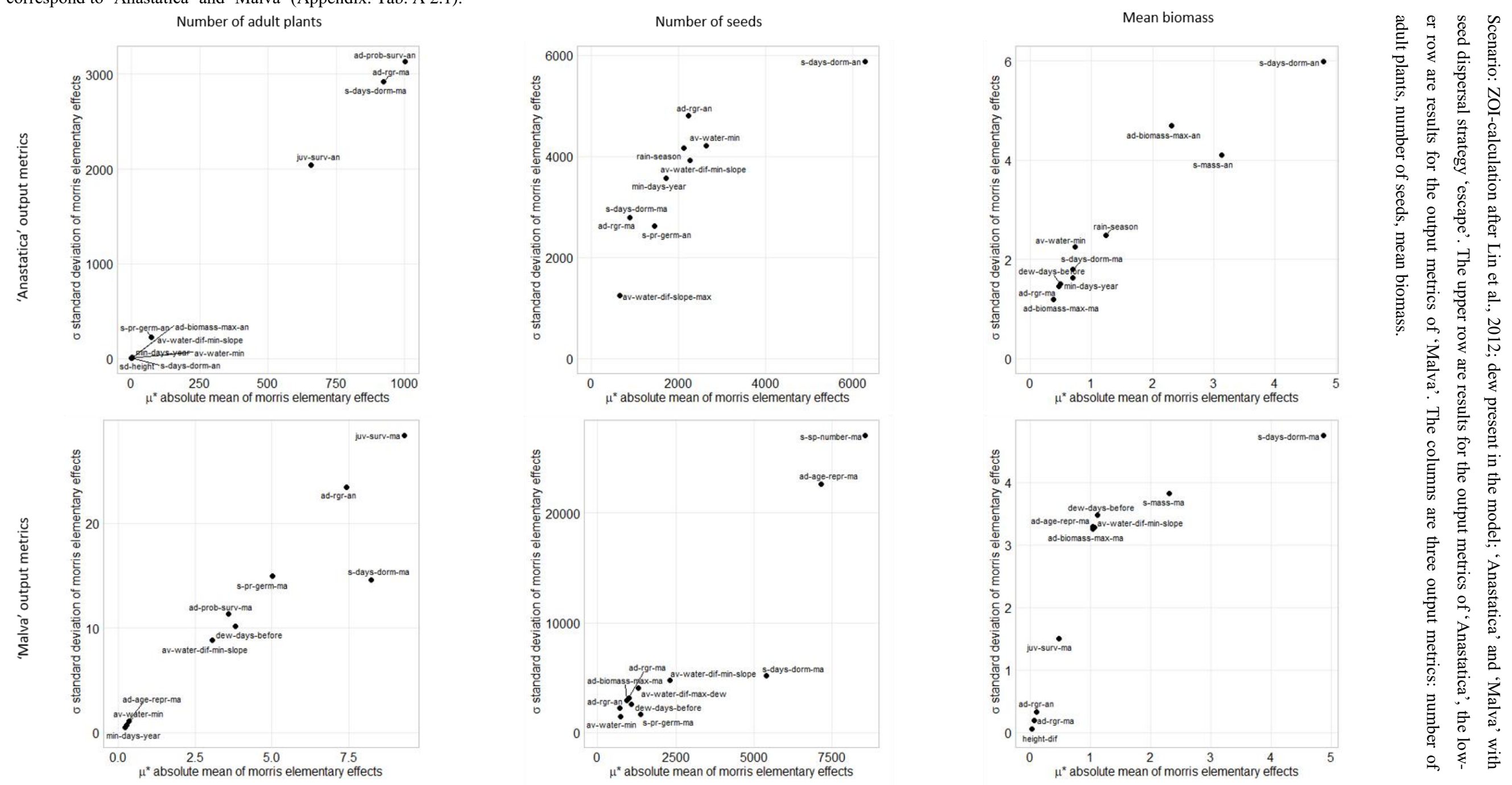


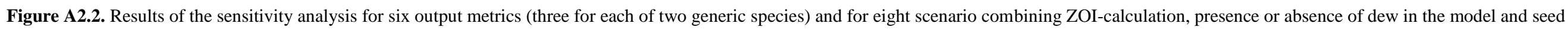

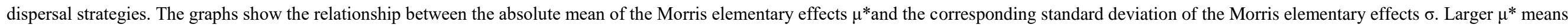

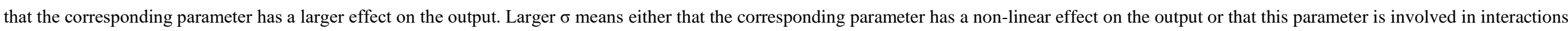

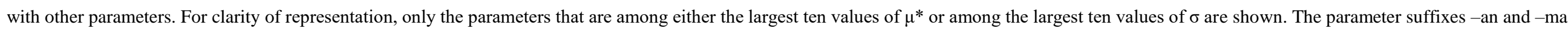
correspond to 'Anastatica' and 'Malva' (Appendix. Tab. A2.1).
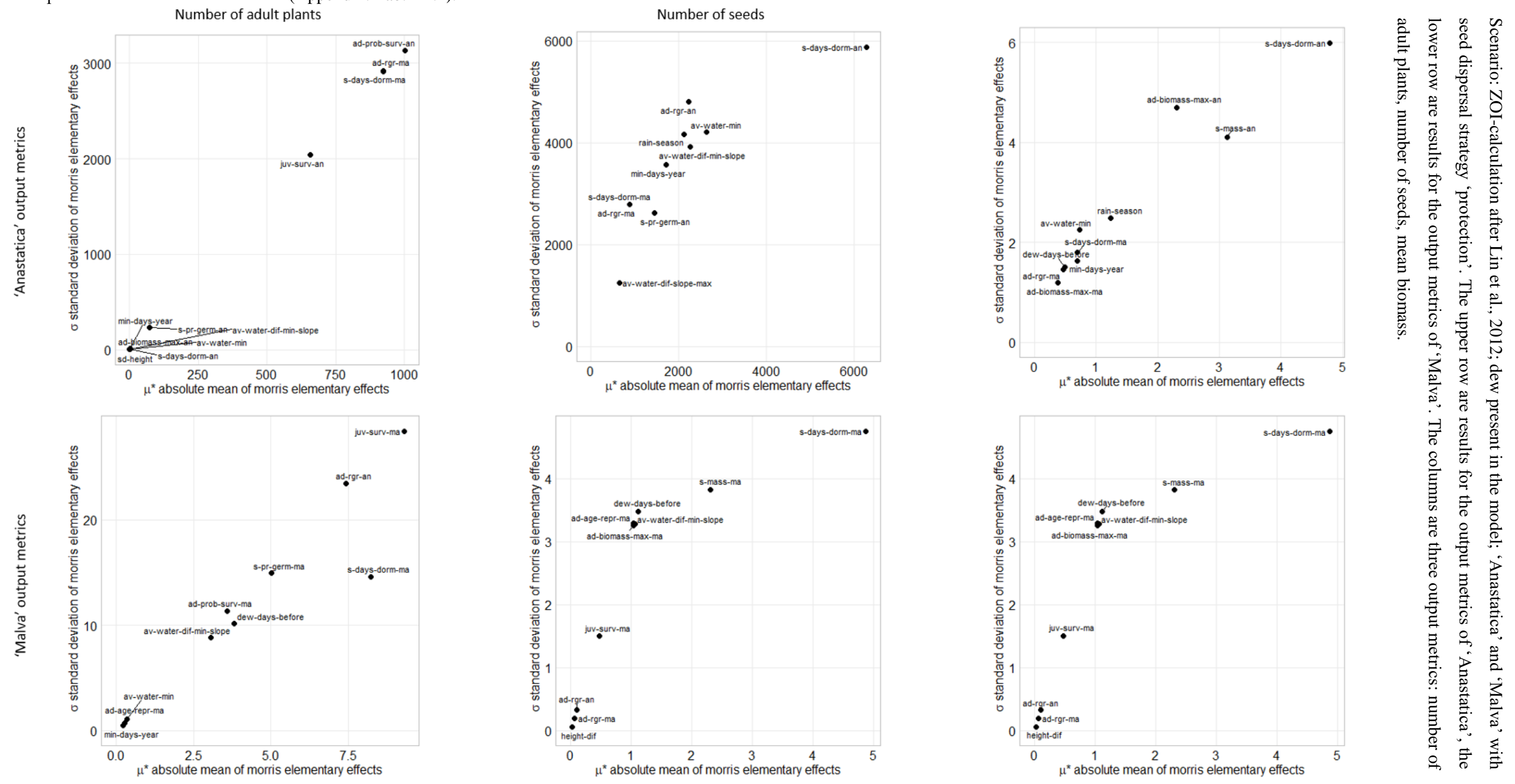

$\cong$

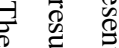

$\stackrel{\overrightarrow{0}}{\overrightarrow{0}} \overrightarrow{\mathrm{\theta}}$

ఏ 尺े

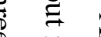

Е

o

这

垔

$\infty$

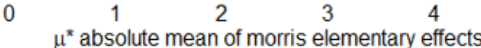




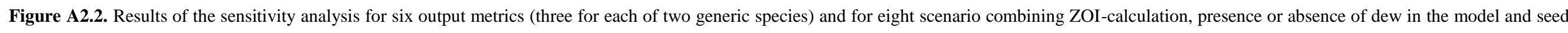

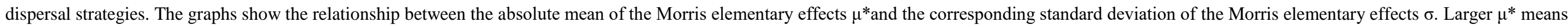

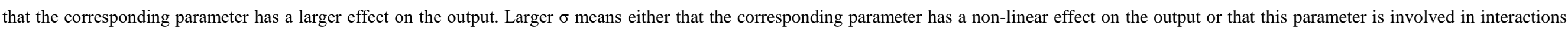

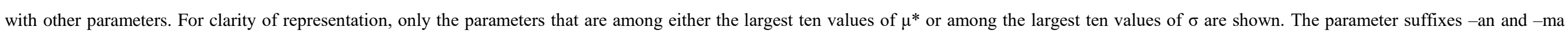
correspond to 'Anastatica' and 'Malva' (Appendix. Tab. A2.1).
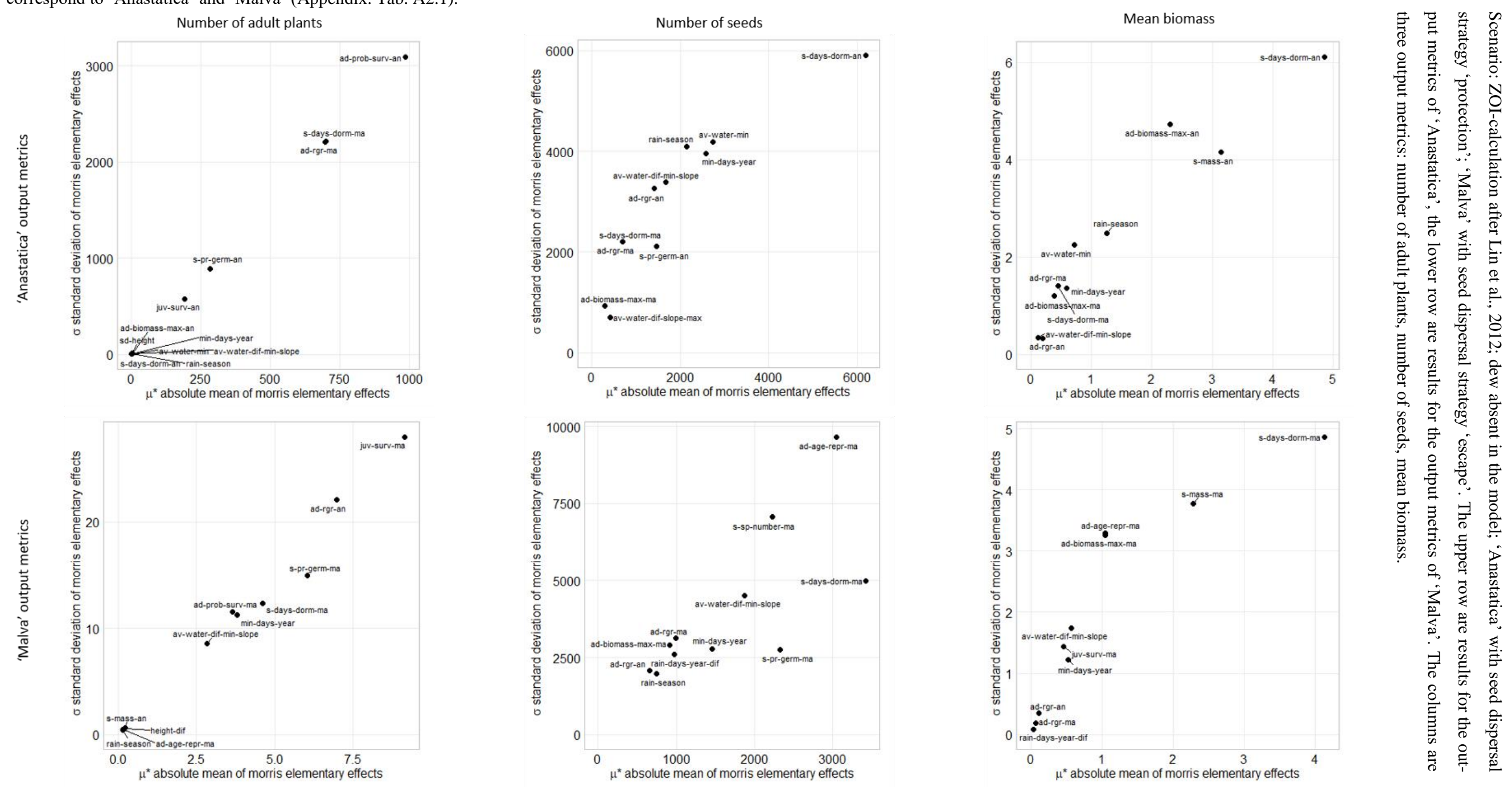

$\begin{array}{cc}1000 & 2000 \\ \mu^{*} \text { absolute mean of morris elementary effects }\end{array}$

$\mu^{*}$ absolute mean of morris elementary effects 


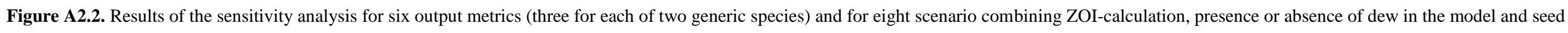

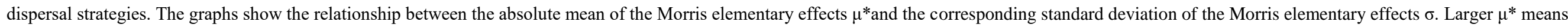

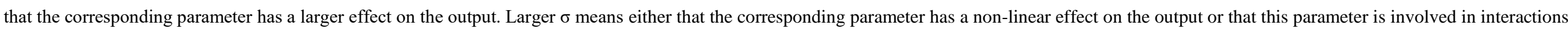

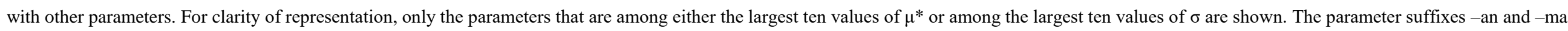
correspond to 'Anastatica' and 'Malva' (Appendix. Tab. A2.1).
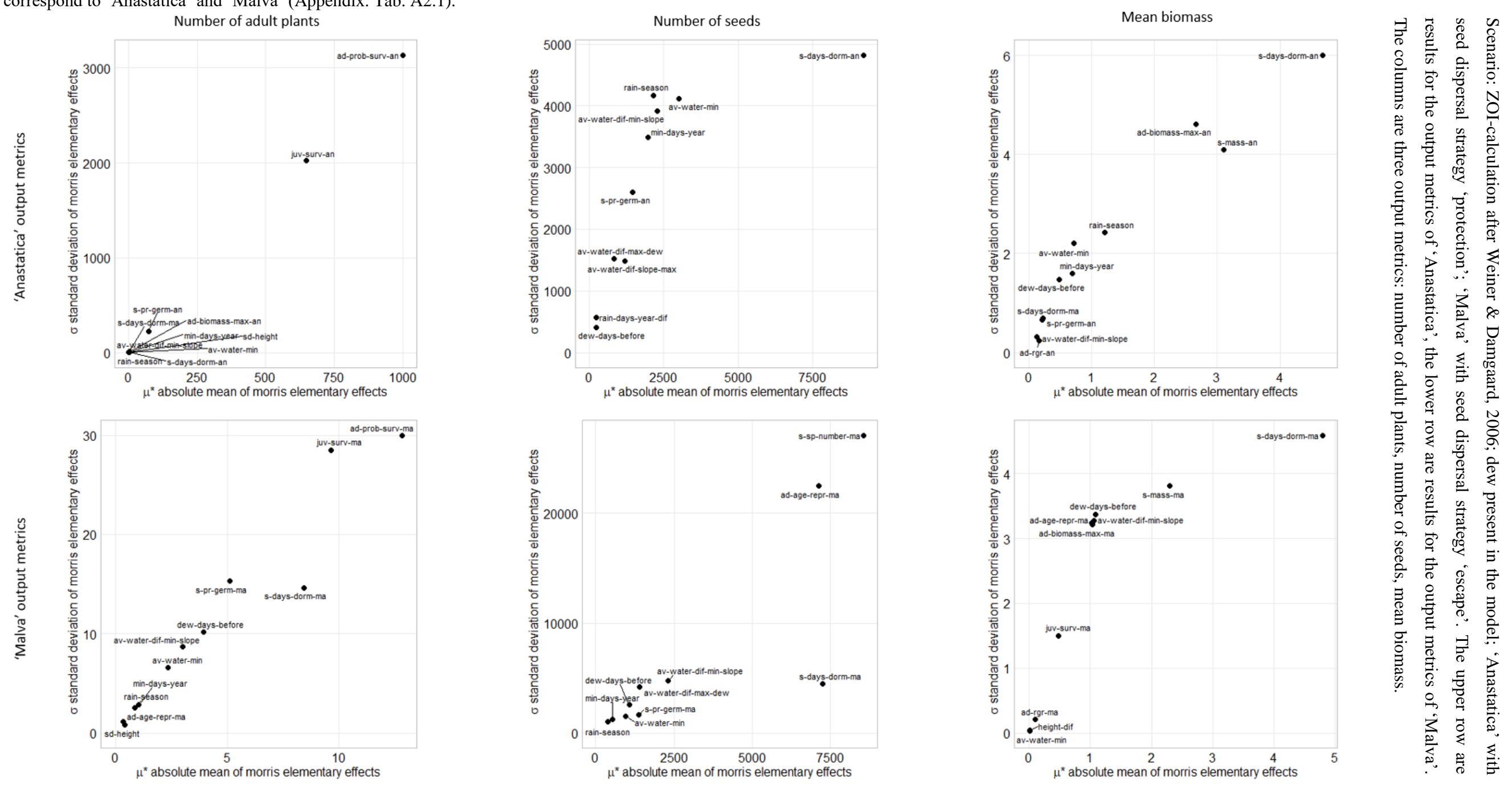


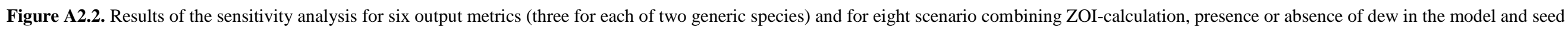

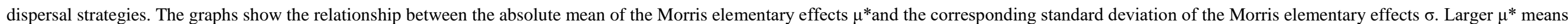

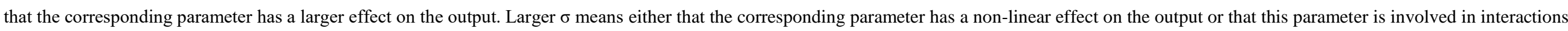

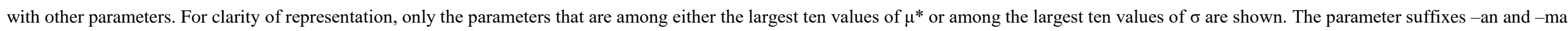
correspond to 'Anastatica' and 'Malva' (Appendix. Tab. A2.1).
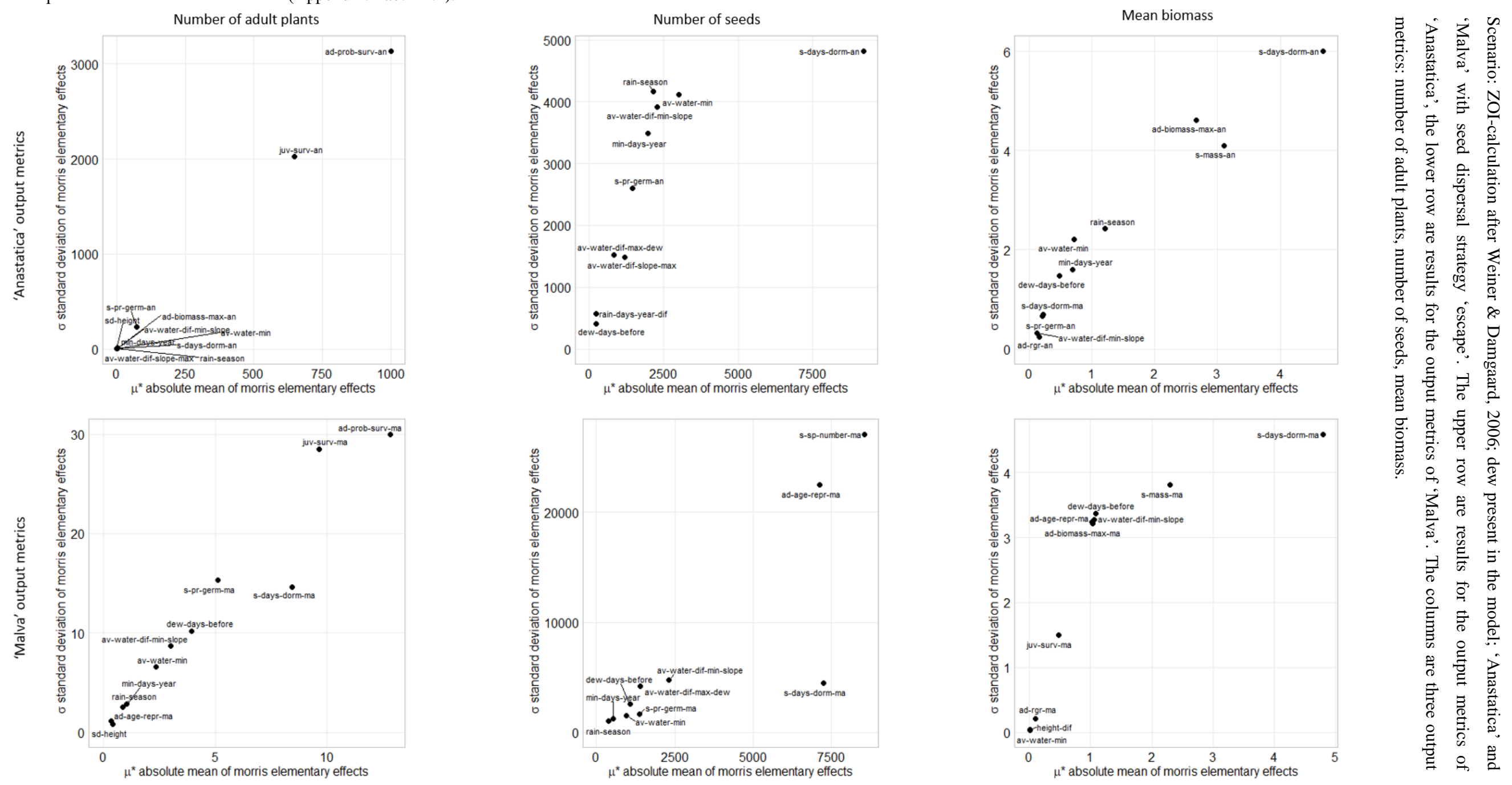


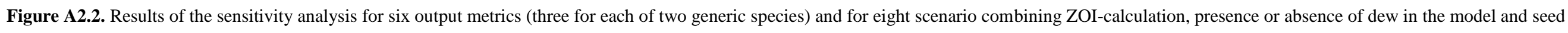

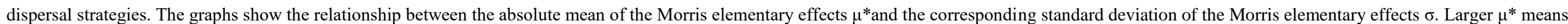

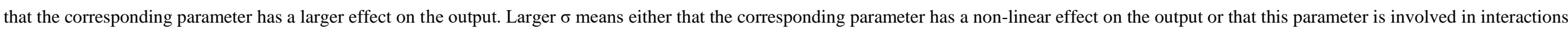

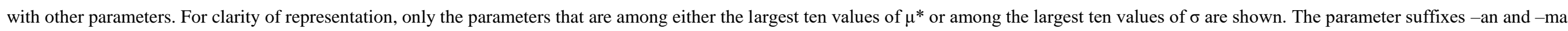
correspond to 'Anastatica' and 'Malva' (Appendix. Tab. A2.1).
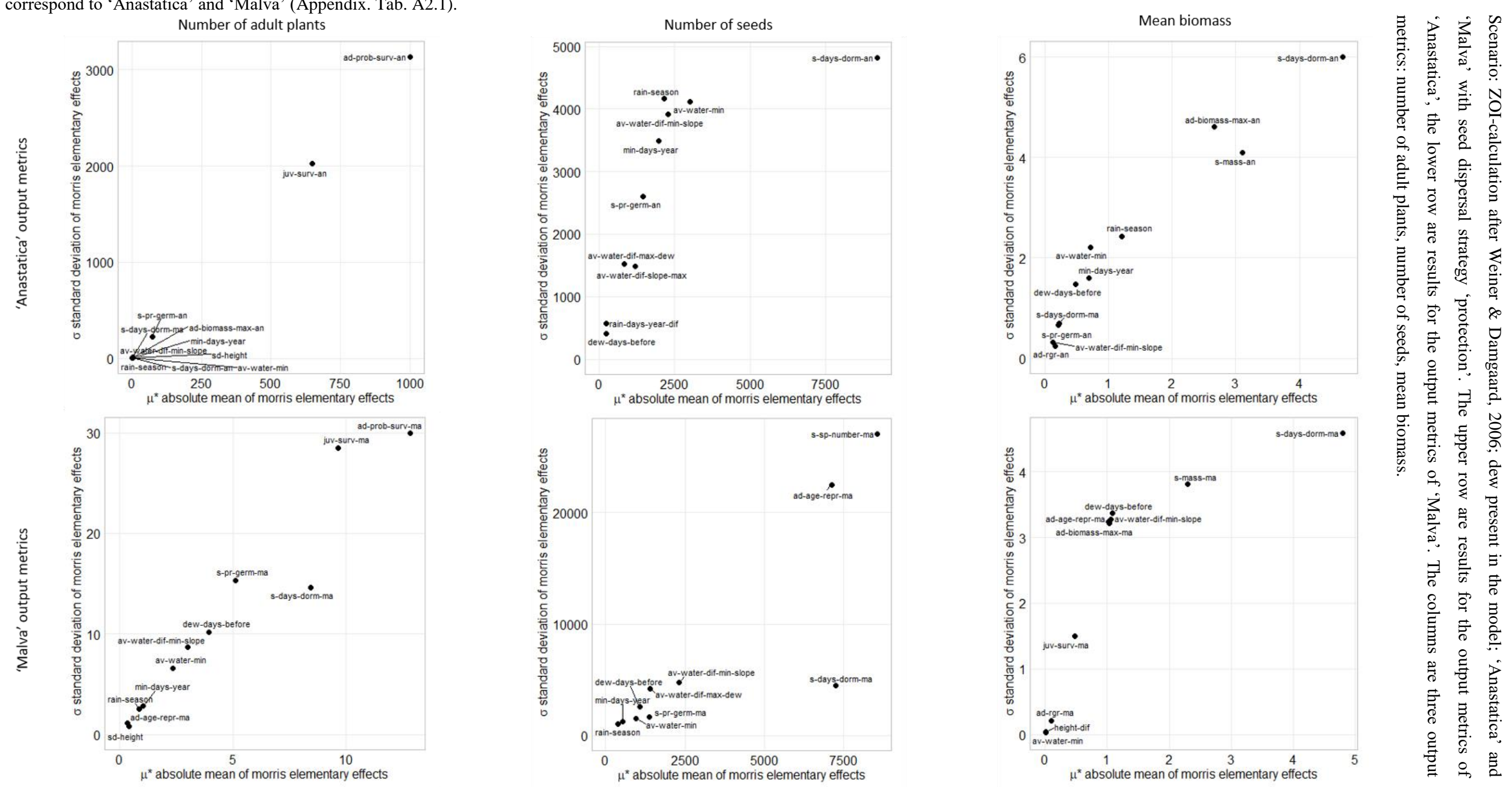


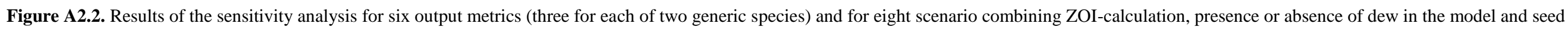

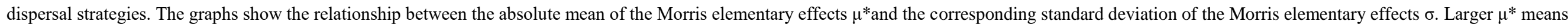

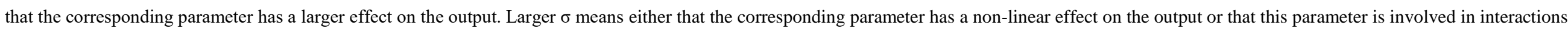

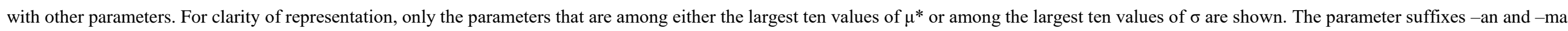
correspond to 'Anastatica' and 'Malva' (Appendix. Tab. A2.1).
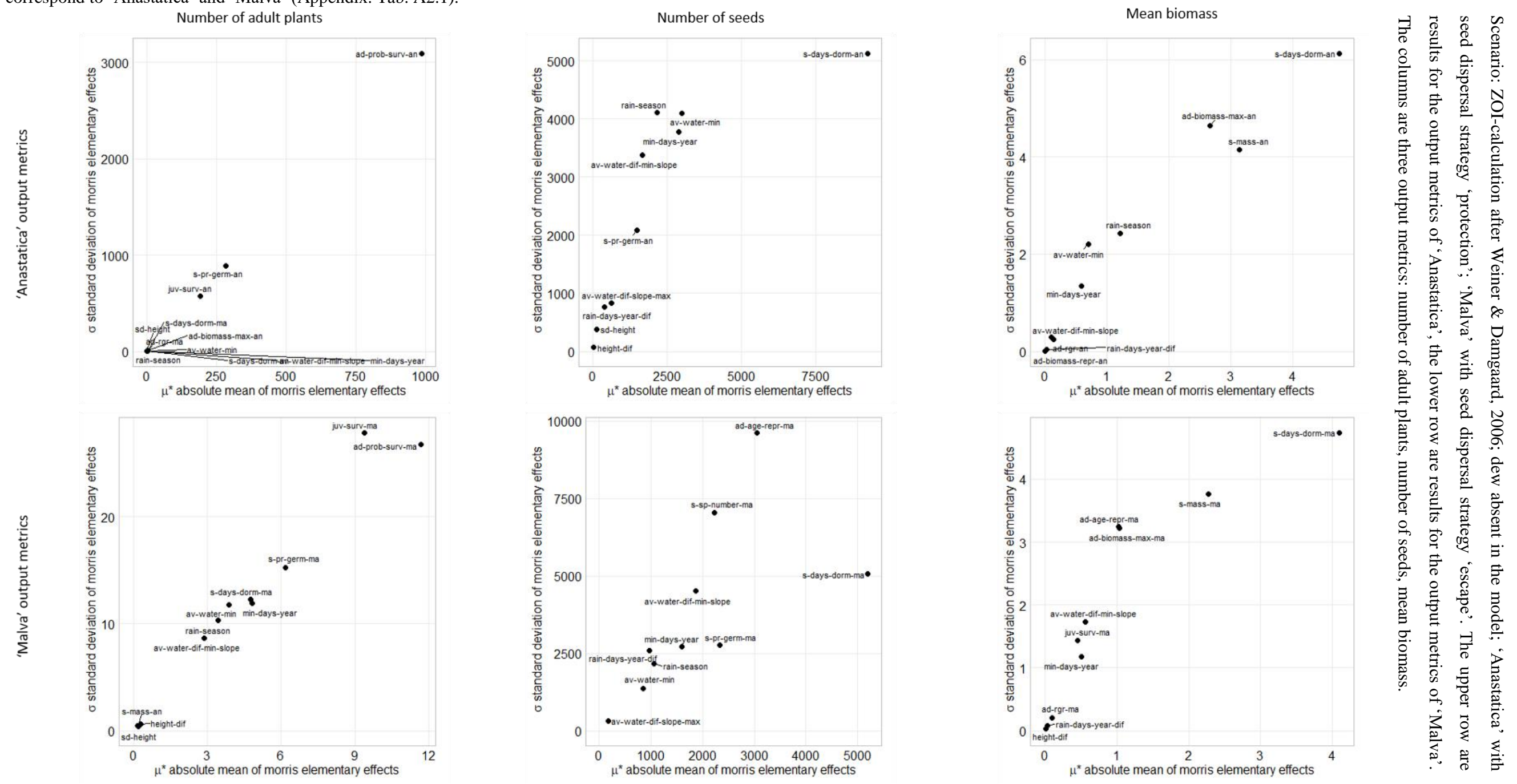


\section{Appendix. Chapter 3.}

Table A3.1. Model parameters for simulation of Meishar community. Parameters are given with their description, value, unit and the source of their value.

\begin{tabular}{|c|c|c|c|c|}
\hline Parameter & Description & Value & Unit & Source \\
\hline \multicolumn{5}{|c|}{ World } \\
\hline min-days-year & $\begin{array}{l}\text { the minimum number of rainy days in } \\
\text { the season possible for the specific site }\end{array}$ & 12 & & meteorological station \\
\hline max-days-year & $\begin{array}{l}\text { the maximum number of rainy days in } \\
\text { the season possible for the specific site }\end{array}$ & 42 & & meteorological station \\
\hline rain-season & $\begin{array}{l}\text { typical length of the vegetation season } \\
\text { for the specific site }\end{array}$ & 120 & & meteorological station \\
\hline $\begin{array}{l}\text { dew-days- } \\
\text { before }\end{array}$ & $\begin{array}{l}\text { the number of days before the first rain } \\
\text { when dew deposition might affect plant } \\
\text { growth }\end{array}$ & 5 & & educated guess \\
\hline dew-days-after & $\begin{array}{l}\text { the number of days after the last rain } \\
\text { when dew deposition might affect plant } \\
\text { growth }\end{array}$ & 5 & & educated guess \\
\hline \multicolumn{5}{|c|}{ Patches } \\
\hline min-height & the lowest height of the simulated site & 1 & $\mathrm{~m}$ & digital elevation model \\
\hline max-height & the highest height of the simulated site & 6 & $\mathrm{~m}$ & digital elevation model \\
\hline sd-height & $\begin{array}{l}\text { standard deviation from the mean of the } \\
\text { absolutely smooth slope, controls the } \\
\text { smoothness of the site }\end{array}$ & 0.05 & $\mathrm{~m}$ & digital elevation model \\
\hline av-water-min & $\begin{array}{l}\text { how much soil water is available for } \\
\text { plants at the topographic object "local } \\
\text { minimum" after a rain event, defined as } \\
\text { probability }\end{array}$ & 0.95 & & educated guess \\
\hline av-water-slope & $\begin{array}{l}\text { how much soil water is available for } \\
\text { plants at the topographical object "slope" } \\
\text { after a rain event }\end{array}$ & 0.9 & & educated guess \\
\hline av-water-max & $\begin{array}{l}\text { how much soil water is available for } \\
\text { plants at the topographical object "local } \\
\text { maximum" after a rain event }\end{array}$ & 0.85 & & educated guess \\
\hline av-water-dew & $\begin{array}{l}\text { how much soil water is available for } \\
\text { plants at all topographical object as re- } \\
\text { sult of dew deposition }\end{array}$ & 0.65 & & educated guess \\
\hline
\end{tabular}

\section{ANASTATICA}

Plants

\begin{tabular}{|c|c|}
\hline in-ad-number & initial number of adult plants \\
\hline $\begin{array}{l}\text { ad-biomass- } \\
\max \end{array}$ & $\begin{array}{l}\text { maximum biomass a species can poten- } \\
\text { tially achieve, equal to the asymptotic } \\
\text { body size }\end{array}$ \\
\hline ad-prob-surv & $\begin{array}{l}\text { probability of a plant to survive as an } \\
\text { entire organism }\end{array}$ \\
\hline
\end{tabular}

juv-biomass

juv-surv

ad-rgr

ad-biomass-

repr

ad-age-repr reproduction biomass, a threshold whic
plants should reach to start the production of seeds

age at which a plant can start reproducing under suitable conditions a fraction of maximum biomass which determines the threshold between juvenile and adult plants coefficient reducing the juvenile probability to survive relative to the adult probability

0
493
0.5

\section{Procedure Growth}

relative growth rate, part of the biomass equations
2.1 $\mathrm{g} \cdot \mathrm{g}^{-}$
$1 \cdot$ day $^{-1}$

Procedure Reproduction $\mathrm{g}$

educated guess

g calibration

Hegazy, A. K., Kabiel, H. F., Alatar, A. A., \& Lovett-Doust, J. (2013). Plasticity in dynamics and hygrochastic persistence in Anastatica hierochuntica L. (Brassicaceae) populations under simulated rainfall treatments. Pol. J. Ecol, 61(3), 493-504.

calibration

calibration

educated guess

Hegazy, A. K., Kabiel, H. F., Alatar, A. A., \& Lovett-Doust, J. (2013). Plasticity in dynamics and hygrochastic persistence in Anastatica hierochuntica L. (Brassicaceae) populations under 


\begin{tabular}{|c|c|c|c|c|}
\hline Parameter & Description & Value & Unit & Source \\
\hline & & & & $\begin{array}{l}\text { simulated rainfall treatments. Pol. J. Ecol, 61(3), } \\
493-504 .\end{array}$ \\
\hline \multicolumn{5}{|c|}{ Procedure Competition } \\
\hline ad-comp- & degree of asymmetry of competition & 1 & - & below-ground competition \\
\hline \multicolumn{5}{|c|}{ Seeds } \\
\hline in-s-number & initial number of seeds & 9000 & _ & calibration \\
\hline s-mass & $\begin{array}{l}\text { seed weight, which lies in a species- } \\
\text { specific range }\end{array}$ & 1 & $\mathrm{~g}$ & $\begin{array}{l}\text { El-Keblawy, A., \& Gairola, S. (2017). Dormancy } \\
\text { regulating chemicals alleviate innate seed dor- } \\
\text { mancy and promote germination of desert annu- } \\
\text { als. Journal of Plant Growth Regulation, 36(2), } \\
\text { 300-311. }\end{array}$ \\
\hline s-sp-number & $\begin{array}{l}\text { number of seeds, which lies in a species- } \\
\text { specific range for a plant throughout the } \\
\text { entire vegetation period }\end{array}$ & 1000 & - & $\begin{array}{l}\text { Hegazy, A. K., Kabiel, H. F., Alatar, A. A., \& } \\
\text { Lovett-Doust, J. (2013). Plasticity in dynamics } \\
\text { and hygrochastic persistence in Anastatica hiero- } \\
\text { chuntica L. (Brassicaceae) populations under } \\
\text { simulated rainfall treatments. Pol. J. Ecol, 61(3), } \\
\text { 493-504. }\end{array}$ \\
\hline \multicolumn{5}{|c|}{ Procedure Germination } \\
\hline s-pr-germ & $\begin{array}{l}\text { probability of seed germination, if other } \\
\text { conditions are met }\end{array}$ & 0.8 & - & $\begin{array}{l}\text { Bhatt, A., Phondani, P. C., Phartyal, S. S., Santo, } \\
\text { A., \& Gallacher, D. (2017). Influence of aerial } \\
\text { seed banks on germination response in three de- } \\
\text { sert plant species. Journal of Plant Ecology, } \\
10(6), 994-1000 .\end{array}$ \\
\hline s-days-dorm & $\begin{array}{l}\text { number of days that seeds should spend } \\
\text { in dormancy; defines the possible start of } \\
\text { germination }\end{array}$ & 0 & days & $\begin{array}{l}\text { Viemont, J. D., \& Crabbé, J. (Eds.). (2000). Dor- } \\
\text { mancy in plants: from whole plant behaviour to } \\
\text { cellular control. }\end{array}$ \\
\hline \multicolumn{5}{|c|}{ Procedure of Dispersal } \\
\hline s-disp-type & dispersal type a plant belongs to & 2 & - & $\begin{array}{l}\text { Type } 2 \text { describes a mechanism when seeds are } \\
\text { dispersed in portions only during a rain event }\end{array}$ \\
\hline s-disp-shape & $\begin{array}{l}\text { species-specific parameter } \beta \text { in Weibull- } \\
\text { shaped dispersal }\end{array}$ & 2.5 & - & $\begin{array}{l}\text { Kelly, N., Cousens, R. D., Taghizadeh, M. S., } \\
\text { Hanan, J. S., \& Mouillot, D. (2013). Plants as } \\
\text { populations of release sites for seed dispersal: a } \\
\text { structural-statistical analysis of the effects of } \\
\text { competition on Raphanus raphanistrum. Journal } \\
\text { of ecology, 101(4), 878-888. }\end{array}$ \\
\hline s-disp-scale & $\begin{array}{l}\text { species-specific parameter } \delta \text { in Weibull- } \\
\text { shaped dispersal }\end{array}$ & 0.4 & - & $\begin{array}{l}\text { Kelly, N., Cousens, R. D., Taghizadeh, M. S., } \\
\text { Hanan, J. S., \& Mouillot, D. (2013). Plants as } \\
\text { populations of release sites for seed dispersal: a } \\
\text { structural-statistical analysis of the effects of } \\
\text { competition on Raphanus raphanistrum. Journal } \\
\text { of ecology, 101(4), 878-888. }\end{array}$ \\
\hline s-disp-age & $\begin{array}{l}\text { age at which a seed is dispersed after the } \\
\text { creation }\end{array}$ & 5 & day & $\begin{array}{l}\text { Hegazy, A. K., Kabiel, H. F., Alatar, A. A., \& } \\
\text { Lovett-Doust, J. (2013). Plasticity in dynamics } \\
\text { and hygrochastic persistence in Anastatica hiero- } \\
\text { chuntica L. (Brassicaceae) populations under } \\
\text { simulated rainfall treatments. Pol. J. Ecol, 61(3), } \\
\text { 493-504. }\end{array}$ \\
\hline \multicolumn{5}{|c|}{ MALVA } \\
\hline \multicolumn{5}{|c|}{ Plants } \\
\hline in-ad-number & initial number of adult plants & 0 & - & educated guess \\
\hline $\begin{array}{l}\text { ad-biomass- } \\
\max \end{array}$ & $\begin{array}{l}\text { maximum biomass a species can poten- } \\
\text { tially achieve, equal to the asymptotic } \\
\text { body size }\end{array}$ & 474 & g & calibration \\
\hline ad-prob-surv & $\begin{array}{l}\text { probability of a plant to survive as an } \\
\text { entire organism despite the death be- } \\
\text { cause of biomass loss, e.g. mechanic } \\
\text { damages }\end{array}$ & 0.95 & - & $\begin{array}{l}\text { Golan, S., Faraj, T., Rahamim, E., Zemach, H., } \\
\text { Lifshitz, D., Singer, A., ... \& Gati, E. M. (2016). } \\
\text { The effect of petroleum hydrocarbons on seed } \\
\text { germination, development and survival of wild } \\
\text { and cultivated plants in extreme desert soil. Int. J. } \\
\text { Agric. Environ. Res., 2, 1743-1767. }\end{array}$ \\
\hline juv-biomass & $\begin{array}{l}\text { a fraction of maximum biomass which } \\
\text { determines the threshold between juve- } \\
\text { nile and adult plants }\end{array}$ & 0.03 & - & calibration \\
\hline
\end{tabular}




\begin{tabular}{|c|c|c|c|c|}
\hline Parameter & Description & Value & Unit & Source \\
\hline juv-surv & $\begin{array}{l}\text { coefficient reducing the juvenile proba- } \\
\text { bility to survive relative to the adult } \\
\text { probability }\end{array}$ & 0.95 & - & calibration \\
\hline \multicolumn{5}{|c|}{ Procedure Growth } \\
\hline ad-rgr & $\begin{array}{l}\text { relative growth rate, part of the biomass } \\
\text { equations }\end{array}$ & 3.5 & $\begin{array}{l}\mathrm{g} \cdot \mathrm{g}^{-} \\
\stackrel{1}{\text { day }^{-1}}\end{array}$ & calibration \\
\hline \multicolumn{5}{|c|}{ Procedure Reproduction } \\
\hline $\begin{array}{l}\text { ad-biomass- } \\
\text { repr }\end{array}$ & $\begin{array}{l}\text { reproduction biomass, a threshold which } \\
\text { plants should reach to start the produc- } \\
\text { tion of seeds }\end{array}$ & 3 & $\mathrm{~g}$ & educated guess \\
\hline ad-age-repr & $\begin{array}{l}\text { age at which a plant can start reproduc- } \\
\text { ing under suitable conditions }\end{array}$ & 21 & day & $\begin{array}{l}\text { UWA369 - Agro-ecology of small-flowered mal- } \\
\text { low (Malva parviflora) in Western Australian } \\
\text { farming systems }\end{array}$ \\
\hline \multicolumn{5}{|c|}{ Procedure Competition } \\
\hline ad-comp- & degree of asymmetry of competition & 1 & - & below-ground competition \\
\hline \multicolumn{5}{|c|}{ Seeds } \\
\hline in-s-number & initial number of seeds & 9000 & - & calibration \\
\hline s-mass & $\begin{array}{l}\text { seed weight, which lies in a species- } \\
\text { specific range }\end{array}$ & 0.2 & $\mathrm{~g}$ & $\begin{array}{l}\text { Proctor, V. W. (1968). Long-distance dispersal of } \\
\text { seeds by retention in digestive tract of birds. Sci- } \\
\text { ence, } 160(3825), 321-322\end{array}$ \\
\hline s-sp-number & $\begin{array}{l}\text { number of seeds, which lies in a species- } \\
\text { specific range for a plant throughout the } \\
\text { entire vegetation period }\end{array}$ & 3000 & - & $\begin{array}{l}\text { Michael, P. J., Steadman, K. J., Plummer, J. A., \& } \\
\text { Vercoe, P. (2006). Sheep rumen digestion and } \\
\text { transmission of weedy Malva parviflora seeds. } \\
\text { Australian Journal of Experimental Agriculture, } \\
46(10), 1251-1256 .\end{array}$ \\
\hline \multicolumn{5}{|c|}{ Procedure Germination } \\
\hline s-pr-germ & $\begin{array}{l}\text { probability of seed germination, if other } \\
\text { conditions are met }\end{array}$ & 0.45 & - & $\begin{array}{l}\text { Chauhan, B. S., Gill, G., \& Preston, C. (2006). } \\
\text { Factors affecting seed germination of little mal- } \\
\text { low (Malva parviflora) in southern Australia. } \\
\text { Weed Science, 54(6), 1045-1050. }\end{array}$ \\
\hline s-days-dorm & $\begin{array}{l}\text { number of days that seeds should spend } \\
\text { in dormancy; defines the possible start of } \\
\text { germination }\end{array}$ & 30 & days & $\begin{array}{l}\text { Michael, P. J., Steadman, K. J., \& Plummer, J. A. } \\
\text { (2007). Seed development in Malva parviflora: } \\
\text { onset of germinability, dormancy and desiccation } \\
\text { tolerance. Australian Journal of Experimental } \\
\text { Agriculture, } 47(6), 683-688 .\end{array}$ \\
\hline \multicolumn{5}{|c|}{ Procedure of Dispersal } \\
\hline s-disp-type & dispersal type a plant belongs to & 1 & - & $\begin{array}{l}\text { Type } 1 \text { describes a mechanism when seeds are } \\
\text { dispersed unrelated to rain events }\end{array}$ \\
\hline s-disp-shape & $\begin{array}{l}\text { species-specific parameter } \beta \text { in Weibull- } \\
\text { shaped dispersal }\end{array}$ & 2.5 & - & $\begin{array}{l}\text { Kelly, N., Cousens, R. D., Taghizadeh, M. S., } \\
\text { Hanan, J. S., \& Mouillot, D. (2013). Plants as } \\
\text { populations of release sites for seed dispersal: a } \\
\text { structural-statistical analysis of the effects of } \\
\text { competition on Raphanus raphanistrum. Journal } \\
\text { of ecology, 101(4),878-888. }\end{array}$ \\
\hline s-disp-scale & $\begin{array}{l}\text { species-specific parameter } \delta \text { in Weibull- } \\
\text { shaped dispersal }\end{array}$ & 0.4 & - & $\begin{array}{l}\text { Kelly, N., Cousens, R. D., Taghizadeh, M. S., } \\
\text { Hanan, J. S., \& Mouillot, D. (2013). Plants as } \\
\text { populations of release sites for seed dispersal: a } \\
\text { structural-statistical analysis of the effects of } \\
\text { competition on Raphanus raphanistrum. Journal } \\
\text { of ecology, 101(4), 878-888. }\end{array}$ \\
\hline s-disp-age & $\begin{array}{l}\text { age at which a seed is dispersed after the } \\
\text { creation }\end{array}$ & 21 & day & $\begin{array}{l}\text { UWA369 - Agro-ecology of small-flowered mal- } \\
\text { low (Malva parviflora) in Western Australian } \\
\text { farming systems, 2005, } \\
\text { https://grdc.com.au/research/reports/report?id=66 } \\
3\end{array}$ \\
\hline
\end{tabular}

1. Topographic information is derived from the digital elevation model. The data was collected by Nir Krintza (Ben-Gurion University, Israel) with a DJI drone (https://www.dji.com/phantom-3-adv) and a mapping program pix4d mapper (https://www.pix4d.com/product/pix4dmapper-photogrammetry-software)

2. Educated guess is based on either field observations, common sense or common knowledge from the literature that cannot be quantified precisely and thus directly cited. Calibration stays for values obtained in the calibration procedure with the R package 'nlrx', the output numbers of plants are interpolated from field measurements. 
Figure A3.1. Results of simulation runs for the species with the protective-competition strategy (black) and with the escapecolonization strategy (grey) over simulated time for the neutral scenario corresponding to the 'Meishar' site.

1) Number of plant individuals in ten simulation runs shown together (a) and separately (b)
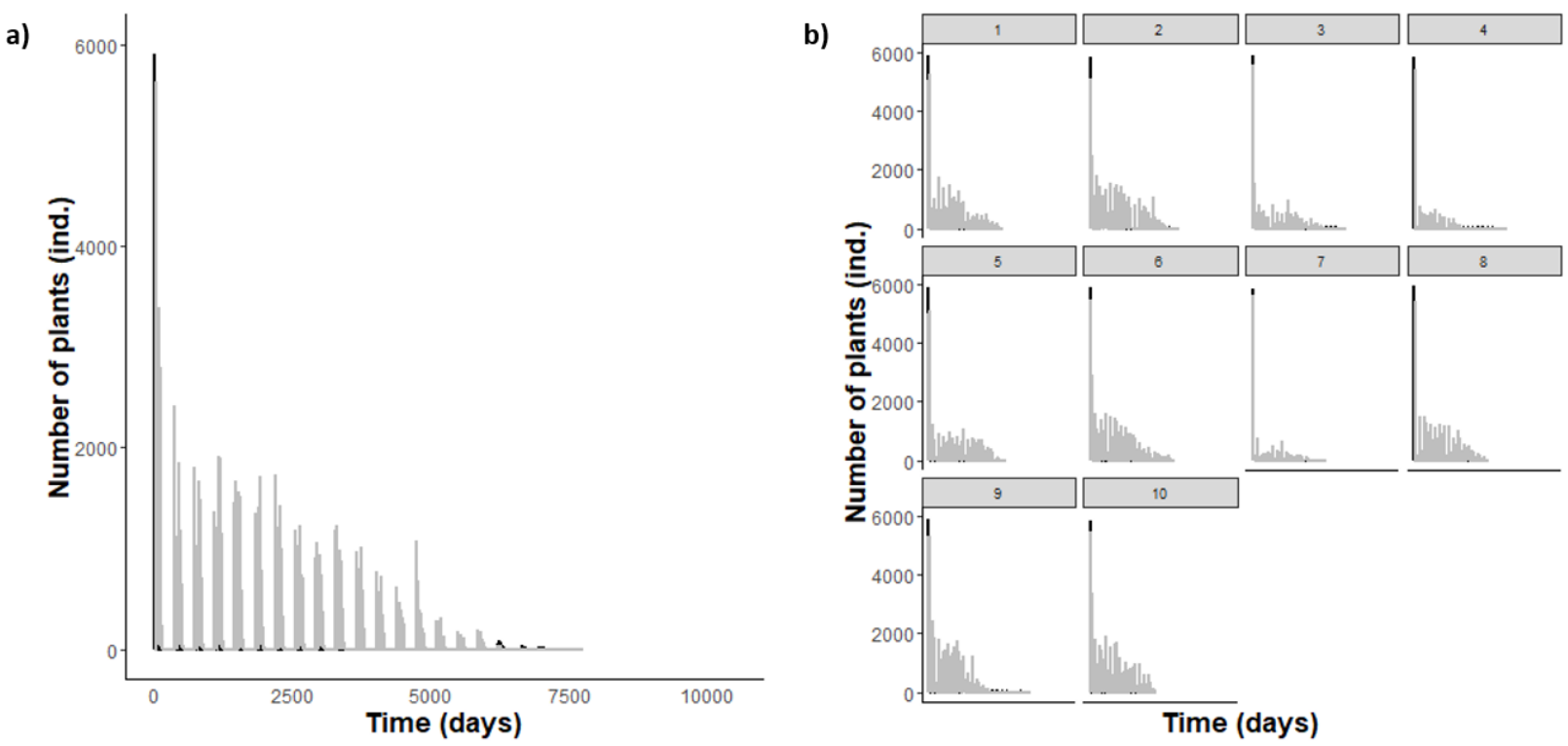

2) Number of seeds in ten simulation runs shown together (a) and separately (b)

a)

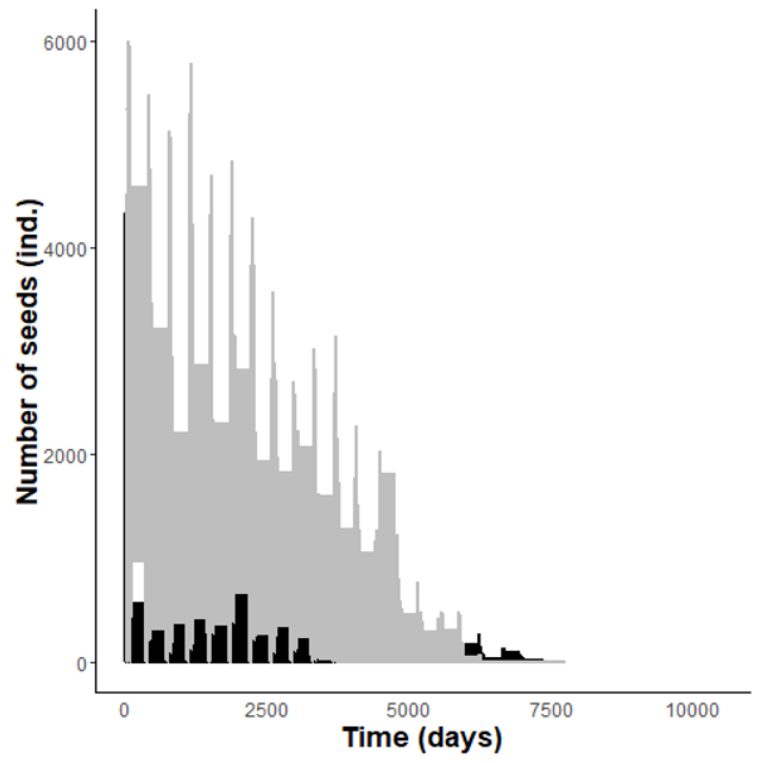

b)

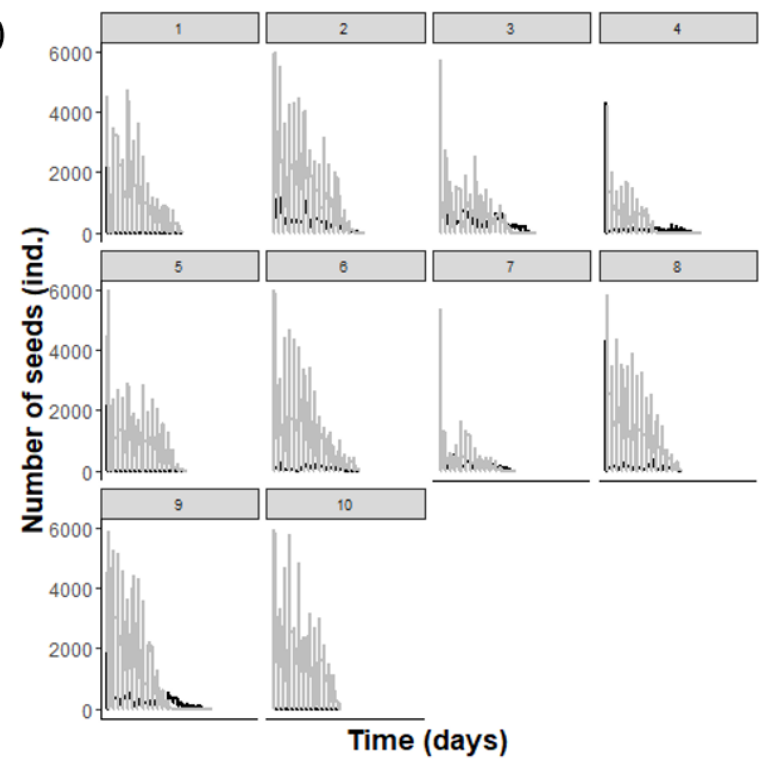


Figure A3.1. Results of simulation runs for the species with the protective-competition strategy (black) and with the escapecolonization strategy (grey) over simulated time for the neutral scenario corresponding to the 'Meishar' site.

3) Mean biomass (g) of plant individuals in ten simulation runs shown together (a) and separately (b)
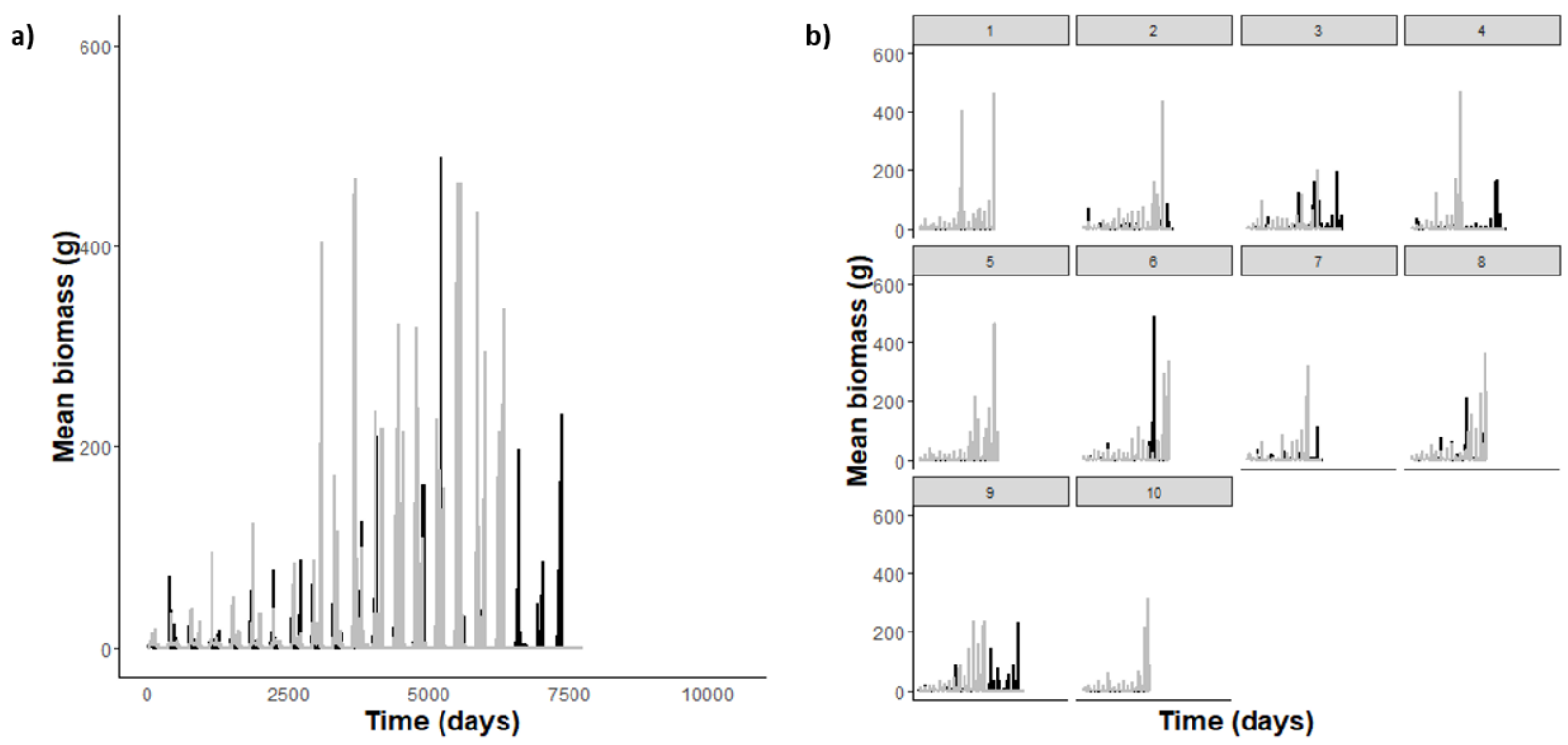

4) Proportion of the number of plants (a), number of seeds (b) and mean biomass of plants (c)
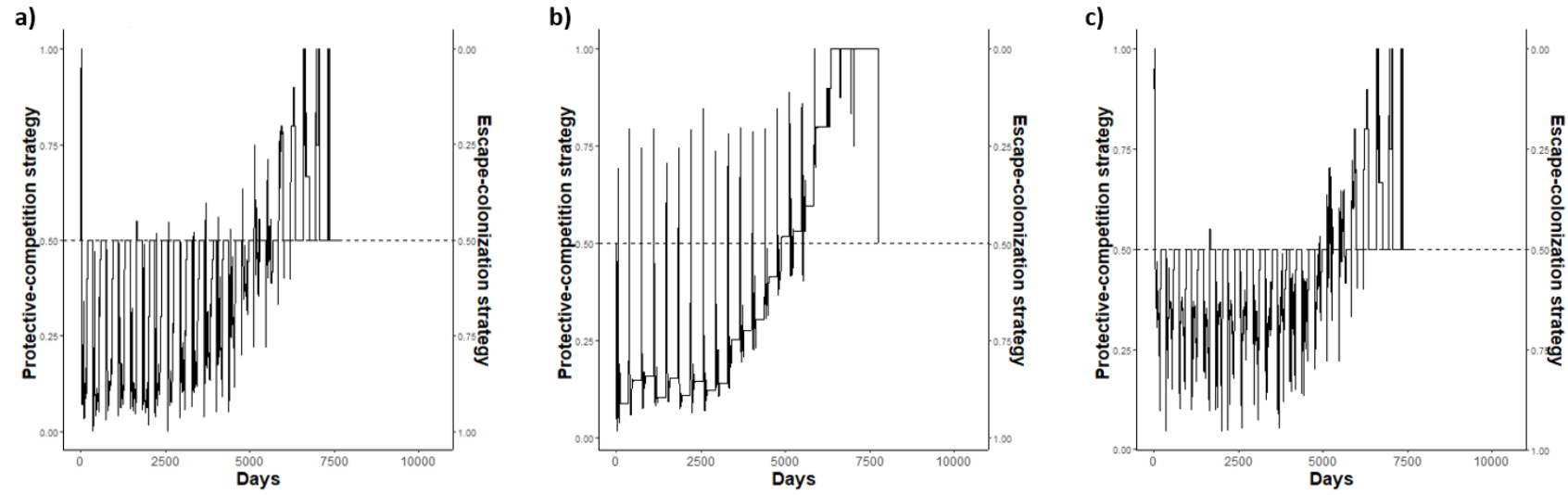
Figure A3.2. Results of simulation runs for the species with the protective-competition strategy (black) and with the escapecolonization strategy (grey) over simulated time in four scenarios (A: low temporal and spatial heterogeneity, B: low temporal heterogeneity and high spatial heterogeneity, C: high temporal heterogeneity and low spatial heterogeneity, D: high temporal and spatial heterogeneity).

1) Number of plant individuals in ten simulation runs
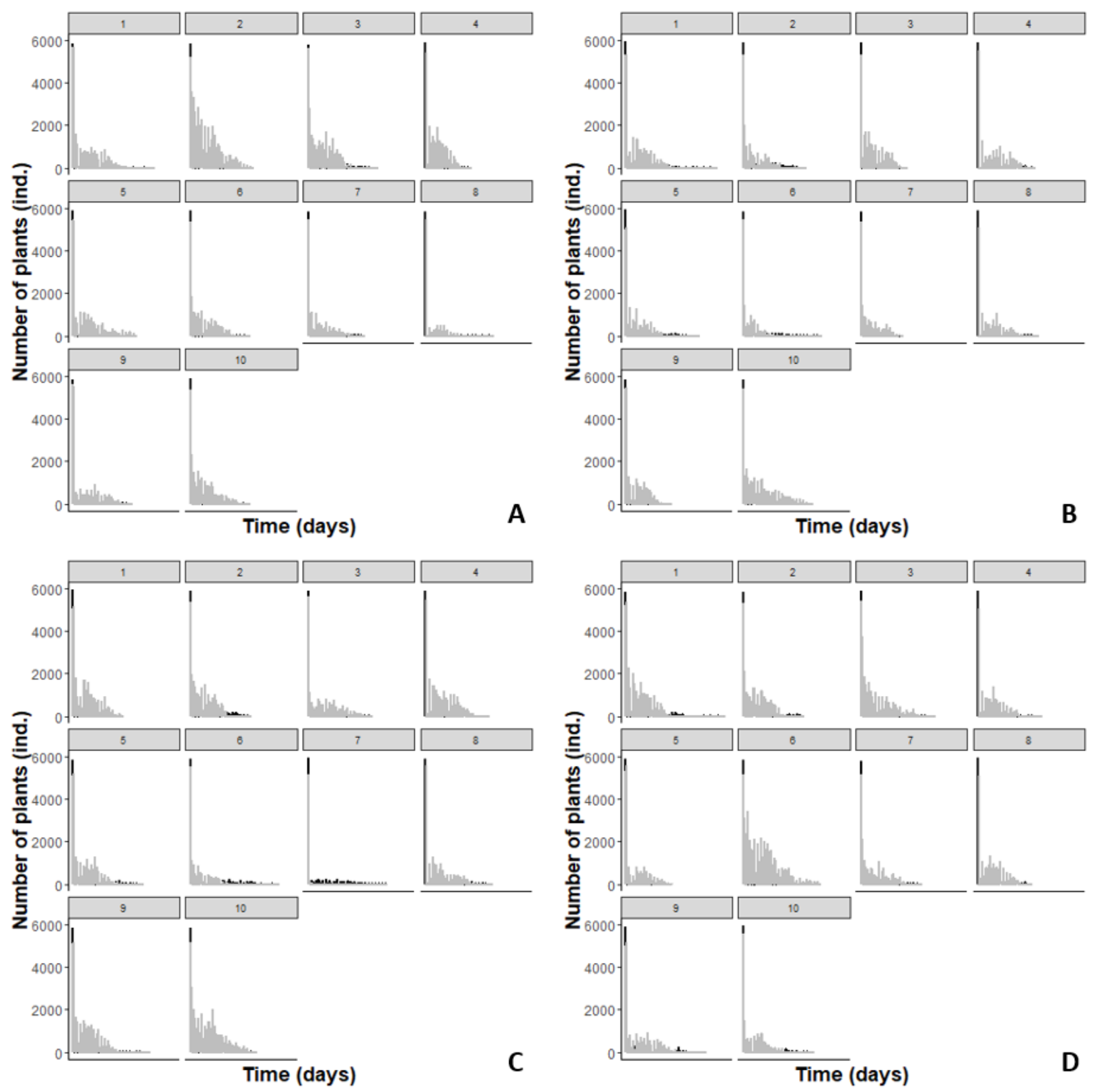
Figure A3.2. Results of simulation runs for the species with the protective-competition strategy (black) and with the escapecolonization strategy (grey) over simulated time in four scenarios (A: low temporal and spatial heterogeneity, B: low temporal heterogeneity and high spatial heterogeneity, C: high temporal heterogeneity and low spatial heterogeneity, D: high temporal and spatial heterogeneity).

2) Number of seeds in ten simulation runs
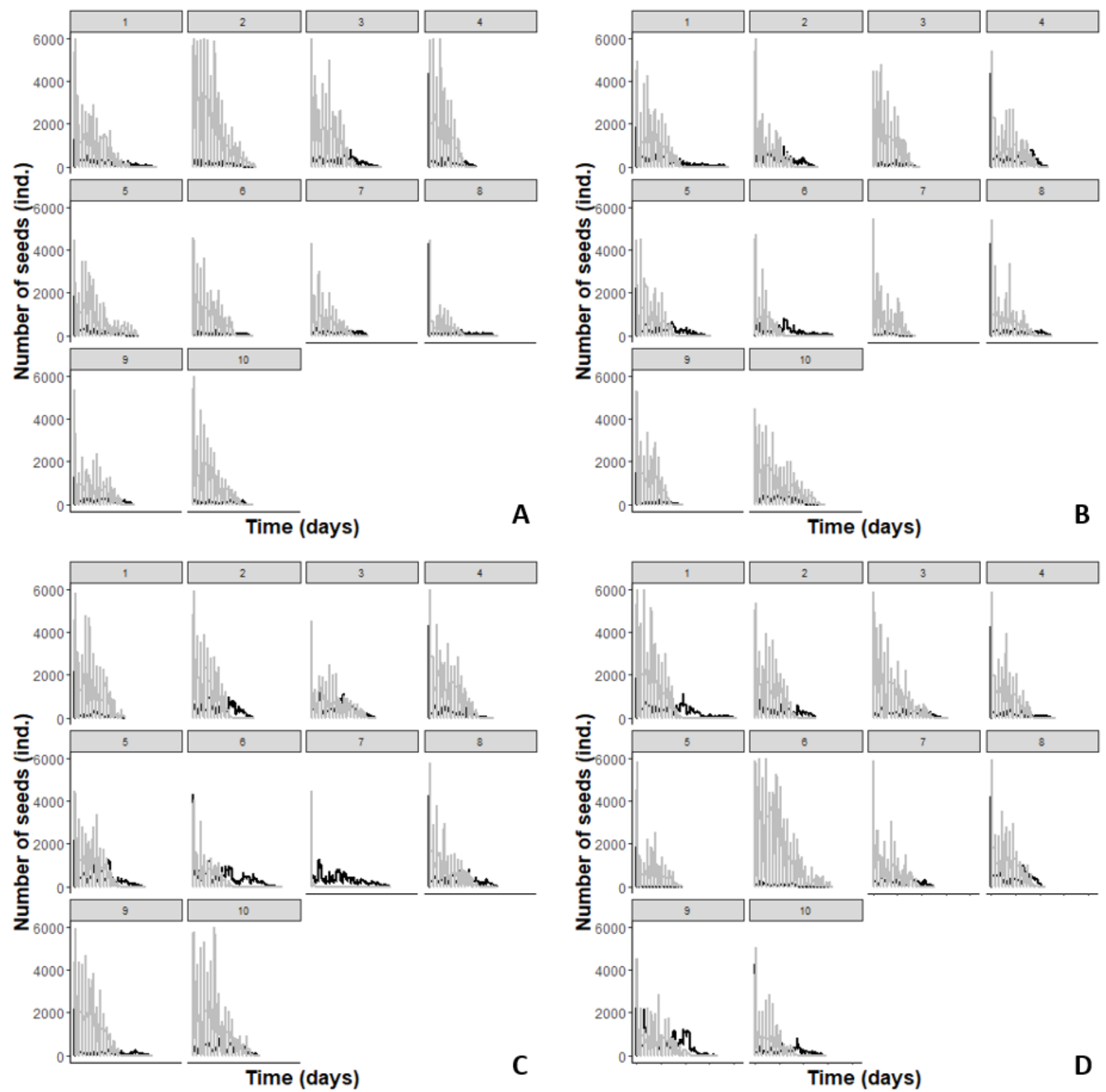
Figure A3.2. Results of simulation runs for the species with the protective-competition strategy (black) and with the escapecolonization strategy (grey) over simulated time in four scenarios (A: low temporal and spatial heterogeneity, B: low temporal heterogeneity and high spatial heterogeneity, C: high temporal heterogeneity and low spatial heterogeneity, D: high temporal and spatial heterogeneity).

3) Mean biomass ( $\mathrm{g}$ ) of plant individuals in ten simulation runs
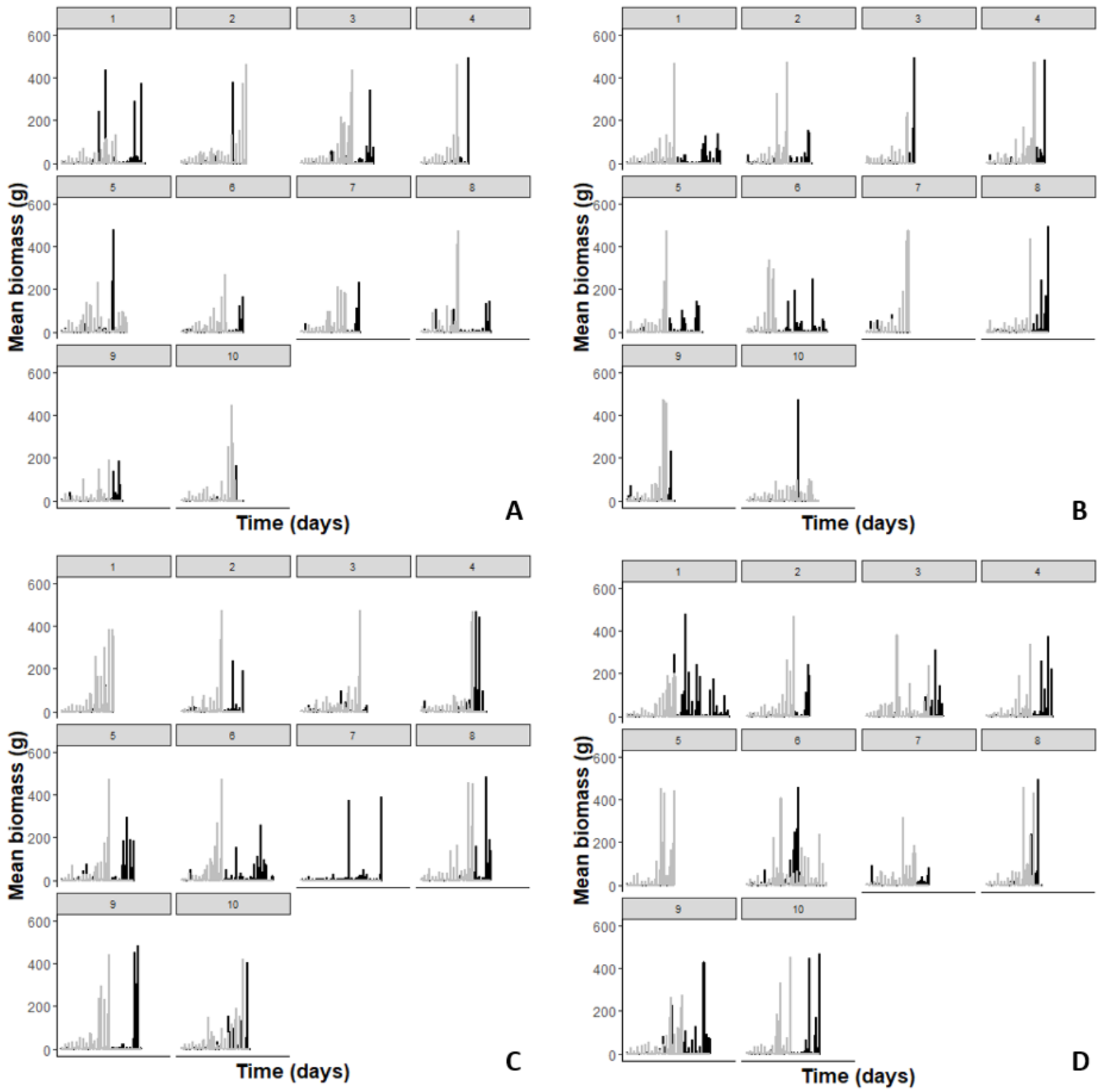
Figure A3.2. Results of simulation runs for the species with the protective-competition strategy (black) and with the escapecolonization strategy (grey) over simulated time in four scenarios (A: low temporal and spatial heterogeneity, B: low temporal heterogeneity and high spatial heterogeneity, C: high temporal heterogeneity and low spatial heterogeneity, D: high temporal and spatial heterogeneity).

4) Proportion of the number of plants
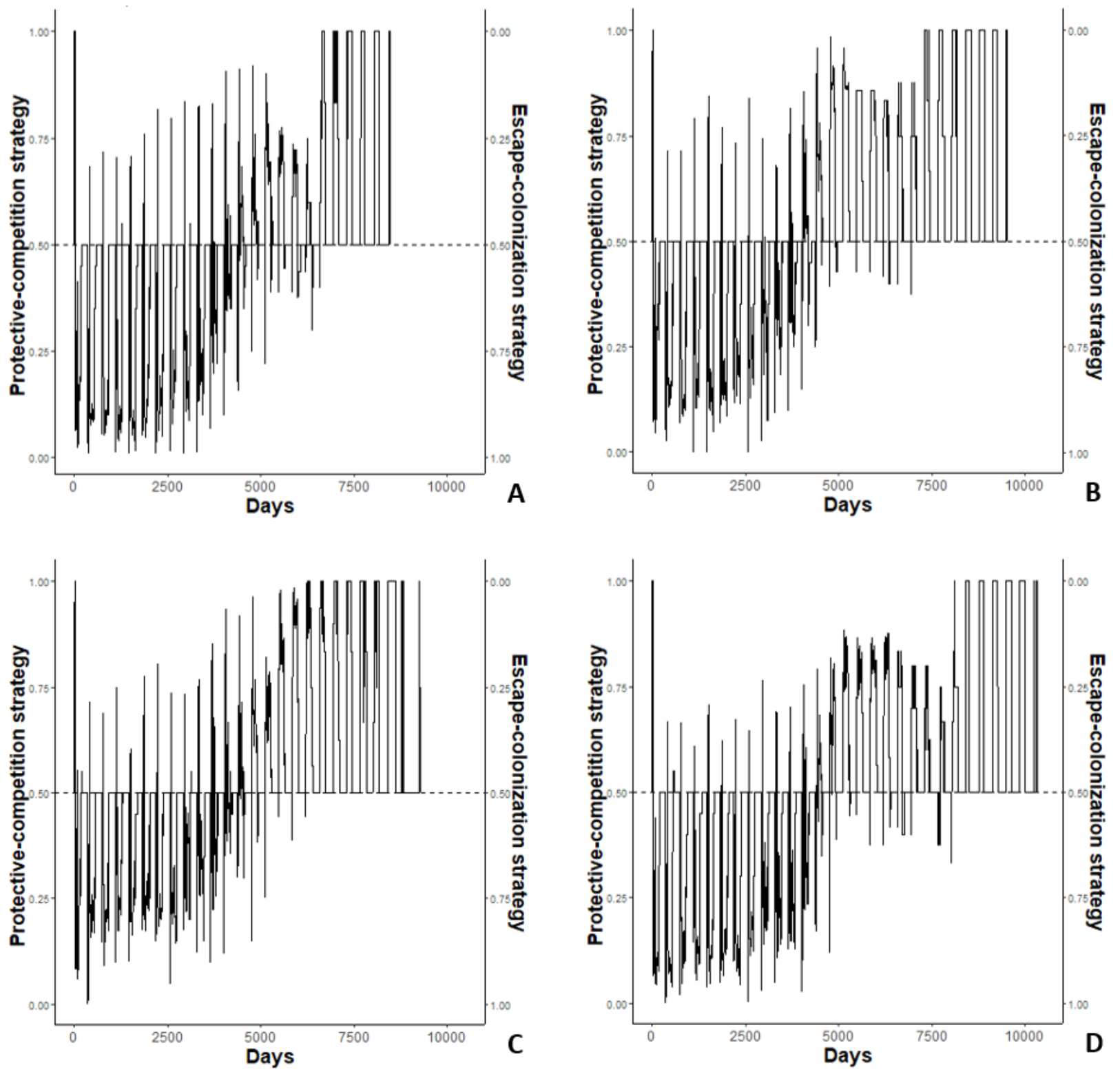
Figure A3.2. Results of simulation runs for the species with the protective-competition strategy (black) and with the escapecolonization strategy (grey) over simulated time in four scenarios (A: low temporal and spatial heterogeneity, B: low temporal heterogeneity and high spatial heterogeneity, C: high temporal heterogeneity and low spatial heterogeneity, D: high temporal and spatial heterogeneity).

5) Proportion of the number of seeds
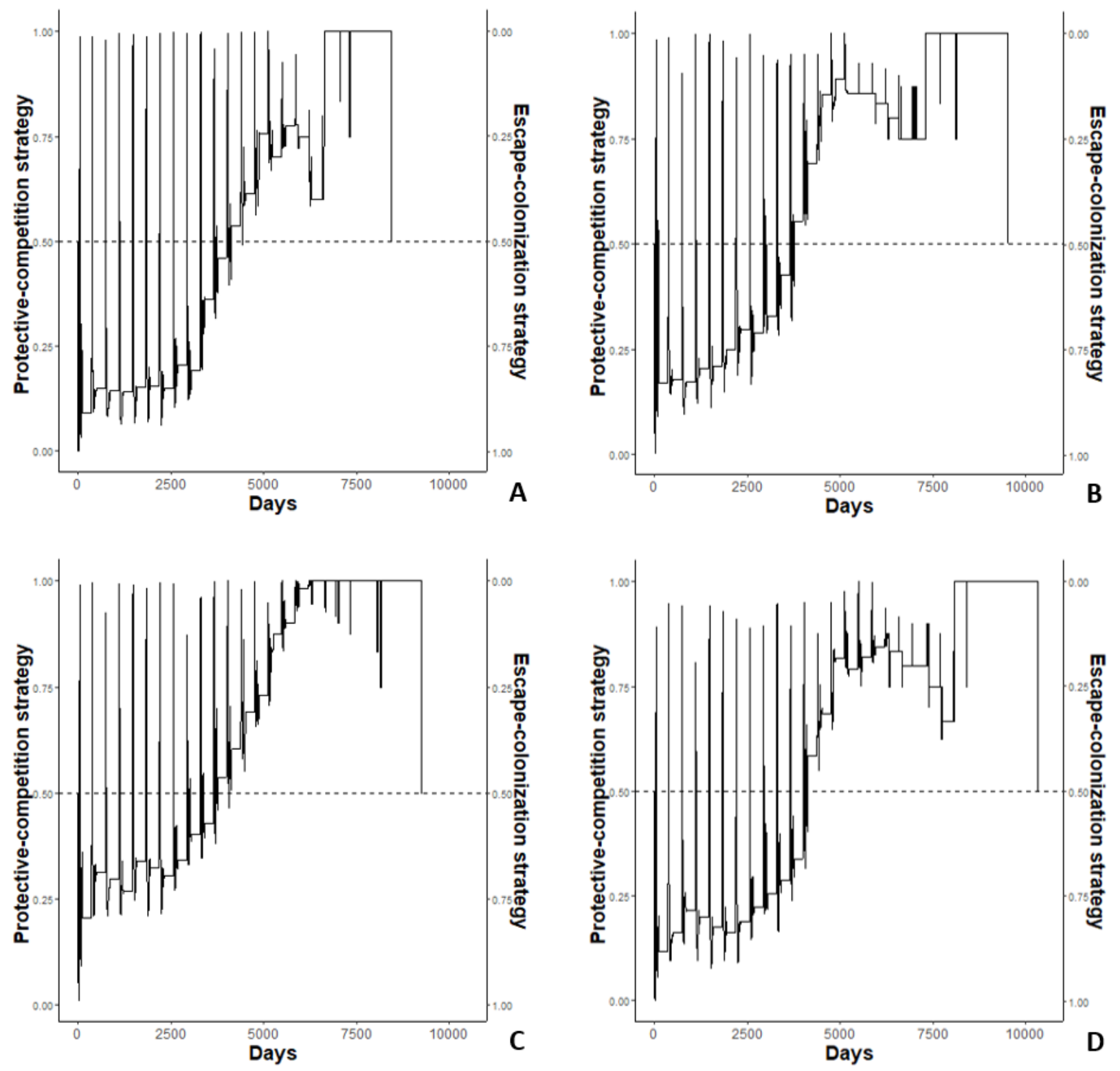


\section{Appendix. Chapter 4.}

Table A4.1. Output of the summary()-function in R applied to the GLMs as described in the main text for six plant functional traits as response variables: dry weight, number of leaves, seed germination probability, osmolyte concentration, stomatal conductance, and photosynthetic rate. EC: soil electrical conductivity of the site of origin; AI: De Martonne aridity index, SR: species richness, HS: treatment with adding salt solution. EC x AI, EC x HS, AI x HS, SR x HS, and EC x AI x HS are interactions between the corresponding variables.

\begin{tabular}{|c|c|c|c|c|c|c|c|c|c|}
\hline \multicolumn{5}{|c|}{ Dry weight } & \multicolumn{5}{|c|}{ Osmolyte concentration } \\
\hline & Estimate & Error & $\mathrm{t}$ value & $\operatorname{Pr}(>|t|)$ & & Estimate & Std. Error & $\mathrm{t}$ value & $\operatorname{Pr}(>|t|)$ \\
\hline Intercept & 0.4038 & 1.5796 & 0.2556 & 0.7989 & Intercept & 0.0013 & 0.0006 & 2.1555 & 0.0369 \\
\hline $\mathrm{EC}$ & 0.0007 & 0.0005 & 1.3237 & 0.1897 & $\mathrm{EC}$ & $-6.6 \mathrm{E}-08$ & $2.0 \mathrm{E}-07$ & -0.3301 & 0.7430 \\
\hline AI & 4.5892 & 2.2907 & 2.0034 & 0.0488 & $\mathrm{AI}$ & 0.0008 & 0.0009 & 0.9387 & 0.3533 \\
\hline SR & -0.0092 & 0.0087 & -1.0604 & 0.2924 & SR & $6.4 \mathrm{E}-07$ & $3.6 \mathrm{E}-06$ & 0.1806 & 0.8576 \\
\hline HS & -1.5176 & 2.1477 & -0.7066 & 0.4820 & $\mathrm{HS}$ & -0.0010 & 0.0006 & -1.5347 & 0.1324 \\
\hline $\mathrm{EC} \times \mathrm{AI}$ & -0.0009 & 0.0005 & -1.8430 & 0.0693 & $\mathrm{EC} \times \mathrm{AI}$ & -1.17 E-08 & $1.9 \mathrm{E}-07$ & -0.0602 & 0.9523 \\
\hline EC x HS & 0.00001 & 0.0007 & 0.0134 & 0.9893 & EC x HS & $9.8 \mathrm{E}-08$ & $2.09 \mathrm{E}-07$ & 0.4708 & 0.6402 \\
\hline AI x HS & 0.2910 & 3.1078 & 0.0936 & 0.9256 & AI x HS & -0.0006 & 0.0009 & -0.6095 & 0.5455 \\
\hline $\begin{array}{l}\text { SR } x \text { HS } \\
\text { EC x AI x }\end{array}$ & 0.0145 & 0.0116 & 1.2496 & 0.2154 & $\begin{array}{l}\text { SR } x \text { HS } \\
\text { EC } \times \text { AI } x\end{array}$ & -1.7 E-06 & 3.7 E-06 & -0.4633 & 0.6456 \\
\hline HS & 0.0000 & 0.0006 & -0.0431 & 0.9657 & HS & $-2.9 \mathrm{E}-08$ & $2.04 \mathrm{E}-07$ & -0.1431 & 0.8869 \\
\hline \multicolumn{5}{|c|}{$\begin{array}{c}\text { Number of leaves } \\
\text { Std. }\end{array}$} & \multicolumn{5}{|c|}{ Stomatal conductance $\mathbf{g}_{\mathrm{s}}$} \\
\hline & Estimate & Error & $\mathrm{z}$ value & $\operatorname{Pr}(>|z|)$ & & Estimate & Std. Error & $\mathrm{t}$ value & $\operatorname{Pr}(>|t|)$ \\
\hline Intercept & 4.1526 & 0.1750 & 23.7325 & $1.67 \mathrm{E}-124$ & Intercept & -19.3232 & 8.1241 & -2.3785 & 0.0212 \\
\hline $\mathrm{EC}$ & 0.00002 & 0.0001 & 0.3222 & 0.7473 & $\mathrm{EC}$ & 0.0049 & 0.0032 & 1.5162 & 0.1358 \\
\hline AI & 1.4387 & 0.2504 & 5.7460 & 0.0000 & $\mathrm{AI}$ & 40.4050 & 12.2174 & 3.3072 & 0.0018 \\
\hline SR & -0.0020 & 0.0009 & -2.0879 & 0.0368 & SR & -0.0316 & 0.0572 & -0.5516 & 0.5837 \\
\hline HS & -1.1353 & 0.2977 & -3.8141 & 0.0001 & $\mathrm{HS}$ & 7.9093 & 12.5921 & 0.6281 & 0.5328 \\
\hline $\mathrm{EC} \times \mathrm{AI}$ & -0.0001 & 0.0001 & -2.4696 & 0.0135 & $\mathrm{EC} \times \mathrm{AI}$ & -0.0070 & 0.0029 & -2.3789 & 0.0212 \\
\hline EC $x$ HS & 0.0002 & 0.0001 & 1.9458 & 0.0517 & EC x HS & 0.0002 & 0.0047 & 0.0418 & 0.9669 \\
\hline AI x HS & 0.2277 & 0.4272 & 0.5330 & 0.5940 & AI x HS & -13.3839 & 18.3103 & -0.7309 & 0.4682 \\
\hline $\begin{array}{l}\text { SR } x \text { HS } \\
\text { EC } \times A I x\end{array}$ & 0.0030 & 0.0015 & 1.9395 & 0.0524 & $\begin{array}{l}\text { SR } \times \text { HS } \\
\mathrm{EC} \times \mathrm{AI} \times\end{array}$ & 0.0395 & 0.0853 & 0.4632 & 0.6452 \\
\hline & -0.0001 & 0.0001 & -1.6343 & 0.1022 & & 0.0011 & 0.0044 & 0.2567 & 0.7985 \\
\hline
\end{tabular}

\begin{tabular}{|c|c|c|c|c|c|c|c|c|c|}
\hline & \multicolumn{3}{|c|}{$\begin{array}{c}\text { Seed germination probability } \\
\text { Std. }\end{array}$} & \multirow[b]{2}{*}{$\operatorname{Pr}(>|z|)$} & \multicolumn{5}{|c|}{ Photosynthetic rate $A_{N}$} \\
\hline & Estimate & Error & $\mathrm{z}$ value & & & Estimate & Std. Error & $\mathrm{t}$ value & $\operatorname{Pr}(>|t|)$ \\
\hline Intercept & 5.9828 & 1.6054 & 3.7267 & 0.0002 & Intercept & -0.2177 & 0.0983 & -2.2144 & 0.0314 \\
\hline $\mathrm{EC}$ & -0.0012 & 0.0005 & -2.6173 & 0.0089 & $\mathrm{EC}$ & 0.00002 & 0.00004 & 0.5209 & 0.6047 \\
\hline AI & -6.6031 & 2.3144 & -2.8530 & 0.0043 & AI & 0.4974 & 0.1565 & 3.1779 & 0.0025 \\
\hline SR & -0.0022 & 0.0080 & -0.2778 & 0.7812 & SR & -0.0005 & 0.0007 & -0.7390 & 0.4633 \\
\hline HS & -1.9451 & 2.4123 & -0.8064 & 0.4200 & $\mathrm{HS}$ & 0.1340 & 0.1503 & 0.8912 & 0.3771 \\
\hline $\mathrm{EC} \times \mathrm{AI}$ & 0.0014 & 0.0005 & 3.0919 & 0.0020 & EC x AI & -0.0001 & 0.00003 & -1.6080 & 0.1141 \\
\hline EC $x$ HS & 0.0013 & 0.0007 & 1.8282 & 0.0675 & EC x HS & 0.00003 & 0.0001 & 0.4975 & 0.6210 \\
\hline AI x HS & 3.0932 & 3.4505 & 0.8965 & 0.3700 & AI x HS & -0.2167 & 0.2297 & -0.9432 & 0.3501 \\
\hline SR x HS & -0.0042 & 0.0124 & -0.3380 & 0.7354 & SR x HS & -0.0002 & 0.0010 & -0.2150 & 0.8306 \\
\hline $\begin{array}{l}\text { EC x AI x } \\
\text { HS }\end{array}$ & -0.0012 & 0.0007 & -1.6671 & 0.0955 & $\begin{array}{l}\text { EC x AI x } \\
\text { HS }\end{array}$ & -0.000007 & 0.0001 & -0.1221 & 0.9033 \\
\hline
\end{tabular}




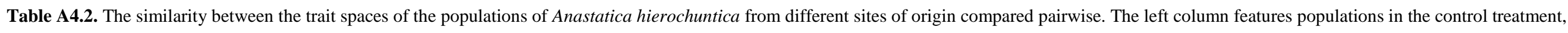

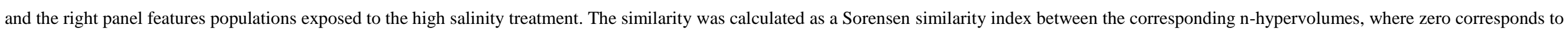

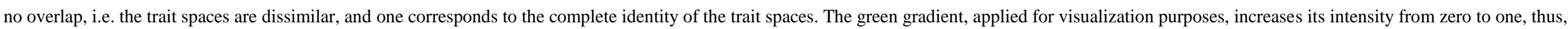

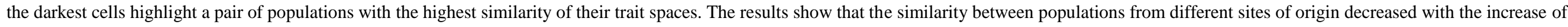

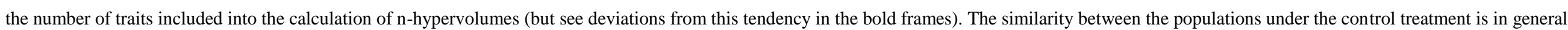

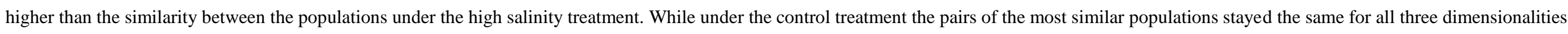

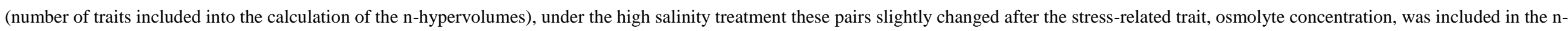
hypervolume calculations (right column, 5 traits).

\begin{tabular}{|c|c|c|c|c|c|c|c|c|c|c|c|}
\hline \multicolumn{6}{|c|}{ Control } & \multicolumn{6}{|c|}{ High salinity } \\
\hline 3 traits & Meishar & Uvda & Timna & Shaharut & Shefech Zohar & 3 traits & Meishar & Uvda & Timna & Shaharut & Shefech Zohar \\
\hline Meishar & --- & 0.4230088 & 0.4958752 & 0.51466793 & 0.5049467 & Meishar & --- & 0.3545028 & 0.280438 & 0.3973088 & 0.3991937 \\
\hline Uvda & 0.4230088 & --- & 0.5327947 & 0.6251874 & 0.4645672 & Uvda & 0.3545028 & --- & 0.2552211 & 0.3088084 & 0.4097245 \\
\hline Timna & 0.4958752 & 0.5327947 & --- & 0.499107 & 0.6369013 & Timna & 0.280438 & 0.2552211 & --- & 0.53964 & 0.32688318 \\
\hline Shaharut & 0.51466793 & 0.6251874 & 0.499107 & --- & 0.5017108 & Shaharut & 0.3973088 & 0.3088084 & 0.53964 & --- & 0.3654795 \\
\hline Shefech Zohar & 0.5049467 & 0.4645672 & 0.6369013 & 0.5017108 & --- & Shefech Zohar & 0.3991937 & 0.4097245 & 0.32688318 & 0.3654795 & --- \\
\hline
\end{tabular}

4 traits

Uvda

Timna

Shaharut

Shefech Zohar

\begin{tabular}{|l|c|c|r|r|}
\hline-- & 0.3505871 & 0.2973164 & 0.4727335 & 0.3304611 \\
\hline 0.3505871 & --- & 0.2105008 & 0.5013843 & 0.2049913 \\
\hline 0.2973164 & 0.2105008 & --- & 0.3305808 & 0.6291125 \\
\hline 0.4727335 & 0.5013843 & 0.3305808 & -- & 0.352409 \\
\hline 0.3304611 & 0.2049913 & 0.6291125 & 0.352409 & --- \\
\hline
\end{tabular}

4 traits

\begin{tabular}{|c|c|c|c|c|c|}
\hline Meishar & --- & 0.2274975 & 0.16441015 & 0.2212537 & 0.3631246 \\
\hline Uvda & 0.2274975 & --- & 0.14218779 & 0.09197658 & 0.3963042 \\
\hline Timna & 0.16441015 & 0.14218779 & --- & 0.2532454 & 0.14505188 \\
\hline Shaharut & 0.2212537 & 0.09197658 & 0.2532454 & --- & 0.0898632 \\
\hline Shefech Zohar & 0.3631246 & 0.3963042 & 0.14505188 & 0.0898632 & --- \\
\hline
\end{tabular}

\begin{tabular}{|c|c|c|c|c|c|c|c|c|c|c|c|}
\hline 5 traits & & & & & & 5 traits & & & & & \\
\hline Meishar & --- & 0.3828005 & 0.12177464 & 0.4321779 & 0.11445989 & Meishar & --- & 0.1585687 & 0.02568206 & 0.03979347 & 0.1069296 \\
\hline Uvda & 0.3828005 & --- & 0.13760159 & 0.3710848 & 0.07071976 & Uvda & 0.1585687 & --- & 0.014436746 & 0.010240631 & 0.02459421 \\
\hline Timna & 0.12177464 & 0.13760159 & --- & 0.1987431 & 0.5048132 & Timna & 0.02568206 & 0.014436746 & --- & 0.09527571 & 0.14708405 \\
\hline Shaharut & 0.4321779 & 0.3710848 & 0.1987431 & --- & 0.12819992 & Shaharut & 0.03979347 & 0.010240631 & 0.09527571 & --- & 0.17476982 \\
\hline Shefech Zohar & 0.11445989 & 0.07071976 & 0.5048132 & 0.12819992 & --- & Shefech Zohar & 0.1069296 & 0.02459421 & 0.14708405 & 0.17476982 & --- \\
\hline
\end{tabular}




\section{Acknowledgements}

Many people have accompanied and supported my PhD journey, and it is very hard not to miss anyone. That is why I would like to start with apologies if I miss someone in the list below. I hope that I said "thank you" in person.

First, none of all these bright and challenging moments on the way to the complete thesis would be possible without Dr. Katrin Meyer who had a brilliant idea for a project some years ago, a project with a $\mathrm{PhD}$ position that I found extremely attractive and interesting to apply for and go to Göttingen for postgraduate studies. I should say that Katrin is the person I have learned the most from, not only because of daily supervision but also because of her openness to provide feedback timely and constructively, and her ability to be a great role model for a young scientist. It is impossible to express the amount of professional and personal help I have received from her through these (almost) four years. I just can say that Katrin was there for me every time I needed some guidance or felt a bit lost in my $\mathrm{PhD}$ studies and always lent a hand to set priorities and shape the next goals. I am still fascinated by her ability to read my very first drafts and find their bright sides and encourage me to continuously improve them.

I am also thankful to my PhD Committee members Prof. Kerstin Wiegand and Prof. Holger Kreft for regular meetings and feedback on my $\mathrm{PhD}$ progress, which were beyond the formal reports and delivered me the bigger picture, standards and scientific frameworks on my project. The role of Prof. Kerstin Wiegand for my PhD was not limited to these regular meetings. I am grateful for the opportunities to ask some short but urgent questions and for a chance to stay in the group until I am done with my thesis. Certainly, an open and encouraging atmosphere of the entire research group is Kerstin's merit. I value the group seminars that bring us together and allow not only regularly presenting and evaluating my work but also discovering new points of view and perspectives. These exchanges would not be possible without my group fellows who were constantly available for short friendly consultations on technical issues or very thorough checks of my model codes. Thank you, Sebastian Hanss, Dr. Jan Salecker, Dr. Maximilian Hesselbarth and all others. In addition, I should not underestimate the importance of small chats here and there for a fruitful research atmosphere. Another big 
thank you goes to Ilona Watteler-Spang and Dörte Dede for saving me from the mysterious world of paperwork related to the university and my research.

For insightful intense scientific discussions, I am very grateful to our project partners in Israel, primarily to Prof. Merav Seifan from Sde Boker, who found time for me despite having to deal with piles of her own work. It was a lot of fun to shape our manuscripts in constant discussions and exchange of opinions and ideas. Furthermore, the opportunity to cooperate with Merav's group, to visit the research site and live the life of a field ecologist for a while was an important contribution to my understanding of the project and the desert. Another exciting project arose from a manuscript on metamodels driven by $\mathrm{PhD}$ students and led by Bruno Pietzsch, TU Dresden. Thanks to it, I got a taste of such peer-to-peer cooperation.

Although I do not know who they were, I am grateful for the just and tactful reviewers of my first first-author manuscript. Despite the necessity for a major revision, it was a pleasant experience and encouraged me to keep working and writing.

Our small support groups of writing together and organizing our weekly and monthly research routine became an important contribution to my wellbeing and health throughout the toughest times of my $\mathrm{PhD}$. We were together in different but so similar challenging situations, and, I hope, will all soon successfully finish our $\mathrm{PhD}$ journeys.

Last but not the least, I am thankful to my family back in Russia for their understanding of my career moves and patience while waiting for my rare visits. I am also grateful to my in-laws in Israel, who made my work visits even more enjoyable. I cannot but say thank you to my freshly established family here in Germany, to my husband Peleg, who supported and inspired me starting from application for a $\mathrm{PhD}$ program and throughout it.

The project underlying this thesis has become possible thanks to "Niedersächsisches Vorab" (MWK ZN3155) and Volkswagen Foundation. 


\section{Curriculum vitae}

PERSONAL

INFORMATION

$10 / 14-09 / 16$

$03 / 16$

$10 / 08-02 / 12$

$09 / 06-06 / 08$

$09 / 02-06 / 06$

\section{Liubov Zakharova}

Address: Zimmermannstr. 19, 37075, Germany

Date of birth: 04/01/1985

Place of birth: Woroschilowgrad, Ukraine, Nationality: Russian

E-mails: liubov.zakharova@uni-goettingen.de

$\mathrm{PhD}$ program - Forest Science and Ecology

University of Göttingen, Department of Ecosystem Modelling, Germany

- Specialization: Ecological Modelling

- PhD thesis topic: Modelling of population- and community-level plant trait variability in changing arid environments

11th Summer School in Individual- and Agent-Based Modeling

Technische Universität Dresden, Germany

- Analysis and application of individual- and agent-based modelling

Master of Science - Biodiversity, Evolution and Ecology (grade 1.3)

Freie Universität Berlin, Germany

- Specialization: Ecological Modelling

- Thesis: Using ecohydrological plant traits to model and predict the impact of altered precipitation patterns on the performance of individual plants in warm drylands

(R language)

Winter School for Ecological Modelling

Helmholtz Centre for Environmental Research - UFZ, Leipzig, Germany

- Introduction to $\mathrm{C}+$, theory of ecological modelling

Diploma - Management (the grade equates to 1.0)

Institute for Management, Marketing and Finance, Voronezh, Russia

- Specialization: Organization management

- Thesis: Image building of the organization (for the Interregional Human Rights Group)

Master of Science - Biology (the grade equates to 1.0)

Voronezh State University, Russia

- Specialization: Ecology

- Thesis: The consortive analysis of the genus Crataegus L. (Rosaceae Juss.) in the city Voronezh

Bachelor of Science - Biology (the grade equates to 1.0)

Voronezh State University, Russia

- Specialization: Biology and Ecology of Plants

- Thesis: Representatives of the genus Crataegus L. (Hawthorns) in the Botanical garden of the Voronezh State university 
PUBLICATIONS

\section{WORK EXPERIENCE \\ since 2015}

$07 / 20$ - now

$10 / 16-06 / 20$

$04 / 16-09 / 16$

$10 / 15-03 / 16$
- The International Society for Ecological Modelling. Global conference, October 2019, Presentation "Individual- and trait-based models as a tool for revealing plant community drivers in deserts"

- Workshop Young Modellers in ecology, May 2019, Presentation "Modelling plant communities in a desert under water and data scarcity"

- Conference Gesellschaft für Ökologie, September 2018, Presentation "Using a trait-based model to study plant strategies under severe desert conditions"

- Workshop Young Modellers in ecology, May 2018, Poster "How to survive hard times and prosper in good times? Trait-based modelling as a tool for understanding plant community dynamics in a desert"

- Conference British ecology society/ Gesellschaft für Ökologie, December 2017, Poster "Trait-based modelling as a tool for understanding community dynamics"

- Scientific Cooperation between Israel and Lower Saxony - Spring Meeting, March 2017, Poster "Combining empirical and modelling approaches to assess plant trait variability at different salinity and water availability levels"

- Israeli Plant ecology conference, January 2017, Poster "Modelling plant trait variability in changing arid environments as part of an Israeli-German empirical-modelling project"

- Conference Gesellschaft für Ökologie, September 2016, Poster "Modelling plant hydraulic strategies in drylands"

- Zakharova, L., Meyer, K. M., \& Seifan, M. (2020). Combining trait-and individual-based modelling to understand desert plant community dynamics. Ecological Modelling, 434, 109260.

- $\quad$ Pietzsch, B., Fiedler, S., Mertens, K.G., Richter, M., Scherer, C., Widyastuti, K., Wimmler, M.-C., Zakharova, L., Berger, U. (2020). Metamodels for Evaluating, Calibrating and Applying Agent-Based Models: A Review. JASSS, 23 (2) 9.

- Zakharova, L., Meyer, K. M., \& Seifan, M. (2019). Trait-based modelling in ecology: A review of two decades of research. Ecological Modelling, 407, 108703.

- $\quad$ Ratzmann, G., Zakharova, L., \& Tietjen, B. (2019). Optimal leaf water status regulation of plants in drylands. Scientific reports, 9(1), 3768.

\section{Research Associate}

Ecological Modelling, ibacon GmbH, Germany

Scientific Assistant

Department of Ecosystem Modelling, Büsgen-Institute, University of Göttingen, Germany

Student Assistant

Functional Biodiversity, Freie Universität Berlin, Germany

Tutor for Biochemistry

Freie Universität Berlin, Germany 